\title{
MASSIVE MEDIA: \\ THEORIES AND PRACTICES OF LARGE-SCALE PROJECTIONS AND PUBLIC DATA VISUALIZATIONS
}

\author{
by David Robert Colangelo \\ Master of Cultural Studies, Goldsmiths College, London, England, 2007 \\ Bachelor of Arts \& Science, McMaster University, Hamilton, Ontario, Canada, 2006
}

\author{
A dissertation \\ Presented to Ryerson University and York University \\ in partial fulfillment of the \\ requirements for the degree of \\ Doctor of Philosophy \\ in the Program of \\ Communication and Culture
}

Toronto, Ontario, Canada, 2015

(C) Dave Colangelo 2015 


\section{Author's Declaration For Electronic Submission Of A Dissertation}

I hereby declare that I am the sole author of this dissertation. This is a true copy of the dissertation, including any required final revisions, as accepted by my examiners.

I authorize Ryerson University to lend this dissertation to other institutions or individuals for the purpose of scholarly research.

I further authorize Ryerson University to reproduce this dissertation by photocopying or by other means, in total or in part, at the request of other institutions or individuals for the purpose of scholarly research.

I understand that my dissertation may be made electronically available to the public. 


\author{
Abstract \\ Massive Media: Theories and Practices of \\ Large-Scale Projections and Public Data Visualizations \\ Doctor of Philosophy, 2015 \\ David Robert Colangelo \\ Communication and Culture \\ Ryerson University and York University
}

This dissertation describes, historicizes, theorizes, and deploys "massive media," an emerging subset of technical assemblages that include large outdoor projections, programmable architectural façades, and urban screens. Massive media are massive in their size and subsequent visibility, but are also an agglomeration of media in their expressive screen and cinema-like qualities and their associated audio, interactive, and network capabilities. This dissertation finds that massive media enable and necessitate the development of new practices of expanded cinema, public data visualization, and new media art and curation that blend the logics of urban space, monumentality, and the public sphere with the aesthetics and affordances of digital information and the moving image to support a more participatory public culture in which we identify and engage with collective presence, memory, and action through information, architecture, and the moving image. Through historical research, case studies, conversations with cultural producers, participant observation, and creation-as-research projects, large-scale public projections are shown to represent a new monumentality that can be better understood and evaluated using analytical tools from cinema studies, namely superimposition, montage, and apparatus/dispositif. Low-resolution LED façades, while sharing some of the 
functional and theoretical characteristics of projection, are shown to uniquely support an emerging practice of public data visualization and represent a more consistent embodiment of a hybrid and relational public sphere through a tighter coupling of information, architecture, and context. Programmable architectural façades, more than projections, embody the development of supermodernism in architecture where data-rich public spaces of identity, congregation, and contestation seek and find appropriate and consistent outlets in highly visible spatial assemblages of architecture and media. Finally, a curatorial approach to massive media is crucial in order to create suitable spaces and opportunities for the development of massive media as a legitimate art form. This requires the sustained provision of technical support and coordination as well as an ongoing negotiation with corporate, institutional, and civic owners and operators. While massive media exists primarily as a highly commercialized phenomenon, it can also be pressed into service, through coordinated curatorial and artistic efforts, to critique or co-opt commercialization, and to re-envision the role of urban media environments in shaping collective identity, historical consciousness, and public display culture. 


\section{Acknowledgements}

If I had to identify a starting point for this dissertation it would be the moment the organizers of Nuit Blanche in Toronto selected my proposal to use the LED façade of the CN Tower as a collective, interactive beacon for the city's 'energy.' My interest in massive media was in its nascent phase at the time and may have quickly faded had they not placed their faith in me. It was around that same time that I met Patricio Davila. Over the past five years we have collaborated on number of massive media projects, testing and refining our theories and techniques with this medium along the way. Nothing has been more productive to my thinking on this subject than the work we have done together and the conversations we have had in his champagne-coloured Volvo station wagon.

Early on in the process of conceiving of, researching, and writing this dissertation I also connected with Janine Marchessault. She is the kind of academic I aspire to be: actively engaged in fostering and contributing to a community of critical thinkers and doers, locally and globally, through her writing, creating, curating, and teaching. I continue to draw inspiration from her tireless efforts. The support of Paul Moore, Graduate Program Director of Communication and Culture, has been invaluable in this process as well. The genuine care and concern he has shown both towards me and the program has gone a long way in keeping everything and everyone moving in the right direction. Jennifer Fisher provided much needed prompts to think deeper and write better and, along with her Journal of Curatorial Studies co-editor Jim Drobnick, helped to sharpen the chapter on curation. Jo Ann Mackie, Graduate Program Administrator, helped to make this process an enjoyable one. 
Finally I would be remiss if I did not acknowledge the love and support of my family and friends: my grandparents, who were selfless in their sacrifices; Luigi and Antonietta Colangelo, whose care as parents is apparent each and every day; and finally, my brothers Steven and Adam Colangelo and my best friends Dr. Massimo Di Ciano, Mike Moretti, and Jamie Webster, who have been there with me in my battles with big, flashy buildings, and other things too. I could not have done this without all of you, and would not have wanted to anyway. 


\section{Table of Contents}

\section{CHAPTER 1:}

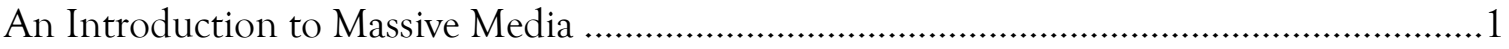

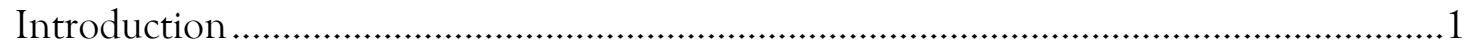

Rationale for Studying Massive Media .......................................................................6

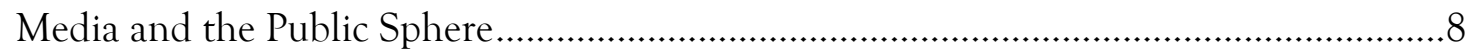

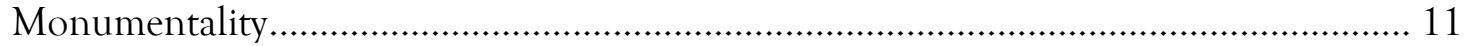

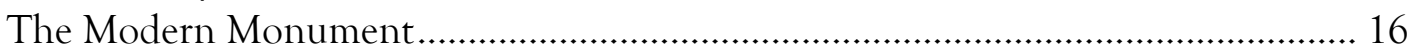

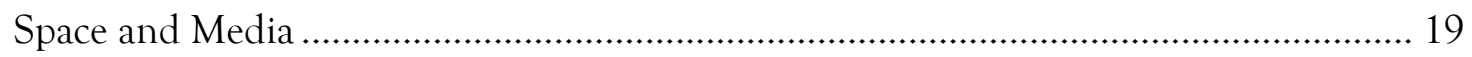

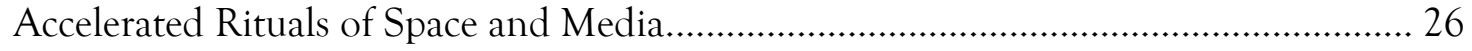

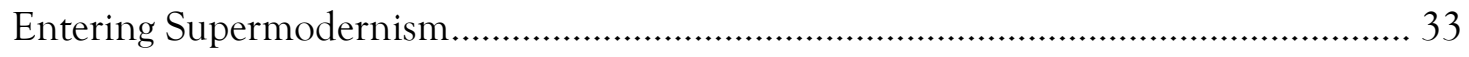

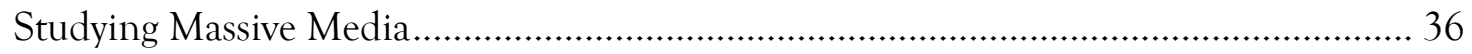

\section{CHAPTER 2:}

Experiments in Large-scale Projection and the (New) New Monumentality ....................... 44

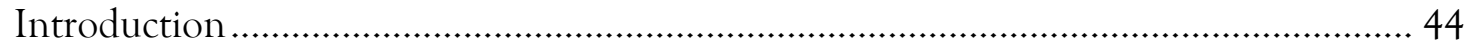

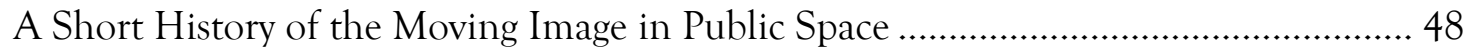

Architecture, Expanded Cinema, and the New Monumentality ....................................56

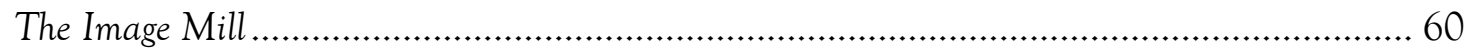

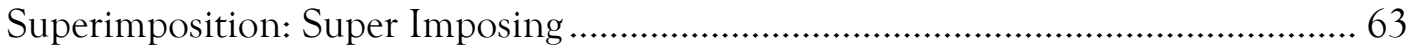

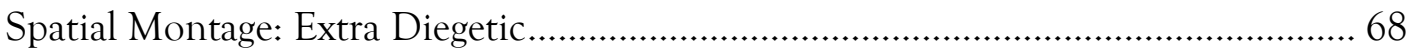

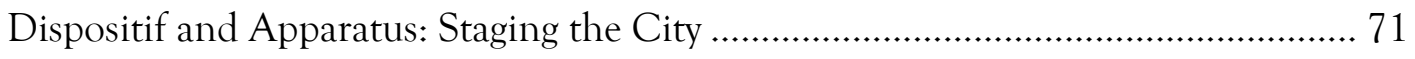

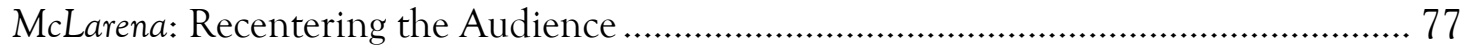

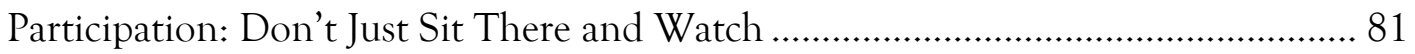

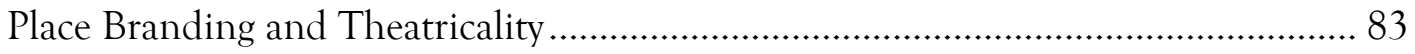

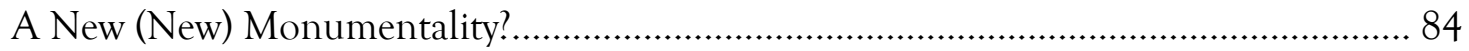

Creation-as-Research: Experiments in Public Projection ............................................ 92

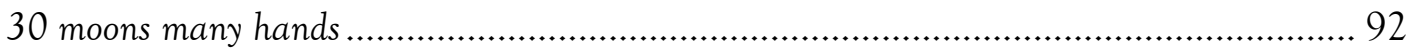

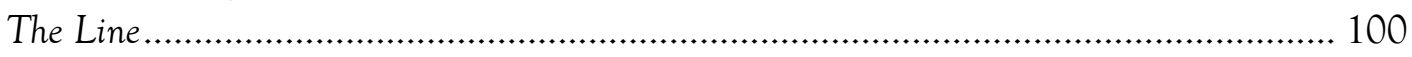

Conclusion: A Perceptual Laboratory for Popular Needs and Aspirations ................... 107

\section{CHAPTER 3:}

The Empire State Building and the Roles of

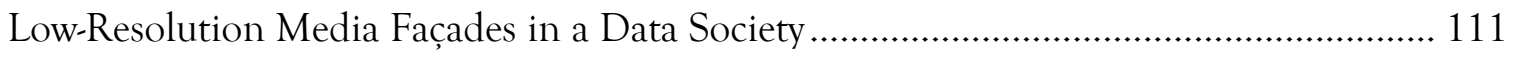

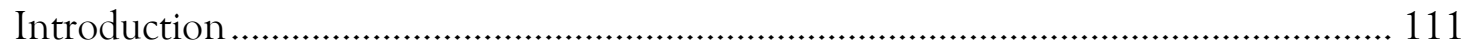

A Short History of The Empire State Building .......................................................... 116

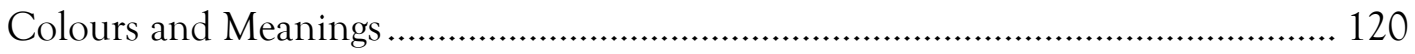

Understanding Contemporary Dimensions of Public Data Visualizations .................. 125

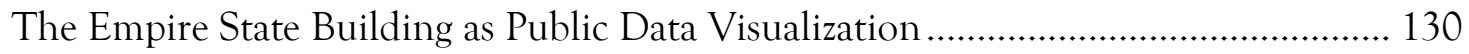

Creation-As-Research: Socially-Engaged Public Data Visualization ............................. 143

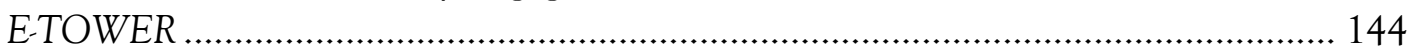

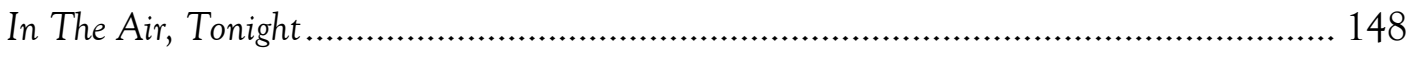

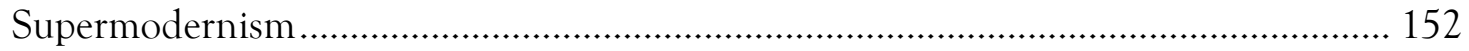


Conclusion: Temporary Intensities and Collective

Conversations in Relational Space....

\section{CHAPTER 4:}

Curating Massive Media 157

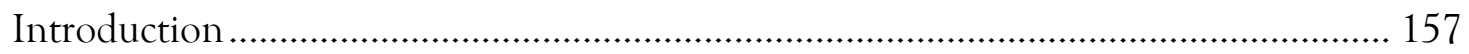

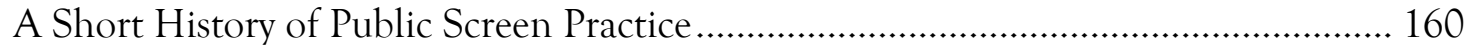

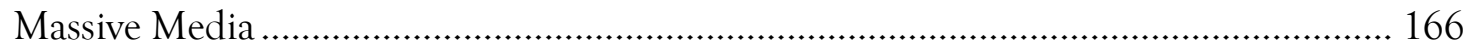

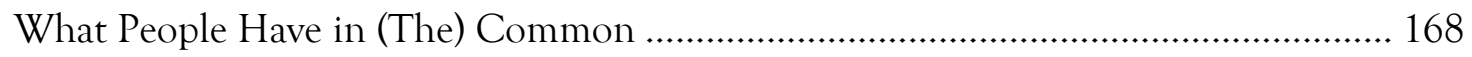

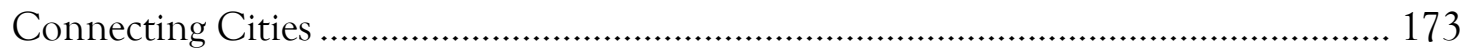

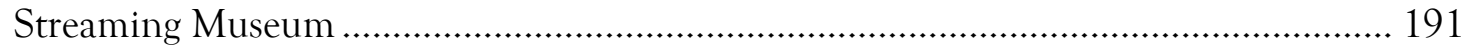

Creation-as-Research: Curating the Ryerson Image Arts Building ............................... 201

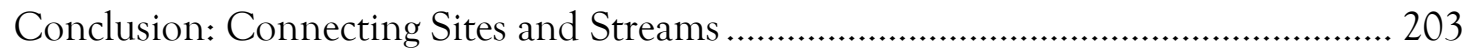

CHAPTER 5:

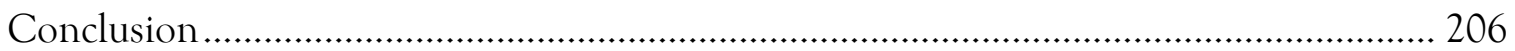

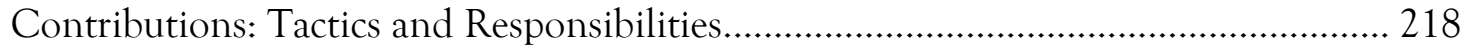

Recommendations and Future Directions: More Massive, More Media...................... 221

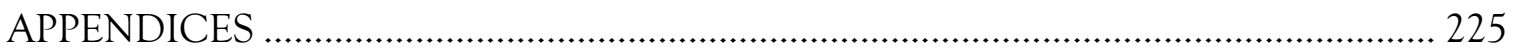

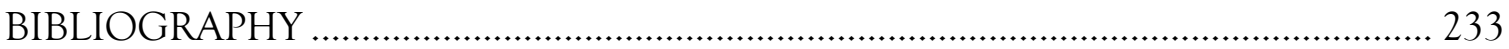




\section{List of Figures}

Figure 1: The CN Tower, Toronto. Photo: CN Tower (C) ................................................. 1

Figure 2. Robert Lepage / Ex Machina, The Image Mill (2008), with the Chateau Frontenac in the foreground, Quebec City. Photo: Christie/Francis Vachon. ..................... 44

Figure 3. Robert Lepage / Ex Machina, The Image Mill (2008), from the lower town with

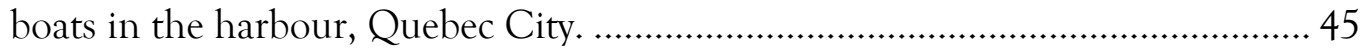

Figure 4. Daily tous les jours, McLarena (2014), Quartiers des spectacles, Montreal. ............. 46

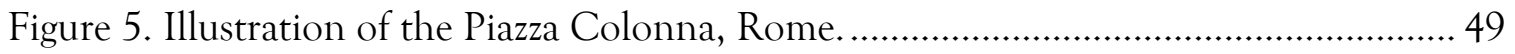

Figure 6. Jenny Holzer, Money Creates Taste (2006), Providence, RI.................................... 53

Figure 7. Krzysztof Wodiczko, Tijuana Projection (2001), Tijuana, Mexico.......................... 54

Figure 8. Charles and Ray Eames, Glimpses of the USA (1959), installation view, Moscow. 56

Figure 9. Portraits of Quebecers from The Image Mill on the façade of the BUNGE, Quebec

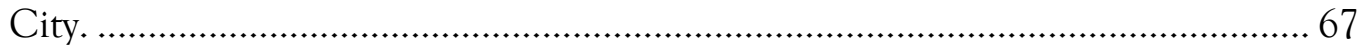

Figure 10. The "Amplified Area" of The Image Mill. Source: lacaserne.net. .......................... 67

Figure 11. Ideal viewing positions for The Image Mill. Source: lacaserne.net. ...................... 72

Figure 12. Viewing area for The Image Mill. Source: UNESCO........................................... 74

Figure 13. Stone walls with stained glass projected at scale. Photo: Nicolas Ruel................ 76

Figure 14. Daily tous les jours, McLarena (2014), Quartiers des spectacles, Montréal............ 78

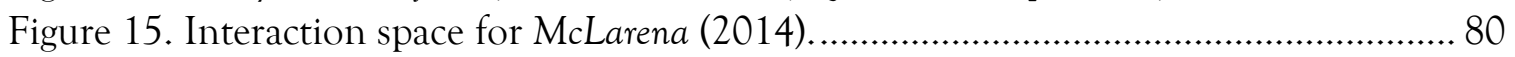

Figure 16. Dave Colangelo and Patricio Davila, 30 moons many hands (2013), installation

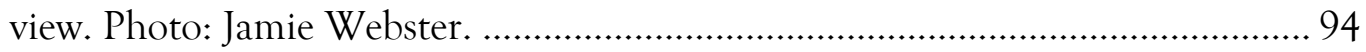

Figure 17. Dave Colangelo and Patricio Davila, 30 moons many hands (2013), installation

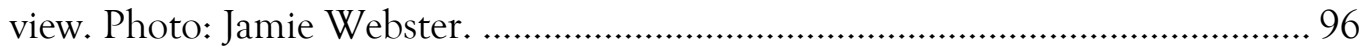

Figure 18. Dave Colangelo and Patricio Davila, 30 moons many hands (2013), installation view. Photo: Jamie Webster. ................................................................................ 97

Figure 19. Dave Colangelo and Patricio Davila, The Line (2013), installation view (snow fence). Photo: Will Pemulis.................................................................................. 101

Figure 20. Dave Colangelo and Patricio Davila, The Line (2013), installation view (projection). Photo: Will Pemulis. ........................................................................... 102

Figure 21. Dave Colangelo and Patricio Davila, The Line (2013), installation view (projection). Photo: Will Pemulis.

Figure 22. Empire State Building, live light show with Alicia Keys, November 26, 2012, YouTube 112

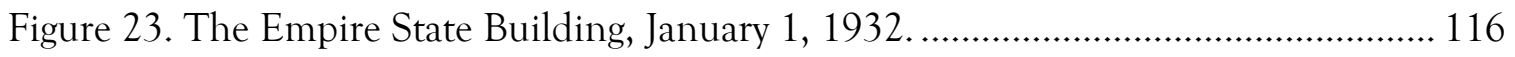

Figure 24. Calendar card for Canada Life Building with weather beacon guide. ............. 118

Figure 25. The Empire State Building's "Freedom Lights" in 1976................................. 120

Figure 26. The Empire State Building with Philips Color Kinetics System. ..................... 122

Figure 27. Empire State Building with CNN election results for Presidential election, 2012.

. 123

Figure 28. Screen capture from 'sgoralnick' during the 2012 election, Instagram............ 124 Figure 29. HeHe (Helen Evans and Heiko Hansen), Pollstream-Nuage Vert (2008), Helsinki.

Figure 30. Onur Sönmez and Tamer Aslan, NOX (2013), Ars Electronica Center, Linz. 129 
Figure 31. Categorized uses of the Empire State Building's lights, 2009-2013.

Figure 32. Verizon's \#WhosGonnaWin promotion for the 2014 Super Bowl.

Figure 33. Empire State Building with blue lights indicating President Obama's victory in

2012 election, '@vanitfair', 2012, Instagram........................................................... 137

Figure 34. Selected Twitter results for "empire state building" during 2012 election....... 138

Figure 35. Nelson Mandela tribute on '@empirestatebldg', 2013, Instagram.................... 139

Figure 36. Dave Colangelo and Patricio Davila, E-TOWER (2010), CN Tower, Toronto.144

Figure 37. Dave Colangelo and Patricio Davila, In The Air, Tonight (2014). Ryerson Image

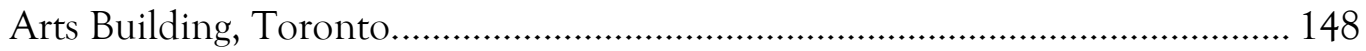

Figure 38. Tracey Emin, I Promise to Love You (2013), Times Square Arts Midnight

Moment, New York. Photo: Ka-Man Tse @TSqArts, courtesy Times Square Arts.

Figure 39. Alfredo Jaar, A Logo for America (1987), commissioned by the Public Art Fund for the Spectacolor Sign, Times Square, New York, April 1987. Photo courtesy of the artist and Galerie Lelong, New York (C) Alfredo Jaar.

Figure 40. starrs \& cmielewski, sms_origins (2009-2010), Federation Square, Melbourne.

Photo: Josephine Starrs and Leon Cmielewski, courtesy of the artists............... 165

Figure 41. Tom Carr, Arrival - Departure (2013), Federation Square and Streaming

Museum, Melbourne. Photo: Greta Jaeger, courtesy Art Plural Gallery..... 168

Figure 42. Richard Wilhelmer, Julius von Bismarck, Benjamin Maus, Mood Gasometer (2008), Media Façades Festival 2008, Berlin Gasometer, Berlin. Photo: courtesy of the artists.

Figure 43. h.o, Connecting Monsters (2013), Connecting Cities, Ars Electronica Center,

Linz, Austria. Photo: Pascal Maresch, courtesy Ars Electronica.......................... 174

Figure 44. Connecting Cities Network. Courtesy Susa Pop.......................................... 178

Figure 45. Varvara Guljajeva and Mar Canet Sola, Binoculars to... Binoculars from... (2013),

Connecting Cities, Bauhaus Dessau. Photo: Ruthe Zuntz, courtesy Public Art Lab.

Figure 46. Frederic Granon, BigBro (2013), \#ProgramaLaPlaza, Medialab-Prado, Madrid.

Photo: courtesy Medialab-Prado........................................................................ 185

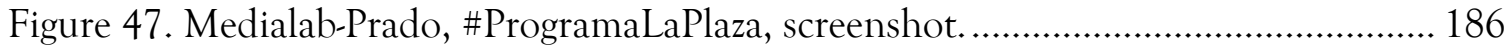

Figure 48. Pfadfinderei + The Constitute, Dancing in the Rain (2013), Connected Cultures

Programme, Galeria de Arte Digital SESI-SP, Avenida Paulista, Sao Paulo, Brazil.

Photo: verve cultural, courtesy Public Art Lab.

Figure 49. Björk, Mutual Core (2012), Times Square Midnight Moment, Streaming

Museum \& MOCAtv, New York. Photo: Ka-Man Tse, courtesy Streaming

Museum.

Figure 50. Streaming Museum Network, 2014. Courtesy Nina Colosi.....

Figure 51. Maurice Benayoun, Emotion Forecast (2012), screenshot. Courtesy Streaming

Museum.

Figure 52. Maurice Benayoun, Occupy Wall Screens (2012), Big Screen Plaza, New York

City. Photo: David Bates, Jr., courtesy Streaming Museum. 


\section{List of Appendices}

APPENDIX 1: Ryerson Image Arts Building Proposal .......................................................225

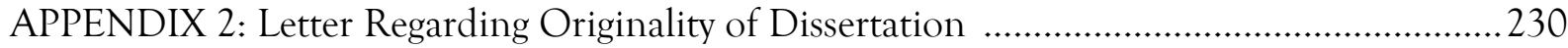

APPENDIX 3: Re: Letter Regarding Originality of Dissertation ..........................................232 
CHAPTER 1: An Introduction to Massive Media

\section{Introduction}

Toronto's CN Tower was built in 1976 as a telecommunications tower. The iconic building rises 553 metres above the city as an omnipresent reference point for anyone within a $20 \mathrm{~km}$ radius. It dominates photos taken by tourists and locals, reflecting the status and character of the city as relatively young and steeped in the brutal modernism of the era in which so much of it was built, simultaneously gesturing towards past, present, and future ambitions.

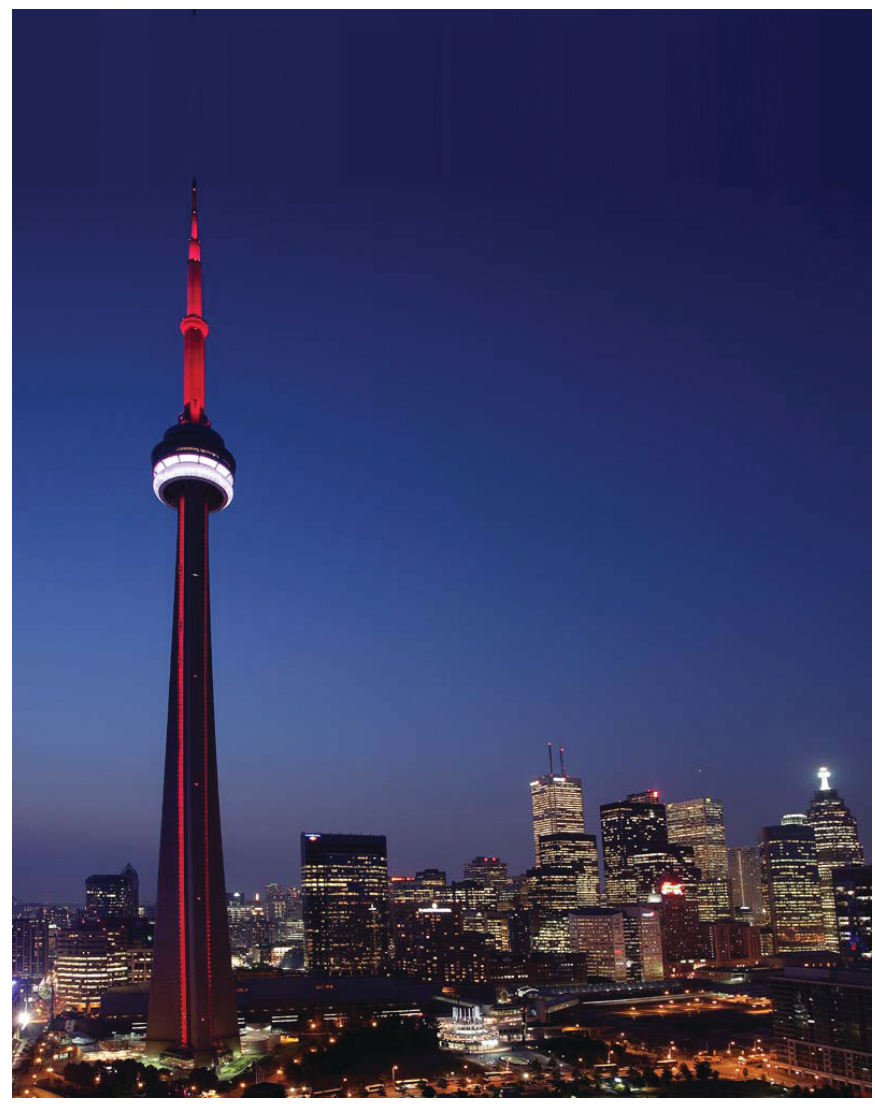

Figure 1: The CN Tower, Toronto. Photo: CN Tower @ 
While the tower has always been prominently lit, it was not until 2007 that the building was fitted with programmable light-emitting diodes, or LEDs. Its seemingly endless elevator shafts, cylindrical observation deck, and sharp antenna became illuminated and animated by a range of changing colours. Certain holidays, events, and causes were celebrated on the building by way of programming the patterns and colours of the lights. Deep runs into the playoffs by local sports teams (sadly, there have been few of these in Toronto) were represented in team colours, the deaths of fallen Canadian soldiers commemorated in patriotic reds and whites, and breast cancer awareness turned the tower bright pink once a year. The $\mathrm{CN}$ Tower website provided a calendar of lighting events to help decode this information, but one might also hear about it on the radio, see it on television, read about it in a newspaper, or come across a social media post depending on who decided to use lights as part of their publicity campaign or chose to identify with the image of the tower. Eventually, the $\mathrm{CN}$ Tower began distributing images of the changing illuminations of the tower on Facebook, Twitter, and Instagram feeds so that people could witness and participate in discussing, liking, and remediating associated images and messages.

The expressive lighting of the CN Tower and its related media and cultural practices mark a significant shift in its role as a building with a monumental presence in urban space, a building of a particular visibility and importance. As the tower became more like a screen, with its animations and colour changes, and as it became more entwined with other media and screens through its remediation on social media, the logic of the monument, as a dense transfer point for civic identity, was merging with the logic of the 
screen, creating an entirely new cultural entity in the process. In the case of the CN Tower, the sober, stoic tower became open to the ephemerality of the digital trace, and became more available, attractive, and open to various causes, concerns, media channels, and conversations. It reflected its place, time, and audience, responding to the city and its celebrations and allowing people to interact with and through its image.

This emerging phenomenon was not limited to Toronto, of course, nor was this the only way light, architecture, and information were being combined in novel ways. Similar to the $\mathrm{CN}$ Tower, the Empire State Building had also adorned its tip with a programmable low-resolution LED façade, promoting it through similar social media channels and even presenting light shows coordinated with internet radio stations, effectively updating the son e lumiere tradition for the digital age. The Empire State Building and the people that live in its vicinity have been uploading videos of these shows to YouTube, effectively extending the viewing area and public sphere inscribed by the tower. In another example, during the 2008 presidential election, the Empire State Building became a massive real-time display for election results, pitting incremental blue and red columns of light against one another on its spire until finally being bathed in blue to signify Barack Obama's victory, all of which was broadcast on CNN. This massive public visualization of data used the history and status of the Empire State Building and the expressivity of its programmable lights to create a spectacular embodiment of data that became the focal point of a worldwide news event. Its digital, screen-like presence performed historical realities in real-time, inserting itself into public discourse in the process. 
In addition to these iconic and monumental displays, critical and creative uses of large public projections and reactive architecture have also changed the nature of what we look up at and interact with in public space. Artists such as Krzysztof Wodiczko and Jenny Holzer have pioneered and developed much of this work, using the power of the monumental building or the pulpit of the public, commercial screen to insert messages of anti-consumerism and criticisms of government policies, exposing the complexities of capital, geopolitics, and identity in powerful, highly visible ways that only massive images in monumental public spaces can provide. Newer works by Raphael Lozano Hemmer, such as his Vectorial Elevation series and Body Movies, extend the possibilities of light and architecture to include the direct participation of people at various sites, as well as incorporating telepresent participation to expand the possibilities for identification and meaning at and between these sites.

Along with low-resolution LED architectural additions that merge physical mass with ephemeral animations, high-resolution large-scale digital projection mappings are now a mainstay of public displays around the world. Synchronized displays across cities, such as Hong Kong's A Symphony of Lights, which incorporated over 40 buildings in the skyline, have become popular for touristic as well as political purposes. Light festivals such as Vivid Sydney and the Fêtes des Lumières in Lyon have transformed entire sectors of cities into digital cinemas and outdoor galleries for public art and spectacle. Coordinated monumental lighting displays have also been incorporated into city-wide protests and demonstrations. In the weeks following the terrorist attacks on the offices of satirical newspaper, Charlie Hebdo, the words "PARIS EST CHARLIE" were projected across the Arc 
De Triomphe and the trademark light show of the Eiffel Tower was dimmed to pay respect to those who had died in the attacks (Keromnes 2015). Both provided powerful symbols of solidarity through light, architecture, and public space with those gathering and demonstrating in the city.

While artistic and political uses of large-scale projections, screens, and media façades multiply in cities, it should come as no surprise that advertisements take up the most space and time on expressive architectural surfaces. Buildings such as the Empire State Building regularly rent their luminous tip to corporations (such as Facebook and Microsoft) or even Broadway shows to display their colours and use the resulting "content" for promotional purposes. For example, a recent promotion for Verizon saw the building display the results of an online poll that asked fans who they thought would win the 2014 Super Bowl in the week leading up to the event, eventually displaying the results of the daily tally in the colours of the more popular team on the building. Likewise, projectionmapping projects and urban screens around the world regularly promote everything from cars, to clothes, to mobile phones on prominent civic buildings. Overall, the expansion of critical, creative, and commercial uses of expressive architectural surfaces is a growing cultural force that is changing our relationship to iconic, monumental structures.

These new sites of conversation, contestation, and commerce in public culture have two key characteristics. Firstly, they are big-they are massive. As a result of their scale they are highly visible and loaded with significance and thus culturally and economically valuable. They take space, that is, they take up a significant amount of prime real estate and demand to be considered as public and communal. Secondly, they are communicative and 
technical-they are media. They use their scale, visibility, ephemerality, centrality, and communicative capacities, from data visualizations enabled by programmable LED façades, interaction through sensors and mobile ubiquitous media, moving images, sound, and networked communication, to broadcast their messages and engage on- and offline publics. They make space and produce it through interactions both proximal and distal: they mediate. All together, they are massive media.

\section{Rationale for Studying Massive Media}

Architecture and media shape our understandings of who we are, individually and collectively, and change how we read and interpret the world around us. The exploration of creative, historical, and critical understandings of massive media is necessary considering the proliferation of heavily screened architectural environments and their potential impact on the development of public culture and architecture. As such, this dissertation addresses the following questions that seek to better describe, historicize, theorize, and deploy massive media: How do these new assemblages of media, architecture, and space reflect a history of monumentality? That is, how do they reflect and challenge our notions of public culture and how we express collective unity, civic pride, nationalism, and progress? How does massive media change and challenge our notions of space, monumentality, and the public sphere through the application of various primarily screen-based media? How can the combination of media in the form of moving images, data, networks, and animation make large-scale public displays and urban media environments more inclusive and sensitive to their contexts? Furthermore, as an artist, how can I learn more about this form 
and test its limits for criticality by studying art made for these assemblages, investigating their curatorial aspects, and designing and deploying my own installations?

By answering these questions, this investigation yields useful tactics for practitioners and researchers in new media art and the humanities, providing a test for theoretical claims made about the transformative properties of digital technology in cities and for monuments, and a reliable guide and predictor of future outcomes, directions, and critical practice in this field. This work establishes critical perspectives, theories, and methods for the practice of public visual culture through massive media amidst rapid technological, social, epistemological, ontological, and economic change.

To situate this work within a singular academic field is difficult. Fields such as film studies, screen cultures, visual culture, architecture, and urban planning are implicated here and point to the interdisciplinary importance and emergence of this confluence. In fact, this dissertation works towards viewing culture as sitting precisely at the intersection of architecture, digital culture, urban studies, film studies, new media art, and curatorial studies. This dissertation contributes to all of these fields through a close analysis of carefully selected case studies and creation-as-research installations of massive media-an interdisciplinary area of research and practice that has yet to be explored in depth-and develops specific theoretical and methodological lenses that will serve to ground this new field of study.

To begin, I outline three theoretical lenses through which massive media can be viewed: the public sphere, monumentality, and space. In the next section, I will address each in turn while describing the ways that media have impacted each theoretical category 
which I will expand upon and illustrate in case studies and creation-as-research examples in the subsequent chapters.

\section{Media and the Public Sphere}

The concept of the public sphere is directly related to monuments and media; in fact, it is very much implied within them. The public sphere can be seen to have undergone a trajectory of transformation and fragmentation alongside technological advancement. Jürgen Habermas (1974) defines a healthy public sphere as the place(s) where private people come together to form a public that is as accessible, autonomous, non-hierarchical, and participatory as possible. Habermas' understanding of the contribution that various media and spaces have on this coming together is crucial to the resulting qualities of the discourse generated by a public sphere. He notes that the public sphere, due to its size, is a dispersed commonality of strangers, which "requires specific means for transmitting information and influencing those who receive it" (49). For the bourgeois public sphere, emerging in the early eighteenth century from a previous courtly conception of "representative publicness" (Habermas 1989, 5), this meant a combination of media and public space that included the growth of the press, literary societies, the salon, and the coffee house. Habermas argues that the health of the bourgeois public sphere diminished in the twentieth century due to a re-feudalization and fragmentation via mass media that concentrated power in large multi-national corporations and isolated individuals in private dwellings where the media platforms of television and radio had taken hold. 
Newer concepts of the public sphere begin to conceptualize the role of media in the public sphere. Following from Habermas, German filmmaker and theorist Alexander Kluge and his colleague Oskar Negt take on the challenges posed by mass media to the health of the public sphere such as fragmentation, isolation, and distraction. Negt and Kluge (Hansen 1993) were particularly interested in electronic media and satellite links that created the conditions for the existence of global news outlets such as CNN in the 1980s. Instead of celebrating the global proliferation of electronic media as McLuhan did, Negt and Kluge saw "the media of industrial commercial publicity, in their most negative implications, as an inescapable horizon, and as the most advanced site of struggle over the organization of everyday experience which contextualizes all other sites" (211). Thus, the public sphere became for them a struggle for the contextualization of sites of debate and memory through media. As a result, their approach shifted the conditions for the health of the public sphere to those of "openness, inclusiveness, multiplicity, heterogeneity, unpredictability, conflict, contradiction, and difference" (189) that can be enacted in and through the use and appropriation of media which changes the who, what, and how of participatory politics. As Miriam Hansen (1993) notes in an essay that revisits Negt and Kluge's work:

The new types of publicity that have been proliferating over the past decade or two, especially with the electronic media, not only urge us to rethink, once again, the function, scope, and mode of intellectual activity; they also force us to redefine the spatial, territorial, and geopolitical parameters of the public sphere. (183)

The proliferation of media in public space via monumental projections and displays, as well as networked, location, and context aware technologies, can be seen to 
introduce a new dimension to the ongoing struggle for spaces and forums for reasoned argument and debate. Here, specifically, Claire Bishop (2004) is critical of the quality and meaning of the modes of public participation afforded by certain assemblages of expressive displays, digital sensors, and networked devices as public spheres. In her discussion of relational aesthetics and the idea of activated spectatorship in installation art, Bishop argues that good participation should open up channels for discussion and debate. Bishop notes "the public sphere remains democratic insofar as its naturalized exclusions are taken into account and made open to contestation" (65). In the context of public space, these "naturalized exclusions" include the exclusions of sociability enforced by the prioritizing of flow through spaces as well as the prohibitions related to the rights of the citizen to alter their surroundings or see themselves reflected back in it. For Bishop, what is central to authentic participation is "activated spectatorship" $(2006,50)$, or a scenario that values equality over quality, collective authorship over singular control, and the "on-going struggle to find artistic equivalents of political positions" $(2012,3)$. The degree to which various examples of massive media achieve this kind of "activated spectatorship" will be discussed in this dissertation.

Finally, Michael Warner (2005) adds the perspective that the contemporary public sphere can deal in more than just reasoned argument. It can also be apolitical and agnostic, emotional and playful. It can be merely about getting involved intimately, viscerally, and deeply with strangers. This could be something as simple as cheering at a sporting event, collectively facing (or de-facing) a monument, retweeting a hashtag en masse, or clicking a "like" button online. All of this points to a politics of visibility, a "distribution of the 
sensible" (Rancière 2000) and presence that is supported and challenged by various extensions and tools such as the expressive surfaces of structures and media linked in and through them via media interfaces. In this way, massive media emerges as a kind of "common" (Hardt 2009), a public sphere that serves to define dominant economic and social uses of public space. In this dissertation, I will consider how massive media are responsible for coordinating a relationship between technology, politics, and economics, constituting public spheres and a commons "oriented towards the production of social relations and forms of life" (Hardt 2009, 26).

\section{Monumentality}

Like the public sphere, monumentality is a concept that has been tied up in the politics of memorialization, debate, and representation, and is one that has been influenced and inflected by various technologies of production and communication, thus challenging and changing notions of space, public space, and the public sphere. Monuments are dense transfer points that collectivize individuals and condense memory and ideological meanings which are then, through the monument, transferred back to the collective through the performance of rites and rituals associated with them. Analyzing this in greater detail, Henri Lefebvre (1997) argues that monuments in general enact two major things: "(1) displacement, implying metonymy, the shift from part to whole, and contiguity; and (2) condensation, involving substitution, metaphor and similarity" (136). For example, with respect to displacement, the Statue of Liberty can be a stand-in for the whole of the United States. As another example, The Empire State Building can represent New York as 
a whole, and to come in contact with it or its image is to come in contact with the entire city in one place or image. Conversely, with respect to condensation, New Yorkers, or even Americans as a whole, can congregate around these objects (or image-objects)-they condense attention, values (life, liberty, and the pursuit of happiness), and longing for connection into a dense point of semiotic transfer. In this dissertation, I explore how the addition of social media to these sites extends the possibilities for trans-local displacement and condensation through telepresence.

Monuments can be any combination of material, symbolic, and functional characteristics. Pierre Nora (1989) uses the more general term lieux de mémoire, or sites of memory, and includes anything from a state archive to commemorative statues and plinths and even shared moments of silence which we might observe on Remembrance Day as possible sites of memory. He says these are necessary because we no longer have milieux de mémoire, that is, "real environments of memory," (7) a category of collective memory lost in the post-industrial decline of peasant societies in which memory was contained in "gestures and habits, in skills passed down by unspoken traditions, in the body's inherent selfknowledge, in unstudied reflexes and ingrained memories" (12). That said, Diana Taylor (2007) argues that the distinction is not as clear as Nora contends. Taylor places a greater emphasis not on temporal shifts, but in changes in forms of transmission, which may be embodied or archival and may occur among different kinds of publics and communities (22), thus modulating the qualities of cultural survival and affect in memory. The Arc de Triomphe, as a site of memory for example, honours those who fought and died for France in the French Revolution and Napoleonic wars, or, with the recent projected application of 
the text "PARIS EST CHARLIE," also honours those who died in the terrorist attacks on the Charlie Hebdo office (Keromnes 2015), indicating the collective importance of this issue to Parisians, the French public, and via its remediation, the rest of the world. While the Arc de Triomphe is a site of memory, in Nora's words, it is also a more nuanced form of cultural transmission in that it combines forms of transmission through the application of projection (the stone monument and the projected image) while also reaching communities locally and globally in its remediation-another form of transmission. In the Arc de Triomphe, like all sites of memory, the material, symbolic, and functional coalesce as collective memory that, in Nora's (1989) words, "crystalizes and secretes itself" (7), and does so today through multiple, composite, and simultaneous forms of transmission.

While Nora emphasizes the variations in possible sites of memory, and Taylor refines this by emphasizing the role of media, it is the specificity of large, public displaysthe plinth, the arch, the statue, or the clock tower, illuminated, animated, or not-that represent a unique trope of monumentality that is particularly germane to the study of massive media. It is both the scale of the display and its urban situation in these examples, often one that is both visible and central, that lends them particular powers and influence. Monuments and urban space effectively act cooperatively as power brokers that exchange authority and importance through metonymy (literally and figuratively: what and who they stand for) and condensation (who and what they call out to congregate around them). Michael Rowlands and Christopher Tilley (2006), quoting Herbert Bayer, note that these "civic compositions... assume that the urban landscape is the emblematic embodiment of power and memory" (500). Monuments represent this power and memory in relation to 
their setting, and do so in concentrated form. They are concise statements of memory and power within the urban landscape as a whole. In this way, monuments and landscapes give power and significance to one another, one existing on the stage of the other, made particularly visible to their audience in their scale and position: they produce and share what Walter Benjamin (1999) might call the auratic. The Empire State Building in New York City provides one such example. Situated as it is in central Manhattan, the backdrop of the city with its layers of power and memory coalesce with the monument due to its scale and juxtaposition with that backdrop. The images of the building, promotional and personal, reproduce this idea as a testament to the aura produced by it (as opposed to diluting it). The various reproductions of the building's images are inspired by and contribute to its unique geographical situation. Monuments exist prominently within urban and public space so that both the monument and the space around it can benefit from the metonymy described by Lefebvre (1997), allowing for a transfer and amplification of power.

Not only do monuments transfer meaning, they also transfer power through meaning for those that control and direct their construction and use. As Lefebvre notes: ... each monumental space becomes the metaphorical and quasi-metaphysical underpinning of a society, this by virtue of a play of substitutions in which the religious and political realms symbolically (and ceremonially) exchange attributes-the attributes of power; in this way the authority of the sacred and the sacred aspect of authority are transferred back and forth, mutually reinforcing one another in the process. $(1997,136)$

The authority to facilitate the construction of the monument is seen as sacred, and this reverence is recursively transferred with the "religious" (or ideological) content of the monument which has its own symbolic power in society. Thus, power is consolidated and 
shared by form and content. The dispositif-to borrow a term from cinema studies-of the monument in the city (its visibility, scale, and permanence) represents an attractive and useful site for discourses of power because of the metaphorical and metaphysical connection it creates between power, authority, and society. For example, Big Ben, a unique source of identification for the City of London, symbolizes the orderliness of parliament and reinforces the power and centrality-geographically, politically, and symbolically-of the institution, which then, recursively, becomes a source of pride. The authority of order, and the orderliness of authority are broadcast, transferred, and mutually reinforced. Monuments have metaphorical and metaphysical power to which societal values, civic and national identities, and political ideologies are attached, exchanged, and entrenched.

In addition to a transfer of power, monuments also act as dense transfer points for memory and connection when coupled with rites and rituals that animate the space around and on them. As Rolwands and Tilley $(2006,500)$ point out, monuments “... provide stability and a degree of permanence through the collective remembering of an event, person or sacrifice around which public rites can be organized" (500). Monuments, such as war memorials or iconic, city-defining structures such as the Eiffel Tower produce a set of associated social practices such as annual Remembrance Day rituals or marriage proposals. As Lefebvre (1997) notes, "Such a space is determined by what may take place there, and consequently by what may not take place there (prescribed/proscribed, scene/obscene)" (135). This includes mediated practices such as the use of recording devices, the socially accepted volume of speech, the calendar of rites and rituals associated 
with the site, the laying of wreathes on certain days, and so on. Essentially, Lefebvre's point is that monumental space is produced through proscribed performance. Take, for example, the many war memorials in the form of statues or more abstract concrete, metal, or marble forms, in front of which wreathes are placed at specific times of the year. This practice of monumentality need not be visual either. We might also include the example of the ancient "call to prayer" that rings out across the rooftops of cities in the Islamic world, or the ringing of church bells in the West, both performed from purpose built structures that rise above the space around it, creating a broadcast, so to speak. In this way, sites of memory are completed and maintained through public performance. To this, we can add the performance of the monument itself, or the performances of various kinds of participation it exhibits, when it is animated by computer-controlled pixels in the form of an LED façade or digital projection. Again, this dissertation considers how performance and repetition of rites and rituals help to produce identities for spaces and for people, making them feel permanent and connected through the perceived permanence and symbolic value of the monument.

\section{The Modern Monument}

A shift in the relationship between monuments and their publics can be traced to changes in architecture, economics, and design that reach back to twentieth-century discourses and practices of modernism; namely industrialization, mechanization, electrification, increased travel, nationalism, and the acceleration of capital (Berman 1982). While crosses on hills, spires, and other conspicuous religious symbols formed the majority of the ritualistic monumental landscape prior to the advent of modernity, the last two 
hundred years of monumentality has tended towards more secular structures, many of which incorporate some form or expression of technology and/or media that reflects discourses and practices of modernity.

Monuments of modernity include the Eiffel Tower, a monument to industrialization, the clock tower such as Big Ben, a massive timepiece for the mechanization of industry and standardization of social practice, the great gateways of travel such as The Golden Gate Bridge or the Statue of Liberty, welcoming weary immigrants to a new nation, or the sky scraper such as the Empire State Building with its electric lights lit by the energy of capital made to circulate at ever increasing speeds by the occupants that light its windows. These were monuments to capital, to standardization, to consolidated power, to functionalism, but not necessarily to any specific population, group, or historical event. Like modernity, they were impersonal, efficient symbols of hope and progress. While modernity realized itself through mechanization, the circulation of capital, and even cinema, as Hansen (1995) points out, it is also realized through monumental expressions of mechanical, sculptural, and electric media.

According to certain modernists, these monuments, while emblematic of their time, still left much to be desired in terms of the role that monumentality should play. For example, Sigfried Giedion theorized that monuments should be sites for "collective emotional events, where the people play as important a role as the spectacle itself, and where a unity of the architectural background, the people and the symbols conveyed by the spectacles will arise" (568). This was not the case with the kinds of monuments modernity had produced. Giedion called his views the "new monumentality" (Mumford 2000, 151), 
essentially arguing that monuments should not be stoic and stuffy, but should allow collectives to participate in and through them in some way. While acknowledging the return to grandeur at large scales in public spaces which characterized modernist monumentality, in Nine Points on Monumentality (Giedion, Léger, and Sert, 1943), members of the Congrès International d'Architecture Moderne (CIAM) which included Giedion and the French artist Fernand Léger, advocated instead for something more responsive and variable in monuments. Tellingly, their suggestions included the provision of "vast surfaces" for projection:

Mobile elements can constantly vary the aspect of the buildings. These mobile elements, changing positions and casting different shadows when acted upon by wind or machinery, can be the source of new architectural effects... During night hours, colour and forms can be projected on vast surfaces for purposes of publicity or propaganda. These buildings would have large plane surfaces planned for this purpose, surfaces which are non-existent today. (50)

Giedion et. al saw the provision of colour, light, and variability as a progression in monumentality: a redefinition of grandeur, identification, and monumentality through surface contingency. Along with Moholy-Nagy, whose experiments with his Light-Space Modulator at the time mirrored the aesthetic goals of CIAM, and were even extended by him (theoretically) to the realm of architecture, these modernists, critiquing modernism, suggested that there was potential in architectural dynamism for "publicity and propaganda" through the application of moving light. How this would create greater emotional connections between people and space was not clear at the time, but the potential had been identified: monuments needed to become more like screens to become more engaging and break from modern and pre-modern precedents. 


\section{Space and Media}

A further understanding of theories of space and media is necessary for the development of critical and creative concepts for the study and use of massive media. As was shown in a number of the examples above, space and monumentality are not static or fixed but produced in complex social and technical processes. This idea comes to us primarily through Lefebvre (1991). In his The Production of Space, he argues that we have moved past a strictly Euclidian, mathematical understanding of space that sees it as measurable, continuous, and discrete, to an understanding of space as a collection of fragmented, subjective spaces produced through various practices, protocols, social relations, and technical supports. As such, spaces need not be purely physical. They can include mental spaces, commercial spaces, global spaces; essentially there are multitudes of spaces $(1991,236)$ that are produced and also, importantly, consumed in time through our actions. While in a public square, for example, we simultaneously consume and produce the space by photographing it, appropriating it in various ways, or transposing it to other spaces and contexts through media. A public square is a commercial space in that various exchanges and transformations of capital are present in the form of advertisements, street vendors, and even street photography. It is also a global space in the way these photographs might circulate in print or online, or the way in which the materials and goods on display are made and marketed elsewhere. Finally, it can be a mental space in the personal cartographies created by individuals, something that Kevin Lynch (1960) describes in his Image of the City, or in the way Nora (1989) describes sites of memory. Space, instead of being fixed, is co-produced in the complex interplay of social and technical actors, groups 
and individuals, matter and memory. Take the specific example of being in a crowd in Times Square: the bright lights and grand vistas of the space contribute to its production, as well as tourists snapping photographs (which contribute to mental images for those near and far), the nearby hotels cashing in on the spectacle, and the advertisers reaping the attention lavished upon the flashy billboards by the eyeballs that scan them.

Technologized spaces such as Times Square manifest themselves in the many relations that are opened up when space is not seen as authentically achieved, static, or absolute. In his book The Media City, Scott McQuire (2008) uses the term relational space (28) to define this shift as it relates specifically to theories of contemporary public spaces. By way of television, cinema, and photography, and now accelerated and augmented by media including digital mobile devices and urban screens, public space has increasingly become something that, according to McQuire, "cannot be defined by essential attributes or inherent and stable qualities" (22). He argues that these media factors have introduced greater ambivalence and contingency to our conception and use of space. McQuire explains that changes in urban form and the embodied experience of space beginning with the modernization of cities, the development of rapid transport, and the electrification of cities in the early twentieth century diminished the coherence of traditional means for the representation and construction of social space and identity. Public space, once solidly "anthropological" (Lefebvre 1991, 229), based on tradition and familiarity, became increasingly unfamiliar, defined instead by the flow of people and capital (Lefebvre 1991), and now, information. 
Changes in how space is produced have also changed how space is perceived and how people reorient themselves amidst these jarring changes. As McQuire notes, "in a world remade by machine technology, artificial light and rapid movement, embodied perception was increasingly susceptible to sudden switches and abrupt shifts" (62). Consequently, changes in urban form "levied increased demands on technological images to 'map' the city, and thereby make it available to perception, cognition and action" (ix). Thus, the city and media have existed in a dialectic between rupture and recuperation, between distraction and enhanced attention. For example, serialized photographs taken of the Haussmannization of Paris, a process that introduced a great deal more artificial light and rapid movement to the city, were used to both demonstrate the transformation of spaces as well as to make it comprehensible. Similarly, the radical montage of early city symphony films such as Dziga Vertov's Man With a Movie Camera (1929) had the effect, as Benjamin observed, of unlocking the "prison-world" of the modern metropolis (quoted in McQuire, 65) that had become incomprehensible in its complexity and the speed with which it was to be traversed by car, tram, or subway.

Furthermore, McQuire demonstrates how distinctions between private and public, local and global, are blurred to create new spaces, and the ways that communicational and spatial bonds are transformed as a result. Expanding on the quality of ambivalence of contemporary relational spaces, McQuire (2008) notes that "relational space names the ambivalent spatial configuration which emerges as the taken-for-granted nature of social space is withdrawn in favour of the active constitution of heterogeneous spatial connections linking the intimate to the global" (ix). The role of mobile ubiquitous media 
in public is a clear example of this shifting spatial characteristic. Smart phones can, for example, aid in the escape from a taken-for-granted context such as public transit by providing refuge through an intimate (yet potentially globally connected) space. The enclosures that once defined private and public, local and global, recede and hybridize in such instances. As a consequence, McQuire argues, space today more frequently assumes significance through heterogeneous, variable, and impermanent interconnections (22) as opposed to unidirectional, homogeneous, fixed connections (as envisioned in early practices related to television consumption, for example). As McQuire concludes, "communicational bonds exhibit different durations and velocities to older forms of social bonds embedded in spatial proximity" (22) and thus, relational space contains an ambivalence that comes with the speed associated with the rapid switching of telecommunication, and forms the basis for the mixture of contexts, of spaces of places (Lefebvre 1991) and spaces of flows (Castells 2001, 2002).

In a concept similar to McQuire's relational space, Adriana De Souza e Silva (2006) focuses on the role of mobile interfaces in creating what she calls hybrid space (261). This also serves to describe the space in which massive media emerges as both a disruption and a filter. "Produced and embedded by social practices, in which the support infrastructure is composed of networks of mobile technology" (271), hybrid spaces blur the boundaries of physical and digital space, augmenting both in the process. Data is extracted from these spaces through practices such as surveillance, personal photography, or social media, while also augmenting these spaces via computer displays and public screens. As De Souza e Sliva notes, "the flows of information that previously occurred mainly in cyberspace can now be 
perceived as flowing into and out of physical space" (265). Like McQuire, De Souza e Silva considers our "always-on" connection to virtual spaces in physical spaces as the means by which remote contexts, both in space and time, become enfolded inside the present context (262), creating an ontological bridge of sorts. McQuire adds a critical dimension to the idea of enfolded, hybrid, and ambivalent space noting that these spaces create mostly hidden meshworks of surveillance evoking what Deleuze (1992) presciently described as an emerging "control space." At the same time, these hybrid spaces also contribute to pockets of network-driven activity such as flash mobs and protests, and furnish access to buildingscale effects on urban screens and reactive architecture via interactive installations. As McQuire notes, this can lead to a perpetual state of responsiveness and anticipation for devices, individuals, and buildings-akin to computer interfaces themselves-that act either as distractions or a points of grounding reference.

McQuire (2008) names contingency as a second major characteristic of the emergence of relational spaces. He notes that both public screens and mobile devices contribute to a fluidity of social space and subjectivity that can be considered radically contingent particularly with the speed at which images are produced and consumed within these contexts. The contingencies brought about by the mixture of spaces and contexts have, for McQuire, allowed for the emergence of new spatial ensembles that better reflect the ways we interact in and through the image today. For example, the current apex of the heterogeneity and contingency of spatial regimes can be seen to be embodied in practices such as augmented reality and mixed reality (21) in applications such as Google Glass and Microsoft's HoloLens. In the promotional discourse attached to such devices, and in 
McQuire's theories, there remains an optimism about a new frontier of hybrid space in terms of the freedoms it affords for the layering of contextually relevant information, for embodied yet telepresent encounters, but also for dissenting voices and interpretations, something that the artistic examples of Lozano Hemmer and Wodiczko help to elucidate. In addition, contingency, as a characteristic of contemporary relational space, can be seen as central to the affective register and thus, much like cinema before it, a powerful attraction for representational (and commercial) applications and perceptual training through spectatorship and participation. The moving and networked image in public space plays a significant role in the accelerated contingency of surface effects-the multiplication of images in, on, and around buildings as a result of public and private displays-and the attraction/distraction that this creates. Sigfried Kracauer (1965), an early observer of the effects of contingency introduced into space by the moving image, argues in his Theory of Film that cinema works through the distraction and mystification caused by the moving image. This is precisely a distraction from the surrounding environment and context of the observer. Kracauer observes that film results in an increased demand on the spectators sensorium resulting in a distracted attention, innate curiosity, and an openness to sense impressions. The moving image creates an effect that captivates an audience and acts as a unique and powerful physiological stimulus. He likens film to an object of prey, tapping into our animalistic tendencies to notice and fixate on moving objects. Janet Harbord (2007) extends this analysis to public spaces. She argues that contingency is central to the affective register and notes that whereas films projected in cinemas can be described as contingent insofar as the moving cameras that captured them present changing views 
within a cinematic frame, current configurations of expanded cinema via projections and screens in public space can achieve contingency and the subsequent effects of distraction, attention, and mystification through indeterminate viewing conditions and digital manipulation that open the digital moving image to recombination, relationality, and reactivity. Public screens up the ante in terms of the distraction/attention dialectic of media, a dialectic that will be explored in more detail throughout the dissertation.

Following from this, properties of cinema such as scale (Doane 2009), superimposition, montage, and apparatus/dispositif (Baudry 1975) are important factors in considering the implications for the combination of media and monumentality. Tom Gunning speaks about the role that superimposition plays in cinema and beyond in his article, "To Scan A Ghost: The Ontology of Mediated Vision” (2007). He notes that "incongruous juxtaposition" of a superimposition on film "... yields an eerie image of the encounter of two ontologically separate worlds" (6). Although, in Gunning's case, he is describing the appearance of superimpositions upon and within the diegetic space, when this diegetic space is the life world, so to speak, then the superimposition becomes the projection itself and the thing being imposed upon is the city, opening up new ontological complications and possibilities. Furthermore, public projection can be seen to extend the concept of montage, the juxtaposition of images to create new meanings, to a spatial montage with the city around it again, affording new possibilities for the moving image and space. Both of these concepts can be encapsulated within an understanding of the apparatus/dispositif, the ideological and technical interface of cinema, as expanded (Youngblood 1970) and extended by massive media. For example, Mary Ann Doane 
(2009), writing on the role that scale plays in cinema, describes the way that the cinematic scale reflects a desire to lose oneself in the image-an essential quality for ideological transmission through any media form achieved to a degree by the dispositif. These concepts of film and cinematic media take on new dimensions and qualities in the massive projections and animated displays of massive media.

\section{Accelerated Rituals of Space and Media}

One potentially negative side of the increased stimulus of contingency, often by way of the application of media in public space, emerges in the belief that it can erase history, mystifying and fragmenting a public for the purposes of capturing an audience within the crosshairs of ideology. Jonathan Crary argues that the "accelerated ritual" (Crary 1999, 370) of the moving image can efface the history of a space and divorces repetition from its association with tradition building, that is, the repetition we might associate with seeing the same structure every day, for example. As Crary argues, electrification transformed the surfaces of the city into mutable, oneiric, "formless fields of attraction" (468) that often had an effect of distraction and dehistoricization for the purposes of pleasure and persuasion. That is not to say that tradition, community, and debate cannot be fostered through the moving image in public space. In a world dominated by moving images, tradition may be found simply in the repetition of the spectatorship of the moving image, or it may be created in repeated exposure to the same images or sets of images, or perhaps in their appropriation and circulation amongst viewers. McQuire (2008) characterizes such practices as moving us from "object-oriented perception" (41) to a mode of perception that 
foregrounds the relations between objects and operations on them-essentially, to a mode of perception based on ideas of superimposition and montage that emerge from the creation and reception of cinema. Thus, in relational space, tradition, connection, and meaning is not completely obliterated, it simply takes on a different velocity: it is more ambivalent and contingent, but no less meaningful. Extending this thinking to mobile screens, McQuire also notes that in contingent, relational space, "the pre-given nature of social space and the taken-for-granted contours of subjectivity are increasingly withdrawn in favour of the ambivalence of mobile spatial configurations and ephemeral individual choices" (22) engaged by such devices. As such, contingency is seen to be suspended in relational space, albeit briefly, through the "interconnections established between different nodes and sectors" (22) and thus tradition and connection may be re-established, albeit primarily according to this modified logic of the networked screen. Rituals, publics, and tradition are not necessarily obliterated by screens-they are made temporary and provisional, site and device specific.

Thus, screen technologies in public spaces might be seen as both distractions and filters, making space more and less sensible and legible. Picking up on the way mobile technology modifies the legibility and flows of public space and changes power dynamics, De Souza e Silva and Frith (2012) focus on the filtering possibilities that personal media afford us, noting the ways they provide a mast to affix ourselves to amidst increased contingencies of media maelstroms that now permeate public spaces. Similarly, media artist David Rokeby (1995) has stated that "we are desperate for filters" (154) in the sense that we need ways to manage the contingency of media messages and channels that we actively and 
passively encounter. While technologies such as mobile phones and urban screens can be seen as contributing to contingencies, they are also the filters by which these contingencies can be navigated and managed for the purposes of legibility. Similar to Simmel's (1950) concerns decades earlier, De Souza e Silva and Frith cite the omnipresent threat of overstimulation by the city and its associated media through interfaces both individual (such as the smart phone) and shared (such as public space) as ultimately desensitizing. De Souza e Silva and Frith see personal, mobile interfaces as connecting, influencing, and providing some means of control over flows and spaces of information. These interfaces, and the growing importance of the concept of interface in culture, address a growing desire to selectively interact with public spaces as a way to fend off the onrush of a blasé attitude that might result via the bombardment of the senses. As such, mobile media, alongside the massive media they accompany directly or indirectly, are capable of making public space both more and less legible and sensible.

Predating McQuire, others such as Moholy-Nagy have taken a positive stance on the subject of increased stimulus in the city and its relationship to perceptual acuity. As he states in Painting Photography Film, his investigation into perception and various media, "The vast development both of technique and of the big cities have increased the capacity of our perceptual organs for simultaneous acoustical and optical activity" $(1969,43)$. He cites Berliners crossing the Potzdamer Platz who simultaneously perceive "the horns of the motor-cars, the bells of the trams, the tooting of the omnibuses, the halloos of the coachmen, the roar of the underground railway, the shouts of the newspaper sellers, the sounds of a loudspeaker" (43). To close the loop, he insists that far from being a 
mechanism for disorientation, this shift in stimulus can prove generative for the media arts where "modern optics and acoustics, employed as means of artistic creation, can be accepted by and can enrich only those who are receptive to the times in which they live" (43). Arguably, what this groundwork of media studies shows is how media and processes of mediation make the modernizing city more manageable and unmanageable, and how the human organism is co-evolutionary with its media rich environment, something that is echoed in the writings of Benjamin and McLuhan. This points us towards posing important questions about the role that massive media play in this dialectic of perceptual rupture and recuperation, questions that I tackle in the following chapters.

Others, such as Brougher (2008) in The Cinema Effect, further argue that the illuminated night of the modernized city, with its moving images and the growing cultural obsession with the moving image, triggered an ontological shift in city life beyond mere perception. As Brougher notes, “... film has spilled out of the great movie palace cathedrals and has spread into the city itself and into the way we live our lives" (19). He notes that since the advent of outdoor projection, amusement parks, and other such illuminated spectacles, we no longer encounter the cinema's technology of spectatorship, its apparatus and dispositif, in the cinema alone. As he says, “...dark chambers separated from the world at large are no longer a necessity for the cinema effect" (35). These alternative cinema-like scenarios can furnish the possibility of alternative worlds and spaces of reverie amidst the very real, material spaces of everyday public life, thus engendering the possibilities for abject negation of one's surroundings and/or increased relationality, hybridity, and legibility. 
Increasingly, the gap both perceptually and ontologically between cinema and the city, between mediation and the built environment, has narrowed due to the proliferation of cinema and the city's increasing uptake of the functional characteristics of cinema, namely the use of electric light in darkened spaces filled with people. McQuire (2008) and others (Gunning 2006) actually situate a mobility of vision and an embodied, collective spectatorship in proto-cinematic environments such as the World's Fairs and Expositions of the late nineteenth century. As McQuire (2008) notes, "The World's Fairs showcased the potential for electric lighting to establish a new rhetoric of urban space, opening the way for the city to be transformed into a performative space in which fixity of appearances would give way to increasing flux" (119). Similarly, Gunning (2006) states that "The World Expositions were not only founded on the regimes of the wandering eye, but they also proved to be expert tutors in the delights (and possible perils) of this new mobile vision" (15). Perhaps one of the most well-known cinematic cityscapes that embodies this vision is that of Times Square in New York City. Critical theorist Marshall Berman (2001), in his article "Metamorphoses of Times Square," observes of the space that “... you have to tie yourself to some sort of inner mast in order not to be overwhelmed" (42). Whereas the traditional cinematic experience provides a mast in the form of a comfortable, standardized environment of spectatorship, the media city affords no such reliable reassurances or restraints. The exaggerated electronic forms of Times Square turn the city into a stage, complete with lights, camera, and plenty of action. As Berman notes, "Under the lights a whole new world could be born... the street itself is a form of living theatre; ordinary people on the street are performers as well as spectators" (54). It is within this frenetic, 
frameless context that massive media experiences can be seen to operate, and in which their possibilities for the construction of new registers of space and identity are played out.

To aid in an understanding of such affective, fragmented, relational, and mediatized spaces, the concepts of the "composite dispositif" (Verhoeff 2012), "nonrepresentational space" (Thrift 2008), the "peripatetic" audience (Bennett 2009), and "transversal" identity (Murphie 2004) will prove useful. First, composite dispositif describes a state of mediation in which standardized environments of spectatorship, such as the cinema, the city, or the interface of a smartphone, overlap and co-constitute a relational experience. This concept helps to provide a more nuanced and complex understanding of media as being semi-coordinated, filter-like, and personally constructed, based on access to various technical and visual interfaces (such as the city and mobile devices). Here, Verhoeff draws from both Michel Foucault (1980) and Jean-Louis Baudry (1975) in constructing her sense of what dispositif means. For Foucault, dispositif is used to describe a historically specific mixture of material and discursive practices that combine to contribute to some form of social control (i.e., a prison), while Baudry, following from Louis Althusser, uses the term to describe the conceptual arrangement that interpellates the viewer into a certain subjectivity or point of view as a result of a coordinated technical apparatus. In cinema, this coordinated technical apparatus is the equipment, such as cameras, film, the theatre space, and other cinematic hardware required to produce various effects, namely the capturing and maintenance of the viewer's attention on the diegesis. For massive media, this technical apparatus might include elements of cinema, architecture, urban space, mobile technologies, and telecommunication. As such, the effects of massive media can be 
considered as composite, relational, and contingent mixtures of many technical assemblages and associated dispositif. For example, the coordinated effect of a heavily screened environment that includes elements of the media city such as urban screens and mobile devices that cater to an ambulatory spectator that is variously attracted and distracted by media $(2012,104)$ might be described as a composite dispositif. Simply put, the composite dispositif describes a situation in which we are both captured and comforted, distracted and attracted, by the overlapping media layers that define relational space. This is an important concept when considered alongside Margaret Bennett's concept of the "peripatetic" audience of performance in public space which, she suggests, must be captured by embracing and supporting multiple, incomplete combinations of media forms (12).

Massive media also alludes to the possibility of expressing another concept of contemporary spaces known as "non-representational" space (Thrift 2008). Thrift suggests that metaphors of cyberspace and information superhighways have challenged the ideation of what constitutes environments for experiences and action in contemporary life-of space. Nigel Thrift focuses (2006) on flow as opposed to what he calls "authentically achieved space" (141) existing outside of the of-the-moment flows of people and things in and through it. In this, he shares an affinity with Lefebvre in that he foregrounds action, performance, and flux in the production of space. Thrift's work also has parallels with Manuel Castell's $(2001,2002)$ notion of space as being the result of tensions between the "space of flows" of the electronic age and what he calls the "space of places" (576). The inclusion (and incursion) of this "space of flows," the electronic, computerized networks of 
telecommunications, into the "space of places," the physical nodes of public squares, skylines, roads, neighbourhoods, and buildings-the lines, nodes, landmarks, and zones that Lynch (1960) describes in his Image of the City-is an important shift in how we conceptualize space. Spaces of places have become globalized through technological incursions, and spaces of flows are localized when substantiated through technology (such as screens, personal and public) in spaces of places.

Such a techno-social situation can be seen to produce what Andrew Murphie (2004) designates as "transversal" subjectivities existing in many localities, or trans-locally. To understand the identity of a peripatetic entity as transversal within a composite dispositif in non-relational space is to understand it not as transcendent or fragmented, but as deeply enmeshed with other identities and locations. One phenomenon that demonstrates the way identity is expressed and performed transversally today is seen in the way different profiles may be acted upon on multiple websites for various purposes. Trans-

local, transversal identity may also be produced through urban screens that are networked or participatory, once again, merging spaces of flows and spaces of places, generating more rich and varied hybrid spaces and publics.

\section{Entering Supermodernism}

Taken together, this transformation in what space is and how it is constructed points to a shifting architectonics of space, that is, the expression through architecture of the history, setting, and specificity of a site (Frampton 1983), one that continues to be shaped primarily by various visual media and the flows they engage and carry. To put a 
name to these changes, theorists have invoked terms such as super-(Ibelings 2002) or hyper- (Augé 1995) modern architecture and space. Supermodernism describes a design perspective focused on and inflected by the acceleration of technology in the design, creation, and reception of architecture, space, and the monumental. While these spaces might include the non-descript spaces of automated flow in the service of capital, such as airports and parking garages (according to Marc Augé), they also include architecture, such as the programmable tip of the Empire State Building, that enacts and embodies an information aesthetic (Manovich 2008) through specific and precise computer visualizations and animations.

As with attention and legibility, the digital can be both a blow and a boon to architectonics, depending on who you ask. Paul Virilio (1986), writing somewhat exasperatingly yet presciently in the late 1980 's, a time when the challenges and possibilities of the digital were just beginning to be felt, argues that the digital can sever our connection to the material and corporeal. Virilio sees the dawn of supermodernity as a distinct shock to the very principle of architectonics, and thus also to the ability "to assert, describe, and inscribe reality" (18). He notes:

In fact, if architectonics used to measure itself against the scale of geology, against the tectonics of natural reliefs with pyramids, towers and other neogothic structures, today it no longer measures itself against anything except state-of-art technologies, whose dizzying prowess exiles all of us from the terrestrial horizon. (30)

One example might be the critique of architectural works such as Frank Gehry's Bilbao Guggenheim Museum, criticized for semiotic and technical overreliance on the algorithmic instead of purely architectonic considerations. McQuire (2008) argues that "Gehry's project 
utilized the computer not as a tool for communication but as a technique of architectural mastery" (98), thus creating a structure that has a greater connection to the tools of its conception than to site-specificity. What Virilio warns us of, and what is exemplified in the example of Gehry, is the danger of a kind of technological determinism in architecture and monumentality that ignores the communicative potential of these new technologies.

Hal Foster (2010) argues that to make architecture more "actual, grounded and pragmatic" (136) we cannot ignore technology. Instead we need to find ways to have it express the "social real" (136), which includes the traces of culture, memory, and presence of the site and the people that populate it. In many ways, this echoes the sentiments of the CIAM in their advocacy for a greater connection to the emotions of people and the space(s) they inhabit. For Foster, expressing the social real includes coming to terms with what he calls our "complicity in the culture of the image," meaning that our buildings must work both as powerful images and as generators of bodily affect, thus reflecting a shift in our thinking regarding the relationship between architecture, mediation (technology, or image), and affect (embodied experience). The opposition between the terrestrial plane upon which buildings and bodies are measured, the plane of appearances (images and buildings themselves), and the non-representational (information), is continually collapsing and should be expressed and developed materially in architecture and public space. One way to do this is to create "urban visualizations" with expressive architecture that are considerate of the environment (surrounding buildings, local culture, atmosphere), content (the information displayed and any interpretations that may be generated by it), and carrier (the building, square, façade, or any other element that supports the broadcast medium) 
(Vande Moere and Hill 2012, Vande Moere and Wouters 2012). The proliferation of massive media might be evaluated based on how well it is situated in a real-world environment (both borrowing and contributing to it), how informative it is (whether it allows onlookers to create meaningful insights or provide feedback), and if it provides a useful civic function (is aesthetically pleasing while calling for some kind of reflection, change, or action).

Finally, as Hito Steyerl argues, our complicity in the culture of the image in general, but specifically through our changing hybrid surroundings, also calls to mind the importance of "post-production" (quoted in Shental 2013) or the documentation, dissemination, and archiving that occurs officially and unofficially alongside the production of culture which can be seen to contribute to a supermodernism in architecture and culture. As Steyerl notes, "We are embedded within a post-cinema that has been completely transformed, mutated into whole environments, permeating reality to the point that we can now understand it with media thought and alter it via post-production." Supermodern architecture may be seen as a conduit or catalyst for the circulation of images in that it contributes to a reality understood through remediation and the personal recombination and distribution of images and media traces.

\section{Studying Massive Media}

Based on the theoretical and historical bases discussed above with respect to media, space, monumentality, and the public sphere, this dissertation analyzes case studies (Yin 2012) and deploys and reflects upon creation-as-research (Chapman and Sawchuck 2012) 
projects in order to form a deeper understanding of the past, present, and future of massive media.

Creation-as-research is employed to engage directly with the material, believing that to truly understand one's subject, one needs to become immersed in it, to struggle with the logistical, theoretical, and creative questions that inevitably arise. Throughout a total of five creation-as-research projects that include a number of large-scale public projections and public data visualization installations, I provide a point of comparison against the case studies presented in the chapters of this dissertation, as well as a specific perspective of the artistic and curatorial use of massive media. Here I take creation-as-research to be a subset of research-creation, the more general term for the integration of a creative process, experimental aesthetic component, or artistic work as part of a research work (Chapman and Sawchuk 5, 2012). Creation-as-research (19) specifically relates to the "elaboration of projects where creation is required in order for research to emerge" (19). In this case, while research is the end goal, the results produced also include the outcomes of creative production. As Chapman and Sawchuk note:

It is about understanding the technologies/media/practices that we discuss as communication scholars (for instance) by actually deploying these phenomena, and pushing them into creative directions. It is a form of directed exploration through creative processes that includes experimentation, but also analysis, critique, and a profound engagement with theory and questions of method. (19)

I have designed, proposed, situated, and experienced the technics (Thain 2008), politics, and practices associated with massive media, using these experiences to augment analysis, critique, and theory. In this way, creation-as-research follows from Heidegger's thinking about "praxical knowledge" (Smith and Dean 2009, 7) where ideas and theory are 
ultimately the result of practice. As Matthew Fuller (2005) notes, commenting on the challenges of observing and understanding the imbrication of media and spatial assemblages, or media ecologies: "to see how the world operates necessitates a more complex and involved participation in it" (106).

In terms of case studies, I use Yin's (2012) definition of a case study as a contemporary phenomenon set within a real world context whose investigation is used to uncover a deeper understanding of it and the meanings associated with it. The major case studies of this dissertation probe particular contemporary instances of massive media to flesh out their inflections and possible trajectories, all in relation to a rich and varied history of media and architecture. Each chapter includes a short literature review section and focuses on one of three related yet distinct areas of massive media: moving images in large-scale public projection, data-responsive low-resolution façades, and the curation and programming of massive media.

In Chapter 2, I investigate the narrative and associative potential of massive media with respect to moving images. I focus primarily on Robert Lepage's Le Moulin á Images (or The Image Mill) (2008-2012), McLarena (2014) at the Quartier des spectacles, and two creation-as-research projects: 30 moons many hands (2013) and The Line (2013). The Image Mill, presented in the lower town of Quebec City every night in the summer from 2008 to 2012, is the world's largest outdoor projection to date. This nightly occurrence celebrated the $400^{\text {th }}$ anniversary of Quebec City by presenting a mixture of archival footage, motion graphics, light, and sound on a 300m wide wall of grain silos in the lower town of the city. With purposefully dimmed street lights, the 'stadium' seating provided by the city's 
ramparts, and a radio signal that carried audio to viewers in hotel rooms surrounding the site, this presentation actively transformed the city into a cinema through a composite dispostif (Verhoeff 2012) centred upon its massive display. In addition to The Image Mill, I also evaluate McLarena, an interactive outdoor projection at the Quartier des spectacles in Montreal that incorporated the direct participation of its audience. Through conversations with the lead production designer of The Image Mill, a close reading of documentation (video and catalogue), producers' reflections about the project, and site visits for both examples, I compare and contrast the approaches and outcomes of these massive outdoor projection-based installations and read them through the theoretical lenses of the public sphere, spectatorship, monumentality, and space as inflected by media. I argue that the expanded cinema of monumental projection places the moving image in a direct relationship with its surroundings, thus invoking an extra-diegetic, spatial montage with the city, multiplying contingency and ambivalence and thus extending the boundaries and characteristics of architecture and monumentality. I also argue that cinematic superimposition persists in large-scale public projection and is extended through the urban and architectural specificity of the substrate and situation in which it is presented. These new architectures and affordances of massive media spectatorship mediate between space, people, and image, recentering audiences towards a confluence of bodies, the city, and discourses of memory. Taken together, the special effects of cinema taken outdoors transform the conditions of space, monumentality, and the public sphere, furnishing possibilities that range from "dehistoricization" (Crary 1999) to "activated spectatorship" (Bishop 2004) in public spaces. 
Building upon these principles, Chapter 3 focuses primarily on low-resolution LED façades, highlighting their unique role as public data visualizations and the specificities of this more durable and architecturally integrated form of massive media. Specifically, I examine the narrative and associative potential of The Empire State Building. A structure that has a long history of architectural lighting, the Empire State Building upgraded its lighting to a programmable LED façade in 2012 providing animation capabilities and greatly accelerating the rate at which the lights and the causes they represent are changed. The Empire State Building, as a case study, elucidates the connection between dataresponsive low-resolution façades, monumentality, and space. The addition of programmable lighting extends its existing function as symbol and monument of the city by integrating it into a daily program that sees it oscillate from highly corporate advertising programs to the commemoration of a much more diverse set of functions, causes, affiliations, and events. These include displaying political campaign information in realtime and dynamic animations set to live music broadcast over the radio, all disseminated and circulated through various channels such as television and social media. A closer look at this particular assemblage, its history (and that of related structures such as weather beacons), the discourses associated with it, and the role that data visualization plays on large, low-resolution façades, demonstrates the way such structures couple with concepts of supermodernity (Ibelings 2002), hybrid (De Souza e Silva 2006) and relational space (McQuire 2008), and “new monumentality" (Mumford 2000, 151). Two research creation projects, In The Air, Tonight (2014-), a project created for the LED façade of the Ryerson Image Arts Building in Toronto, and E-TOWER (2010), a project created for Toronto's CN 
Tower, further demonstrate that low-resolution media façades and public data visualizations, in their massive embodiment of data of shared consequence and the subsequent intensity of embodiment experienced by spectators and participants against the scale and significance of urban space, better embody the development of a supermodernism (Ibelings 2002) in architecture characterized by the irruption and imbrication of the "infoscape" and the cityscape, where data-rich public spaces of identity, congregation, and contestation seek and find appropriate and consistent outlets in highly visible, contingent, and ambivalent (McQuire 2008) spatial assemblages of architecture and media. I also argue that low-resolution media façades must be situated, informative, and functional while considering their carrier, content, and environment (Vande Moere and Wouters 2012, Vande Moere and Hill 2012) which must be expanded to consider on- and off-line spaces. They must do so, also, within the difficulties of annotation and context associated with low-resolution displays and the challenges of gatekeeping organizations that regulate what can be displayed.

Finally, without informed curatorial support that guides, develops, and fosters artistic experimentation and opportunity for massive media, the findings above remain futile, as they will never be able to be sustainably actualized. Thus, Chapter 4 looks at the roles and responsibilities of artists and curators in signalling the way forward in art and public culture through massive media. Here, I focus on two particular efforts to develop spaces, sites, and practices of massive media. The first is the EU funded group Connecting Cities, which aims to build up a worldwide connected infrastructure of media façades, urban screens, and projection sites to circulate artistic and social content. The second case 
concerns the New York-based Streaming Museum, an organization that forms temporary partnerships with cultural and commercial centres to produce contemporary-themed art exhibitions on screens (including its website) and public spaces on seven continents. Through conversations with Susa Pop and Nina Colossi, key curators and producers from Connecting Cities and Streaming Museum respectively, and an examination of their curatorial methods and goals, I describe the careful selection and coordination of on- and offline sites and networks of production and presentation and the provision of experimental relational practices in diverse urban contexts that they employ. Exhibitions such as Streaming Museum's Nordic Outbreak which included works that circulated online and on seven continents, including a month-long multi-screen presentation of Björk's Mutual Core in Times Square, and Connecting Cities' Binoculars to... Binoculars from..., an extended voyeuristic feedback loop between multiple European public screen scenarios, provide examples of the curatorial challenges and opportunities of massive media with respect to access, audience, participation, spectacle, and scale. I augment this analysis by presenting an on-going creation-as-research project that seeks to open up the Ryerson Image Arts Building LED façade to greater artistic experimentation through institutional change and the provision of technical protocols and support. Taken together, these studies indicate a broader shift in curatorial attention from autonomous artworks to curatorial networks, hybrid infrastructure, transfer protocols, technical specifications, and software packages that is particularly relevant to massive media. They also demonstrate that negotiations with corporate and civic entities are crucial in order to find solutions for artistic practices of massive media that are sustainable and suitably autonomous. 
Furthermore, beyond selection and production, curating massive media involves a responsibility for harnessing complex dispositives and the complexities of trans-local space for aesthetic critique and empathetic social connection through large-scale outdoor projections, programmable LED façades, and urban screens. 
CHAPTER 2: Experiments in Large-scale Projection and the (New) New Monumentality

\section{Introduction}

In the summer of 2008, Quebec City became the site of the world's largest architectural projection: a 45-min long projection-mapped spectacle covering 400 years of settlement, development, and culture dating back to when the city was first founded as a French colony. For approximately 60 nights every summer, from 2008 until 2012, street lights were dimmed and people would gather on the docks, along the ramparts, or in hotel rooms around the city to witness an iconic building, the massive BUNGE grain silos in Quebec City's harbour, tell the story of this UNESCO world heritage site (UNESCO 2014). With each screening attracting about 10,000 spectators, Le Moulin a Images (The Image Mill) by director Robert Lepage and his team at Ex Machina (see Figure 2 and Figure 3) was seen by about half a million people every year (Dubé 2012, 187).

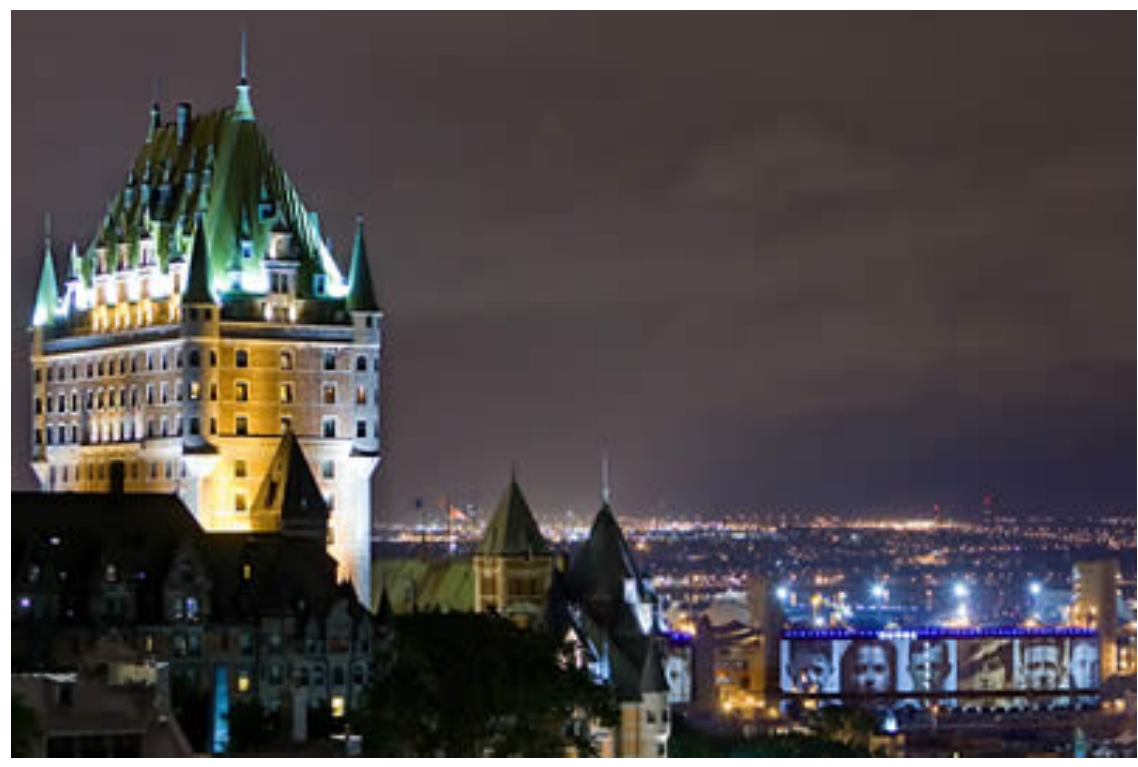

Figure 2. Robert Lepage / Ex Machina, The Image Mill (2008), with the Chateau Frontenac in the foreground, Quebec City. Photo: Christie/Francis Vachon. 


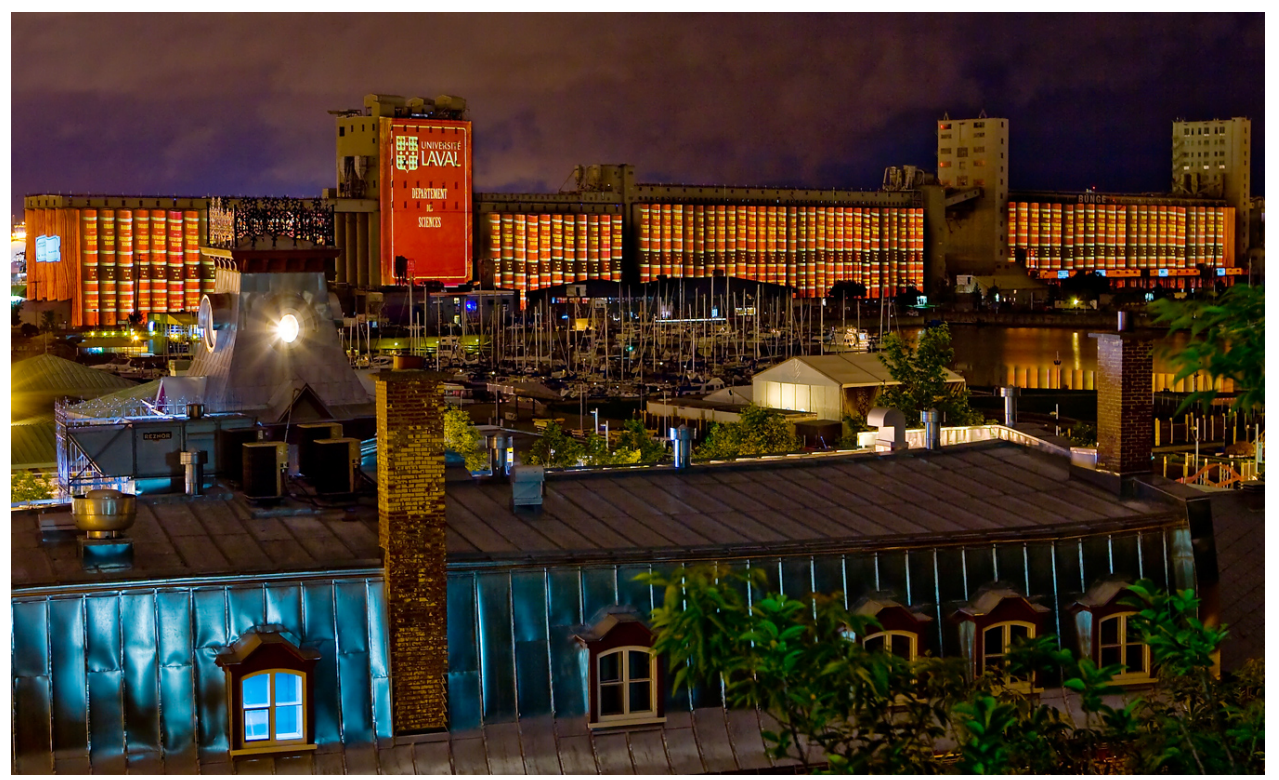

Figure 3. Robert Lepage / Ex Machina, The Image Mill (2008), from the lower town with boats in the harbour, Quebec City.

The Quartier des spectacles in Montreal represents another approach to the presentation of historical material in public space through large-scale projection. One particular installation, McLarena (2014) (see Figure 4), an interactive public projection commissioned by the National Film Board of Canada, invited participants to mimic the choreography of the dancer in Norman McLaren's Canon (1964) in a mobile recording unit located in front of the projection. These images were presented in the style of Norman McLaren's film on the façade of a building outside the Saint-Laurent metro station. 


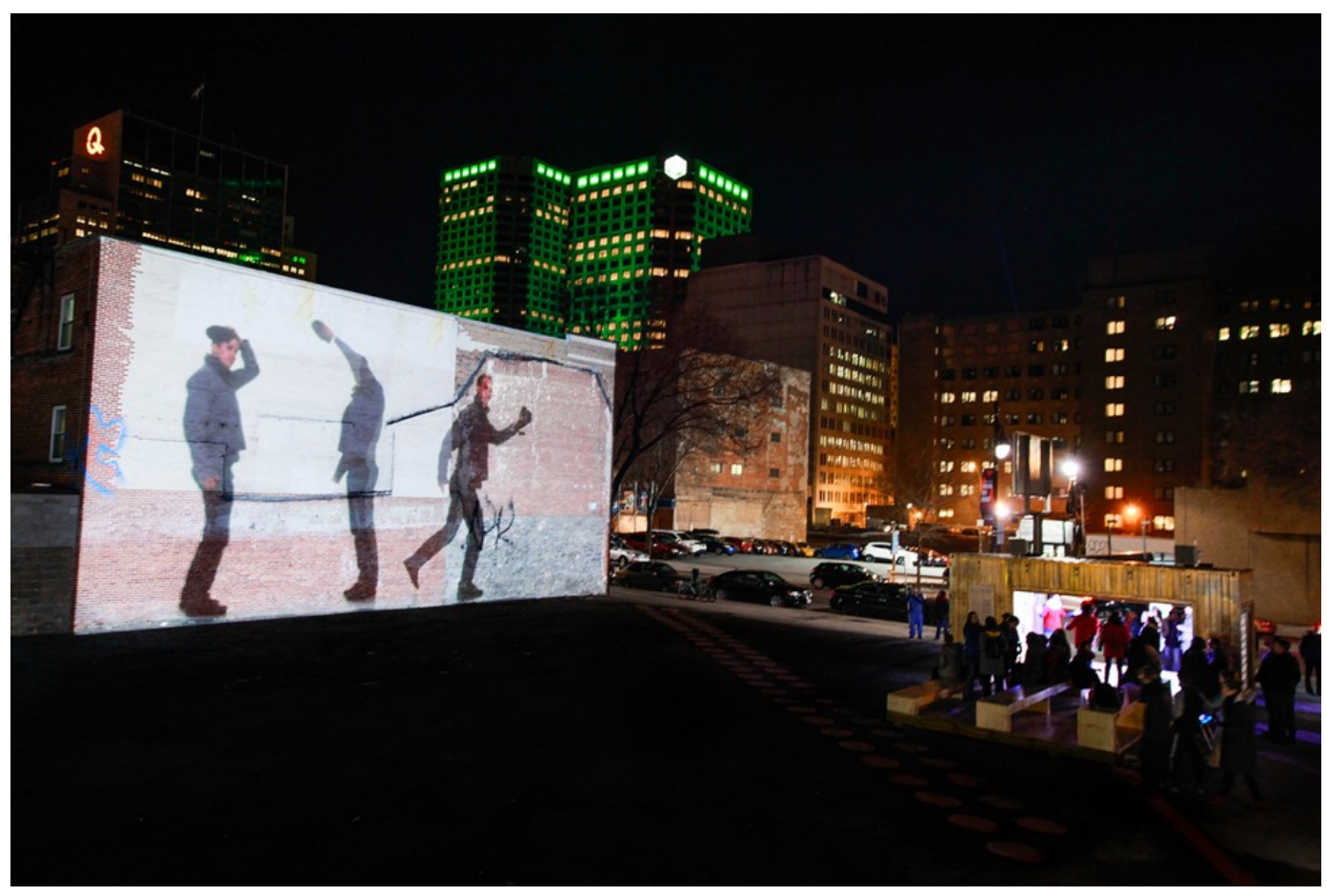

Figure 4. Daily tous les jours, McLarena (2014), Quartiers des spectacles, Montreal.

Through historical research, site visits, participant observation, and close readings of documentation, press clippings, and literature related to both The Image Mill and McLarena, I argue that large-scale applications of the moving image change and challenge concepts of space, monumentality, and the public sphere, instituting a more participatory, moving image-centred public culture in which we identify and engage with collective presence, memory, and action through information, architecture, and the moving image. I demonstrate the ways that an expanded cinema, one that merges both the logic of the monument and the logic of media and the spaces of flows and spaces of places, unlocks the narrative and associative potentials for the moving image and public space. In addition to this, through two research-creation projects-30 moons many hands (2013) and The Line (2013)-I operationalize these theories while describing the ways that massive media can 
enable urban activism and debate, and new telematic rituals that straddle the critical, affective, and commercial. I also describe how institutional ideologies limit artistic autonomy as well as the development of audiences, sites, and access-the development of spectatorship, historical discourse, and a public sphere based on the communal, large-scale moving image. Finally, I argue for the importance of repetition and semi-permanence that allows for greater syncretism to site and memory of situated, historical information presented through massive media that can help to develop a new "new monumentality." In this chapter, I argue that massive media contribute to the production of hybrid (De Souza e Silva 2006) and relational (McQuire 2008) space by focussing on three key elements of moving image culture and production: superimposition, montage, and dispositif/apparatus. These concepts are mined for their affective efficacy in massive media while also stretched and transformed in their unique application in public space. The expanded cinema of monumental projection places the moving image in a direct relationship with its surroundings, thus invoking an extra-diegetic, spatial montage with the city, multiplying contingency and ambivalence, and thus extending the boundaries and characteristics of architecture and monumentality. Spatial montage considers the moving image with the contingent spaces around it-with the city-and creates new contingent semiotic reference points in a massively expanded cinema. I argue that superimposition, an effect of cinema that serves to complicate ontological conditions, both in the diegesis and for the spectator, persists in large-scale public projection and is extended through the urban and architectural specificity of the substrate and situation in which it is presented. Finally, and perhaps most importantly, the key considerations of apparatus and dispositif form an 
enduring touchstone for the experience and effect of technical assemblages in the service of certain affects and ideological discourses which range from the democratic to the commercial. The new architectures and affordances of massive media spectatorship mediate between space, people, and image, recentering audiences towards a confluence of bodies, the city, and discourses of memory. Taken together, these special effects of cinema taken outdoors create a composite dispositif (Verhoeff 2012) that helps to transform the conditions of space, monumentality, and the public sphere, furnishing possibilities that range from "dehistoricization" (Crary 1999) to "activated spectatorship" (Bishop 2004) in public spaces. Massive media can produce public spheres characterized by an oscillation between fragmentation, cohesion, conflict, and commercialization, and new registers of monumentality (Mumford 2000) that are intended to better reflect contemporary social and community life and more effectively capture and transfer power, memory, and connection. The application of media to architecture in the form of large-scale, (sometimes) interactive moving images reflects a shift towards a more participatory, moving image-centred public culture in which we identify with collective presence and memory through the moving image.

\section{A Short History of the Moving Image in Public Space}

The application of the ephemeral moving image in public space has always left an impression. In a particularly telling example, in a letter home to his family, Sigmund Freud describes his visit to Rome's Piazza Colonna (see Figure 5) in 1907, a site that had been transformed for him due to an early foray into outdoor projection. In the centuries-old 
square, Freud witnesses "lantern slides," "short cinematographic performances," and advertisements projected on a screen at the end of the piazza (Crary 1999, 363). In a letter, written in his room later that evening, Freud is unable to recall if the Piazza contained a fountain, nor does he mention the significance of the monumental column for which the space is named. Freud is, in his own words, "spellbound" (370) in front of the cinematograph and unable to recall additional information about the space. Crary argues that Freud's amnesia suggests public projection is a harbinger of "a dehistoricized perpetual present" (369), dulling the ability to recall or engage with anything beyond the moving image as it subsumes its audience.

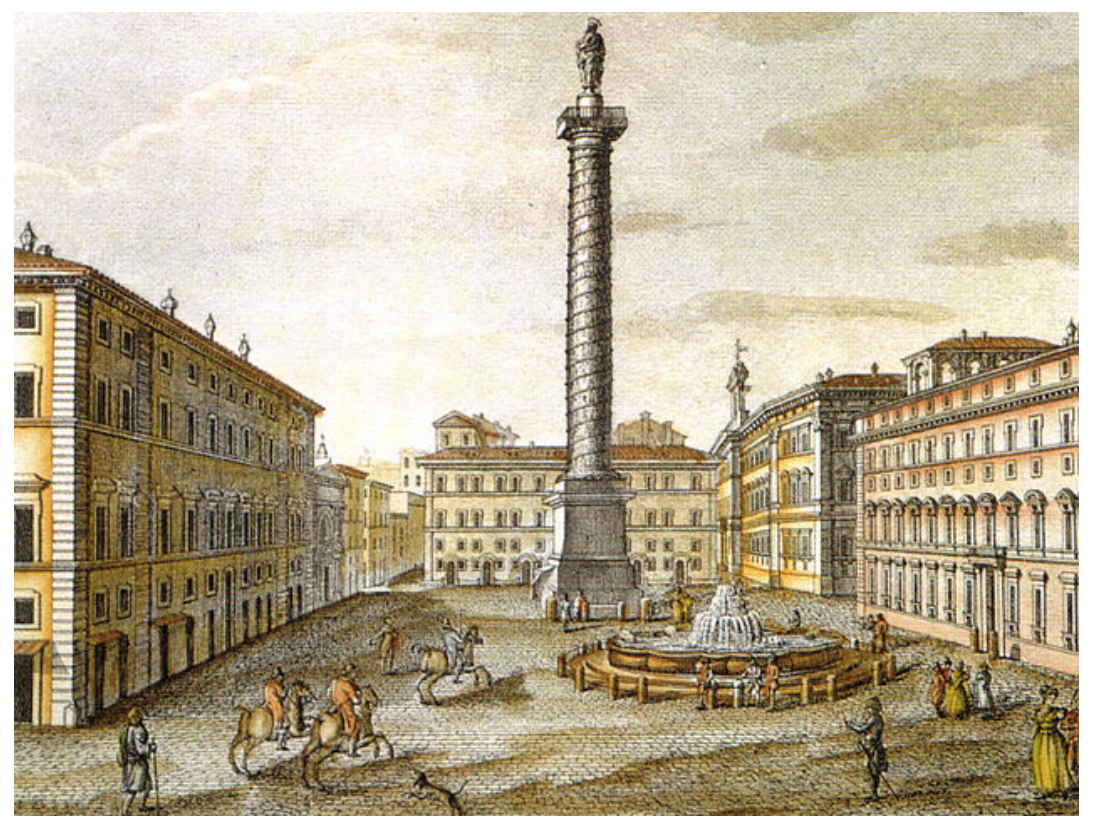

Figure 5. Illustration of the Piazza Colonna, Rome.

This effacement by way of moving images in public space was seen to continue throughout the twentieth century. Electrification throughout the twentieth century 
transformed the surfaces of the city into mutable, oneiric, "formless fields of attraction" (Crary 1999, 468) that often had an effect of distraction and dehistoricization for the purposes of pleasure and persuasion. Hubs of public moving image culture, such as New York's Times Square and London’s Piccadilly Circus, became sites of performance, spectacle, pleasure, and disorientation (Berman 2001). They were scenarios for directing attention that merged the cinematic with the architectural in monumental proportions to produce novel effects that complimented manipulative rituals related to the consumption of objects, images, and spaces.

The effect that Freud experiences and Crary describes is consistent with, and perhaps predicated upon, the general effect of cinema observed by early theorists of cinematic reception. Cinema, no matter where it is located, is built to create powerful, affective responses. As noted in Chapter 1, Sigfried Kracauer (1965), in Theory of Film, argues that film's ability to record "reality," reveal hidden aspects of reality or aspects located in other places, and render this reality once again in motion is precisely what captivates an audience and acts as a unique and powerful physiological stimulus. In this way, film is like an object of prey, and we are its predators, releasing our animalistic tendencies in noticing and fixating on moving objects. Film increases the demand on the spectator's sensorium as a result, and produces distracted attention, innate curiosity, and an openness to sense impressions.

In a more contemporary example, an openness to sense impressions by way of cinema was exploited in a big way on the banks of the Thames in London in November of 2011. Looking for a way to direct people's attention to their new Lumia 800 smartphone, 
telecommunications giant Nokia used 16 outdoor projectors to illuminate the Millbank Tower, creating a 120 metre high projection-mapped spectacle synchronized to a live DJ set by deadmau5. Thousands came out to the event while documentation circulated on the Internet through social media and blogs in the weeks following. ${ }^{1}$ The content of the presentation consisted of geometrical shapes and patterns pulsating to the music and graphic visualizations on the building that referenced a virtual, electronic world, paying little attention to the site and surroundings and thus validating Virilio's (1986) fears that technology could in fact serve to estrange us from our terrestrial surroundings. The attention generated by the visceral moving images and sound created a collective unity amongst those present at the event yet engaged in a monumentality through the moving image channelled not towards a historical consciousness but towards the valorization of a new consumer product. This was not a site of memory where collective memory "catalyzes and secretes itself," (Nora 1989, 7), per se, but a site of syncretic co-optation by capital. It was, like the Piazza Colonna, a "formless field of attraction" that "dehistoricized" the space (Crary 1999) by tapping into the affective response generated by the moving image.

Yet, an openness to sense impressions by way of the application of the moving image to architecture need not be deterministic nor distracting from real historical conditions of a place or people. Sometimes, distraction is necessary, as Debord (1999) famously argues, to unsettle and unmask capitalist spectacle. Furthermore, dehistoricization and distraction are not necessarily detrimental to historical consciousness nor are they the only outcome of the layering of flickering images and architecture. The

\footnotetext{
${ }^{1}$ Almost 6 million people have viewed the official Nokia YouTube video of the event to date: http://www.youtube.com/watch?v=SX2Gd-kqV5s.
} 
ambivalence and contingency created by the moving image, as McQuire (2008) notes, can create a relational space pregnant with rhetorical possibilities. The work of Jenny Holzer, whose "truisms" (such as "MONEY CREATES TASTE") have among other formats been presented as large, high-contrast architectural projections (see Figure 6), and the participatory digital graffiti of Graffiti Research Lab that allowed people to "tag" a public façade through a combination of lasers and digital projection, are two examples that demonstrate public projection as a powerful tactic for dissent and dissensus, and for contesting a politics of representation and any access issues inherent in public space through the affect and attention they generate by way of the moving image. By destabilizing and semiotically scrambling a sedimented mode of address-in this case, advertising and/or architecture-these artists attempt to counter the foreclosure of space and place by bureaucratic and capitalistic powers. Both works respond to the perceived loss of the public sphere: one by shouting louder, shining brighter, and doing so in more unexpected places than advertisements, the other by temporarily enabling participation, play, and protest at an architectural scale. 


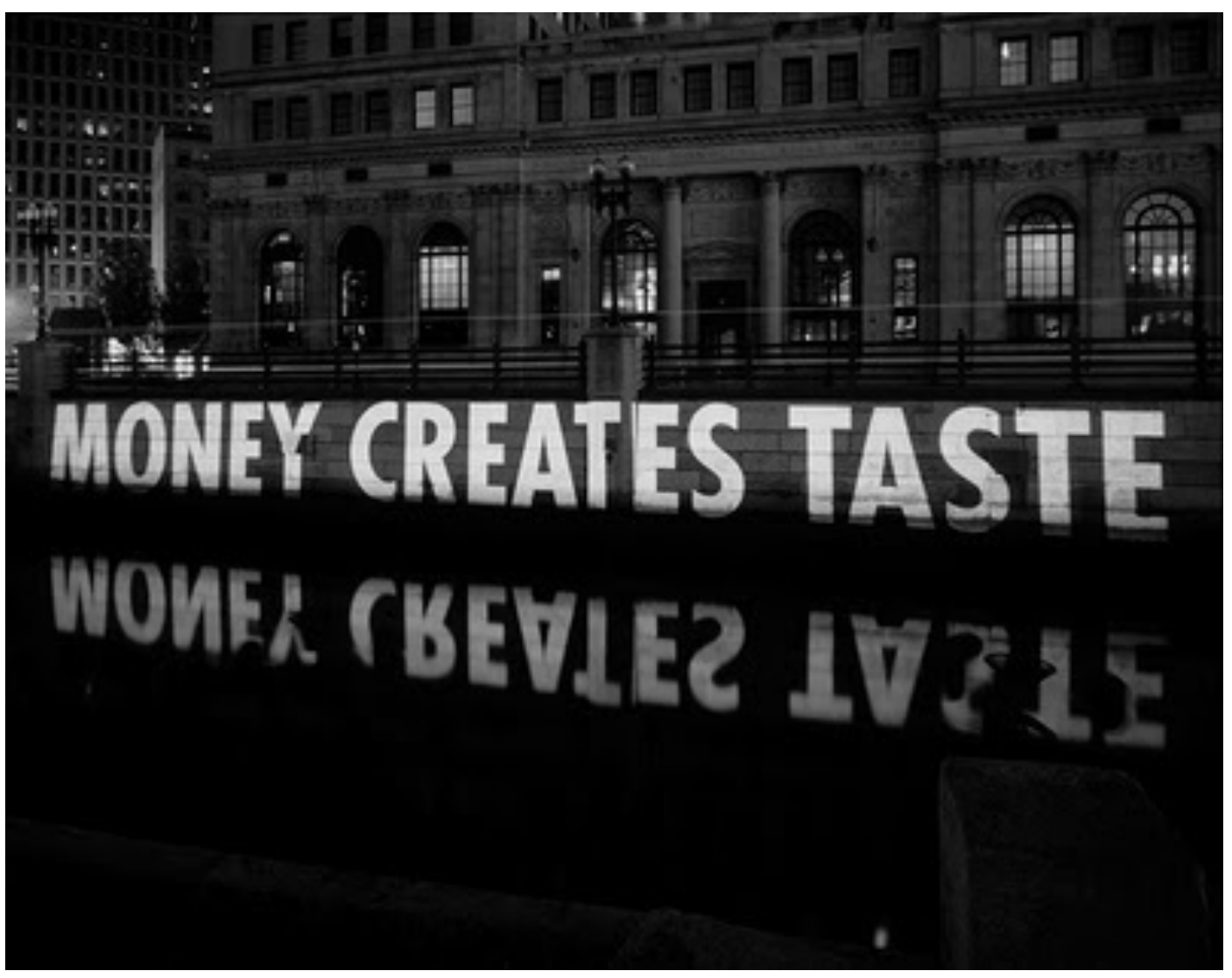

Figure 6. Jenny Holzer, Money Creates Taste (2006), Providence, RI.

Another example, Krzysztof Wodiczko's The Tijuana Projection (2001), involved the projection of video feeds of the faces of abused and exploited women in the local maquiladora industries on the façade of Tijuana's largest art gallery (see Figure 7).

Wodiczko's work uses public projection to draw attention to traumas that affect entire cities and confronts viewers with this information to spark important ongoing processes of social justice which, according to Wodiczko, must be a collective and compassionate endeavour. The collective that forms around a public building, in a public space, becomes a public enunciation in itself: it is part and parcel of the work of public projection. 
Wodiczko (2015) calls this the "outer public," noting that "fearless speaking" requires "fearless listening": "public truth-telling (testimony) and public truth-seeking (witnessing) are interdependent." To stop, watch, and participate in such events implies complicity with the disruptive image and support of its alternative message. The effect of these projections is in fact rehistoricizing; a reinvigoration of a public sphere that challenges what can be said, by whom, and at what scale and level of authority. It engages a politics of aesthetics through a new "distribution of the sensible" (Rancière 2000) made more diverse through the moving image.

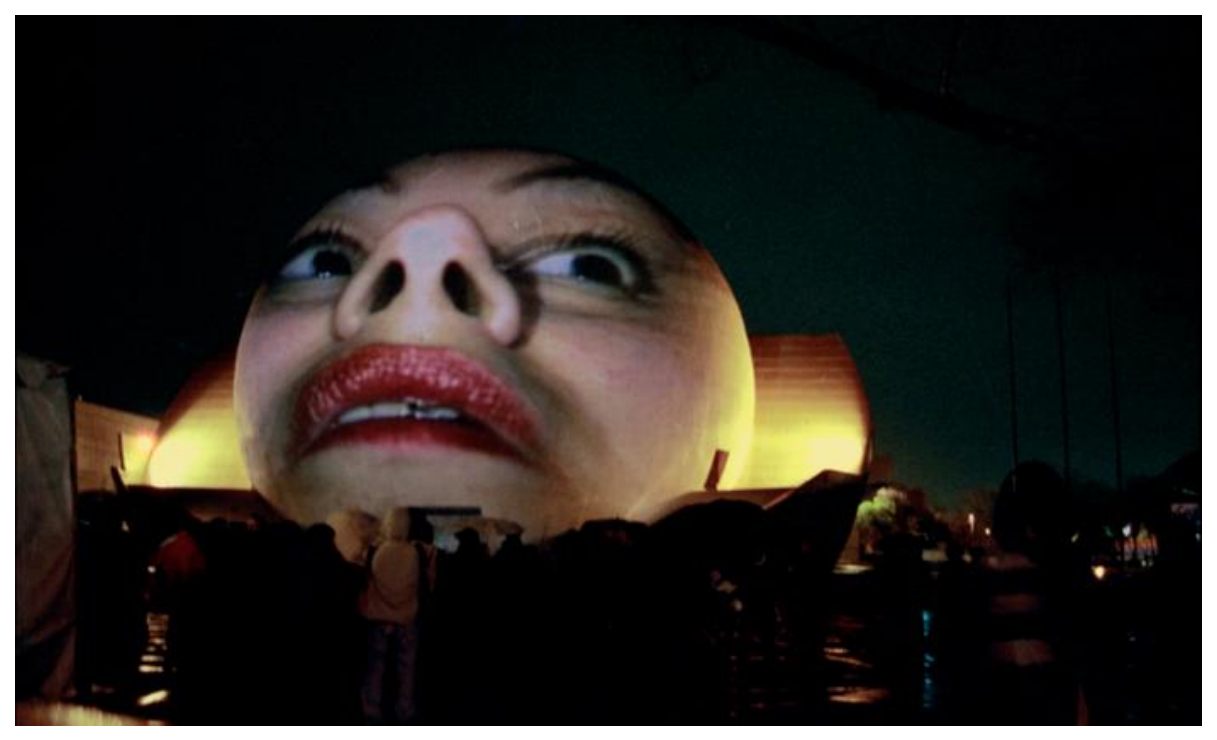

Figure 7. Krzysztof Wodiczko, Tijuana Projection (2001), Tijuana, Mexico.

While the lack of durability of these images may be seen to take away from their power, Wodiczko argues that ephemerality does not necessarily diminish affectivity. As Wodiczko notes (Phillips 2003), a projection, when witnessed by a public, becomes a stain, a mark, a trace upon the memory associated with the building, its surroundings, and the 
various groups it incorporates and addresses. Furthermore, these works, including documentation of the crowds that surround them, are often captured and remediated in books, online, and increasingly in social media, thus legitimizing the gravity of the struggles (or products) highlighted by these events and bolstering their perceived impact. With the Nokia example, documentation was carefully choreographed and created as part of the event, zooming into the crowd taking their own video of the event on their Nokia phones, then to the DJ, and back out across the river to show the giant mobile phone-shaped building "dancing" along with the crowd and the music. This footage was posted on various websites and on YouTube, extending the life, reach, and legitimacy of the event, exemplifying what Hito Steyerl refers to as a culture of "post-production" (quoted in Shental 2013) where images are valued in their ability to circulate and be operated upon. In this instance, the tactics of the commercial world and the art world are indecipherable: they share the same spaces both on- and offline to generate and disseminate their messages. The stain that Wodiczko refers to spreads and mutates with new technologies of image circulation, regardless of the content. The recursion between the stain in memory, the story and image of documentation, and post-production modification is a large part of what legitimizes the massive efforts required to produce ephemeral commercial and countermonumental displays.

A succession of images that implies motion can captivate and confound us, catching us in our tracks, compelling us to remediate it or buy into its message. In a large outdoor projection, the image that sticks to a place in space and a space in memory (individual, collective, and virtual), can create new dimensions of the public sphere in 
which plurality, collectivity, and memory are contested in our shifting conditions of space. While space can be rehistoricized in this context, it can also become dehistoricized, commodified and aestheticized, selling us more stuff, including the image or brand of the city itself. Massive media in the form of public projection represents a new battleground where the "deposit of a memory trace" (Lynch 1960,119) into the hybrid urban environment can make cities more (or less) public.

\section{Architecture, Expanded Cinema, and the New Monumentality}

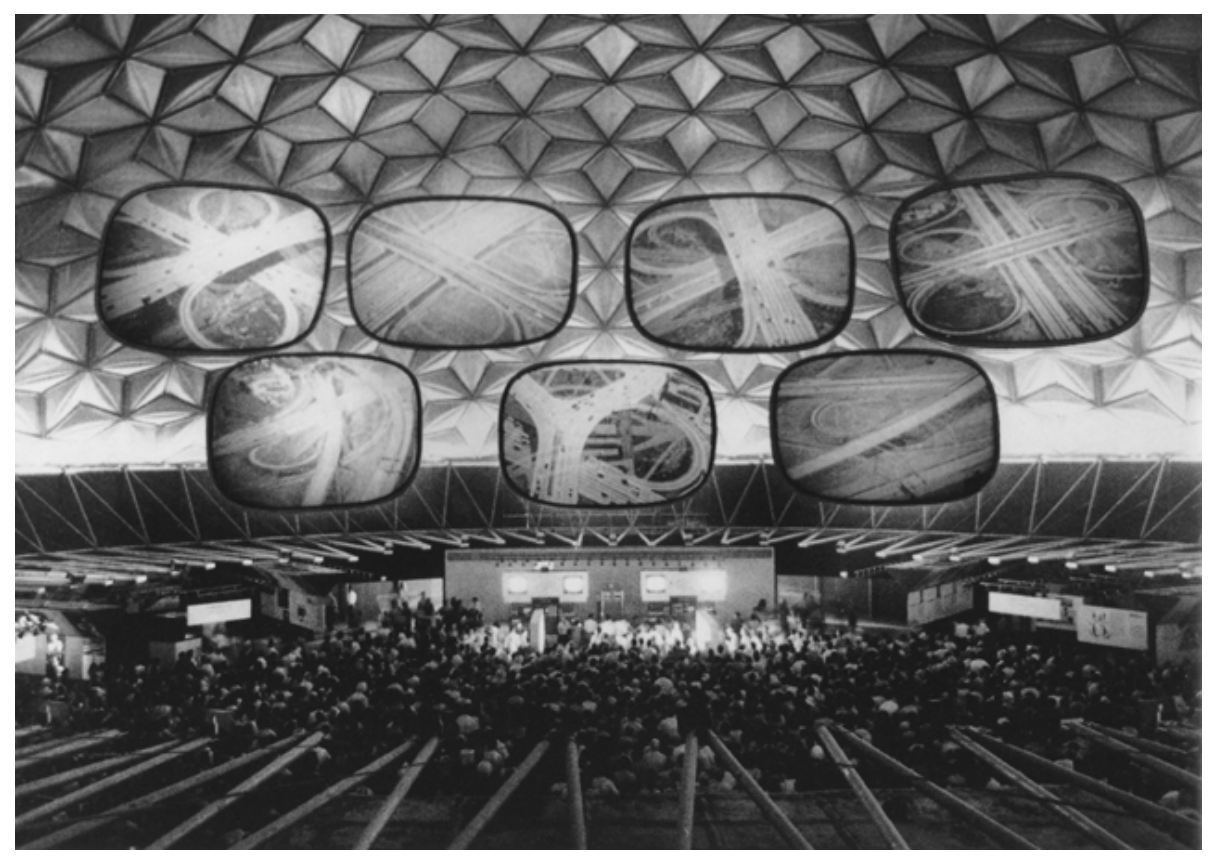

Figure 8. Charles and Ray Eames, Glimpses of the USA (1959), installation view, Moscow.

The experiments and effects of the juxtaposition of architecture and the moving image can also be traced through a lineage that includes expanded cinema, installation art, 
and display culture. For example, the multi-screen experiments and expanded cinema of Charles and Ray Eames showed how the moving image might be integrated and integral to architectural and spatial experience for expository purposes. With Glimpses Of The USA (see Figure 8), the Eameses created a multi-screen expanse within the American pavilion at the American National Exhibition in Moscow in 1959 (Colomina 2009). Situated within a Buckminster Fuller designed geodesic dome and presenting images of highways, bridges, homes, and subdivisions across seven screens spanning approximately four football fields, along with narration and music, the Eames's "screen field" gained much of its monumental impact from its immensity and the interrelationship of multiple images within a space, reinforcing claims of mastery over nature, progress, good design, and culture. It showed the breadth of the technical prowess of the nation within a wider frame of an equally impressive architectural space.

The Eameses demonstrated the importance of the relationality between and outside pictures planes that an architectural juxtaposition of moving images affords. Gene Youngblood (1970) refers to this in terms of expanded cinema, a term that he helped to popularize, where that which lies beyond the frame is included within a multisensory experience that potentially liberates new degrees of creative freedom. In the case of the Eames's work, as well as Wodiczko's projections, the elements outside the picture plane include architectural and civic infrastructure, the presence of the crowd, as well as the location itself and its historical content. Much like the monument, which exchanges power and meaning with its surroundings (Lefebvre 1997), architectural expanded cinema allows for an exchange between moving images and relatively static but no less meaningful 
surroundings, while also explicitly acknowledging and incorporating the presence of the spectator: it makes cinema more public.

While Glimpses illustrated an idealistic expansion of the possibilities for creativity and an expansion of the human sensorium, there was a sense that the expansion of cinema into multi-screen environments was more than just a creative expansion: it was also potentially democratic. Following from Bishop (2012), it could be seen as an artistic equivalent of a political position. For example, Bauhaus pioneer Herbert Bayer's exhibition design for Edward Steichen's Family of Man (1955) at the Museum of Modern Art in New York City employed multiple panels, sizes, and angles of text and imagery in the hopes of developing an "extended field of vision technique" (Turner 2012, 83) that would train viewers to adjust their consumption of images and space providing a so called "democratic" (Turner 2013) means for the viewer to recombine and digest information. The format of the exhibition was such that viewers were to be exposed to a spatial breadth and depth of sensory material and create associative meanings of their own across spatial arrangements, essentially creating their own non-deterministic montage from the expanded apparatus of exhibition. This echoed Moholy-Nagy's (1969) arguments about the importance of multisensory stimulation for the development of perceptual capacity that the expanding metropolis of the early twentieth century was also providing (and requiring) of its inhabitants. Instead of combatting the purported overstimulation, disconnection, and distraction observed by Simmel (1950) and Crary (1999), Bayer, like Moholy-Nagy, embraced contingency and ambivalence in spatial design in the hopes of creating a new political position for spectators-a new register for a public sphere-that was more open, 
heterogeneous, unpredictable, and potentially contradictory (Hansen 1993) so that a more nuanced and personal epistemological focus might be achieved.

The democratic forms suggested by Bayer and the Eameses begin to gesture towards a greater integration of an activated spectatorship with media for democratic and emancipatory purposes. This trajectory, particularly with respect to monumentality, can be seen to have its roots in the writing of Bauhaus architectural pioneers including Sigfried Giedion. Writing in 1944, he asserted that architecture, and in particular monumentalism, had stagnated, or even worse, was failing to respond to the "popular needs and aspirations" (Mumford 2000, 151) of its time. Collective identity, he argued, was not being served by existing sites of memory. The "static immortality" of a world of statue-topped plinths, gothic cathedrals, and the like, did little to reflect contemporary life and the dynamism better embodied in architectural and artistic movements of the day such as cubism, modernism, and futurism. Giedion wondered how monumentality, which by definition was a marker of the condensation and transfer of power and memory (Lefebvre 1997) and which could "seldom be used and then only for the highest purpose" (Giedion 1944, 500), might be better directed towards "the life of the community" (550). While his suggestions lack specifics, they remain prescient nonetheless. For Giedion, monuments "should be the site for collective emotional events, where the people play as important a role as the spectacle itself, and where a unity of the architectural background, the people and the symbols conveyed by the spectacles will arise" (568). Out of this, he called for a "new monumentality" (Mumford 2000, 151) that would restore the connection between citizens and their surroundings that had been lost in the decidedly un-dynamic legacy of 
monuments, a kind of total media environment of memory-a democratic surround (Turner 2013) in public space. The continued expansion of the application of moving images in public space at architectural scales and monumental settings can be seen as an inspired extension of Giedion's enduring questions and worthy of consideration against his lofty claims. The parallels between the potential and perils of mixing moving images, space, and monumentality described above provide a historical lens and lineage through which to understand current experiments in massive media such as The Image Mill and McLarena at the Quartiers des spectacles.

\section{The Image Mill}

Alternatively described by its creators and spectators as akin to fireworks, an art book, outdoor cinema, or even a "kinetic mural" (Dubé 177), The Image Mill (2008-2013) remains the largest "monumental projection show" (Mario Brien, February 9, 2014, phone call with the author) ever made, beating out the previous record set by a $300 \mathrm{~m}$ wide projection on the Egyptian pyramids (Blatchford 2008). The impressive technical specifications for the show are as follows: $600 \mathrm{~m}$ wide, $50 \mathrm{~m}$ high, 40,000,000 pixels (Philips Lighting 2014), 30 video projectors, 30 video servers, 250 lighting devices, 24 JEM fog machines (Bennett 2009), 50 switches and programmable controllers to dim the street lamps around the site, 350 speakers, 50 subwoofers installed beneath the docks, and $20 \mathrm{~km}$ of fibre optics for video, lighting, and sound (Lepage 2011). Spanning the equivalent of 25 IMAX screens (The Canadian Press 2008), The Image Mill was a massive media spectacle 
that incorporated light, sound, and architecture to tell the story of Quebec City on its $400^{\text {th }}$ birthday.

The content of The Image Mill covered various stages of the city's development with the silos transforming into the maps of early explorer Samuel de Champlain, impressionistic scenes of important battles and rapid technological growth, and various cultural touchstones such as a catholic church and the Quebec Bridge. The show begins with images and sounds that make the silos appear as if they are rotating, churning together the images that will cover its surface for the 45 minutes that follow. The sounds of machinery, steam, and whistles recorded from inside the silo can be heard, as Robert Lepage (2011) says, "to give it its own sound, as if it is the building telling its own story." The gears turning also take the viewer back in time, until there is a pervasive darkness except for the twinkling night sky covering the entire surface, as if to make Ezra Pound's claim that with the modern city "we have pulled down the stars to our will" (Quoted in Kenner 1975,5$)$ as literal as possible. The silence and stillness are broken by a Pink Floydesque soundtrack that propels us through images of previous projections-geographic and cartographic-that allowed travellers such as Samuel de Champlain to reach these once foreign shores. Wooden fences rise up across the surface, referencing the wooden palisades and Jesuit forts built just a few hundred metres from the projection site. On the larger tower block that interrupts the long string of silos, we see central figures such as Louis XIV who reigned over both France and New France, establishing a royal government and a settlement policy. The story moves through impressionistic accounts of the St. Lawrence River cracking open in the spring, the importation of the first horse and the acceleration of 
cultural and technological change, and the rituals and beliefs of the catholic church. The building becomes a tableau of votive candles-luminescence mimicking luminescence once again, except this time making a small thing very large-with the sound of church bells ringing in the distance, bringing a kind of rhythm to daily life. As the diegesis takes us forward through history, we witness early battles between the British and French, the presence of First Nations, the mixing of cultures, and the development of fine art and architecture that reshaped the city. We see fences, arches, and bridges, such as the Quebec Bridge which counted Gustaf Eiffel as one of its contributing consultants, a symbol of increased circulation, movement, and commerce. A plane flies across the building and it transforms into a ruled line of illuminated numbers: the building becomes a massive radio. According to Lepage (2011), we hear "opinions, ideas, expressing the idea that Quebec is becoming a modern city." The exposition uses a great deal of repetition and pattern, mostly because it is impossible to take in the entire screen at once unless one is up on the ramparts of the city or in a hotel room quite some distance away. There is no privileged viewing position: if one is up close one might experience the bass of the subwoofers under the dock, but will not be able to see the entire panorama. If one is far away, one will have to contend with a less impressive radio broadcast of the audio but be able to see the entire projection situated amongst the rest of the city. What unites the viewers in the end is the city below their feet and the massive image that recenters their bodies and eyes.

The images speed up, mimicking revolutions in technology and culture: a library shelf is filled, x-rays are performed, and "tiller girls" (Kracauer 1995) grace the cabarets. The silos become a huge piano, with a total of eight hands dancing across the keys, playing a 
single tune. A public demonstration takes over the building, which morphs into a hockey fight, which becomes a large highway traffic sign, then a stock ticker, and a heart rate monitor indicating the beating heart of the city disrupted by a traumatic event, the shooting that took place at the Quebec National Assembly in 1984. The city is united in a symbolic death and rebirth. It then becomes a huge screen for a video game, a nod to the strength of the special effects and tech industry in Quebec, then graffiti. As the story winds down, we see images referencing the new airport and cruise ship terminal in the city, part of a further opening up and circulation of people and culture in the city. An extra-diegetic searchlight turns on atop the projection and real smoke emerges from the building while the image of an engine liner floats below. Fireworks are projected, a nod to the kind of light shows that usually commemorate such civic anniversaries. Next, a cheering crowd appears like one would expect at a theatre or outdoor concert, reflecting the city in that very moment back to itself. This image recedes, a vault is closed, and the building fades not to black but back to itself, lit modestly and appearing once again like a sleeping giant.

In the following sections, I describe the ways The Image Mill harnesses ambivalent and contingent relational space afforded by public projection and illustrates aspects of a "new monumentality" and the public sphere through three specific characteristics of cinematic media: superimposition, montage, and dispositif/apparatus.

\section{Superimposition: Super Imposing}

"The pure art of cinema exists almost exclusively in the use of superimposition"

- Youngblood (1970, 87) 
According to Gunning (2007), superimposition is the "incongruous juxtaposition" of images in film that "... yields an eerie image of the encounter of two ontologically separate worlds" (6). In Hitchcock's work, for example, this might serve the purpose of revealing some internal state, an aspect of memory, or connect the action to another space or time. Of course, these superimpositions occur within the diegetic space in the cinema. The superimpositions in The Image Mill, and in architectural projections in general, enact these same possibilities while extending the potential for ontological hybridity and narrative and creative expansion across diegetic and non-diegetic space.

The Image Mill used visual, tactile, and sonic superimpositions to open up new narrative and associative potentials that serve to rehistoricize its location and create affective responses from its urban audience. Sounds emanating from speakers in a dedicated "amplified area" (see Figure 10) between the Old Port Market, Dalhousie Street, and Quay St-André Street across from the silos linked a certain quality of sound to a place (the sounds from within the silos, the nostalgic songs that were once played on the radio), and "The Mill Frequency 97.5" accommodated radio listeners within the viewing radius created by the projection and the stadium-like setting of the city. The producers of The Image Mill added another dimension, tactility, in the form of the subwoofer vibrations that shook the dock across from the silos where many spectators congregated for the show. As horses raced across the projection surface, then trains, and later automobiles, viewers could feel the change of pace in culture created by the vibrations of the viewing area and imagine a time when these vibrations were not simulation but reality. Coordinated visual, tactile, and spatial superimpositions in The Image Mill like this were specifically designed to create 
a bridge between history, space, and memory. The ships projected onto the massive grain silos also matched the scale of the actual ships in the harbour, creating moments of misrecognition that tied two times to one place. Towards the end of the show, a searchlight emanated from the top of the projection and real smoke emerged from the building while the image of an engine liner floated below, again, inching asymptotically closer to a unity of space and image. Finally, at the close of the presentation, a cheering crowd appeared like one would expect at a theatre or outdoor concert creating a mirror effect, reflecting the city and the importance of the presence of the audience in that very moment back to themselves.

With this expanded sense of superimposition, the public monumental projection of The Image Mill created an encounter between "ontologically separate worlds," (Gunning $2007,2)$ that of the expansive moving image space, the cinema, and the city, thus mixing the times, spaces, characteristics, and inhabitants of all three. As Gunning points out, "The ontological argument claims that photography not only portrays things but participates in, shares, or appropriates the very ontology of the things it portrays." While for Gunning this ontological bridge might mean that the phantasms that grace a screen in fact infect it in some way with their spirit, with The Image Mill the converse may be true: the building itself is filled with spirits that emerge in the diegesis. In this way, the surroundings enter into a relationship with the images, sharing and extending this hybrid ontology.

The superimpositions of The Image Mill created an ontological bridge that united people, space, and image in a collective performance of memory. As noted on the official website for the project, the building was meant to be "in a constant state of transformation, 
giving viewers the surreal impression that it is alive and talking to them" (EX MACHINA 2014). This was partially achieved through superimposition. Likewise, Philippe Dubé, codeveloper of the original scenario for The Image Mill, points out, "Each evening the mass of reinforced concrete would turn into the segmented body of a giant beast to act out the story it had witnessed" (182). Superimposition was harnessed and extended into public space in The Image Mill, making the space more relational and furnishing new experiences of monumentality.

The city thus becomes cinema-that is, it becomes theatrical-and the "cinema" expands to become enmeshed in the urban fabric (Brougher 2008). As McQuire (2008) notes, the relational space that the moving image in public space creates narrows the perceptual and ontological gap between cinema and the city, between mediation and the built environment. The Image Mill uses superimpositions to create moments of mediainduced misrecognition where, as Gunning notes, "the criterion that separates real objects from their equally life-like apparitions" (21) is diminished. When the grain silos become a gigantic radio from the 50s, they, and the city, become possessed with a "liveness" and performativity: they share the same frequency and wavelength-they occupy a contemporary and historical resonance. The contingency of the moving image presented in this way, superimposed as it is on a wide expanse of urban space, expands the possibilities for a "new monumentality," just as Giedion and others predicted, creating temporary conditions for the condensation of meaning and collectivity where "a unity of the architectural background, the people and the symbols conveyed by the spectacles" (Giedion 1944, 568) arise. 


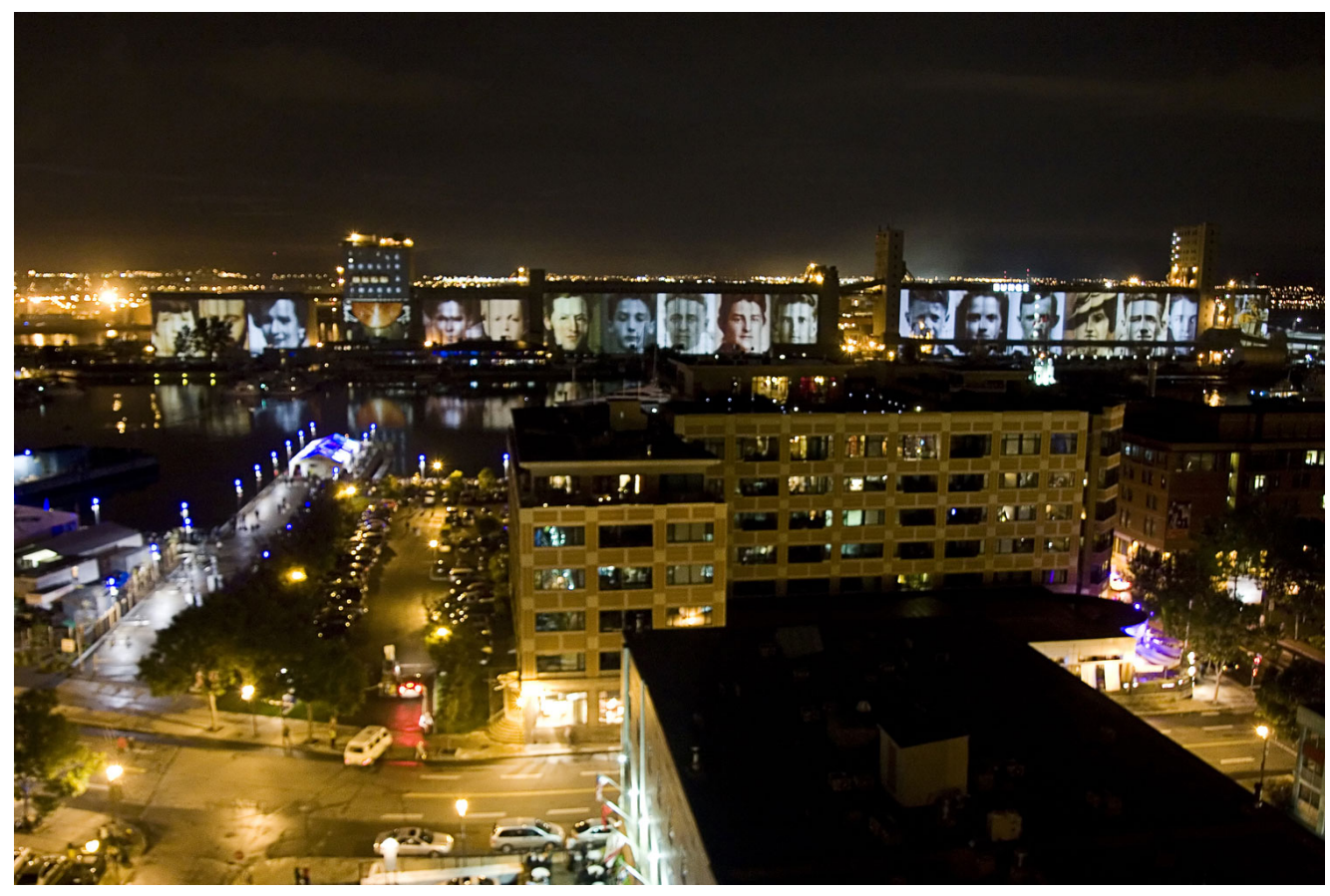

Figure 9. Portraits of Quebecers from The Image Mill on the façade of the BUNGE, Quebec City.

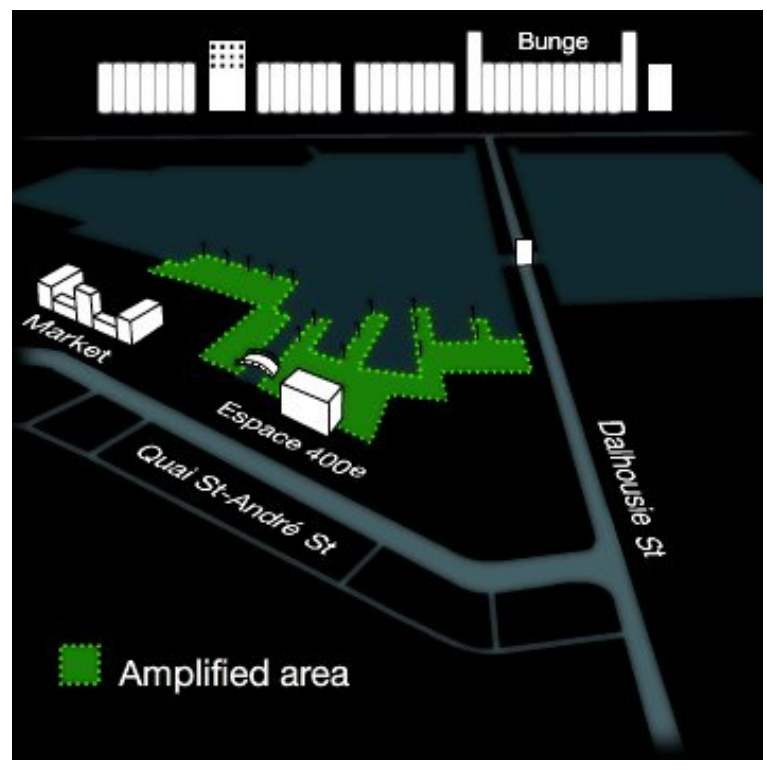

Figure 10. The "Amplified Area" of The Image Mill. Source: lacaserne.net. 


\section{Spatial Montage: Extra Diegetic}

Montage also plays an important role in extending cinematic space in the massive media of The Image Mill and provides an expanded means for associative and narrative capabilities. Typically, montage in film is achieved in editing by juxtaposing different shots with one another, cutting and recombining film in order to create relationships between images from different times and/or spaces. The practice of montage has expanded over time to include the additional use of composite montage within frames, itself a variant of superimposition, that has accelerated greatly with digital editing techniques. Still, through cutting and recombining film or altering layers within the digital frame, montage remains primarily diegetic. Rarely, if ever, does the montage explicitly reference or interact with its frame and that which lies beyond it, where the frame is assumed to be the neutral black edges of a darkened theatre, television set, or more commonly now, a mobile device.

The nature of montage changes when the moving image leaves standardized formats of presentation, abandoning their present-day interoperability and remediationthe any place, any time, any where-ness of contemporary media (André Gaudreault, August 5, 2011, e-mail to author)-for a singular specificity precisely located on a unique surface within the shared frame of an architectural or urban space: a massive media event. When montage occurs in public space, the logic of the monument and the logic of the cinema are combined to expand the dimensions of a site thus fostering greater narrative and associative flexibility between moving images, architecture, and people.

Spatial montage in massive media juxtaposes the moving image of the spectacle with other moving images around it, with the city, and with other fragmented media forms 
(such as radio frequencies, subwoofer vibrations, or social media). The Image Mill was developed specifically to engage in a spatial montage with its setting, its multiple media elements, and through its use of scale. For one, the diegetic montage cycled through a set of scales, from magnifying the microscopic to the scale of the building, to compressing the cosmos onto its surface, or by displaying scenes of buildings or cathedrals mapped onto the building at roughly the same scale as they might actually appear. While cinema traditionally does this in the space of the theatre designed to be isolated and sheltered from the conventional world outside it, The Image Mill engages directly with this world, imparting an oneiric quality to it through a montage that directly references and interacts with this world. Viewers could see the ramparts appear on screen before them and turn around to see the very same structures at roughly the same scale surrounding them. Spatial montage infects space to prompt meaningful and instructive comparisons with it-it sutures space and cinema.

Spatial montage also extends what Kuleshov calls "creative geography" (Kracauer $1965,48)$ to public space, thus contributing to the production of a space made relational by way of media. Creative geography primarily refers to the illusion of spatial continuity created when material phenomena captured in different places are juxtaposed in cinematic montage: actors exit a house on set and emerge in an "on location" street scene; an aerial shot depicts a certain mountain village and we cut to a scene in a different place altogether while believing it to be in that village. The Image Mill engages in "creative geography" throughout its entire presentation by virtue of its situatedness in the urban environment: as the viewer looks to the screened images, then to their surroundings, and back again, the 
urban space is continually compared to the images designed to reference it, appealing to an extra-diegetic congruity. Every image it displays is implicitly related to its surroundings and asks to be either merged with it or to resonate within it. This is particularly apparent when the silos transform into various architectural elements that can be found in the city, or could have been found in the city, throughout history: ramparts, stained glass windows, office blocks, and the log fences of early settlements. These represent, in their congruous architectural scale and extension, a merging of cinematic and geographic urban space. When the building becomes a giant scoreboard for a hockey game or the LED signage used to direct highway traffic, a spatial montage is created through the inflated scale afforded by the massive projection that creatively transforms the entire city into an arena or a highway. Thus, The Image Mill uses images that refer to and employ the space around it to engage in a kind of creative geography that Kuleshov could not have considered at the time, creating illusions of both spatial and temporal continuity and reinforcing ontological bridges created through superimposition.

Both spatial, extra-diagetic, and mediatic superimposition and montage represent an ontological blurring between diegesis and surroundings and a new kind of creative geography. Spatial montage and superimposition contribute to the framelessness and spatial ambivalence of the massive media experience expanding the field of representation and the possibilities for narrative and association between the city, people, and the moving image. 


\section{Dispositif and Apparatus: Staging the City}

Apart from superimposition and montage, massive media also exhibits an expanded and experimental sense of dispositif and apparatus which shows it to be integral to the development of relational space and the renovation of the public sphere and monumentality in public spaces.

Albera and Tortjada (2010) offer the following definition of dispositif: "It may designate any type of technical organisation or construction, or any arrangement, including with human actors, as long as it correlates actantial positions and relations" (10). Thus, it is a situation in which human and non-human actors are placed in prescribed relations with one another through socio-technical means. This definition draws upon Foucault's definition of the term, often imprecisely translated as "apparatus." Foucault (1980) defines dispositif as "a thoroughly heterogeneous ensemble consisting of discourses, institutions, architectural forms, regulatory decisions, laws, administrative measures, scientific statements, philosophical, moral and philanthropic propositions-in short, the said as much as the unsaid" (194). Thus dispositif sees power embedded in socio-spatial ensembles that place actors, living and dead, into specific relations and direct them to experience themselves and the space they occupy in prescribed ways. Examples might include the classroom (with dispositif elements ranging from its regulatory board, forbidden language, the blackboard, the instructor, the students), or even the prison (with elements such as the cell, behavioural protocols, uniforms, surveillance systems, laws, courts, and so on). In cinema, dispositif is "a network of relations between a spectator, the representation and the 'machinery' that allows the spectator to have access to the representation" (Albera and 
Tortjada, 12). At the same time, the dispositif of cinema also allows the representation to have access to the spectator: dispositif is the ideological interface that captures and captivates, delivering an audience to a message. Apparatus, on the other hand, is a term better used to refer to the technical aspects of this relational field of actors, or dispositif. Apparatus is the concrete existence of dispositif, while dispositif is the discursive existence that includes these concrete elements. For cinema, the apparatus includes the screen, the projector, the film, the seats, and so on. As Jean-Louis Comolli (1980) argues, cinema in fact did not really exist until the incorporation of the fledgling apparatus of cinema into a larger dispositif, that is, the incorporation of technical means into an ideological program. As he notes, "A dispositif was required which implicates its motivations, which be the arrangement of demands, desires, fantasies, speculations (in the two senses of commerce and the imaginary): an arrangement which gives apparatus and techniques a social status and function" (122). Thus, the apparatus of cinema can be directed towards forwarding imperialist, capitalist, sexist-or counter-imperialist, counter-capitalist, and counter-sexistideologies.

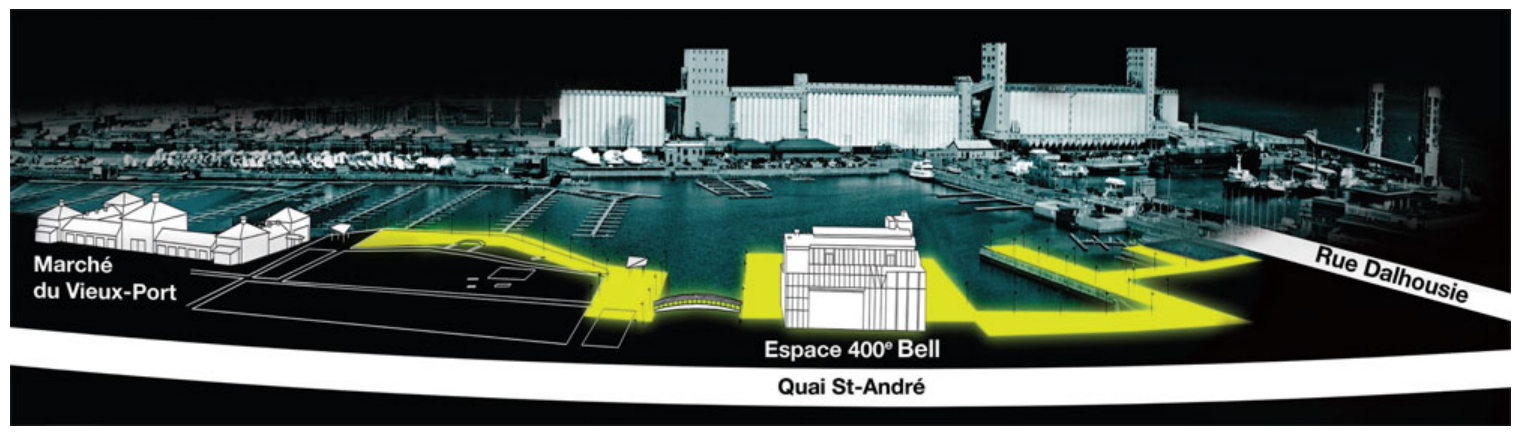

Figure 11. Ideal viewing positions for The Image Mill. Source: lacaserne.net. 
For massive media, the apparatus, which includes elements of the city and the application of various media, is less clearly defined than the cinema yet together they form the basis for the arrangement of demands, desires, fantasies, and speculations for citizens and the city alike. As a result, The Image Mill can be seen as a composite dispositif (Verhoeff 2012) of an open-air theatre, the cinema, and a broadcast model like radio, that is, a state of mediation in which standardized environments of spectatorship, their affordances and attendant ideologies, overlap and co-constitute a relational experience. The Image Mill extended and combined the efficacy of these elements of transmission borrowed from previous forms by design but also at times out of necessity. For example, The Image Mill did not create a darkened cinematic space to focus attention on the diegesis so much as it had to wait, patiently, every night, for the sun to disappear on the horizon so that an image might appear on the silos. In this way, an incomplete dampening of sensory stimuli from the surrounding space was predicated on a celestially controlled shift from the diurnal to the nocturnal, thus ceding to this natural rhythm. As much as the creators hoped to "turn the city into an auditorium," (Lepage 2011) they could only do so by first deferring to predictable but unchangeable forces of nature. The show began at 10:15pm in June and July, and at 9:45 in August and September. Commenting on this natural phenomenon, production designer Mario Brien (February 9, 2014, phone call with the author) noted that "It was dark of course, but not dark enough to be satisfying at times-some nights if the moon is full, the clouds are low, and city light reflects on the clouds, light spills on to the screen." To compensate for this, some control over lighting was exercised by the production team as they were granted access to the street lighting infrastructure along Quai 
St-André across from the silos (see Figure 11). Although their efforts did not necessarily make for a brighter image on the screen, it did create a cinema-like pocket of collective darkness for spectators, thus borrowing this device from cinematic technique. It also showed the dispositif of massive media to necessarily be one that was a composite of natural and imposed conditions.

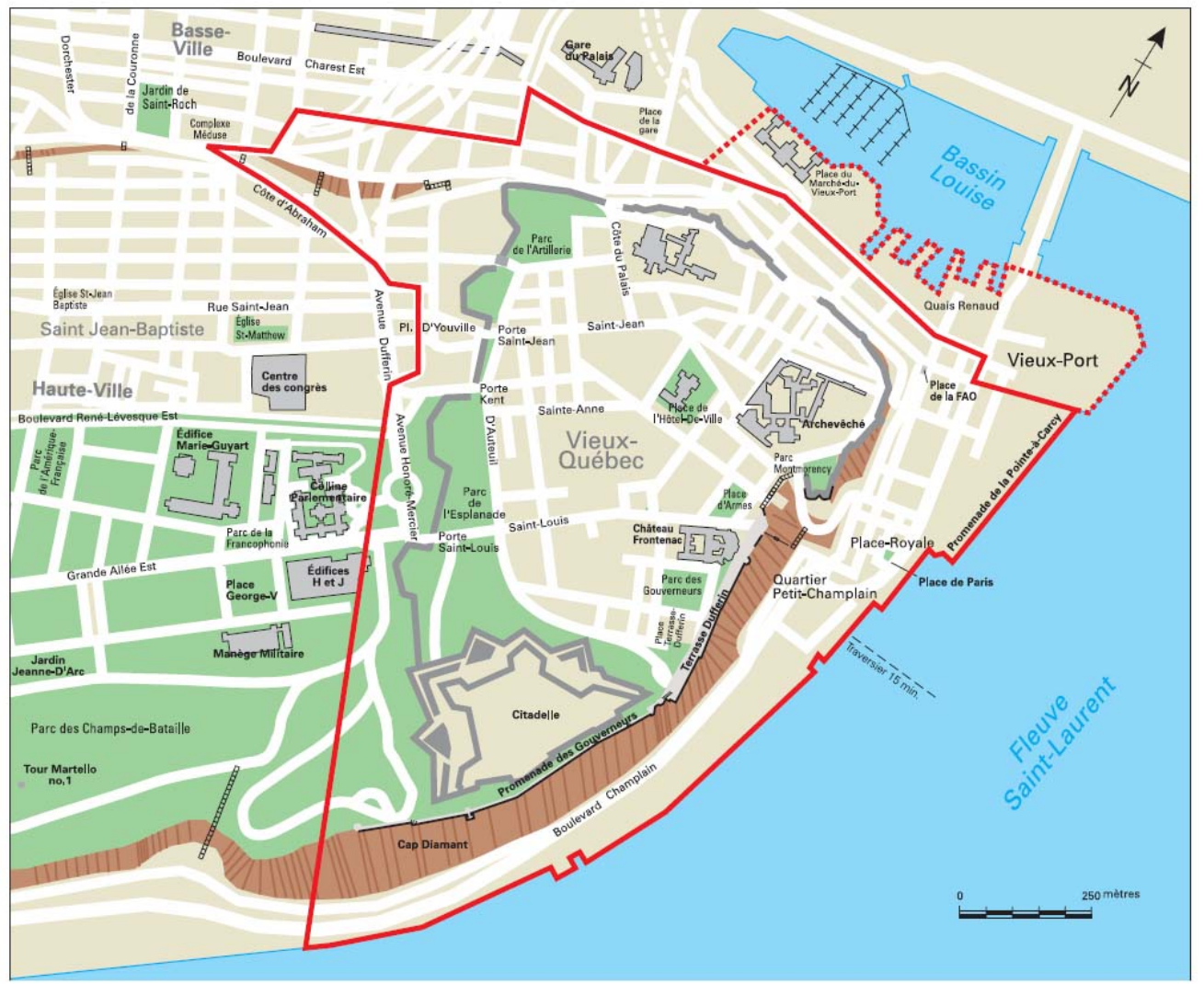

Figure 12. Viewing area for The Image Mill. Source: UNESCO.

Audience recentering and diegesis delivery occurred through extra-diegetic composite dispositif elements that engaged sound and touch as well. "The Mill Frequency," a radio signal that carried audio for "those who want[ed] to experience the mega-projection 
from afar" (EX MACHINA 2014) reinforced the communal aspect of the presentation and contributed to a democratic surround where pieces of information related to the presentation were made accessible to variable recombination by a wide audience. The provision of a radio soundtrack effectively extended the audio/visual apparatus of the presentation so that it was limited only by sightlines which, aided by the natural elevation to the south of the projection surface, extended approximately one kilometre away (see Figure 12). Mario Brien (February 9, 2014, phone call with the author) described the upper town area as "a balcony, the same as a theatre" and noted that the scale and quality of the show meant that "The relationship between the public and the stage was almost the same as in the theatre" despite the usual urban distractors (traffic, pedestrians, sounds). Similarly, as Demers (2010) notes, the "monumental building also allows you to watch the work from several different points, using the spaces of the city as bleachers" (48), downplaying the idea of a privileged viewing position emphasized by the dispositif of cinema. By mimicking the conventions of spectatorship from theatre, film, and even sporting events, as well as providing non-localized and localized sound, The Image Mill created a multi-modal net of capture to re-centre a distributed, mobile, and diverse audience. 


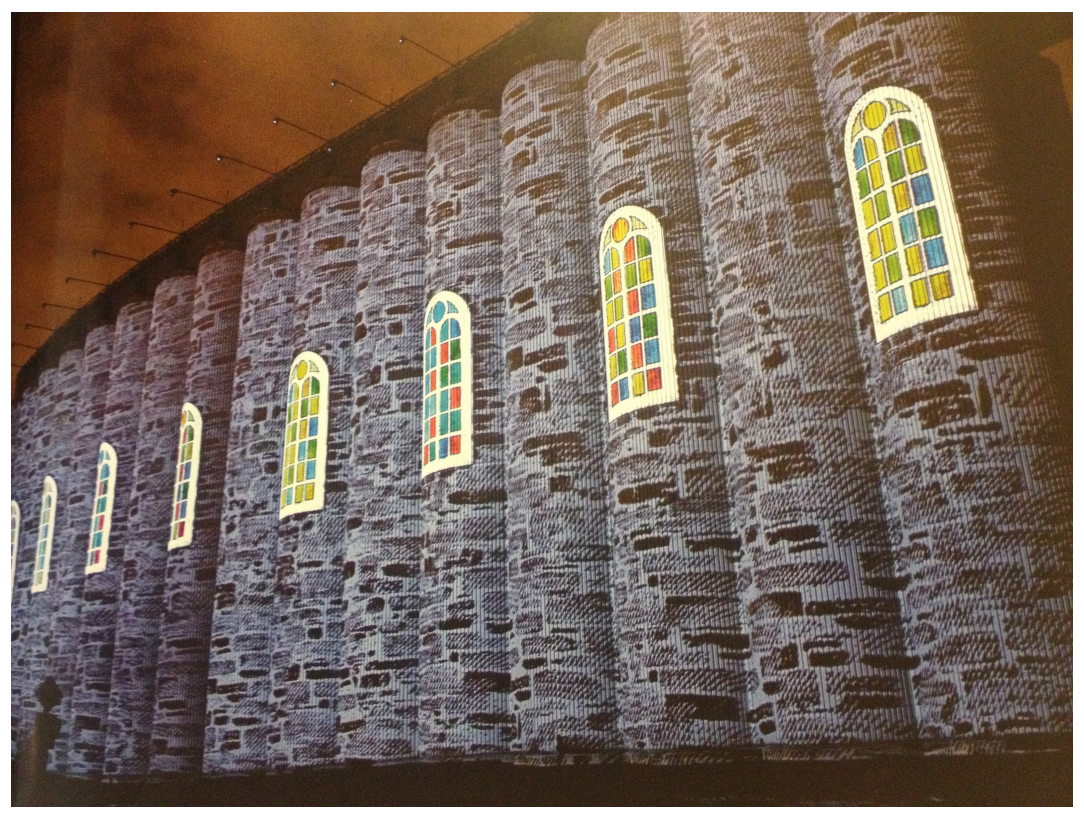

Figure 13. Stone walls with stained glass projected at scale. Photo: Nicolas Ruel.

Finally, an essential ingredient of the effect of dispositif carried forward and amplified in monumental projection of The Image Mill is the role that scale plays in determining relations of power, meaning, and attention in scenarios of spectatorship. Doane (2009) argues that expanded cinema resuscitates the body as a measure of scale and distance in physical space that has been lost to a degree in the practice of cinematic spectatorship, a practice that seeks to erase the spectators proprioception and deliver them to some aspect of the diegesis, whether that be an identification with a subject in a film or with the camera. If monuments, for example, transfer power with their surroundings, massive media allows for this transfer to occur in a number of directions: between monument, body, image, and environment. Scale in massive media serves to help unify the presentation with its surroundings, recenter an audience, and create experiences that heighten, not diminish, the embodied experience of the spectators and their ability to see 
themselves as the centre of the experience. In the case of The Image Mill, scale puts the spectators body in a direct spatial relationship to historical images amidst historical objects: it merges the archival and memorial qualities of space and media creating an embodied experience of history and transforming the viewer into a vector of inclusion and potentiality. The expanded proscenium of the monumental projection demands that the screen be considered in relation to its surroundings within which the spectator is treated as a moving focal point.

\section{McLarena: Recentering the Audience}

In addition to scale and site-specific interventions that cater to a mobile audience, the elements of direct participation and interaction add yet another recentering device to the dispositif of massive media. These elements, afforded by tangible interfaces at the site or mobile ubiquitous media, present additional aspects of a composite dispositif of media and monumentality that help to capture and deliver audiences to new experiences and rituals of collective memory.

Perhaps the most consistent site for this kind of experimentation in the last decade has been the Quartiers des spectacles in Montreal. Since 2003, the Quartiers des spectacles, a coordinated area of public spaces, lighting, and permanent architectural projection sites, has been at the forefront of developing interactive cinematic experiences in public space at an architectural scale. A recent example, McLarena (2014), at the Saint-Laurent Metro Station site within the Quartiers, demonstrates new ways in which the city, the moving image, and architecture can be combined along with interactive digital capture techniques 
that place the body in direct relation with historical material and other spectators in public space (see Figure 14). While building upon traditional means for capturing attention and generating affect and meaning in the cinema (superimposition, montage, and dispositif), McLarena adds to the composite dispositif of public projection by including a powerful suturing device of direct involvement through interactivity.

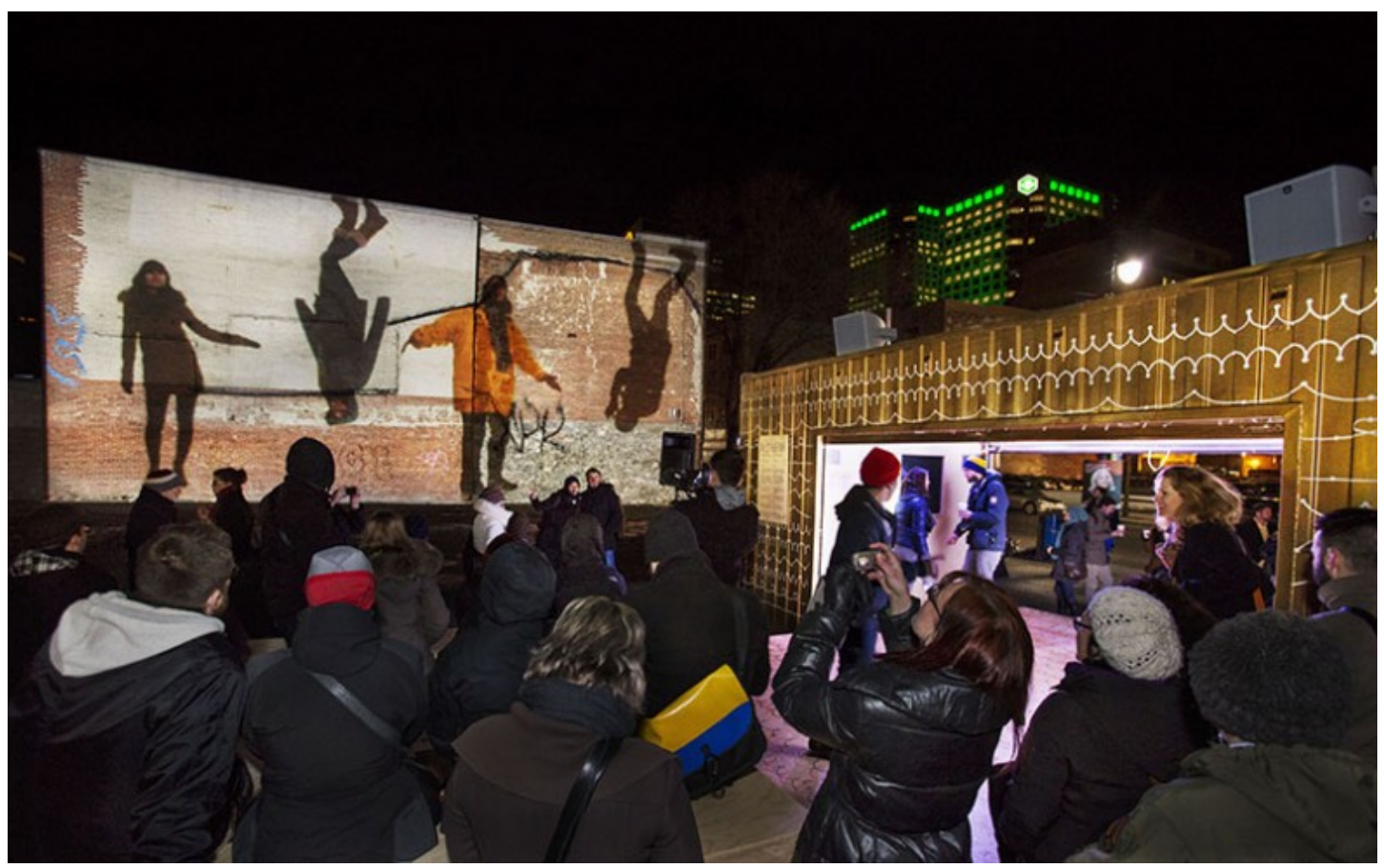

Figure 14. Daily tous les jours, McLarena (2014), Quartiers des spectacles, Montréal.

The creators of the work, Montreal-based design studio Daily tous les jours, describe McLarena (2014) as:

... a playful participatory work that invites passers-by to imitate the choreography of the character in McLaren's film Canon (1964), which depicts the musical genre of the canon in animated images. For McLarena, a video booth has been set up in a shipping container outside the Saint-Laurent metro station. Each participant goes into the booth and is filmed on video while 
attempting to perform dance steps based on the preceding participant's video. The results-errors, transformations, and an evolution of the movements-are all captured in an endless video created by the participants and projected on the façade of an adjacent building. (Quartier des spectacles 2014)

McLarena asks viewers to watch and mimic parts of Canadian film pioneer Norman McLaren's original film Canon (1964) while their image is captured and automatically processed and presented on the architectural façade situated outside of the Saint-Laurent metro station. McLarena also includes a tiered seating space next to the mobile video production booth so that people can watch the performance of the participants as they attempt to mimic McLaren's work as well as the projection that displays the results of their efforts.

Canon depicts a single dancer occupying only a small portion of the frame. The dancer proceeds from left to right, twirling, tipping his hat, kicking the air, snickering and stumbling to the side, ducking, then proceeding off screen. The single dancer enters again, but this time is followed shortly by his double, and then a double of that, and so on, until we begin to see not a pas de deux, but a pas de trois, then quatres, as dancers are recycled on the left of the screen just as they exit on the right. All the while, a musical motif is repeated in the background and overlapped with itself at different intervals, just as the animations are, typifying the theme of the musical canon where the same melody is introduced in different parts so that the imitations overlap in pleasing ways. The relative simplicity of its visuals and choreography, the concept of overlap or superimposition, and the deep connections between Norman McLaren and the NFB to Montreal, meant that Canon was 
historically, stylistically, and conceptually amenable to a reinterpretation through a participatory outdoor projection.

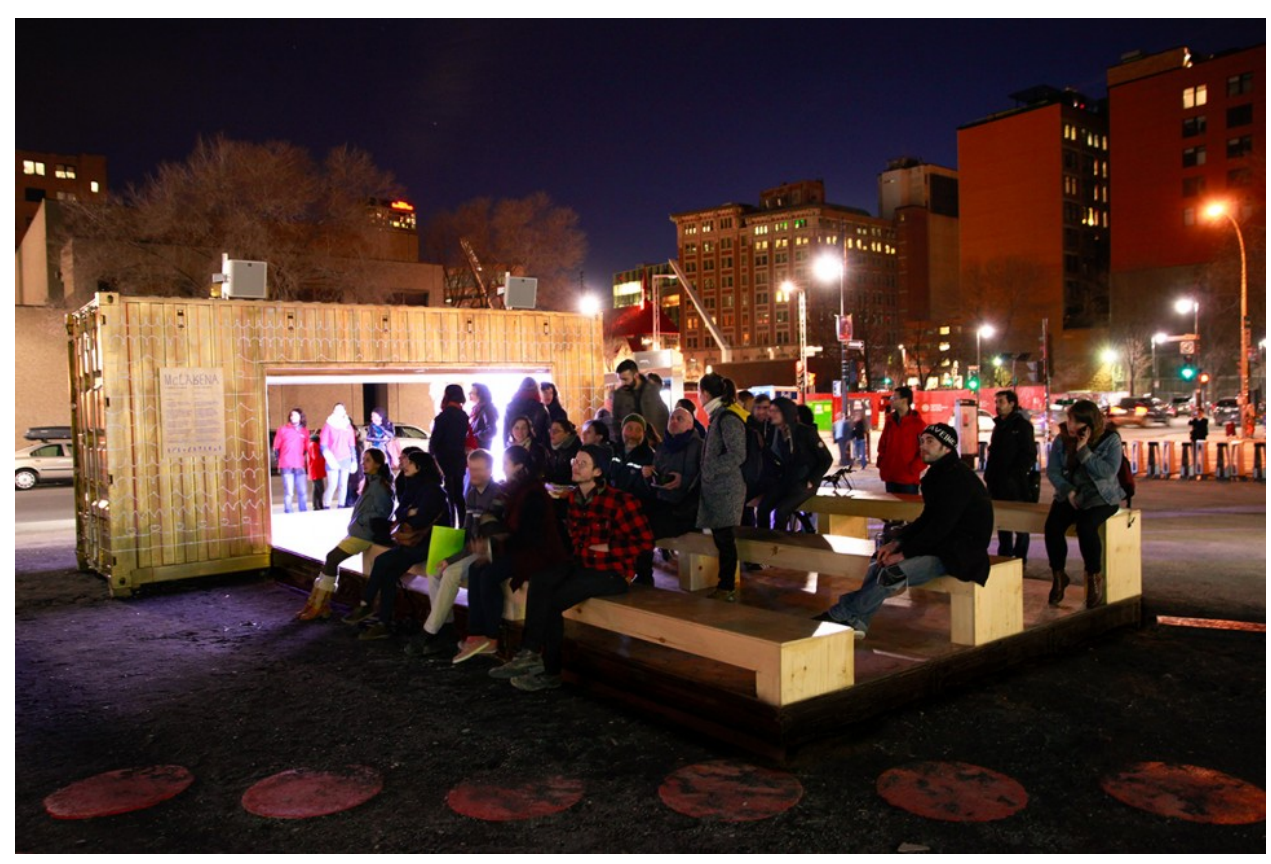

Figure 15. Interaction space for McLarena (2014).

McLarena presented an interesting mix of cinematic techniques repurposed for monumental projection: the literal superimposition of the participant in the diegesis, the superimposition of the larger image within the cityscape, a spatial montage that puts this installation into a relation with the surrounding architecture and flows of pedestrians and cars, and the traditional viewing conditions of seating (i.e., tiered) augmented by an expanded scale and the addition of an interaction space (see Figure 15) that sutures the interactant to the image. McLaren's film was simultaneously viewed by participants in the mobile recording unit, learned, and remediated through their bodies, then processed, amplified, and represented in the very style of McLaren's film on the side of a building. 
Each of these elements served to deliver the various actants (viewers, participants, and passersby) through a composite dispositif that crucially included direct participation. It connected a publicly funded organization and archive (the NFB) to the publicly funded Quartiers des spectacles, thus creating a new public for ludic encounters in a totalizing mediaspace. A unity was inscribed between a collective, a history of media, and a place through participation in a massive moving image.

\section{Participation: Don't Just Sit There and Watch}

While McLarena is ostensibly participatory, it is important to consider the quality of the participation it engendered as a work of massive media and the qualities of a public sphere it enacted. Did it contribute or support meaningful interaction that is unique to the form? Is it an artistic equivalent of a productive political position? Is it an extension of the concept of the democratic surround? Claire Bishop is critical of the quality and meaning of the modes of public participation afforded by certain assemblages of expressive displays, digital sensors, and networked devices. In her discussion of relational aesthetics and the idea of activated spectatorship in installation art, Bishop notes "the public sphere remains democratic insofar as its naturalized exclusions are taken into account and made open to contestation" (65). In the context of public space, these "naturalized exclusions" include the exclusions of sociability enforced by the prioritizing of flow through spaces, particularly as it is enacted outside of a subway station, for example, as well as the prohibitions related to the rights of the citizen to alter their surroundings or see themselves reflected back in it. While McLarena does not necessarily promote direct discourse about the history of 
Canadian film, arts funding, or other issues that might be associated with the installation (although it certainly does not downplay these either), the inclusion of participation at an architectural scale in public space can be seen to address the naturalized exclusions of public space itself, seeking to arrest the spectator, and provide a scenario in which sociability is fostered and reflected at an architectural scale.

For Bishop, what is central to authentic participation in art is "activated spectatorship" $(2006,50)$, or a scenario that values equality over quality, collective authorship over singular control, and the ongoing struggle to find artistic equivalents of political positions $(2012,3)$. McLarena certainly embodied these tenets in a number of ways and the resulting film or video was by no means a quality reproduction of McLaren's original (and this is part of the contingency that makes it attractive). The artistic scenario it created was one that elevated and amplified individual identities while encouraging sociability through the recentering devices of scale, seating, setting, and participation itself. While, again, McLarena did not explicitly address politics, it did symbolize a political position, one that combats the privatization, atomization, acceleration, and flow privileged in public spaces, the inaccessibility of building scale effects by everyday citizens, and the calcification or sedimentation of history. It critiques spectacle by reproducing it and subverting its logic for alternative ends. That it did so in a way that encouraged public sociability and an active participation in history is also a testament to the larger designs of the Quartier des spectacles to create temporary publics and ludic interludes that foster the bending and breaking of proscribed social protocols in the city. McLarena and the Quartier des spectacles can be seen to be striving for a new democratic surround, one in which 
democracy is extended through access to building scale affects that encourage citizens to self-represent through government supported and sanctioned channels of expression that carry with them a message that is transferred in and through a composite dispositif of media and urban space.

\section{Place Branding and Theatricality}

While McLarena may be seen to have provided a means to create new associative experiences and rituals with media, the memorial, and the monumental, as well as having provided a critique of spectacle as it relates to public space, it can be seen to have been captured by spectacle at a larger scale, that is, at the level of place branding and entertainment that have become economic imperatives for cities competing at a global scale. Susan Bennett (2009) specifically argues that the extension of the cinematic and theatrical to the city and passerby via large-scale public projection events as evidenced by The Image Mill, and, I would add, by McLarena, represents a key tactic for differentiation and status within a globalized tourism industry. McLarena certainly contributed to the wider socio-economic goals of changing the image of public space in Montreal and in the Quartier des spectacles in particular, an area once permeated by seedy bars and strip clubs. This was achieved, according to Bennett, through a "mobilization of theatricality" (9) which refers to the way works such as The Image Mill and McLarena encourage people to see space as fundamentally theatrical and thus as venues for entertainment. The Image Mill did this to the whole of Quebec City by presenting a work that superimposed a cinema or theatre onto the city's surfaces, while embracing and supporting multiple, incomplete 
combinations of media forms, or a fragmented or distributed audience-what Bennett describes as "peripatetic" (12)-gathering these disparate groups together first and foremost in the illumination of the space with the moving image. The same can be said of McLarena, which made a similar superimposition into public space, creating a theatre with sound, seats, and screen, while taking the peripatetic aspect of The Image Mill and expanding it through the movements that are encouraged, captured, and remediated into public space by participation enabled by the mobile recording unit. The result, in both cases, was the infusion of a "liveness" (12) into public space that made it performative and theatrical, and made it useful for the purposes of coding a space as open, playful, progressive, and inclusive. In other words, the experience was seen as valuable in the eyes of the city, many of its citizens, and developers. As such, through these composite dispositives of massive media projection, media archives were mined, processed, animated, and delivered to audiences (as audiences were delivered to them) to enliven and entwine the archive, the space, the spectator, and the city, as well as to bolster economies of tourism and migration.

\section{A New (New) Monumentality?}

The coupling of the digital, the moving image, architecture, archives, and public space present unique challenges for the arts and public culture, providing the potential for new democratic and historical registers of monumentality. It also threatens to dehistoricize, commercialize, or aestheticize public space and the public sphere. While massive media furnishes new opportunities to shape collective experience, it also, as Joel McKim (2012) argues of the Quartiers des spectacles, does not guarantee artistic innovation and can just as 
easily contribute instead to the "general aestheticization" (135) and branding of the city for touristic and economic purposes. This concern was perhaps most clearly demonstrated in the Nokia example discussed earlier in this chapter but still exists, to a degree, for all massive media works.

While the realization of the self both in and against the image and interaction space in public space has democratic and progressive implications for the future of public sociability, there remain deeper political questions regarding the commodification of cities and public space as well as the exploitation of the attractive potential of participatory and theatrical public spheres. Although not overtly commercial ventures, the crowds that assemble for events like The Image Mill and McLarena invariably become part of the symbolic capital assets of the city and thus enter into the wider scale of civic economics. In this way, cities like Montreal run the risk of becoming addicted to developing and maintaining a "symbolic primacy" (76) through large outdoor installations that seek first and foremost to demonstrate their global identity rather than carefully considering how local economies, histories, and residents may be affected. A general concern of massive media works is that, given the outlay of funds required to support them, tourist revenues will skew artistic autonomy and diminish the quality of works, or that art, culture, and the public sphere will be further commodified through massive media. Signalling this, Mayor of Quebec City Regis Labeaume notes a trend in tourism that he calls a return to "the era of the city" in which the key metric for differentiation between cities is "culture." Labeaume highlights the strategic importance of cultural activities within the highly competitive world of cities to attract tourist dollars, skilled workers, and economic 
investment. Similarly, comparing Tunis to Quebec City, Saidi (2012) notes that "heritigisation and touristificaton policies in these two cities, especially policies that lead to their museumification through the promotion of practices and aesthetic values transform them into open-air museums" (75). To that end, the government support of The Image Mill, a $\$ 4.3$ million dollar a year project (“La Ville..." 2013), is a clear investment in developing a tourist clientele for the city and reinforcing the "symbolic capital asset" (75) of the city. As in the cinema, the crowd is a semiotic and economic resource regardless of the reason for its gathering. Despite this, the sites analysed in this chapter in Montreal and Quebec City, while susceptible to commercialization and aestheticization, do transcend these challenges to a degree in both their experimental superimpositions of the theatre and cinema onto the city, and their content that attempts to reflect and refract citizens and their shared history through architectural space and interactivity, thus creating new practices for ritualized public sociability and political positions in public space more closely associated with democracy than their crassly commercial counterparts.

Perhaps more importantly, the provision of permanent or semi-permanent projection sites as found in The Image Mill and McLarena-something that lies beyond the means of individual artists, curators, or citizens-allows for greater syncretism and experimentation with new rituals of monumentality that make these sites more legible and habituate audiences to new modes of reception, allowing the sites to exist beyond frivolous spectacle, advertisement (civic or otherwise), or decoration. The coordination, curation, and long-term planning for these sites is of utmost importance (something that is addressed in detail in Chapter 4) to invoke new, meaningful connections with massive media in 
public space. In this way, large-scale projection sites can represent a new "new monumentality" (Mumford 2000, 151) when artists are allowed to develop audiences, spaces, and images that attempt to reflect and renovate the social and communal life of a place with elements that borrow from practices of public space, monumentality, cinema, and digitality.

As noted earlier, it was Giedion, writing in the mid-twentieth century, that argued for a revolution in monumentality, a new monumentality that would see monuments become better unified with their architectural background and where people would play a key role in the emotional events centred upon them. New experiments in massive media can represent an extension of these ideals, producing new audiences and spaces with new rituals and habits of identity and place formation that transform Giedion's tenets in a digital age in need of tangible collectivity. Massive monumental projections, particularly those that involve collective interaction actualized on an architectural scale signal a new dimension of networked media saturation and superimposition that captures the attention, actions, and image of a diverse audience and merges it with the cityscape in unique ways that make memory and history affective, embodied, collective, and temporarily concrete.

Equally important to this new monumentality is the concept of "post-production," or the documentation, dissemination, and archiving that occurs officially and unofficially before, during, and after the production of massive media such as The Image Mill and McLarena. In fact, Hito Steyerl argues that "post-production" is the true mode of contemporary image production. Presentations such as The Image Mill serve as important catalysts for this more important circulation. As Steyerl notes, "We are embedded within a 
post-cinema that has been completely transformed, mutated into whole environments, permeating reality to the point that we can now understand it with media thought and alter it via post-production" (quoted in Shental 2013). This certainly fits within the theories of the competitive city as museum as it envisions its tourist clientele as co-producers and copromoters of a city and seeks to cater to them through experiences situated within the city as stage or theatre. Of course, with the inclusion of interaction spaces, such as those found in certain outdoor projections in Montreal's Quartiers des spectacles, the city becomes more stage-like, more theatrical, and eminently more attractive and capable of post-production at and beyond the site and time of projection. That said, it is the communal character and scale of the massive media event upon which this economy is centred even as its traces are scattered long after the crowds have dispersed. Massive media are the necessary, temporary, substantiations or ventilation of media at the heart of this new cultural equation that involves people, media, memory, and space.

As another potential aspect of a new, new monumentality, the massive media works presented in this chapter can be said to perform a rehistoricization of space through the multiple impressions and transformations they engender. This is exactly what may be needed as space becomes more hybrid and relational: carefully constructed interfaces that incorporate the mutable digital image into shared spaces in order to enact powerful, yet heterogeneous, variable, and impermanent connections. As McQuire (2008) notes, these temporary substantiations better reflect the way we have come to interact with each other in space and through the moving image. While for some like Crary and Virilio this practice might represent the possibility of a dangerous spatial and historical amnesia by way of the 
trace of contingent media, exemplified by Freud's forgetfulness at the Piazza Colonna, others such as Wodiczko have shown how this can stain or mark a place so that an impression is made that lasts in memory (embodied or electronic). Digital projection in public space is a frontier for the colonization and/or democratization of affect and attention, and the renovation of communality and mass culture; a frontier that The Image Mill and McLarena explore for the purposes of commemoration and civic communication. Furthermore, the practice of massive media clearly understands and demonstrates that it is on the digital screen that our stories and identities are now being formed and presented and attempts to bring this to bear in more tangible, monumental places of congregation. While the contingency of surface effects produced by cinema, as outlined by Kracauer, engages affective responses, the digital image multiplies this contingency through its ability to be manipulated in real time, the flexibility afforded to projection locations and geometry by new digital projection technologies, and its amenability to personal appropriation and distribution through mobile ubiquitous media. As Bruno Lessard (2009) notes, “... Moulin's creators implicitly posit that it has become practically impossible to conceive of history without the moving image" (78). Certainly, they also mean to say that it is the digital moving image, in its ability to remediate archival images and sounds at an architectural scale, that creates a unique experience of and with history for spectators through superimposition and spatial montage. Similarly, it is clearly the digital dispositif that affords McLarena's playful relationship between the participant and architecture and engages memory as simultaneously embodied, performative, collective, and spatial. Finally, flexibility of the digital projection allows for experimentation, superimposition, and 
montage at architectural scales, bringing audiences into contact with archives, architecture, and themselves, in hybrid, relational space.

In particular, interactive massive media creates a monumentality that engages history (such as the history of Norman McLaren and the National Film Board) not explicitly through the historical, per se, but through a mixture of the historical and the perpetual present engaged by the digital capture and remediation of the very spectators it seeks to address, mixing the present reality of the crowd (on- and offline) with the civic and the historical, and the logic of digital media with that of the monument. In this way, works such as McLarena go further than The Image Mill in representing what Giedion speaks of as the "popular needs and aspirations" (Mumford 2000, 151) of our time, and thus represents in a truer sense a new, new monumentality. McLarena posits that not only is it impossible to "conceive of history without the moving image" (Lessard 2009, 78), but that it is impossible to conceive of history without seeing ourselves somehow included within it. Furthermore, history itself is now inextricably tied to digital information and thus must be made more open to remediation in and through public spaces, our historic sites of public contestation. Massive media provides a productive venue within which to explore and develop the tensions between history, technology, and public space.

Today, our needs and aspirations are a complex mixture of the desire to communicate in and through the digital image, to expect richer and more flexible interactions with our surroundings, as well as the overarching need for cities and citizens to see themselves within a desirable and globally relevant urban culture. As such, it is the lack of post-production facilitation that represents one of the critiques of The Image Mill with 
respect to its capacity to renovate monumentality. Lessard (2009) argues that the apparatus of The Image Mill does not go that far beyond that of the familiar positions of the television viewer or film spectator. He says that "the work's monumentality serves a very conservative historical content that is accompanied by an equally conventional viewing position, which lacks movement and reflexity" (79). What Lessard would like to see is a more challenging presentation, for the producers and the participants, one that incorporates networked elements, GPS mapping, or other reactive and algorithmic processes that reflect "a mobile notion of site and a nomadic subjectivity" (80). Lessard argues that the work lacks dimensionality that is demonstrated, I would argue, in something like McLarena, available to it by virtue of the digital tools that exist to incorporate real-time capture and processing.

That said, The Image Mill takes a giant step away from the presentation of a historical narrative within the confines of a museum or atop a static monument, and does allow for a degree of mobility and nomadic flows of communication and spectatorship, capturing something of the popular needs and aspirations of its audience and milieu, creating a relational space in the process. What the examples in this chapter maintain are the special effects of cinema, that of montage (albeit in a spatial form), superimposition, and dispositif (albeit in a composite form), which help to connect it to tried and true conventions of spectatorship and theatricality while disrupting public space sufficiently through the affordances of new digital technologies and productively troubling forms of mass culture, the public sphere, and monumentality. The city thus suffused by media enables a new communicative and commemorative environment that must be contested, 
developed, and claimed by artists lest it aestheticize and obscure history for the sole purpose of capital.

\section{Creation-as-Research: Experiments in Public Projection}

Issues of participation, intent, ownership, and history abound in the new complexities of space, monumentality, and the public sphere and these may be exposed in massive media installations. To further explore this situation I present a number of creation-as-research projects that enter into the space created by massive media to observe its affordances and limits directly. These projects provide examples and points of analysis of the application of cinematic elements and digitality in monumental public settings.

\section{0 moons many hands}

30 moons many hands $(2013)^{2}$ was completed with collaborator Patricio Davila. ${ }^{3} 30$ moons told the story of Douglas Carr, a shoe salesman from the small town of Ingersoll, Ontario, who spent thirty months travelling around the world in 1938, cycling from London to Cairo and through Africa to Cape Town. The installation featured a large-scale outdoor projection that displayed a montage of footage captured at the Archives of Ontario where his photographs, films, journals, and letters had been catalogued and preserved.

\footnotetext{
${ }^{2}$ See http://thirtymoons.ca/ for documentation of the work.

${ }^{3}$ Patricio Davila and I collaborated to create 30 moons many hands (2013). We were first introduced to the Archives of Ontario by Dr. Janine Marchessault at York University. Davila and I carried out research and documentation at the Archives of Ontario with the support of archivist Sean Smith. I was involved in storyboarding, video production, audio recording, web design, and initial video editing. Davila was responsible for video production, editing, and projection mapping. Both Davila and I were involved in the final production of the installation. We received in-kind assistance from graphic designer Patricia Davila (poster), photography assistance from Jamie Webster (documentation photos), and the in-kind donation of the use of two 10k projectors from York University's Sensorium: Centre for Digital Arts \& Technology.
} 
Davila and I initially met with archivist Sean Smith at the Archives of Ontario to discuss potential topics that we could present either on the building itself or on a building in a nearby courtyard. While Sean suggested a number of projects, he also gave us a tour of the facilities and the various labs involved in preserving and cataloguing material. In the end, we decided to tell two stories: the first was the story of cyclist Douglas Carr who cycled around the world in the 1930's in part because this material had just been donated and had yet to be used by other researchers or artists but also because of the richness of the imagery and text that in this particular fonds. Like McLarena, we realized that a key element in creating an engaging outdoor projection from historical material was to find existing audiovisual elements that would play well at scale and for a peripatetic audience. The Carr fonds contained a large selection of rich photographs, documents with interesting graphical elements and patinas that evoked their age, and reels of silent films shot on $16 \mathrm{~mm}$ that would capture the spirit of his time and the attention of the audience.

The second story we wanted to tell was that of the Archives itself by creating a porthole into the operations of the building through a circular architectural projection and highlighting the importance of the labour involved in the process of bringing attention to these archives. In this way, we wanted to capture the performance of archival work and present it with another kind of performance: the theatricality of a public projection. The Image Mill provided some inspiration for this in the way the archival images collected and processed in its presentation were animated and made to perform across the $300 \mathrm{~m}$ wide screen, directly referencing the space around it. In presenting the work of the archives in this way, we saw ourselves as the last link in a chain of labour and inspiration that began 
with Carr's documentation, continued through its generous donation by his family, careful processing and preservation and digitization by Archives of Ontario staff, and finally its presentation centred upon a large-scale public projection adjacent to the very site of the Archives. The mobile website we created for the project, containing scanned versions of various documents, letters, and newspaper clippings from Carr's journey, served as a vehicle to make these images available to spectators for closer inspection or to be circulated: a "post-production" (Steyerl quoted in Shental 2013) opportunity that opened the archives to wider scrutiny and circulation. The mobile site provided yet another window into the archive and engaged a composite dispostif (Verhoeff 2012) at the site that would re-centre audiences to this material as they moved through the web and the campus.

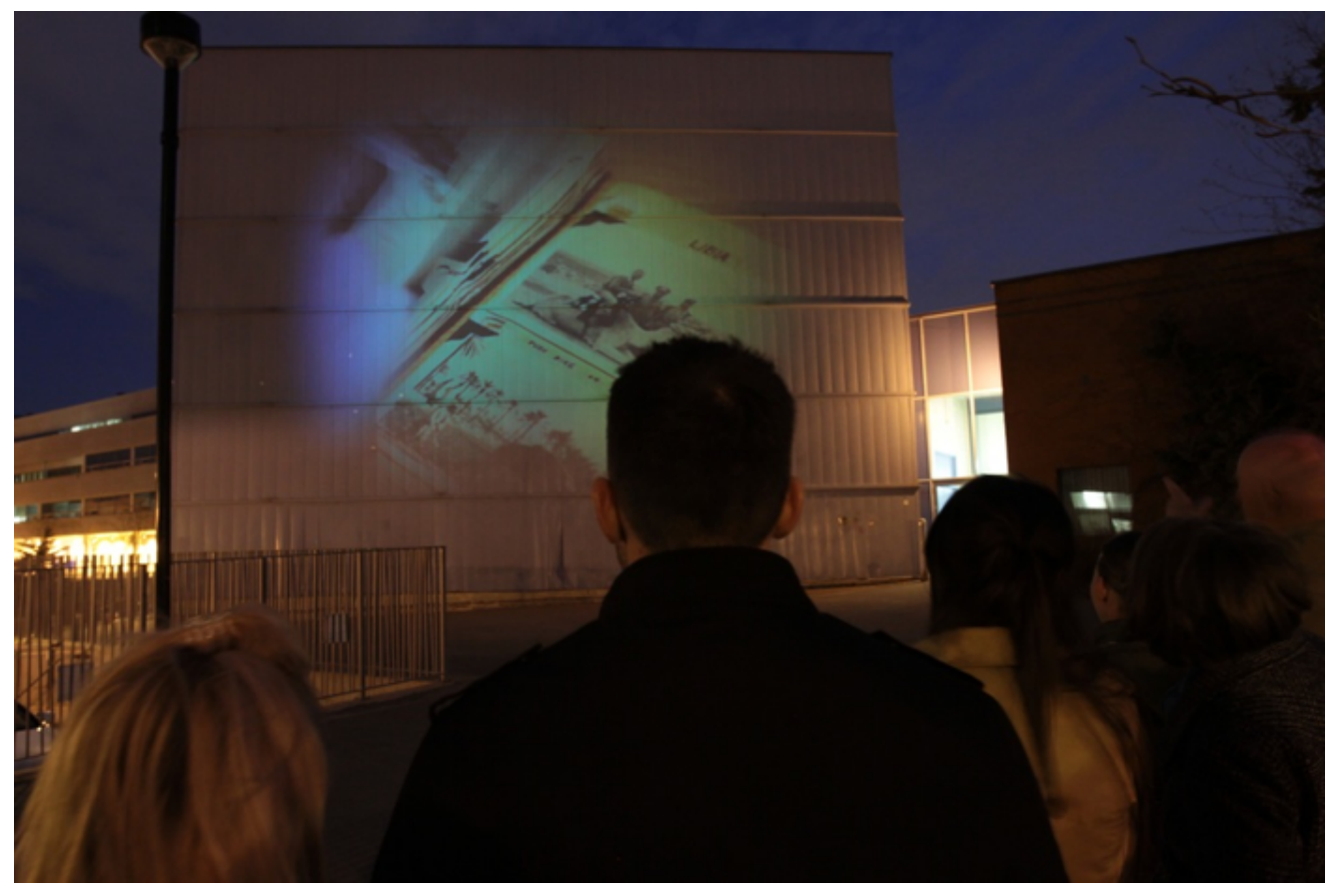

Figure 16. Dave Colangelo and Patricio Davila, 30 moons many hands (2013), installation view. Photo: Jamie Webster. 
30 moons was presented at the HASTAC 2013 digital humanities conference, from April 25-28 at York University in Toronto. It attempted to "ventilate" the Archives, that is, it attempted to create an ontological bridge that connected the interior of the Archives to the public space around it through superimposition in order to narrow the perceptual and ontological gap between Carr's story, the Archives, and the campus-between mediation and the built environment. Stylistically, we used the metaphor of the "porthole," shaping our projection to appear within a circular vignette to emphasize this encounter between the ontologically disparate spaces and times. This superimposition was extended into real and virtual space by the use of voice-over narration and a soundtrack that filled the courtyard in which it was presented, an important element of the dispositif that drew spectators to the image and helped to maintain their attention. Since views of the projection were limited to the courtyard, we did not have to consider adding a mobile component for the audio as they had in The Image Mill. Instead, we worked at a scale closer to that of McLarena which focused on attracting and maintaining viewer attention in a small space through nonindividuated, coordinated image and sound. One fortuitous superimposition that contributed to the spectacle of our presentation was the appearance of a full moon in the night sky for the three nights of our installation. While we did not plan on this occurrence, it pointed to the syncretic possibilities of spatial montage that are opened up by massive media, much like the juxtaposition of projected and real ships (and their sounds) in the projection of The Image Mill. 


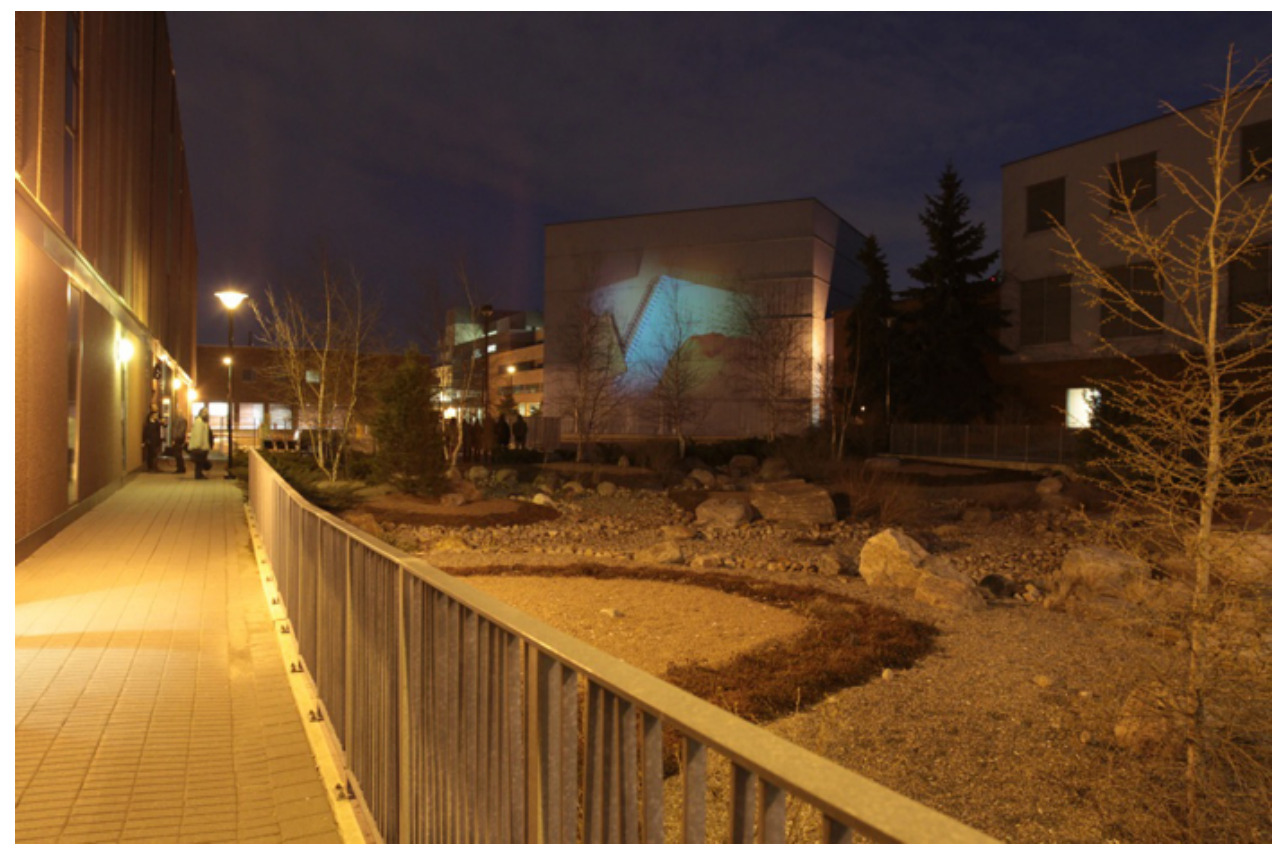

Figure 17. Dave Colangelo and Patricio Davila, 30 moons many hands (2013), installation view. Photo: Jamie Webster.

In terms of monumentality, 30 moons was designed to appear at a specific scale and in a particular setting in order to engage the displacement and condensation that Lefebvre (1997) notes of monumentality in general. Scale and setting condensed the space between the mass of the Archives building nearby and its projected extension, displacing the borders between part and whole. It allowed for the transfer of power of one institution to the other and vice versa: the authority of memory was transposed onto public space and the power of the public sphere was brought to witness and engage with the Archives and the stories contained within. This connection was also reinforced through various rituals, an important aspect of monumentality. These rituals included the rituals of cinematic spectatorship, of congregation in public space, and of interacting with mobile media. Together these reinforced the composite nature of this massive media installation, as well 
as the "accelerated" (Crary 1999) rituals involved in it. While these rituals might be seen as distracting and dehistoricizing, they can also be seen to better reflect and harness the affordances of hybrid, relational space, thus rehistoricizing a space by focusing not on the loss of sites of memory by way of the media trace (Nora 1989), but instead by maximizing the potential of the transmissive and syncretic qualities of all of the elements involved: public space, projection, networks, mobile ubiquitous media, and sound. 30 moons hypothesized that public projection, coupled with a mobile-enhanced website, could uniquely contribute to collective memory and communal experience to better reflect contemporary culture lived in and through the moving image and networked devices. It used the collectivity and publicness of the campus and the Internet as a springboard for publicizing not only the story of Douglas Carr but that of the Archives as well.

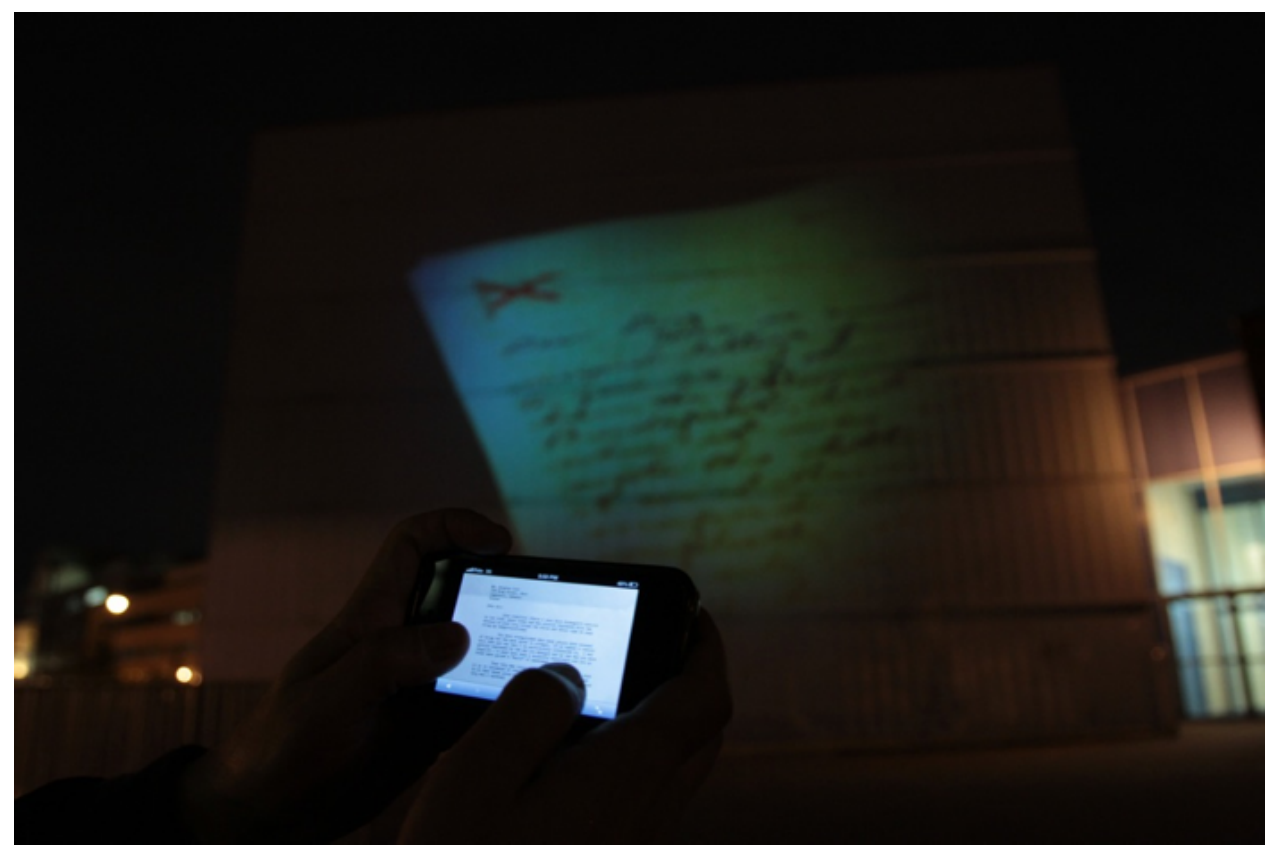

Figure 18. Dave Colangelo and Patricio Davila, 30 moons many hands (2013), installation view. Photo: Jamie Webster. 
Directly witnessing the attention that we captured by presenting moving images and sound in the space and at the scale that we did taught us a number of lessons. While we presented this work on a university campus after the academic year at a time when few students were around, we were still able to draw people into the work by the public address created with sound and image. One of the weaknesses of the project was that attention waned quickly and many people did not stay to watch the entire 15-minute loop. Our project lacked a physical referent at the site-a didactic explaining what the project was about. Instead, we had to rely on people searching online for the title of our project (prompted by the title sequence of the projection or by printed posters in the vicinity) and finding our website. This proved to be an unrealistic expectation. To solve this, it is important to place more information at the site or to have a project run for a longer period of time to allow for information to disseminate through word of mouth or through stories of people that had already experienced it, as was the case in both The Image Mill and McLarena. The Image Mill and McLarena also benefitted from promotion by tourist boards and dissemination through print, broadcast, and online media. Repetition, at the site and beyond, is a key element in entrenching in a space and in an audience the messages, meanings, and practices intended by the creators of otherwise ephemeral massive media.

Another weakness of our project was the lack of comfortable seating in the courtyard that could have helped to recenter our audience. With more funding (the project received a small amount of funding from York University as well as the in-kind donation of the space and the projectors), we would have installed bleacher seating in front of the projection to indicate to people that they were welcome to stay and watch the presentation. 
This was a tactic employed skilfully in McLarena in the tiered seating, which was placed strategically so that people could watch both the projection and the performances of participants in the mobile video booth.

Designers of The Image Mill had to consider the scale of the entire city, working with existing architectonic and geographic elements, and they had to design the space around the screen as such strategically. This led to their taking control of the street lighting across from the projection, providing ample signage, engaging tactile cues such as the subwoofers that vibrated in the viewing area, as well as placing the projection at the foot of a hill that naturally provided a kind of tiered seating for thousands. The importance of the elements of apparatus, borrowed from a history of spectatorship and specifically from a history of cinema, cannot be underestimated in creating a successful public projection: the space around the screen must be considered as carefully as the screen itself.

One final regret, limited again by time and money, was that we were not able to develop a means in which to incorporate more participatory elements into the presentation. This certainly would have helped to recenter the audience and would have better reflected the digitality in which they, and these images, now exist. Our concept of presenting "many hands" was meant to include the hands of the participants themselves, evoking a sense that their hands were part of a continuum from the hands of Douglas Carr, through the hands of the archivists, and finally our hands as researchers and artists, that could be connected through digital means and seen, altogether, as a public good that could connect us more strongly to the past. Our mobile-enhanced website represents a small concession towards this, but a deeper involvement with the artifacts, perhaps a means 
to call up 3D-scanned objects on the screen and observe them from different angles, or an interface in the form of a globe that spectators could rotate to engage specific locations on Carr's journey, would have helped to strengthen the connection between the content and the audience.

\section{The Line}

With our next project, Davila and $\mathrm{I}^{4}$ attempted to question the spatio-political assemblages of the suburbs in a site-specific exhibition using a mixture of sculpture and outdoor projection. The Line $(2013)^{5}$ was a two-part installation that included a 70 -foot long snow fence set in a marsh and a 15-min outdoor projection on a 200-year-old barn, both on the site of the Markham Museum, a historical recreation of a pioneer village in the heart of one of North America's fastest growing suburbs. It was presented as part of Land|Slide: Possible Futures, an exhibition that explored issues of land-use, food security, and cultural history. We chose to base our installation on the concept and image of the snow fence. Snow fences are built to shape the way drifting particles settle, usually to make roads safer for fast moving cars travelling in a straight line. It seemed fitting to include this as an iconic figure for our critique of land-use and migration, the loss of arable land, and suburbanization. The projection told the story of the snow fence travelling around Markham, stopping at strip malls, parking lots, housing developments, farms, hydro fields,

\footnotetext{
${ }^{4}$ Patricio Davila and I collaborated on The Line (2013). We were equally involved in early conceptual meetings with Marchessault and other artists and curators. We were also equally involved in the concept, design, and planning of the installation as well as in the construction of installation materials and the production of the sound and video elements of the piece. Davila's time was mostly spent creating and editing the final video while my time was directed towards logistical and production concerns at the site. We received inkind assistance from photographer Will Pemulis (photographic documentation) and an in-kind donation of the use of two 10k projectors from York University's Sensorium: Centre for Digital Arts \& Technology.

${ }^{5}$ See http://davecolangelo.com/project/the-line/ for documentation of the work.
} 
and so on, tracing the lines of land-use signatures etched over time. The Line attempted to ask how and where we draw the line, as the lines that manage the flow of people and things-borders, roads, bike lanes, fences, pipelines, green belts, flight paths, etc.-represent the physical manifestations of our fears and desires, and serve to shape our values and beliefs.

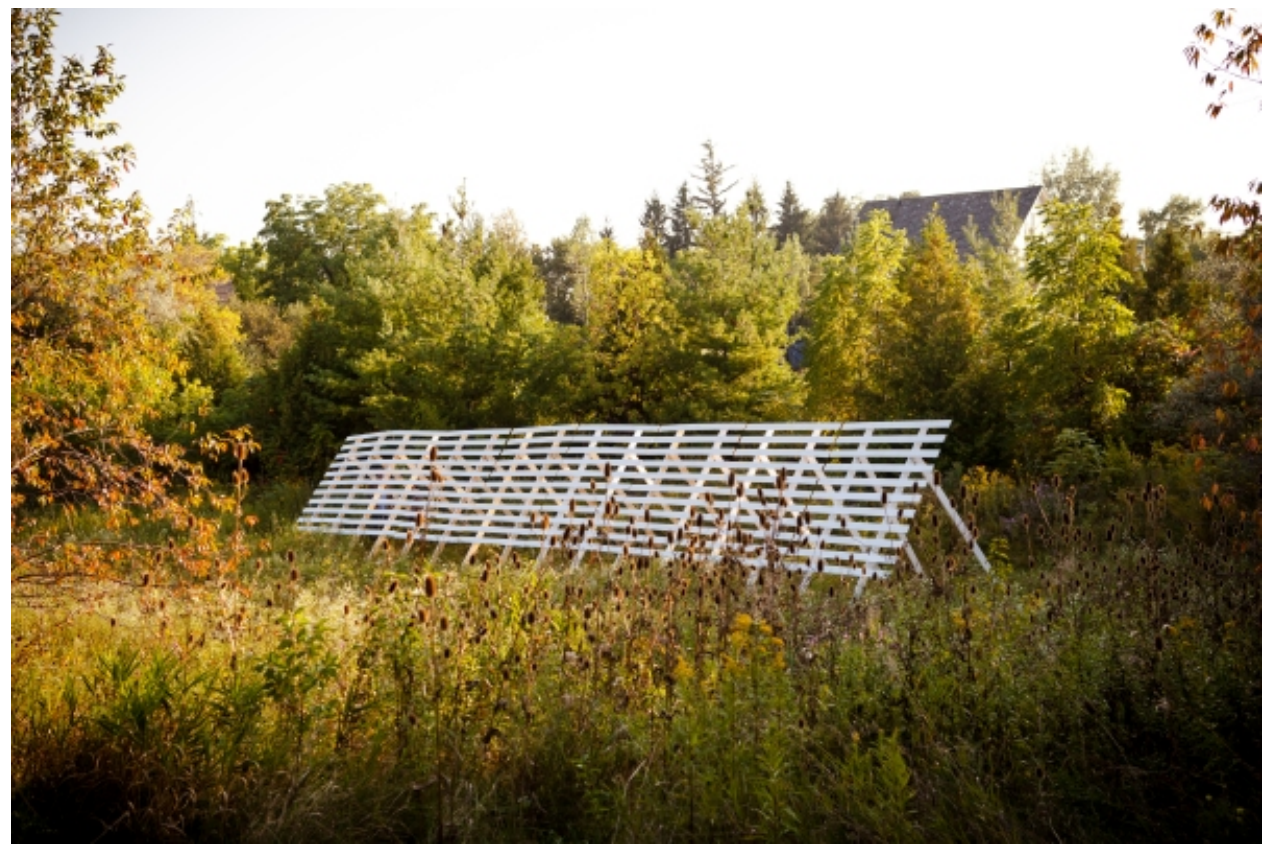

Figure 19. Dave Colangelo and Patricio Davila, The Line (2013), installation view (snow fence). Photo: Will Pemulis. 


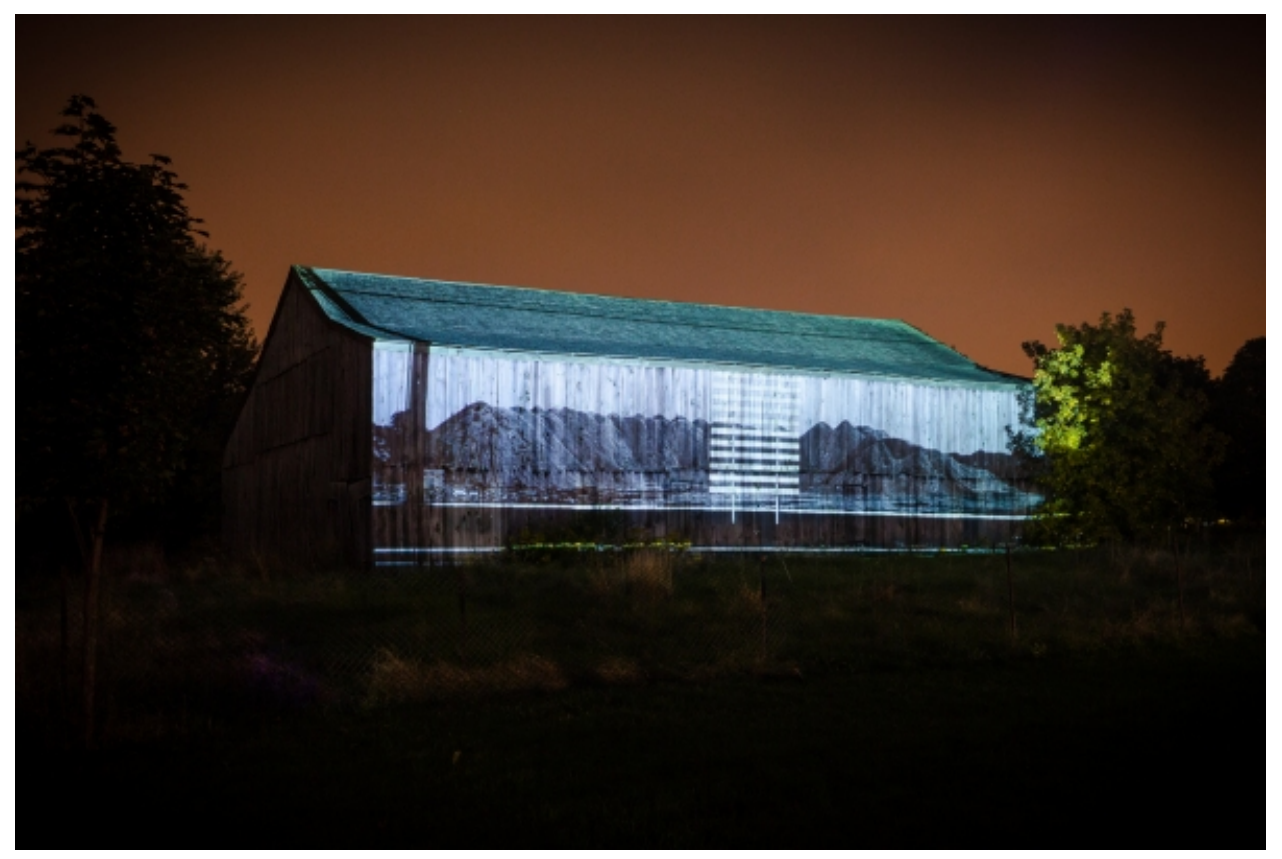

Figure 20. Dave Colangelo and Patricio Davila, The Line (2013), installation view (projection). Photo: Will Pemulis.

The Line emerged through a curatorial process led by Janine Marchessault. She approached us in 2011 and asked if we would be interested in creating a large-scale outdoor projection in response to themes of land-use, food security, suburbanization, and memorialisation at the site of the Markham Museum, a heritage village consisting of a collection of structures and artefacts from the 1800s, salvaged and maintained on the site. We were assigned the Kinnee Barn, a 200-year-old structure that had been relocated from its original home approximately $40 \mathrm{~km}$ away due to on-going development of farmland around the growing city and suburbs of Toronto.

Given the context of the exhibition and the site we were given, we decided it would be best to mirror the journey and displacement of this barn by building a large, slatted, wooden structure-a snow fence-and filming a section of it as we transported it around the 
vernacular architectural sites of Markham that included sports fields, construction sites, housing developments, and strip malls. We used the displaced barn as the canvas for our projection of the story of the snow fence traversing the lines that bisect the last available fertile land within $100 \mathrm{~km}$ of the city, transforming the landscape and its culture in the process.

A great deal of work went into recreating elements of the cinema on the barn and in the field that surrounded it. In effect, we attempted to create an apparatus to capture the attention of the audience that would be wandering around the pavilion in the dark. First and foremost, the massive projection (approximately $60 \mathrm{ft}$ wide and $25 \mathrm{ft}$ high) within the darkened space of the Markham Museum grounds provided the strongest beacon for attention, calling out to visitors from a distance in its setting at the back of the property. While this served to draw people in from afar, once spectators were within $100 \mathrm{ft}$ of the projection they began to hear the audio associated with the projection, a mix of recorded sounds from the sites we visited with the fence and ambient music, and were drawn into the ideal space for viewing the video on a set of benches-another line-that mimicked the thematic material and were embedded with speakers. Finally, the projector was carefully hidden in the attic of a nearby farmhouse, once again mimicking the apparatus of cinema in order to mystify the audience. These affordances delivered the audience to the content of the installation without undue distraction. Through these elements, we carefully extended the affective and effective use of cinematic techniques in a rural setting in order to create a composite dispositif of capture and ideological delivery. 


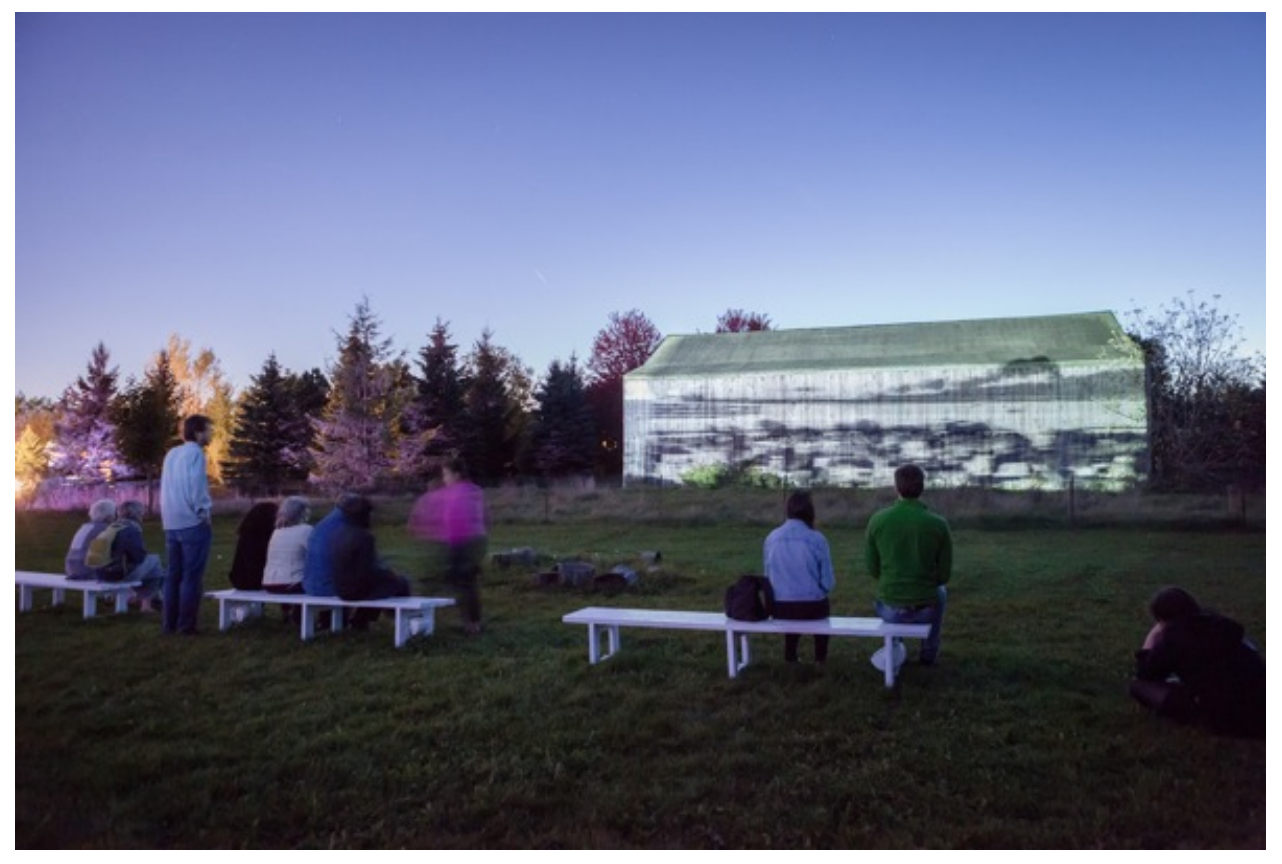

Figure 21. Dave Colangelo and Patricio Davila, The Line (2013), installation view (projection). Photo: Will Pemulis.

With the projection, scale was an important factor in capturing the attention of the audience. It allowed the images to exist on par with its surroundings, or perhaps exceed them in their luminescence. It also allowed us to present images of our snow fence at a 1:1 scale, thus, through superimposition and spatial montage, allowing the audience to measure their experience in reference to their bodies in the space of the outdoor museum and thus have a more embodied experience. They could understand their place within the environment of the museum and the environments we presented on screen to be connected. With a full-scale sculpture of the snow fence located in another area of the pavilion, The Line further extended its spatial montage with this physical element to make a correlation between the moving image and its surroundings through scale and correspondence, much like The Image Mill in its conscious congruencies between itself and 
vernacular architecture in Quebec City. The scale of the projection and its setting also intentionally created a communal experience and ritual, much like gathering around a campfire, in which these scenes and ideas would be witnessed collectively, allowing viewers to reflect on the collective impact of their actions on the environment and their lifestyles over time. Here, the collectivity of experience should not be downplayed in the experience of large-scale outdoor projections: the very basis of a Habermasian public sphere is one in which people are exposed to a specific discourse through media and are afforded channels through which to debate and discuss therein. While this can be achieved asynchronously as it most often is in online environments or in separate environments as it is in televisual broadcasts, a unique sanctity and gravity is afforded to the communal spaces of public address that massive media tap into. By virtue of its publicness, presentations such as The Image Mill, McLarena, and The Line attempt to engage and entrench the sense of shared history that only an amplified public address can afford.

In addition to the communal aspects of public projection engaged by The Line, the installation also attempted to present the memory of a structure to be something contained within the structure that could be made public through projection, thus enacting an ontological bridge through superimposition. Essentially, The Line, like The Image Mill, evoked what building may have witnessed in its history. At the very least, the audience witnessed the conditions that relate to the building and its situation, displaced as it was by development and located within a heritage recreation site, and could measure their own bodies and experience against this. In this way, The Line demonstrated the potential for a rehistoricization of space, both at the site of the projection itself, but also reaching beyond 
it through displacement, condensation, metaphor, and similarity (Lefebvre 1997). It demonstrated, like McLarena and The Image Mill, that media in public space need not necessarily estrange us from the terrestrial plane as Virilio (1986) once noted: it can show this terrestrial plane to be processual, relational, malleable, and personal through ambivalence and contingency. Through projection at the scale of architecture, every structure might be seen to be a monument or memorial in the making, its potential waiting to be unearthed, gathered from its surroundings and/or archival wake, and represented directly on it to allow for its reanimation and collective embodiment in shared memory.

The Line improved upon our experiments with 30 moons in that it provided a clear and comfortable space for congregation, enhanced by the localized provision of sound. This allowed us to capture a peripatetic audience in this semi-public space and to hold them within the embrace of our installation despite it being located in the middle of an open field. The Line also benefitted from the repetition and longevity afforded to it over the course of the installation, allowing visitors to visit and revisit the installation at their leisure. Spectators were also primed for the content through the exhibition program and by the didactic information at the site. Certainly, the success of a large-scale public address depends on the space around it, which includes the spaces of permission, dissemination, and curation that allow it to exist and flourish, legitimizing its place, and entrenching it within a space in memory despite its ephemerality.

What The Line was not able to achieve, again due to time and budgetary constraints, was, once again, some aspect of interactivity akin to the interactivity that contributed to the success of McLarena. We had initially toyed with the idea of having 
various segments of the snow fence in The Line appear or disappear depending on the proximity of the viewer to the screen, or to have pressure sensors embedded into the benches to cause different video segments to appear as viewers sat down to view the work. This would have integrated the viewer more directly into an identification with the images and their role in engaging the various lines that criss-cross the landscape, expressing and constituting the conditions of suburban life. It would have also created the possibility for cooperative play and greater communal identification with the images if the simultaneous, coordinated efforts of spectators (all approaching the screen at once, or all sitting down across the benches at the same time) could have been reflected in coherent line segments or could have triggered hidden material. This would have better approximated a democratic surround and an activated spectatorship. The message of such coordinated efforts would have helped to instil a sense of empowerment and responsibility in the construction of (semi-)public space (through the construction of the public image), thus approximating a political position through massive media, a democratic surround in which our collective future and past might be better expressed through the public, interactive image.

\section{Conclusion: A Perceptual Laboratory for Popular Needs and Aspirations}

The new assemblages of media, architecture, and space centred upon the composite dispositif of large-scale public projection-one form of massive media-advance the practices of monumentality and the development of public culture. In their efforts and ability to interface with space, something we can analyze through lenses borrowed from film studies, 
namely superimposition, montage, and apparatus/dispositif and through direct engagement with the medium via creation-as-research, these installations create ontological bridges between diegetic and the extra-diegetic realms, suturing them together such that a hybrid and relational space is created between the moving image and its surroundings, merging the logic of the screen and the logic of architecture and monumentality. The affective and attractive potential of the screen, particularly when it is coupled with the manipulation and networking capabilities of digitality, are merged with the solidity, visibility, history, and stature of public spaces, their buildings, and their objects and sites of public representation. The new narrative and associated potentials that emerge from this confluence lay the groundwork for the possibilities of rehistoricizing a space, that is, of opening it up to alternative meanings, relational identity formation, and unexpected encounters with historical and archival site-specific material. In many ways, massive media, still in its nascent phase, mirrors the early days of cinema in which the medium both oriented and disoriented audiences, providing a "perceptual laboratory" (McQuire, 2008, 114) in which audiences could understand time, space, identity, and the city, in new ways. The examples presented in this chapter begin to outline some of the possibilities for this new perceptual laboratory. For example, The Image Mill attempted to capture a peripatetic audience by engaging a composite dispositif that incorporates various combinations of sight, sound, scale, and touch, in order to approximate a democratic surround, that is, to provide multiple sensory inputs that the viewer recombines in their own unique way. Works such as McLarena at the Quartiers des spectacles extend the democratic possibilities of the medium by incorporating a more active spectatorship 
through direct participation in and through the image. Critical and creative practices with massive media run counter to the equally plausible and probable expansion of advertisements that tap into the novelty of the form, as well as its affective potential generated through the moving image, to recenter the generalized audiences in urban spaces towards a capitalist address. That said, due to the high cost of producing and maintaining large-scale public projection works, projects such as The Image Mill and McLarena invariably rely on public funds and access to crucial zones of commerce and tourism in cities, and thus must also remain, to a degree, vehicles for the promotion of the city as an attractive, engaging, progressive destination: a perceptual and commercial laboratory.

Regardless of their purpose and content, all massive media works of large-scale public projection challenge the naturalized exclusions of public space, that is, they begin to allow for a certain level of ambivalence and contingency in the expressivity of architectural surfaces. They also represent a dense transfer point and focus for personal and public postproduction practices through image appropriation and remediation that serve to deeply embed architecture and public space in contemporary image practices. Beyond this, though, massive media must continue to be developed by cities, curators, and artists, to open these sites to greater experimentation and criticality and to ensure that they reflect a productive and democratic form of monumentality. Quartiers des spectacles remains an ideal model in this sense, providing year-round access and interactive programming that allows for a greater degree of artistic expression, a wider variety of interpretations of a space, both through the image and through the movement of peripatetic audiences moving through it, and a deeper development of the capacity for audiences to engage with this new 
medium. This, I would argue, is an example of how massive media can better reflect the “popular needs and aspirations” (Mumford 2000, 151) of our time. Artists and curators must see the value in developing a capacity in both public space and the public audience, on- and offline, near and far, to address the potential of an architecture and monumentality of expanded cinema and new media art. 


\section{CHAPTER 3: The Empire State Building and the Roles of Low-Resolution Media}

Façades in a Data Society

\section{Introduction}

It certainly was not the first time New Yorkers saw flashing lights atop the Empire State Building; but, it was the first time the lights danced as they did, synchronized to Alicia Keys' singing two of her songs, "Girl on Fire" and "Empire State of Mind," appropriately selected for the launch of the building's newly installed programmable LED lighting system. Amidst the ambient glow of the surrounding buildings, bright orange and red hues shifted to blue, purple, and yellow with the pulsing beats of the music. The colours mixed and faded into one another, rippling across the façade and rising up and down the antennae to Keys' voice. As Megan Garber (2012) of The Atlantic described it, it was like "a fireworks show, with the illumination in question coming not from controlled explosions, but from controlled LEDs": a firework-like show that in its stoic silence could be completely ignored by thousands while remaining a formidable centre of attention for those who knew what to look and listen for by tuning in to the synchronized audio on a local radio station, a show that rippled out further into the night and onto the screens of those near and far via YouTube (see Figure 22), Instagram, and Twitter, and Facebook. 


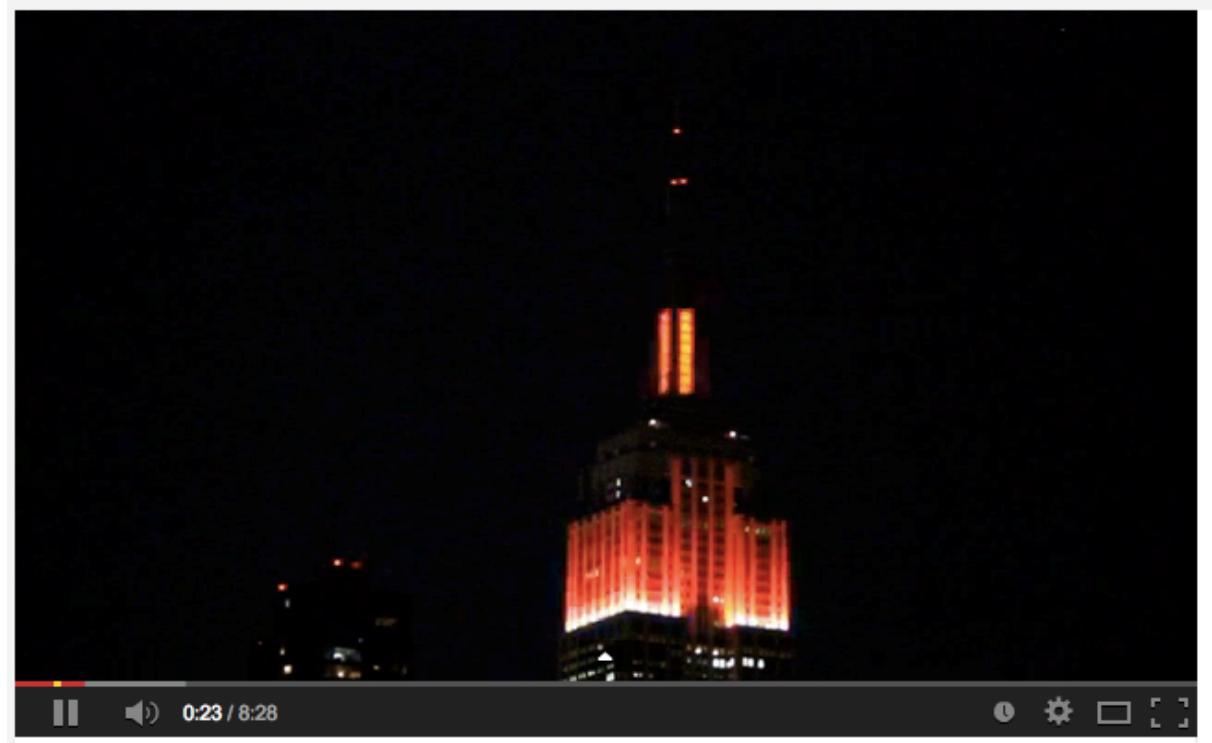

The NEW Empire State Building Lights - Live Show with Ali...

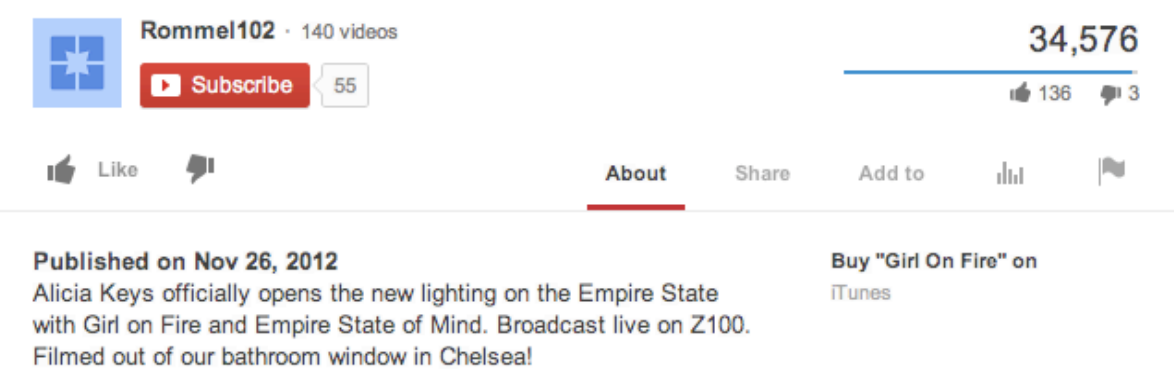

Figure 22. Empire State Building, live light show with Alicia Keys, November 26, 2012, YouTube.

That evening in November of 2012 marked the beginning of an increasingly varied and experimental program of lighting atop the Empire State Building, a distinct shift in the lighting tradition of the building that began in its first years of operation in the early 1930's. The Empire State Building's new lighting program can be seen as part of a larger shift in monumentality, architecture, public space, and digital culture characterized by the transformation of iconic buildings and significant architectural sites such as Toronto's CN Tower or Paris' Eiffel Tower by ever more elaborate and responsive media façades. An emerging collection of new, purpose-built structures, such as the Ars Electronic centre in 
Linz, Austria, and the Ryerson Image Arts Building in Toronto, have also incorporated low-resolution, expressive surfaces into their façades. These structures seek to create a connection between people and the city in order to produce new collective, embodied, and emotional experiences, variously interpolating art audiences, consumers, and a subtle mixture of the two in the process.

Not unlike their public projection counterparts, these low-resolution displays, when combined with the expanding field of digital media-the "possibility machines" (Galloway $2012,55)$ that include all manner of ubiquitous computing and sensing devices, and the social networks that encourage their situated use-contribute to the formation of a “composite dispostif" (Verhoeff 2012, 105) where screen spaces cooperate and compete in an increasingly geographically distributed, hybrid (De Sousa e Silva 2006), and relational space characterized by ambivalence and contingency (McQuire 2008). Like public projections, they incorporate and extend standardized environments of spectatorship (the city, the cinema, and the radio, for example), with their affordances and attendant ideologies in order to co-constitute a relational experience through spatial montage and superimposition. That said, low-resolution media façades can be seen to more easily incorporate social and mobile media into their composite forms, often requiring these channels to encourage engagement with their content through various levels of participation, or help decode and disseminate their ephemeral, abstracted messages to an audience that is, like public projection, geographically dispersed and peripatetic (Bennett 2009). 
Furthermore, these low-resolution surfaces, in their limited expressive capabilities, often display fluctuations in one or two variables at most, thus creating what Davila and I (2011) call “public data visualizations," or what Vande Moere and Hill (2012) call "urban visualizations" (26), that dramatize architecture and data in order to make data more public and visible, to make the city more expressive, and to involve citizens in urban issues in novel ways. Public data visualizations represent a unique confluence of data, networks, architecture, and public space, allowing for the mapping of context and site-specific data in real-space and real-time onto physical space in ways that can be engaged on- and offline. Thus, public data visualizations, like public projection, can also engage temporary publics and reimagine monumentality by creating focal points for transversal communication that combine elements of democratized urbanism, collective memory, debate, emotion, control, and commerce.

Finally, as they are directly integrated into architectural surfaces-as opposed to the ephemeral application of projected light-low-resolution media façades, also known as media architecture (Media Architecture Institute 2015), represent an important shift in architecture that merges the logic and presence of the screen with the logic of the building and the monument in durable, permanent ways. As such, media architecture, more consistently than public projection, evokes debates of attention and/or distraction through the increased contingency and ambivalence of architectural surfaces and the subsequent strengthening or weakening of a historical consciousness associated with space and architecture. As a result, I argue that embedded, low-resolution forms of massive media point to the development of a supermodernism (Ibelings 2002) in architecture 
characterized by the irruption and imbrication of the "infoscape" and the cityscape, where data-rich public spaces of identity, congregation, and contestation seek and find an appropriate and consistent outlet in existing or newly-constructed highly visible spatial forms.

Specifically, in this chapter, I argue that these assemblages of media, architecture, and space challenge our ideas of what public culture is, and how things like collective unity, civic pride, nationalism, and progress are expressed through space and artistic expression. Focusing on the Empire State Building as a case study, a structure with a long history of expressive, low-resolution public lighting, I trace the history of expressive lighting on the building, demonstrating they ways it has become more varied in its lighting program and more integral to the life of the city as a result. I analyze current social media traces incorporated into its lighting schemes, isolating representative examples from the official @EmpireStateBuilding Twitter and @empirestatebldg Instagram feeds, extending an analysis of the ways massive, networked, low-resolution public data visualizations now change and challenge notions of space, monumentality, and the public sphere by engaging diverse, transversal, and sometimes participatory publics. More practically, I explain, by way of a number of examples, how data, networks, and animations combine to create massive media that are situated, functional, and informative (Vande Moere and Hill, 2012) and how they are shaped by and relate to their environment, content, and carrier (Vande Moere and Wouters, 2012). Finally, by presenting two creation-as-research works, ETOWER (2010) and In The Air, Tonight (2014-), I argue that the preceding characteristics can be applied to evaluate the efficacy of low-resolution media façade installations while 
also arguing for the importance of experimentation in this new medium, one limited in its expressive register and primarily attached to highly controlled and corporatized spaces that often preclude alternative uses. This can be achieved through persistence, an activist attitude, and well-informed and funded curatorial support (discussed in greater detail in Chapter 4).

\section{A Short History of The Empire State Building}

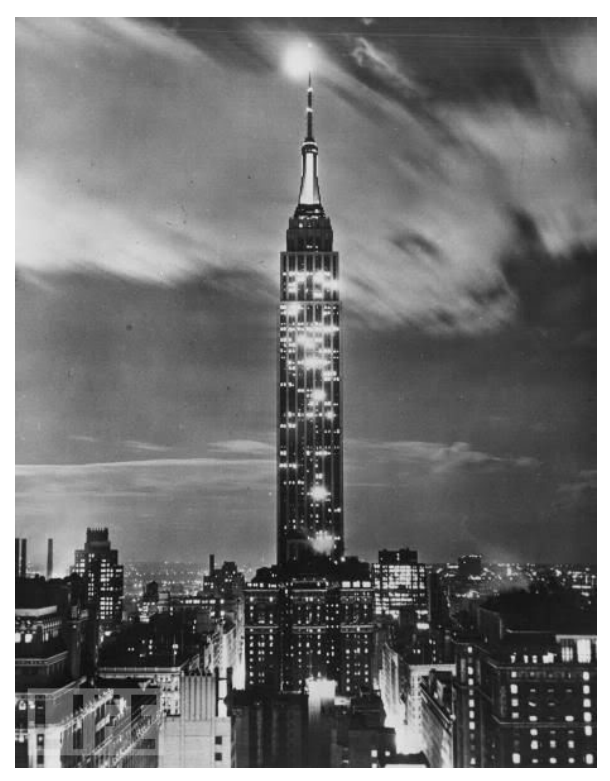

Figure 23. The Empire State Building, January 1, 1932.

Although completed in 1931, it was not November 1932 that the Empire State Building (see Figure 23) installed its first proper lighting system. A searchlight beacon was mounted at the top of the building to mark the election of native New Yorker, Franklin D. Roosevelt, to the office of the President of the United States of America (Empire State Building 2014). In this new role as iconic information signal the building showed Americans that they were united under the guiding light of a new administration, 
performing the displacement and condensation that Lefebvre (1997) describes of monuments: condensing an entire nation's desires onto the spire of the building now imbued and expressive of the spirit of a nation. Furthermore, by combining the celebration of an election with a powerful symbol of civic pride, the lighting designers and management of the Empire State Building intensified the meaning of both in the process (Rowlands and Tilley 2006), transferring power from the office of the president to the building and back again.

The combination of architectural lighting and information was far from a novel phenomenon in New York in 1932. Other building types and lighting configurations dotted the skyline with dynamic displays. Architectural lighting was a mark of sophistication and status for a building and the city. The dynamic floodlighting of the Empire State Building simply allowed the icon to "join ranks with others of its age" (Tauranac 1995, 188). In fact, the original plans for lighting the Empire State Building were influenced by the appearance of communicative and dynamic context-specific light displays on other prominent buildings. Since the 1920s, the Metropolitan Life tower on Madison Square and the Con Edison Building on Fourteenth Street flashed white every hour and red every quarter of an hour (Tauranac 1995, 188). Douglas Leigh, the man responsible for creating numerous advertising "spectaculars" in Times Square and Broadway in the first half of the twentieth century, sensed at the time "... an insatiable yearning for constantly knowing the time and temperature" (Sellmer 1946, 48) amongst the rapidly growing crowds in Manhattan. Consequently, a seven story high thermometer 
for an ale company and a clock for the Gruen watch company were among Leigh's many proposals for clients at the time.

The Canada Life Building in Toronto was another example of the early use of expressive architectural lighting to draw attention to buildings by providing timely and noteworthy information. Although the building opened in 1931, it wasn't until 1951 that a $12.5 \mathrm{~m}$ tall spire of lights was installed on its roof, $98 \mathrm{~m}$ above the street.
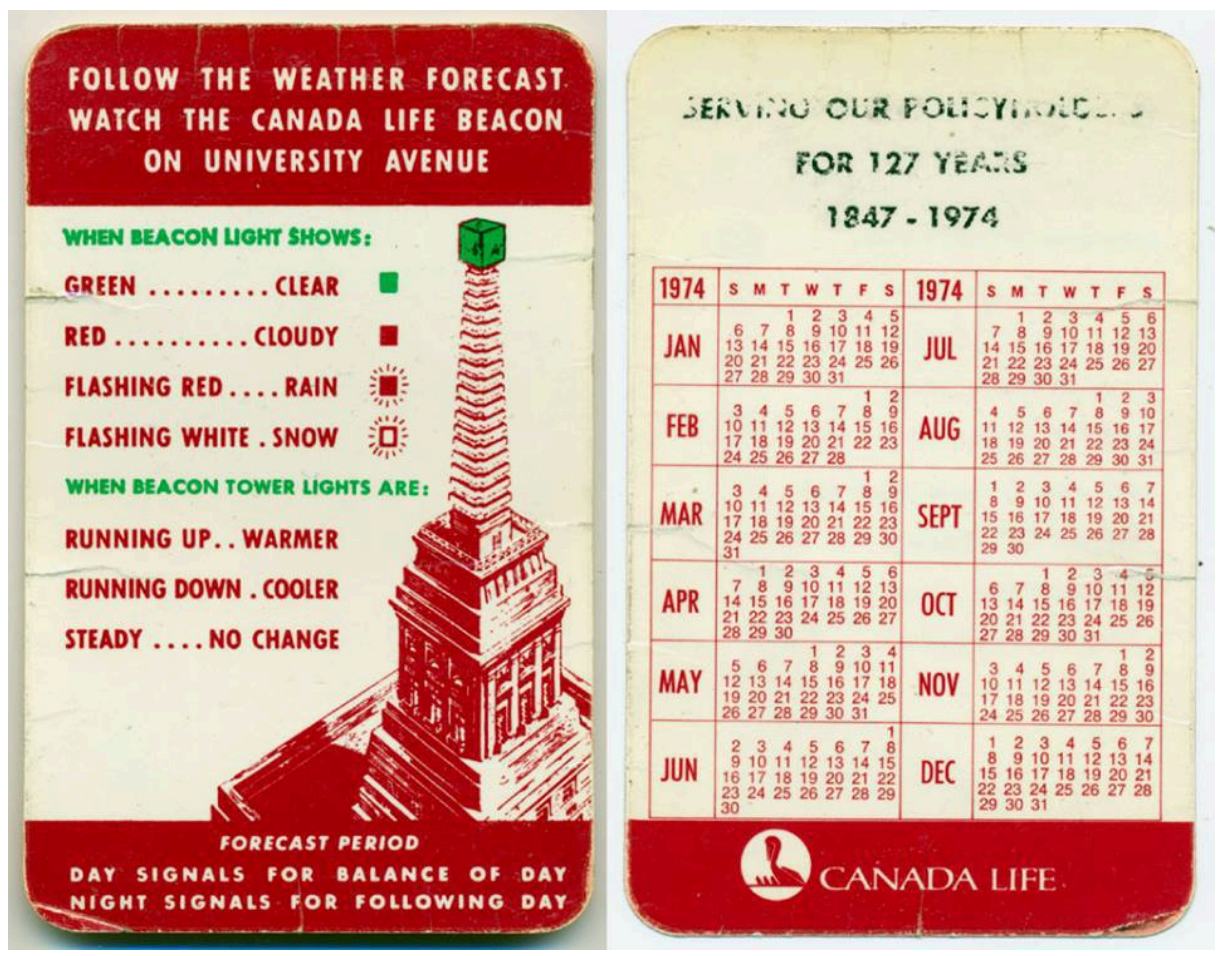

Figure 24. Calendar card for Canada Life Building with weather beacon guide.

The lighting system featured a long stem of yellow lights that were timed to create a rising or falling effect depending on the forecasted temperature. It also featured a tip that flashed red or white, indicating rain or snow respectively. Solid green meant the weather ahead was clear, and solid red meant it was going to be overcast (see Figure 24). The president of 
Canada Life, E.C. Gill, told the Toronto Star in 1951 that the building should provide both a useful service as well as a point of general interest and connection for people of the city. Gill added, "For most people the weather provides a topic of conversation at all times" (quoted in Bateman 2013). Expressive lighting enhanced the function of civic architecture, making it both a point of reference geographically but also spatially and socially by combining site and context-specific information with the status and visibility of a building.

Thus, from an early stage, architectural lighting could be seen as unifying, informing, and stimulating conversation amongst city dwellers while also elevating the esteem of structures and the organizations attached to them. Beyond mere nodes and landmarks (Lynch 1960), these buildings became communicative beacons that captured attention through the contingency and context-specific relevance they presented, introducing elements of ambiguity, expressivity, and participation into practices of monumentality. Additionally, by presenting a topic of conversation, architectural lighting enhancements went beyond providing useful information by creating a public, that is, a group of people witnessing and discussing the same information at the same time (Warner 2005). Rituals of collective activity were stimulated by these structures and the structures became, as Warner (2005) points out of contemporary public spheres after Habermas, more emotional, playful, apolitical, and agnostic. Early public data visualizations created and re-centred publics by providing dynamic information that prompted urbanites to look with regularity and to share the implications and outcomes of this contextually relevant information with others, thus transforming the social life of the city, its inhabitants, and the status of its buildings. 


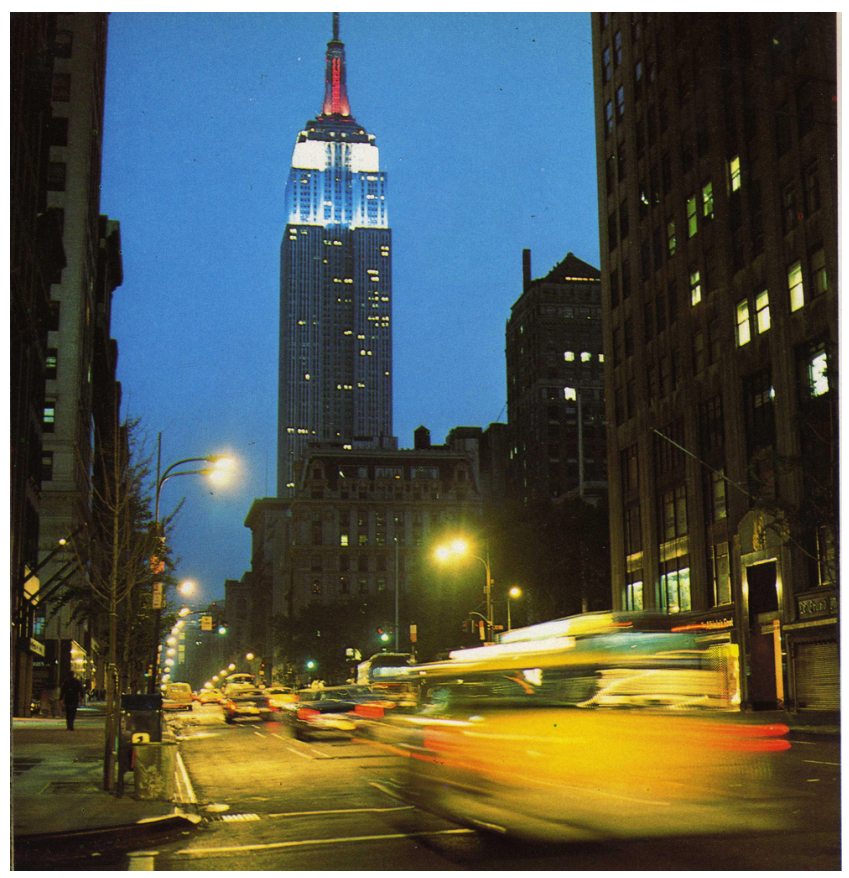

Figure 25. The Empire State Building's "Freedom Lights” in 1976.

\section{Colours and Meanings}

In 1976, Times Square's lighting impresario Douglas Leigh was serving as chairman of City Décor for the National Democratic Convention and the Bicentennial that year. Having once been denied an opportunity to work with the building's lights, ${ }^{6}$ Leigh was determined not to be turned down again. While looking for a way to celebrate the Bicentennial events he was presiding over, Leigh asked management to change the white lights atop the building to red, white, and blue (see Figure 25). Leigh called it the application of "color with meaning" (quoted in Tauranac 1995, 358). The new scheme proved to be quite popular. Architect Charles Linn said the building became "... the toast

\footnotetext{
${ }^{6}$ Leigh had considered the Empire State Building as the canvas for one of his "spectaculars" but his pitch was eventually denied due to citywide restrictions resulting from America's entry into World War Two. As Tauranac (1995) notes about this failed pitch, "His dream was to light it like a cigarette, with ashes at the top and smoke curling up into the heavens, an advertising colossus created by a million white electric bulbs, a few thousand red lights to paint a burning tip against the night sky, and the Lucky Strike name emblazoned in neon on all four sides" (313).
} 
of the town" (quoted in Tauranac 1995, 358). The success of the colour change set in motion a plan to expand the colours to include more "meanings." For Martin Luther King Jr. Day the tower glowed red, black, and green; for Valentine's Day, red and white; for Easter Week, white and yellow; for Police Memorials, blue, and so on. As Tauranac notes, “These colours became traditional as did celebrating specific events" (Tauranac 1995, 358). In addition to these traditions, new uses for the lights and their semiotic potential were explored. In this inaugural year of expanded programming, a creative solution for celebrating the Camp David peace accord was presented where two sides of the building were lit in the state colours of Egypt and the other two lit in the colours of Israel (359). In their expanded format the lights now inserted the Empire State Building more deeply into the fabric of the city and culture creating a focal point for a growing list of events, milestones, holidays, and causes. This expansion was limited only by the investment of time and money required to change the lights-a six-hour process that called for a team of at least six workers (Collins 2007). Over time, the building had become more sensitive and expressive of its unique time and place, growing into its new role as a monumental marker of contextually relevant information. 


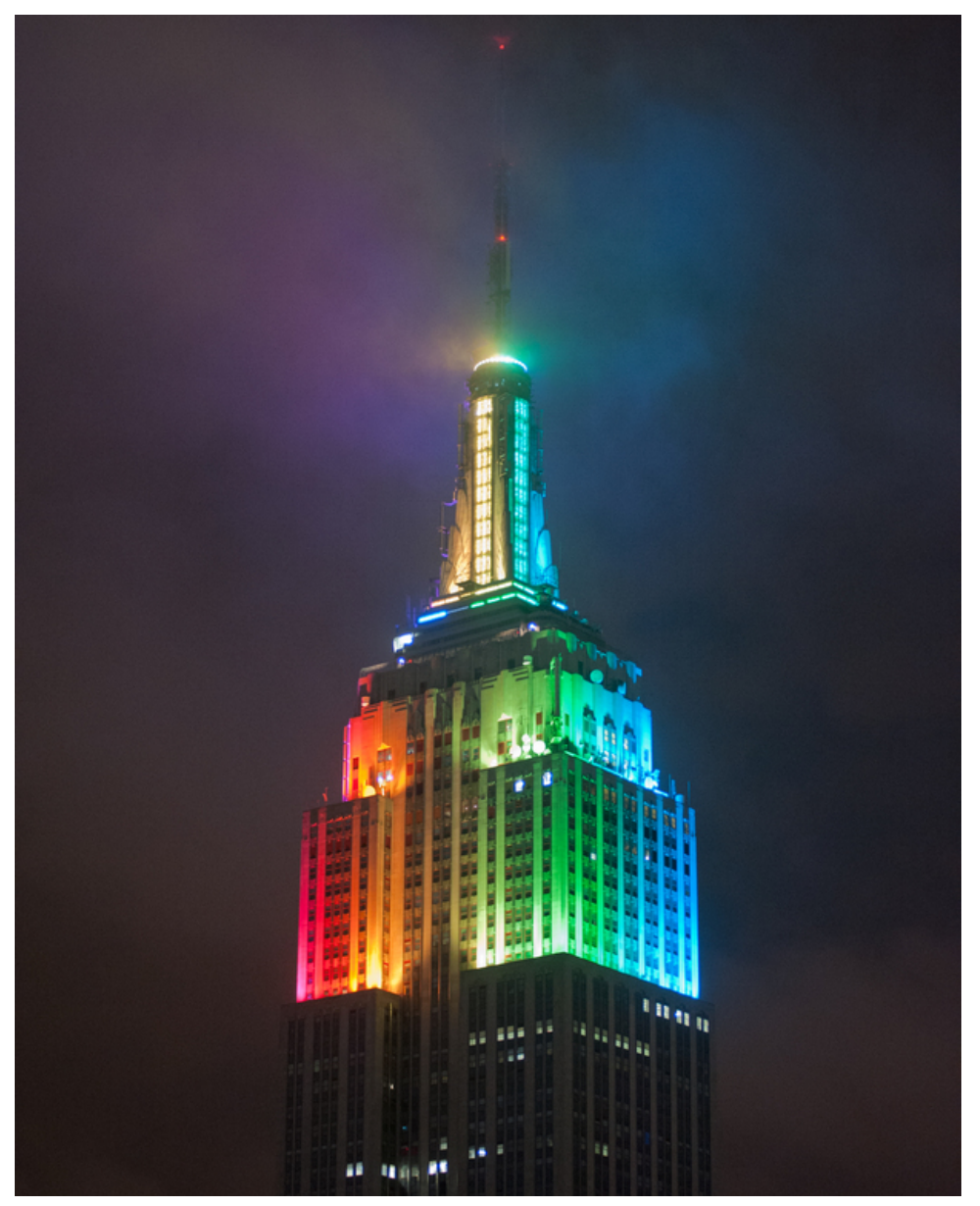

Figure 26. The Empire State Building with Philips Color Kinetics System.

Indicative of the growing popularity of the practice of expressive architectural lighting and improvements in lighting technology, in 2012 the Empire State Building installed a Philips Color Kinetics programmable LED lighting system (see Figure 26) capable of displaying an array of over 16 million colours in "virtually limitless combinations," all at the push of a "computer controlled” button (Philips 2012). Shortly after this change, the lighting system was called upon for yet another presidential election. Instead of simply and poetically indicating the final result as it did 80 years earlier, the building was programmed to display an up-to-the-minute tally of votes during election night 
coordinated with real-time coverage on television, the Internet, and social media (see

Figure 27). The CNN (2012) Press Room website described the lighting event as such:

The four-sided tower, which sits atop the building more than a quarter of a mile from the streets of Manhattan, will be illuminated in patriotic red, white and blue vertical stripes, while the mast will be lit in blue and in red on two sides each to represent President Obama and Gov. Romney's respective electoral vote totals. CNN, which will exclusively shoot footage from the rooftop of a neighboring building, will air live images of the illuminated tower as the evening's results progress. When CNN projects a winner of the presidential election, the tower lights of the Empire State Building will change color to all-blue or to all-red.

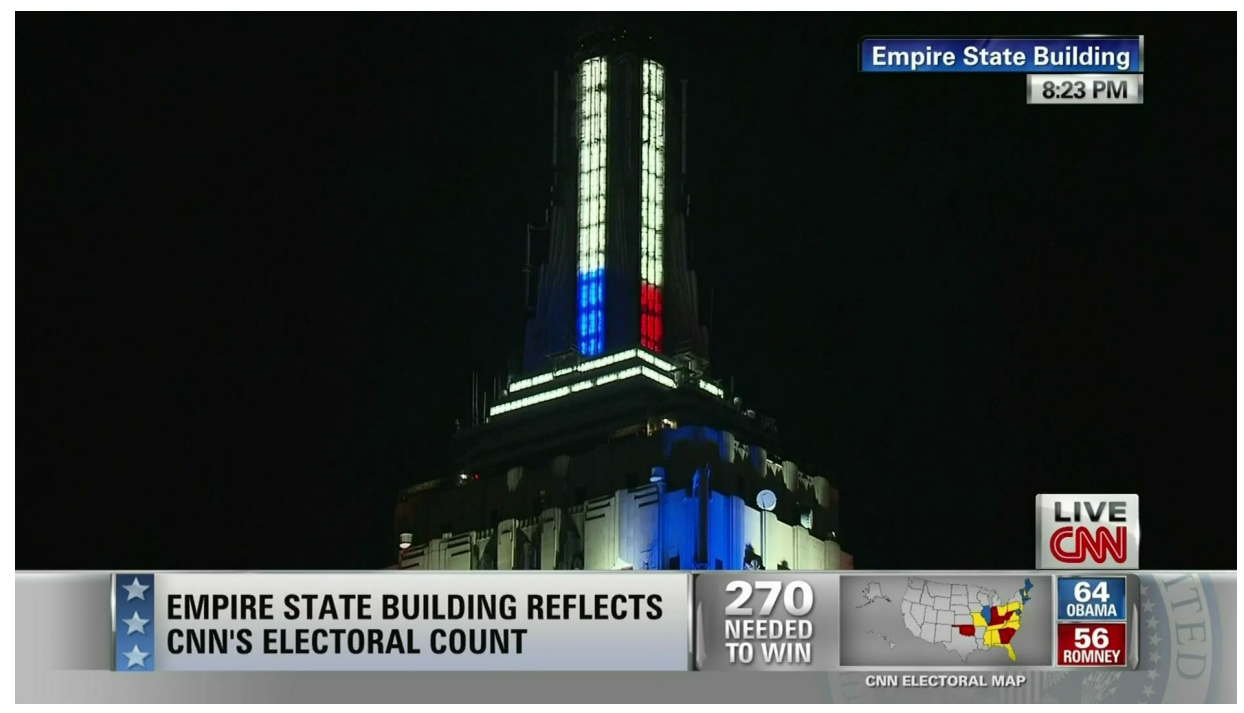

Figure 27. Empire State Building with CNN election results for Presidential election, 2012.

Changes in the media landscape over the past century (i.e., satellite television, the Internet) meant that the building would now be a central figure not only in the city, but for countless social media posts. It would also be the epicentre of attention and action for one of the world's largest news networks on one of the biggest news nights of the decade-a focal point in a global composite dispositif. It could call out to and capture an audience in a number of coordinated ways, drawing them in with the promise of real-time updates, 
animation, and participation. The level of detail of its message, its amenability to appropriation and transformation by media outlets and viewers, had changed (see Figure 28) greatly since 1932, and its status as a global icon was supported and reinforced in the coordinated and distributed mixture of localized and globalized communication.

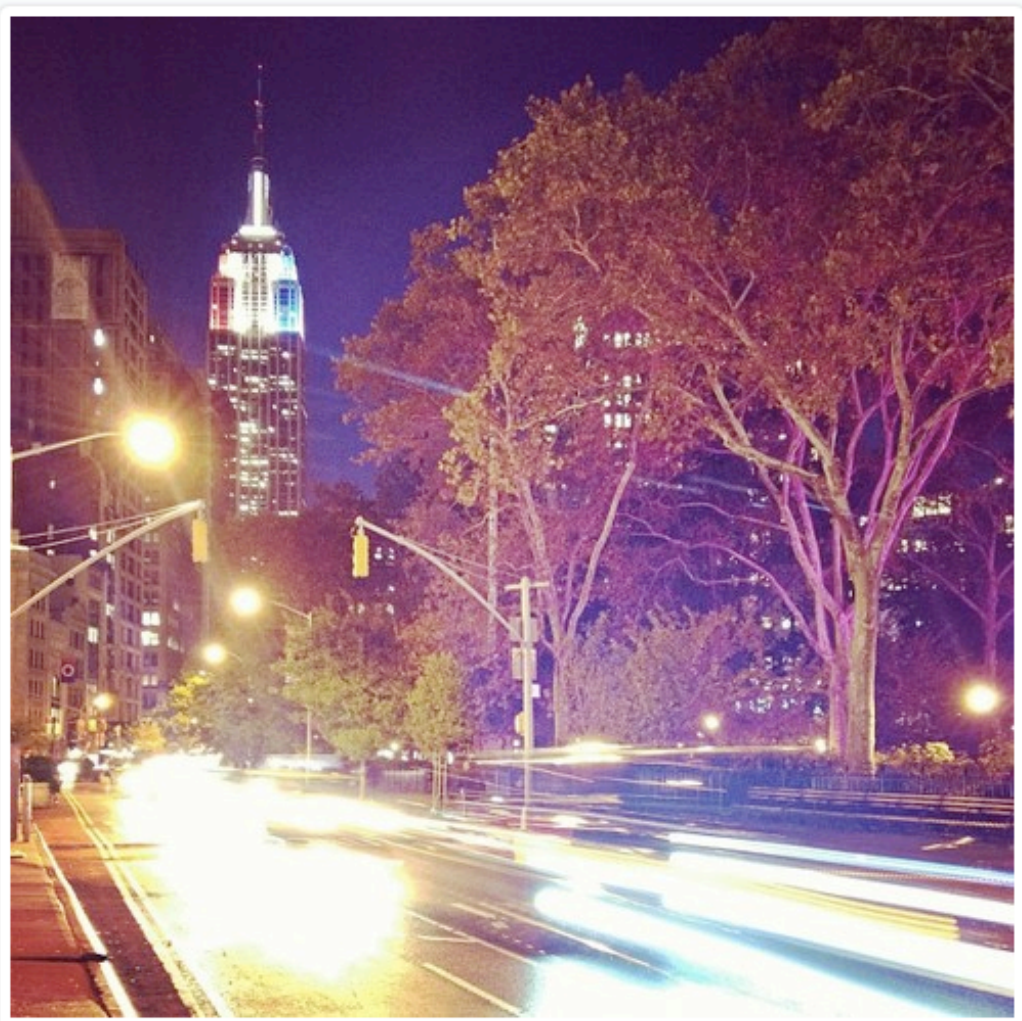

Hey New Yorkers, look up tonight! The spire of the \#EmpireStateBuilding will be lit up as a red and blue bar graph, which reflect @ CNN's electoral vote projections Photo taken this evening on an iPhone as a 1-second exposure using the \#NightCap app and a \#gorillapod tripod \#CNNelection \#Election2012 (5) SGoralnick : A Year ago

Figure 28. Screen capture from 'sgoralnick' during the 2012 election, Instagram.

The agglomeration of media saw the Empire State Building assume a prime position during the 2012 election as a massive data visualization-a big canvas for big data (and big business). The Alicia Keys event that officially kicked off the new lighting scheme 
further demonstrated how programmable low-resolution façades have expanded the role of architecture in contemporary cityscapes. These events substantiate an important trope of massive media focused on data collection, participation, and visualization in order to support new and contested public spheres and rituals of mass culture, collective memory, and communal experience. These are examples of Giedion's new, new monumentality (Mumford 2000) that seeks to better reflect and include a transverse community that congregates around the monumental both in person and online. Public data visualizations, a category supported directly by lighting systems such as those found on the Empire State Building, allow for expanded possibilities for the buildings and spaces they occupy and the audiences they address, enabling a re-coding of space and a recentering of local and geographically distributed audiences. As in the 1932 example, today's low-resolution media façades carry on a tradition that sees highly visible buildings as sites for the display of "public" information to be digested, consumed, circulated, and shared.

\section{Understanding Contemporary Dimensions of Public Data Visualizations}

The current complexity in contemporary expressive architecture can be theorized through the concept of public data visualization (Colangelo and Davila 2011)-that is, assemblages of light, data, and space that make data publicly visible, dramatizing architecture and data in the process. Vande Moere and Hill (2012) see the potential for public data visualizations, or what they call "urban visualizations," for improving the quality of life in cities by involving the local population in understanding the key drivers of urban issues (26). This includes constituting an "activated spectatorship" (Bishop 2004) 
and a new register for public discussion and debate via public displays of data. As such, the authors state that successful and effective urban visualizations must be situated, informative, and functional. An urban visualization is situated if it is embedded in a real-world environment and both borrows and contributes to it (Vande Moere and Hill 2012, 26). As the authors note, if an urban visualization is properly situated, it should not require much in the way of a detailed explanation to be understood. For example, the urban visualization found in Pollstream-Nuage Vert (2008) in Helsinki, an installation that incorporated laser light to illuminate a proportion of $\mathrm{CO}_{2}$ emissions emanating from a smoke stack based on local energy consumption levels, uses a symbol of energy consumption as its canvas in order to become more legible and impactful through self-referentiality (see Figure 29). An urban visualization is informative if it allows onlookers to create meaningful insights or provide feedback (Vande Moere and Hill 2012, 26). Pollstream-Nuage Vert certainly allows for the creation of meaningful insight, but does not directly provide a feedback mechanism, so it does not fully satisfy this criteria. Finally, an urban visualization is functional if it reaches a significantly large audience, does not impede other civic functions, blends into the urban fabric in a pleasing manner, provides trustworthy information by being fair and accurate, reveals its sources, and is persuasive in some way, calling for some sort of reflection, change or action (26). Again, Pollstream-Nuage Vert is functional in that its scale allows it to reach a large audience, its presentation does not impede the functioning of the smoke stack, its sources are revealed to be fair and accurate on its website, and it is certainly persuasive in terms of encouraging energy saving measures. Overall, it functions as 
a public data visualization in that it makes information more visible and legible, dramatizes architecture and urban space, and involves the local population in a key urban issue.

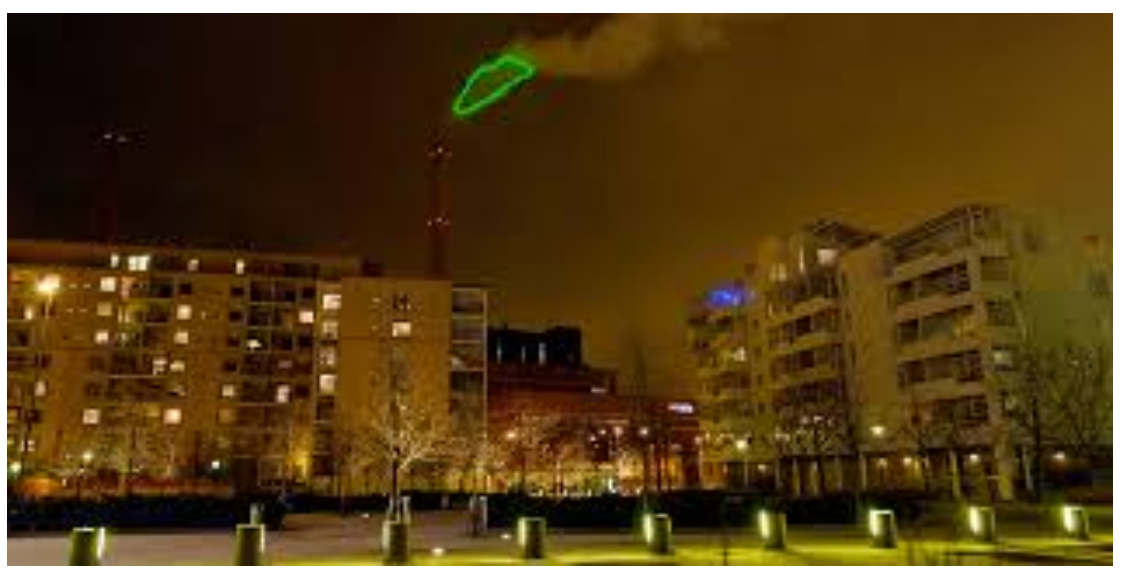

Figure 29. HeHe (Helen Evans and Heiko Hansen), Pollstream-Nuage Vert (2008), Helsinki.

In addition to functional, informational, and situational considerations, Vande Moere and Wouters (2012) discuss the importance of aligning public data visualizations with the overall context in which they exist. To unpack context, they focus on the importance of the interplay and cohesion between the environment (surrounding buildings, local culture, atmosphere), content (the information displayed and any interpretations that may be generated by it), and carrier (the building, square, façade, or any other element that supports the medium). They advocate for a "context-awareness" (1) as a design characteristic for media architecture along these axes to ease community acceptance and improve the efficacy of these structures in society so that they might be less susceptible to falling out of use or favour. One example of media architecture failing to remain sustainable due to a change in environment and carrier context is that of the Dexia Tower in Brussels, Belgium. The $145 \mathrm{~m}$ tower, equipped with individually controllable LEDs, was 
initially the site of several artistic media installations including Weather Tower (2008), an installation that turned weather data into abstract forms on the building. The tower had been unused since 2008 apart from a persistent 10-minute animation that ran sporadically to demonstrate the system's capabilities. The disuse of the Dexia Tower's LED façade coincided with the dramatic collapse of Dexia Group in 2011, and the bank has since decided that the changes in the public's perception of the company no longer allowed for the kinds of displays they had once sponsored and supported. The Media Screen Flagey Square, also in Brussels, presents another example of contextual friction caused by media architecture. The $12 \mathrm{~m}^{2}$ screen had been vandalized with paint and its supply wires were set on fire on a number of occasions as a result of it being perceived as an unwanted "commercial invasion" for displaying ads for luxury retailers in a low-income area of the city. This represented a failure in contextual sensitivity with respect to the content and environment of the media façade, resulting in a form of community-initiated dissent or counter-monumentality. 


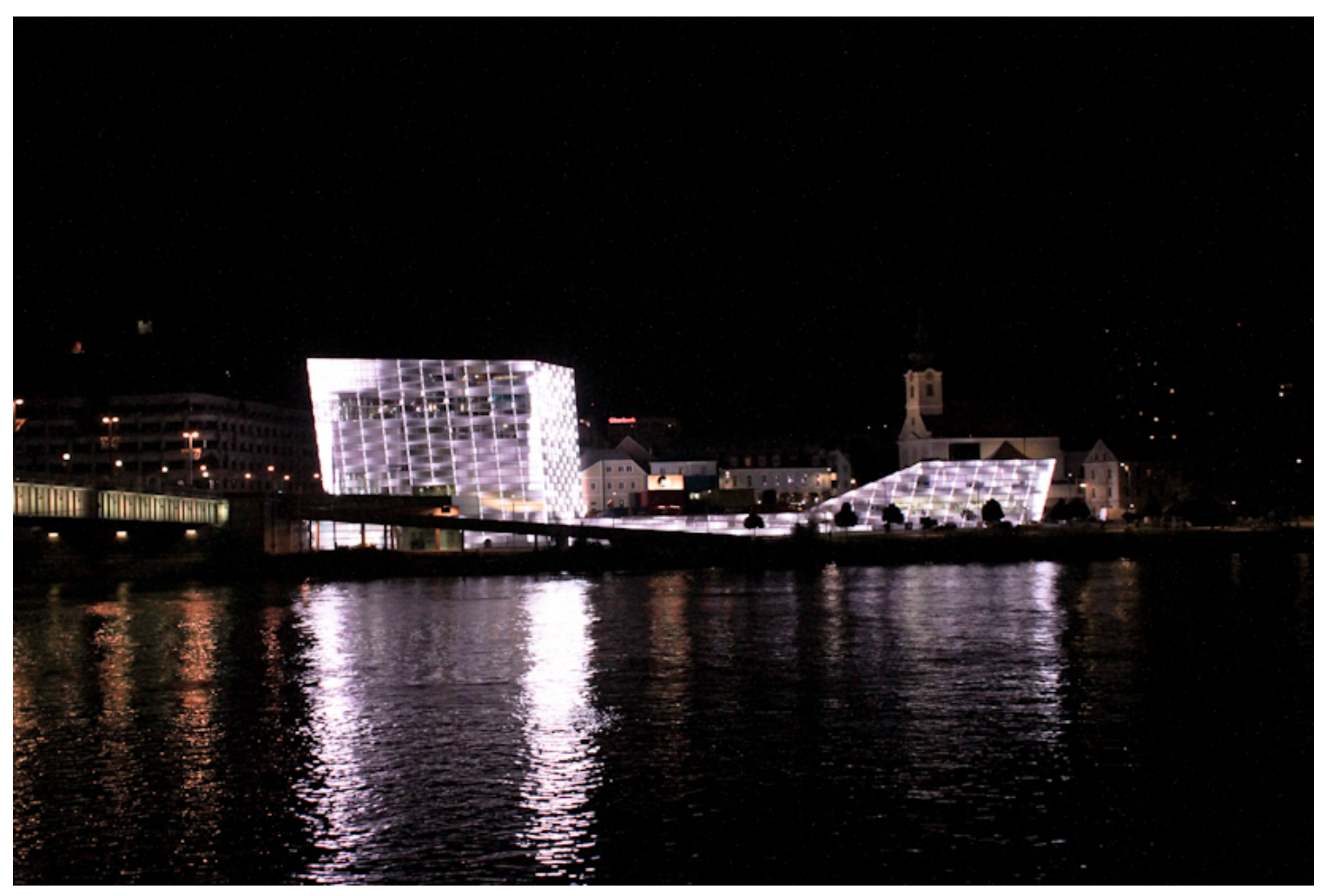

Figure 30. Onur Sönmez and Tamer Aslan, NOX (2013), Ars Electronica Center, Linz.

One particular example of a media façade that has struck a balance between environment, content, and carrier is the Ars Electronica Center in Linz, Austria (see Figure 30). The building, consisting of 1085 3'x1' back-lit glass panels, has consistently been used for artistic experimentation since 2009. Part of the official mandate of the Ars Electronica Center involves "testing ways in which we might be interacting and communicating with our surroundings and other human beings in the very near future, and getting an impression of what these changes will mean for us and our society" (Ars Electronica 2015). As such, the Center deliberately and consistently seeks to develop its façade as a sustainable, coordinated exhibition space for experimental public data visualizations. The Ars Electronica Center thus occupies a unique space in the massive media world in that it 
supports access to the façade and its surrounding space of exhibition and interaction through curation and actively develops an audience for its presentations. One of Ars Electronica's most recent projects, NOX, consisted of a pull switch suspended in the middle of a viewing area in front of the façade that, when activated, turned all of the pixels on the building on or off like a lamp. The simplicity of the installation posed a simple, yet crucially relevant and brave conceptual question about massive media: are such façades seen as purely aesthetic embellishments and superficial gadgets layered over architecture, or do they signal an important shift in our urban fabric that seeks to make buildings more performative and expressive of the actions and desires of citizens? Can they be designed and programmed to be more accessible and representative of the wishes of a certain public? It made the façade itself into an urban issue central to the emergence of expressive, programmable architecture. The Ars Electronic Center, with installations like NOX, point to the promises and pitfalls of digital communication (and communion), and the possibility for massive media to engender and engage community and debate through public data visualizations that are situated, informative, and functional.

\section{The Empire State Building as Public Data Visualization}

While the previous examples are instructive of the ways we might evaluate lowresolution media façades and public data visualizations, what particular challenges and possibilities for public sociality and monumentality exist for a massive media building of this scale and stature of the Empire State Building? In the following section, I describe the Empire State Building's current lighting program in detail as well as its associated traces on 
Instagram and Twitter and use this information to evaluate the role of the Empire State Building against the criteria set out in the previous section: how is it situated, informative, and functional, and how does it manage aspects of environment, context, and carrier? I also consider how these changes in architectural lighting affect monumentality, public space, and the public sphere.

An analysis of the data from the @EmpireLights Twitter feed, an unofficial account announcing daily lighting event information culled from the Empire State Building's official site, ${ }^{7}$ shows that since 2009 , when the first LED lighting system was installed (later fully upgraded in 2012), the lights have represented holidays, charitable causes, sporting events, commercial promotions, private lighting events, and other occasions (such as memorials or parades), while also defaulting back to "all tiers white" for "no occasion" in between. The following chart (see Figure 31) presents a breakdown of these categorized uses based on a total of 1454 tweets collected from the account between 2009 and $2013 .{ }^{8}$

\footnotetext{
${ }^{7}$ The official Empire State Building Twitter feed, @EmpireStateBldg, also tweets lighting information nightly, while interspersing these tweets with non-lighting related information about events and activities at the venue. @EmpireLights was selected as it reports exclusively on the lighting scheme of the tower every night.

${ }^{8}$ The R statistical analysis program, along with the twitteR plugin, were used to gather historical tweet data from the @empirelights account. See http://www.r-project.org/ and http://cran.r-project.org/web/packages/twitteR/index.html.
} 


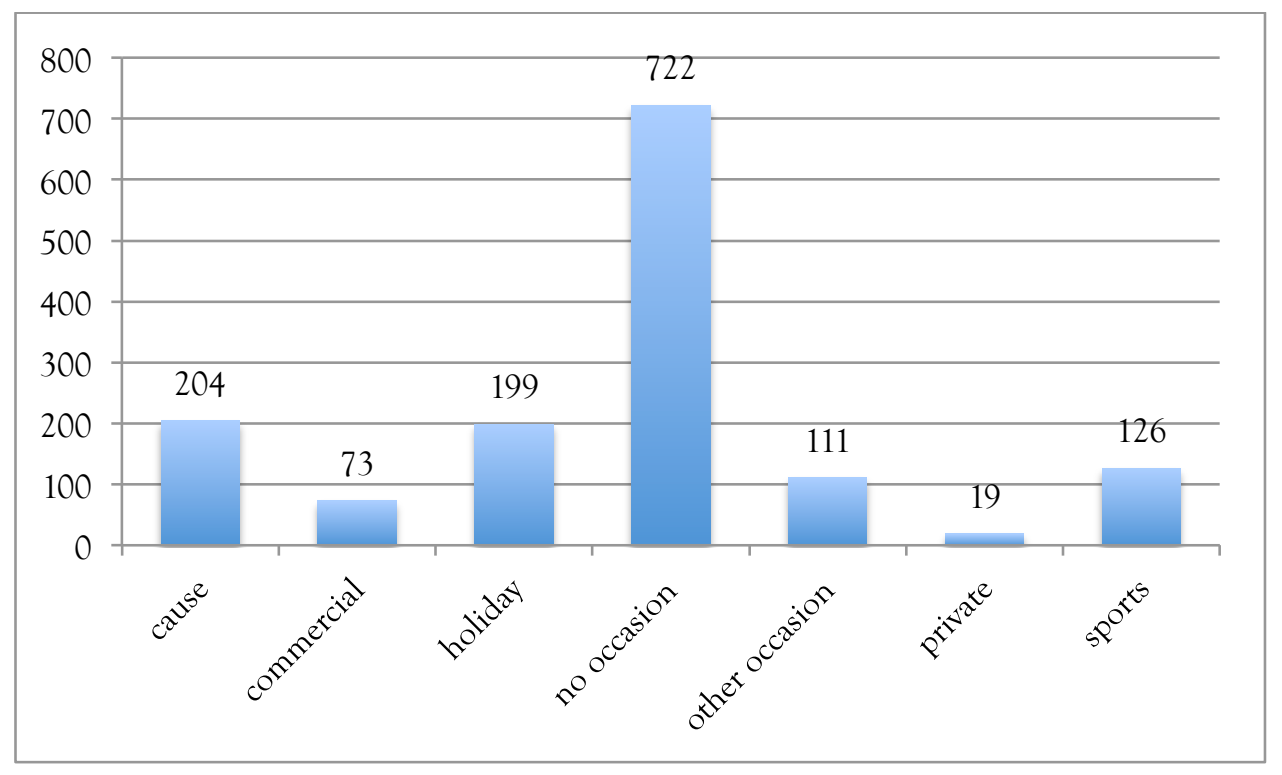

Figure 31. Categorized uses of the Empire State Building's lights, 2009-2013.

Examples of lighting programs characterized as a "cause" include events and milestones of not-for-profit organizations such as "@boyscouts 100th \#Anniversary" (red, white, blue), and “Autism Awareness Month @autismspeaks” (all tiers blue). These represent $14 \%$ of the total uses. Commercial lighting events, representing $5 \%$ of the total, have included promotions for "Caribbean Week @ctotourism” (blue, green, yellow), and “@MammaMiaMusical's 10th anniversary” (blue, pink, white). Holiday lighting schemes, representing 14\% of the total, have included multiple nights of "\#Christmas" (red, green, green) and "\#Hanukkah" (blue, white, blue). Approximately 50\% of all lighting days have been "no occasion" (all white), although this has become less frequent over time. Other occasions have included "Mexican Independence Day @MCINY" (red, white, green), and "Family Day" (blue, red, blue), representing $8 \%$ of the total. Occasionally, the building's lights are used for private events or film shoots, although this represents less than $1 \%$ of total use since 2009. Finally, sporting events make up 9\% of the total lighting schemes, 
with events such as "\#SubwaySeries" baseball games illuminating half of the tower in "blue, white, blue" for the Yankees and the other half "blue, orange, blue" for the city's other baseball team, the New York Mets. In addition to the categories listed above, certain uses can fall under more than one category. For example, appeals to donate money to charity (@FoodBank4NYC) appear during Christmas and Thanksgiving lighting tweets.

Commercial and charitable causes can also be mixed: EsteeLauder's \#BreastCancer awareness campaign is one example (all tiers pink).

The Empire State Building has also included more dynamic and complex uses as a public data visualization. In addition to the public data visualization of the 2012 election, during a promotion for the 2014 Super Bowl, in the week leading up to the game, fans had the opportunity to pick which team's colours would appear each night by tweeting either "Broncos" or "Seahawks" to \#WhosGonnaWin (see Figure 32). The information about how to participate, widely distributed online and through various media outlets, formed both the impetus to participate in a massive public data visualization and the means by which its meaning could be disseminated. 


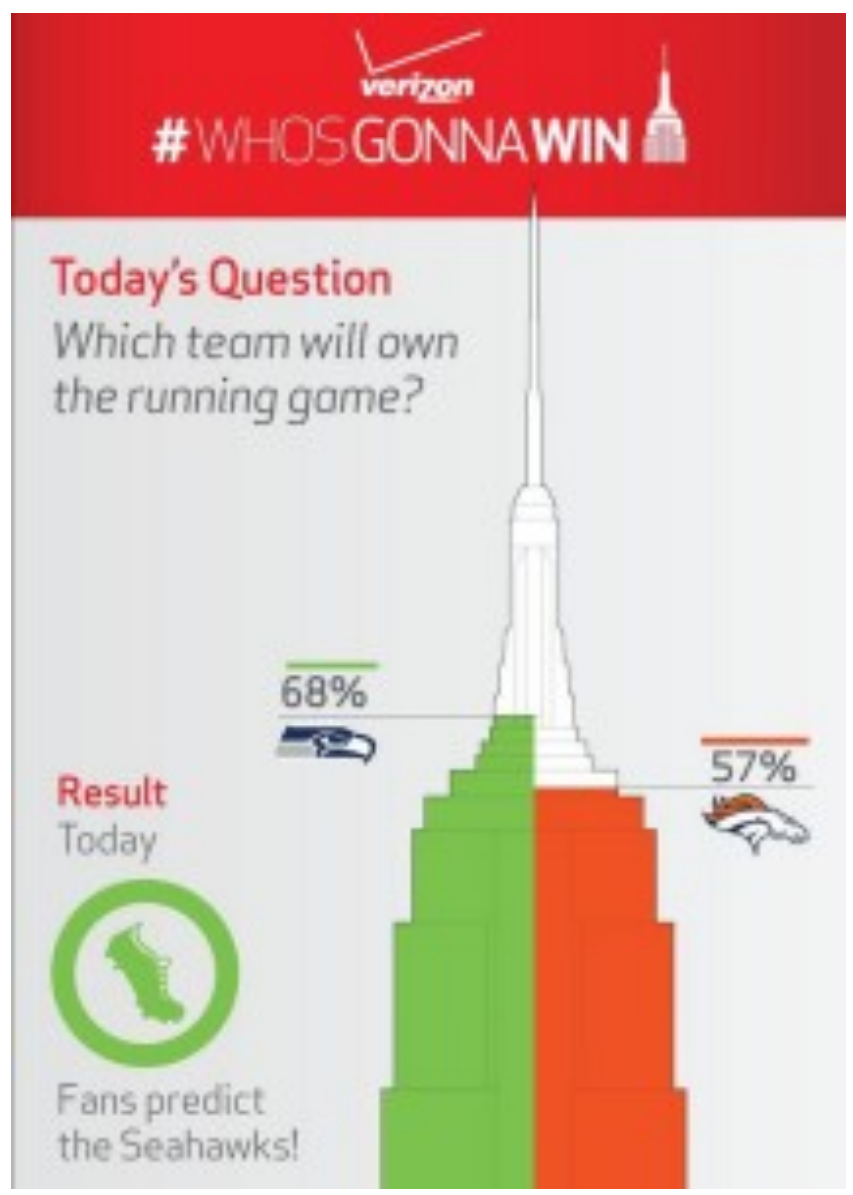

Figure 32. Verizon's \#WhosGonnaWin promotion for the 2014 Super Bowl.

Considering Vende Moere and Hill's criteria, an evaluation of \#WhosGonnaWin can be made based on the level with which it was situated, informative, and functional. In terms of this visualization being situated, it existed in the midst of the hype surrounding the week leading up to the Super Bowl in the city in which it was to be hosted, thus having a direct and immediate relationship to the local context. That said, the data that was acquired extended beyond the local context, incorporating information gathered form fans around the world. The visualization was informative, in that it created meaningful insights into the enthusiasm and support for either team. Finally, it was functional in that it was able 
to reach a large audience by virtue of the scale of the building in Manhattan and through the provision of online participation and promotion. This particular example demonstrates one of the roles massive media, in its ambivalence and contingency, can play: as an urban spectacle and as an advanced site of advertisement that can anchor a promotional campaign.

Furthermore, while the visualizations, causes, and events featured on the Empire State Building reflect issues that are closely relevant to the social, cultural, and commercial reality in its vicinity, the building still requires and feeds off of the support of social media to reach its audience, that is, to be truly situated. For example, there is no difference whatsoever between the lights (blue, white, blue) used to celebrate Hanukkah, the Yankees, Columbia University commencement, or the $200^{\text {th }}$ Anniversary of Argentina, except as indicated online. In this sense, the building must be considered to be situated through the hybrid relationality of on- and offline spaces. From this, we might conclude that the environment of a building such as the Empire State Building, and its associated lighting and social media, help to transfer meaning and power between on an offline spaces, just as monuments have transferred meaning and power with their surroundings in the past (Rowlands and Tilley 2006).

By linking into a social network, particularly forums such as Twitter, Facebook, and Instagram, the Empire State Building integrates its media façade into the flows and associations of online discourse, expanding the reach, visibility, and function of the building as a public data visualization and monument, and produces a public sphere of experience and ritual that bridges the gap between on- and offline space. It engages with its 
environment, that is, its immediate vicinity in an expanded sense, one that includes online spaces, extends its content, that is, the information displayed via the LED lighting system, to online environments, and fulfills an augmented role as carrier in that it piggybacks on networks to support its broadcast, shaping the interpretation of the content it displays beyond its immediate physical surroundings. It demonstrates the role that massive media plays in enabling a hybrid or relational space (De Souza e Silva 2006, McQuire 2008) through the contingency and ambivalence introduced into public space via supporting communication networks and ubiquitous mobile devices. It also exemplifies the concept of composite dispositif, combining semi-coordinated standardized environments of spectatorship such as urban space and the smartphone to co-constitute a relational experience (Verhoeff 2012). Furthermore, massive media like the Empire State Building transforms the role of the monument, enabling more dynamic, participatory, transverse, and participatory rituals that can be connected to a public sphere that exhibits new "spatial, territorial and geopolitical parameters" (Hansen 1993), and that contends with competing discourses of art, politics, and commerce.

To delve more deeply into this phenomenon, I analyze some examples from Twitter and Instagram conversations related to the latest incarnation of the Empire State Building's lights. They demonstrate how relationships between an expanded environmentcarrier-content context are stimulated and maintained through the new monumental and relational spaces created by public data visualizations and associated media: how massive media merges the logic of screen media and the monument to engage mass culture, collective memory, and communal experience. 


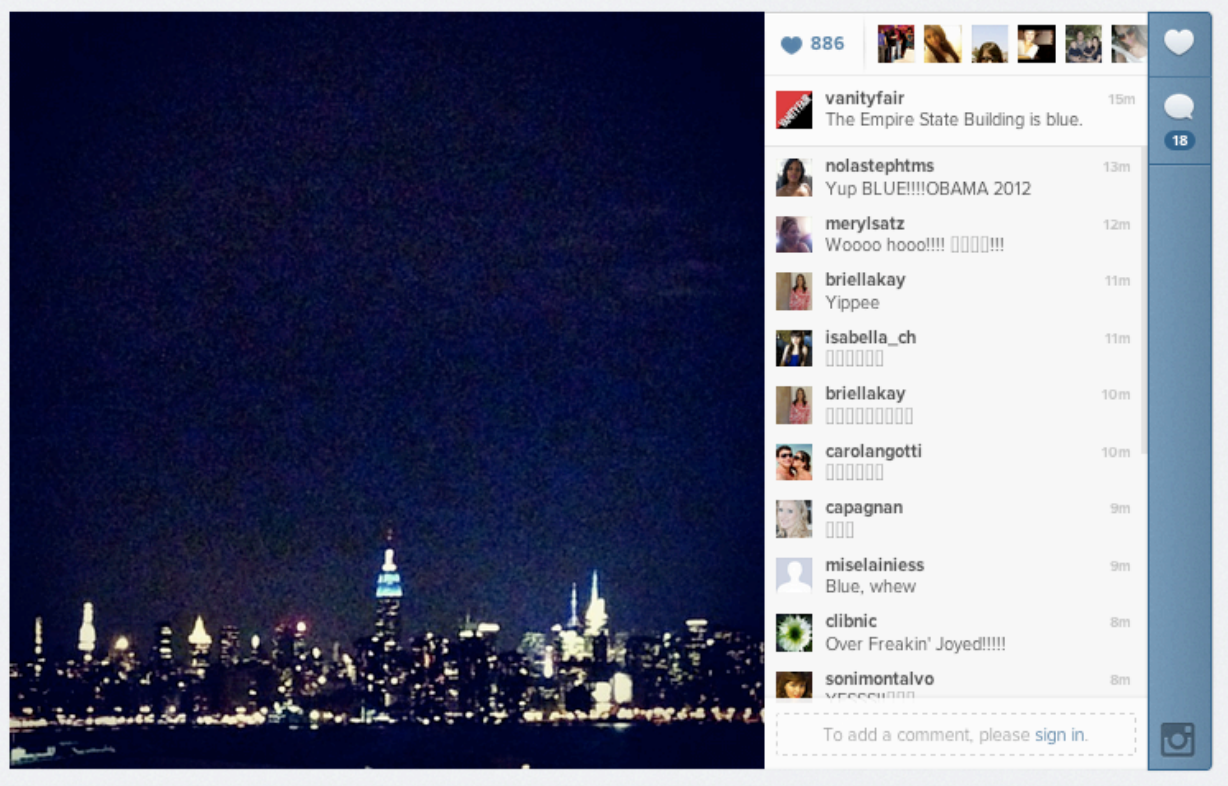

Figure 33. Empire State Building with blue lights indicating President Obama's victory in 2012 election, '@vanitfair', 2012, Instagram.

The first example is from the @vanityfair Instagram account (see Figure 33). It depicts the Empire State Building in the middle of the New York City skyline, lit in blue to indicate the victory of the democratic candidate, Barack Obama, in the 2012 presidential election. The text provided by @vanityfair simply states "The Empire State Building is blue" which means that those who responded must have heard about the meaning of the lights beforehand through another media channel (Internet, Twitter, television, radio), word of mouth, or would have inferred its meaning from knowing it was election night or from other comments on the post. Most of the comments are positive, with the open rectangular boxes in the image standing in for heart and hand-clap "emojis" that did not register on the web interface of Instagram. The comments here range from elation to relief; 
also, we see that 886 people liked this post, and a total of 18 people commented on it, making it visible to their own followers in addition to those who follow the @ vanityfair account. For a brief moment, until this post was buried under subsequent posts vying for attention and screen space, this composite of light, social media, and monumental space took on an intensity and an emotional resonance as well as an associated corporate value for entities such as Vanity Fair, the Empire State Building itself, and New York City.

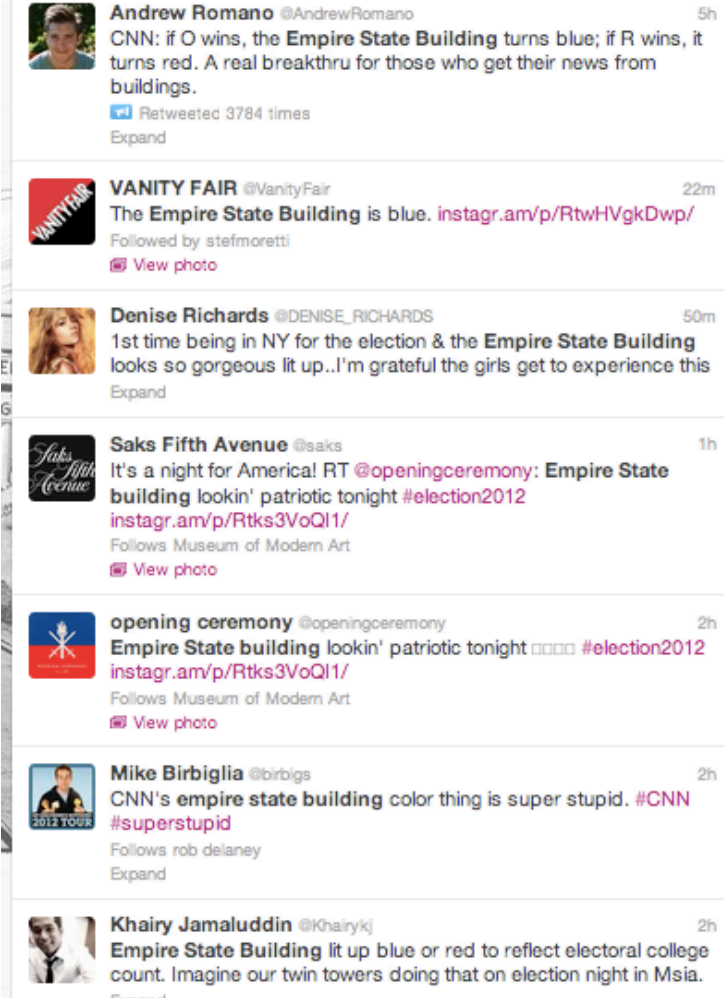

Figure 34. Selected Twitter results for "empire state building" during 2012 election.

On Twitter, at roughly the same time as the Instagram post, a sample of tweets containing the term "empire state building" provided a glimpse into how the election and the associated lighting event on the tower were discussed online (see Figure 34). Responses 
ranged from gratitude (from celebrity Denise Richards) to a tweet from Vanity Fair linking us back into the Instagram post discussed above. There was ridicule ("CNN's empire state building color thing is super stupid. \#CNN \#superstupid”), and even international envy ("Imagine our twin towers doing that on election night in [Malaysia]").
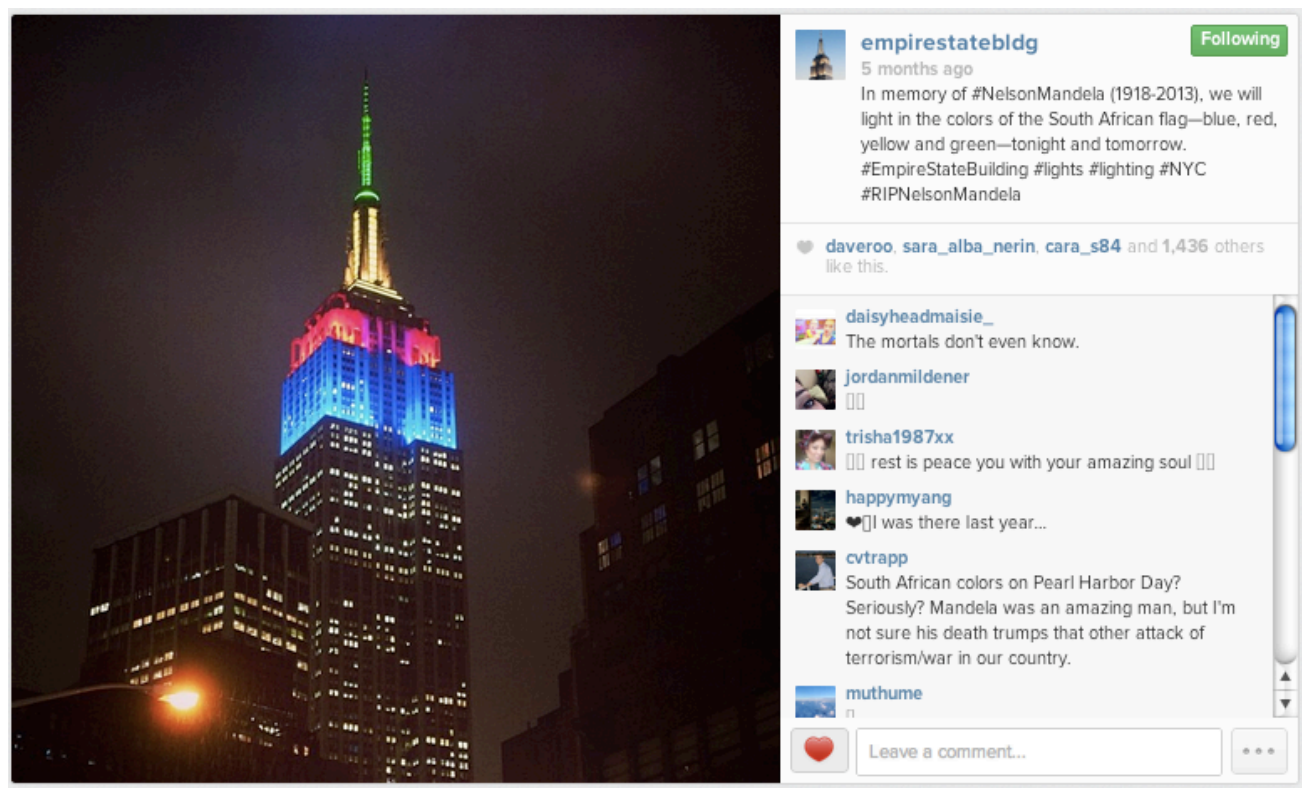

Figure 35. Nelson Mandela tribute on '@empirestatebldg', 2013, Instagram.

One final example involves a lighting configuration honouring South African political figure Nelson Mandela shortly after his death on December 5, 2013. That night, the tower was lit in the colours of the South African flag (see Figure 35). Most of the associated comments were positive and supportive, yet traces of dissent were also present. As Instagram user cvtrapp writes, "South African colors on Pearl Harbor Day? Seriously? Mandela was an amazing man, but I'm not sure his death trumps that other attack of terrorism/war in our country." This comment shows how the signifying power of the 
building and its lights, combined with the public function of its visibility, on- and offline, and the facilitation of feedback, can furnish transverse and asynchronous micro-publics of discussion, association, and debate within an expanded sense of carrier, content, and environment. Temporary nodes of intensity within increasingly contingent, networked spaces are created that extend the way that buildings like The Empire State Building can be considered to be situated, informative, and functional. The building becomes situated through a matrix of on- and offline references (the status of the building as an icon, and its online status/followers), informative, by broadcasting information about causes and events through multiple, connected channels, and functional, by generating and facilitating communal, habitual communication and attention for the building and, from time to time, its sponsors.

Despite this, there are limits to its ability to function as a public data visualization. One of the key concerns with all media forms-and media architecture is no exception-is access and moderation. While the Twitter, Instagram, and Facebook feeds related to the Empire State Building remain relatively unmoderated, protocols and procedures for determining what gets commemorated and represented by the lights remains highly controlled by the building's management. The Empire State Building's gatekeeping structures ${ }^{9}$ must balance the building's role as private entity and public icon carefully. While the building does entertain lighting requests, management clearly states that the building is privately owned and thus has its own selection and review policies. These policies include maintaining full discretion over selection and denying any requests for

\footnotetext{
${ }^{9}$ See the "lighting partner" application for the Empire State Building http://www.esbnyc.com/pr-pop-culture/tower-lighting-request.
} 
political figures and campaigns, religious figures, organizations, and holidays outside of the holidays they sanction (Easter, Eid al Fitr, Hanukah, and Christmas). That said, exceptions are made, seemingly arbitrarily, in instances such as the Nelson Mandela memorial lighting scheme and the presidential election coverage, to accommodate sponsors and popular sentiment. Examples such as \#WhosGonnaWin, where control over the lighting scheme is given to masses of sports fans willing to vote for turning the building the colour of their preferred team show that in the instances that access is (provisionally) granted to the public, it is ultimately brokered by the sponsor, Verizon, and limited to two choices whose referent has been predetermined. The public sphere that it substantiates in this instance is severely limited and does not foster meaningful dissensus and debate but channels this energy into drawing more attention to a sporting (and advertising) spectacle. While it does reflect a "new monumentality" in that it embodies a "collective emotional event" that attempts to allow people to play an important role in the unification of an architectural background, a population, and their shared symbols (Mumford 2000, 568) through its dynamic display, it also captures said elements in order to sell more phone plans for its sponsor and fuel the hype for a mass spectacle. Similarly, in the CNN election example, the building's lights are made to be dynamically responsive, but the choice of the lighting program is ultimately out of the hands of the public. While election information does provide an informative function, the participation afforded to the citizen by the public visualization is merely a token and does not (and cannot) reflect the direct result of an individual's participation. In both examples, the space of debate and dissensus is limited to the Twitter sphere, and while the powerful and highly visible building draws attention to 
this debate, it remains limited to a small, fragmented reception by those sifting through a stream of comments online.

That said, even this diluted form of interaction with buildings allows for the formulation of new kinds of publics and rituals that merge the embodied experience of the urban with "disembodied" online experience. Where publics are sites of discursive contestation circumscribed by the spatial and technological means that enable them, massive media can be seen to merge the originary public of concentrated visibility and assembly with more contemporary, fragmented, ephemeral groups. Massive media functions through the immediate visibility and feedback of a body politic with the fragmented, asynrchonous feedback of a transversal public that is made apparent through various platforms and protocols of networked communication. The result is a "(re)embodiment through technics" (Hansen 2006, 95) of a public which creates a new connection and a new register of "being together," where those present at the physical site of massive media are connected through networked media to those watching and participating from afar. The practices described in the examples above denote new rituals of monumentality (liking, voting, debating, clicking, tracking), commemoration (ephemeral repetition of historical remembrance), awareness (ambient representations that can increase the legibility of the city), and of course commercialism (through syncretic association of iconic buildings and brands, as well as the direction of audiences and consumers to various sponsors through the recentering qualities of participation).

Essentially, a building like the Empire State Building, as a site for public data visualization and a massive media monument, collects on- and offline audiences and their 
associated techno-social capabilities, allowing for a diversity of discourses associated with buildings that did not previously exist. It does so through the effect of making the building either directly or indirectly representative of a collection of individual impulses or by its availability for temporary recoding and appropriation through associated media. The city, regardless of the discourses associated with it via massive media, becomes more like a shared resource, a critical site for the formation, moderation, commercialization, and critique of what we might consider public debate and civic identification today. It becomes at once more communicative, communal, and commercial. Sutured as we are to the means to decode and interact with these buildings (via mobile ubiquitous media), with massive media we become avant-garde readers, writers, and consumers in a contingent, ambivalent city that creates powerful spatial and emotional associations and intensities.

\section{Creation-As-Research: Socially-Engaged Public Data Visualization}

The development of the Empire State Building's lighting program for purposes of commerce and collectivity raises some important questions about the practice of public data visualization, monumentality, and massive media. Namely, how can the use of similar façades best translate and direct our current dynamic reality towards artistic, political, and social ends? How can a low-resolution media façade better engage a democratized urbanism, engender debate, or elicit emotion? I have pursued these question through the creation of two large-scale, participatory public data visualizations. In this section, I describe these projects and evaluate their role as public data visualizations. 


\section{E-TOWER}

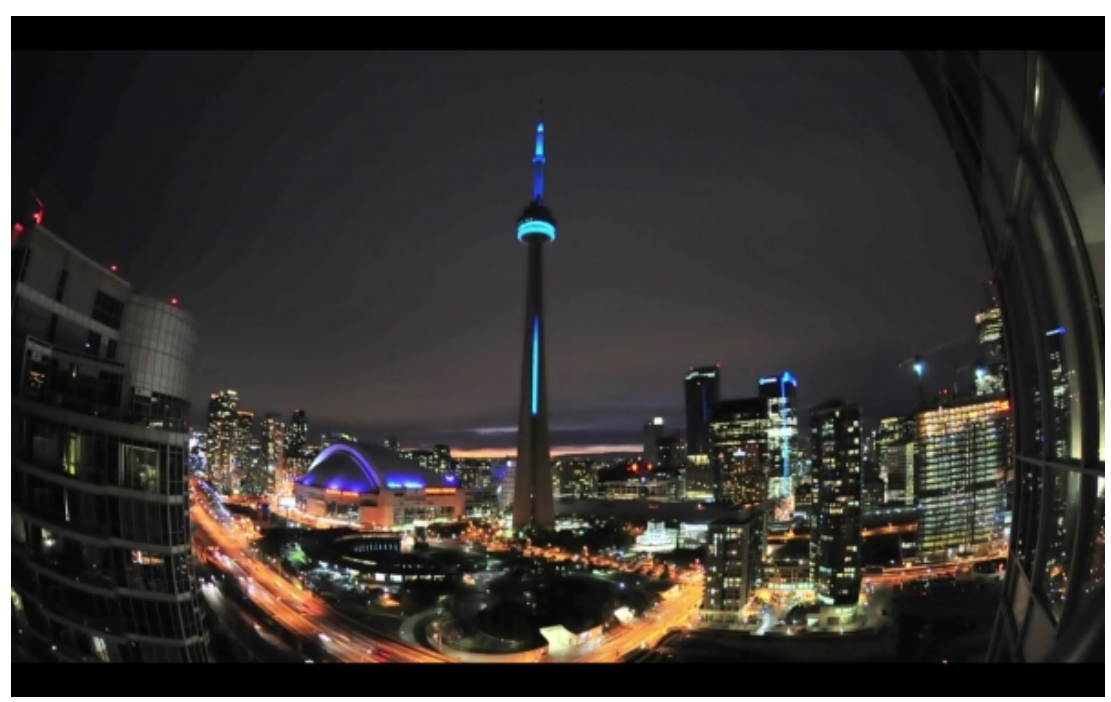

Figure 36. Dave Colangelo and Patricio Davila, E-TOWER (2010), CN Tower, Toronto.

E-TOWER (2010), completed with collaborator Patricio Davila, ${ }^{10}$ was an interactive cell-phone based installation with one of the tallest and most visible low-resolution LED façades in the world, Toronto's CN Tower (see Figure 36). The CN Tower, a federally owned and controlled building initially built by CN Rail in 1976 to boost television and radio broadcast signals and to create a landmark for the growing city, was outfitted with a computer controlled LED lighting system in 2007 and has since followed a similar trajectory as the Empire State Building, commemorating holidays, special events, memorials, and special causes, while otherwise defaulting to its signature red colour to

\footnotetext{
${ }^{10}$ Patricio Davila and I jointly collaborated on E-TOWER (2010). For this project, I was responsible for the initial concept and proposal that was accepted by the Nuit Blanche 2010 committee in Toronto. Together, Davila and I developed the concept further and we secured permission from the CN Tower and sponsorship from Rogers Communications to pay for production costs. Davila and I were both involved in developing the final concept as well as in production meetings with Rogers, Nuit Blanche, and the CN Tower. We worked iteratively throughout, with Davila taking on most of the programming duties while I assumed most of the production duties including web design. We received in-kind assistance from graphic designers Berkeley Poole (flyers) and Jamie Webster (tower animations), as well as in-kind documentation support from Udo Dengler (time-lapse video) and Sarah Lasch (promotional video).
} 
symbolize Canada. It broadcasts this information via Facebook, Twitter, and Instagram feeds. The CN Tower is an example of massive media, merging the logic of the screen with the logic of the monument to create a hybrid, relational space of collective memory and identity with accelerated rituals of identification and participation.

After a period of negotiation with the tower's management and careful vetting and revision of our concept (it could not be seen as politically or commercially driven as the federally owned CN Tower is technically a government building), as well as securing technical support and sponsorship from one of Canada's largest telecommunication companies, Rogers Communications, we were granted access to the tower's lighting system to present the work during Nuit Blanche 2010, an all-night art party held every year in Toronto. From sundown to sunrise, E-TOWER visualized the collective energy of the city on the most visible symbol of the city (carrier) by asking participants to text the word 'energy' to a specific number, making the lights on the tower grow faster and glow brighter. ${ }^{11}$ Throughout the night, individuals and groups participated by sending their "energy" to the tower (content), creating links across physical space, in proximity and at a distance, sutured together by mobile phone technology and the tower as a shared representational and communicative beacon (environment). E-TOWER allowed participants in the city (situated) to communicate with each other through a visualization combining data, architecture, and urban space. By using the $\mathrm{CN}$ Tower as the central transfer point for the city's participation, people's actions were mapped onto a powerful civic symbol, engaging a new register of monumentality that was immediate and representative.

\footnotetext{
${ }^{11}$ See http://www.etower.ca/ for documentation of the work.
} 
Furthermore, E-TOWER coordinated the diverse and distributed screens of participants with the tower as a central visualization, using the visibility and centrality of the tower and the ubiquity of phones to create an attractive experience and a specific gathering point for identification and participation-a composite dispositif. It was able to create and make collectivity and connection massively attractive and apparent (informative and functional), dramatizing architecture and the city by visualizing the data of participation.

That said, and despite the tower's omnipresence and visibility, there were significant challenges in developing an audience for this work. The biggest challenge came from the fact that on the night of Nuit Blanche, although there were almost one million people in attendance (Bradshaw 2010), there were also approximately 300 other projects vying for attention. Not only was the audience peripatetic and geographically dispersed, they were also being acted upon by the sights and sounds of other dispositives. The work needed to somehow capture and hold the city's thinly-sliced attention. This brings to mind the concerns of Crary and Virilio, who both warn against the dangers of distraction inherent in the proliferation of technology and moving image in public spaces with no apparent end game. The experience made us realize that single-night experiments in the context of a city-wide art party may not be the best way to construct and test new registers of monumentality through massive media. Longer exposures and deeper involvement with a public through interactive projects that last weeks, if not months, could serve to better develop audiences and help to determine what they see as the best use of these new assemblages of light, architecture, and data. 
Another challenge we faced came early in the process. As noted above, our concept changed throughout the course of project development due to pressures from the $\mathrm{CN}$ Tower's management. Initially, we had hoped to reflect the relative emotional states of participants on the tower with representative colours by asking them to send us a message with one of five feelings (happy, sad, bored, excited, and angry) and creating a framework for their visualization. Before developing this concept further, we were told by the management of the CN Tower that this concept would not be acceptable. They were concerned that the tower might be associated with negative emotions. What this shows is a politics of aesthetics in action in which the carrier of the public data visualization, in this case the $\mathrm{CN}$ Tower, sought to pre-emptively protect their reputation by censoring our plan for the public display of emotion on the tower. Clearly, there are limits to what can and cannot be said via public data visualization, and in the public sphere in general, and these limits are imposed by the owners of the means of communication and representation. ETOWER, although presented in a form that was shaped by these constraints, allowed us to meet, experience, and understand the limits to expression, particularly in the context of such a highly visible and highly regarded symbol of the city. 


\section{In The Air, Tonight}

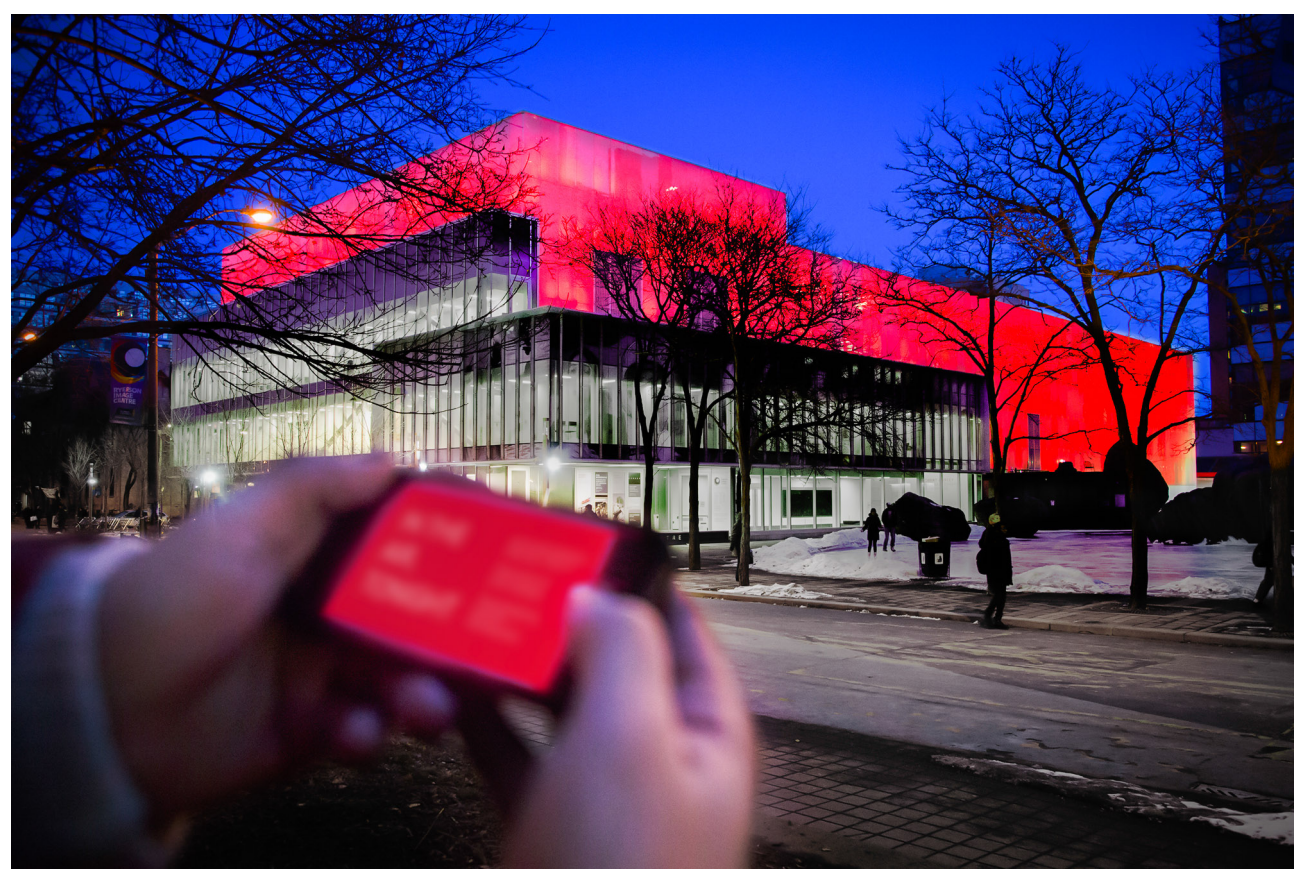

Figure 37. Dave Colangelo and Patricio Davila, In The Air, Tonight (2014). Ryerson Image Arts Building, Toronto.

For our next project, ${ }^{12}$ we partnered with the Ryerson Image Arts Building, a newly renovated building with a computer controlled low-resolution LED façade on the campus of Ryerson University in downtown Toronto. Although the building was owned and operated by the university and had yet to display content outside of ambient pre-set patterns and school colours, the administration of the building allowed us to test some new

\footnotetext{
${ }^{12}$ In The Air, Tonight (2014-) was a collaboration between myself Patricio Davila. Initial conceptualization and negotiations with the administration of the Ryerson Image Arts Building LED façade were made by myself, Jon Friis, and Takin Aghdashloo in 2011. After Friis and Aghdashloo left the project, Davila joined and we jointly refined the concept for the project, raised funds, and put together a team to complete the project. I was involved in initial programming, building animation design, and computer modelling. Davila hired and directed Robert Tu in completing the programming for the back-end of the installation (php and Processing) while Maggie Chan was hired to complete the building animations and produce documentation of the month-long installation. The team shared various production duties including refining the concept, installing a remote weather station on the roof of the building, installing a webcam, producing promotional materials, moderating the Twitter feed, and public relations planning and execution. In-kind consultation and support was provided by street nurse Cathy Crowe, public relations analysts Claire Larocca and Alen Sadeh, professor and media artist David Bouchard, and the staff at the Ryerson Image Arts Building. For the second iteration of the project, David Schnitmann replaced Robert Tu to provide additional technical support.
} 
possibilities as they were not beholden to sponsors, government mandates, or other corporate stakeholders. This access and freedom from moderation allowed us to present In The Air, Tonight (2014-) (see Figure 37). ${ }^{13}$ For one month, during one of the coldest winters on record in Toronto, the LED façade of the Ryerson Image Arts Building (carrier) was animated with a blue wave representing wind speed and direction while an intermittent red pulse was triggered by fluctuations in the use of the hashtag \#homelessness on Twitter (content). By visiting intheairtonight.org people could read and retweet messages from our Twitter feed (@itat2014) or compose their own messages. Every message with the hashtag \#homelessness amplified the issue online and contributed to a colour change on the building (environment). Our goal with this project was to foreground a pressing social and civic issue through networks and architecture, negotiating access to both, and providing an interface that allowed people to engage with and contribute to amplifying an area of common concern-to create a participatory public sphere around a specific issue through massive media. A key component of this project was to seek out experts in the field to help populate and seed the Twitter feed for the duration of the installation to enrich the public sphere we were attempting to create and amplify. Another main component was making connections with drop-in centres and homeless shelters in the area around the building that provided computer and internet access to homeless individuals in order to allow those directly affected by this issue to participate in the project.

While many people engaged with the project online through Twitter and through media outlets that had written about the installation, participation and awareness of the

\footnotetext{
${ }^{13}$ See http://davecolangelo.com/project/in-the-air-tonight/ for documentation of the work.
} 
installation at the physical site was limited. What the project lacked was a strong referent or associated physical medium on-site in order to make the data visualization more selfevident; or, in other words, to make it more situated. Unlike the NOX project at Ars Electronica, where a simple pull evoked quite clearly the function and purpose of pulling it, the lights on the Ryerson Image Arts Building did not self-evidently refer to the information about homelessness that they were linked to. And, while the building stood at the centre of the highest concentration of drop-in centres and shelters in the city, this much was not clear by simply looking at the building. That said, In The Air, Tonight was informative in that it was clearly responsive to tweets, yet was not necessarily insightful, in the sense that it did not easily allow onlookers to create meaningful insights about the problem of homelessness. Instead, it allowed them to do so only if they engaged in the online component of the work and followed links and tweets served up on the interface. Finally, In The Air, Tonight, could be said to be somewhat functional in that it was aesthetically pleasing and was persuasive for those that had deciphered its meaning. At the same time, the project was functional in that it was able to reach a larger audience by tapping into other associated media: it was able to capture media attention (radio, blogs) from outlets that would not necessarily be concerned with poverty and homelessness (see Sokol 2014 and CBC 2014) and did increase the prevalence of the hashtag "homelessness" online, making the issue more visible on a number of fronts. As such it extended the environment-carrier-content meshwork across on- and offline space, extending the reach and impact of the project and the building. It also led to many connections between experts and activists in the field that became aware of one another via the Twitter stream. 
In short, the low-resolution media façade event was the impetus for the formation of diverse, temporary publics and publicity through the creation of a composite dispositif centred upon a spectacular urban display.

In comparing our work on the CN Tower and the Ryerson Image Arts Building to the display program of the Empire State Building, it is clear that the creative and critical use of massive media and public data visualizations lies with the mandate of the host organization and its openness to artistic control and experimentation. As such, our role as (massive) media artists seemed to depend greatly upon our ability to secure access to media architecture by convincing organizations to allow non-commercial, exploratory alternatives to appear on their façades. Essentially, Davila and I became activists, with both E-TOWER and In The Air, Tonight, in our task to work towards gaining access to these expensive and highly guarded public displays to make them more public, that is, to open them up to wider participation, debate, and identification. Our role (and goal) was to furnish and amplify cooperation (in the case of E-TOWER) and productive dissensus (in the case of In The Air, Tonight) by gaining access to massive, programmable architectural surfaces in prominent urban settings and to create interfaces and rules of engagement to approximate more closely political positions that value the public representation and debate of translocal citizens.

Davila and I also took on an important role as curators of these spaces, in a sense, in our responsibility for appreciating and connecting with the context in which our work was being presented. In fact, the work very much emerged from a careful consideration of and negotiation with contextual factors, such as discussions with CN Tower management 
or the realization through consultation and research that the Ryerson Image Arts Building is situated amongst the highest concentration of drop-in-centres and homeless shelters in Toronto. We were also curators in the sense that we had to learn how to present our work on these highly complicated exhibition spaces with minimal support from the host institutions. To that end, technical and contextual challenges of producing massive media works, and the role that a curatorial organization might play in addressing these challenges, are discussed in the following chapter.

\section{Supermodernism}

Low-resolution programmable façades on buildings like the Empire State Building, the CN Tower, and the Ryerson Image Arts Building participate in an emerging and important practice of public data visualization that dramatizes architecture, makes data visible, and, at best, connects citizens to important civic issues. They create and support hybrid and relational spaces that activate narrative and associative potentials through their expressivity, networked digitality, and contingency. In these scenarios, the visibility and monumentality of architecture is important in that the power it yields can be harnessed and redirected through associated media on and off the building. Scale and physicality give a new shape, form, and context to data. At the same time, data can give a new shape, form, and context to architecture. Data becomes sensual and perceptible on a large scale (big data can be seen on a big scale), and distributed communication can appear more communal in publicly shared spaces coded as such. In this way, large, low-resolution programmable façades are a digital-era update to things like weather beacons and TV towers, except that 
they are exponentially more expressive, iterative, and communicative, especially through associated technologies and networks of ubiquitous social media.

To better historicize this phenomenon, programmable architectural media façades can be said to be suggestive of supermodernism, a movement in architecture that involves a new epoch of urban form characterized by buildings seen increasingly as communicative systems that prioritize direct, contextually relevant experience (Ibelings 2002) and thus seek to re-inject specificity and connection into physical environments where it has been seen to have been lost (Crary 1999, Virilio 1984). Supermodernism shifts the traditional notion of architectonics (Frampton 1983), a term that describes the degree to which the material composition and symbolic expression of a building reflects its history and context, to include a responsiveness to data flows in the "surrounding information environment" (Krajewski 2005, 2), making this data concrete through a spatial expression and the experience of data against the body in space. While others, such as Virilio (1984) and Augé (1995) have warned that technology has set architectonics adrift, "exil[ing] all of us from the terrestrial horizon" (Virilio 1984, 550), Ibelings (2002) and Krajewski (2005) focus instead on ways to take back the power of public space to encode meaning by suffusing it with data, and remain more optimistic about the relevance and potential of buildings that can translate or reflect our present dynamic reality through screens.

Apart from providing opportunities for communal commemoration, celebration, and augmenting the presentation of data through context and scale, as buildings such as the Empire State Building and the CN Tower demonstrate, public data visualizations can also play the important and necessary role of allowing data to surface symbolically and 
circulate technically in ways that are sensible at sites and scales befitting their effects and importance in contemporary life. While low-resolution architectural façades may, as Felicity Scott (2003) points out, "signify a proximity to technological advancement" (80) of a certain time (and thus provide an intrinsic value to city builders), just as TV towers once did, they do more than signify: they converse, because they have a public face, a façade that talks, and, to a degree, listens. This, in the end, is what makes them supermodern and worthy of future study; they are, as Ibelings (2002) points out, increasingly seen as important parts of communicative systems and are meeting places for people and things that embody and engender the coming together of mediated communication in its myriad forms.

\section{Conclusion: Temporary Intensities and Collective Conversations in Relational Space}

This chapter's case study of the Empire State Building's LED façade analyzed social media as an important associated technology of media façades, particularly those with lowresolution displays. Like large-scale public projections, these emerging media architecture experiences and practices must continue to be analyzed and critiqued through observation, analysis, and practice so that we might better understand their role and potential in society, and their role in commodifying and/or enhancing urban identities and issues.

The expanded capabilities of LED façades, coupled with an associated technological milieu which includes mobile ubiquitous media, ever expanding contextually relevant data sets, and social networking, allows for a more dynamic relationship between people, the city, and information through their superimpositions, montages, novel composite 
interfaces, and opportunities for post-production. Massive programmable low-resolution façades, such as the one atop the Empire State Building, code and recode space in their ever shifting messages and meanings, and allow people to associate (or disassociate) with the city in novel ways while also allowing data to surface through public data visualizations that are situated, informative, and functional. Buildings with media façades, more than large-scale public projection sites, thus become durable and dense transfer points for digital culture, as well as for the emotions and identification of diverse groups, reinvigorating mass cultural, communal, and collective experience and accelerating rituals of monumentality. By harnessing the direct participation of publics through social networking (a necessity of their typical inability to display text or images), the massive media of programmable low-resolution façades ushers in an era of supermodernity that connects architecture within an expanding composite dispositif of screen and non-screen experiences of spectatorship and participation. Low-resolution media façades occupy a central role in the formation of temporary intensities within an increasingly relational space characterized by ambivalence and contingency (McQuire 2008), extending the environment-carriercontent context of public data visualizations into hybrid on- and offline spaces. The intensities created by such assemblages can be directed towards various ends, which, depending on the conditions of ownership and control-perhaps the strongest determining factor in the use and outcome of these assemblages-vary from overtly commercial to artistic, experimental, and critical.

Like the massive media of large-scale public projection, low-resolution media façades can become semiotic resources for socially engaged practices and urban activism 
provided that the proper permissions are negotiated, audiences are sufficiently fostered and developed, and consideration is given to equitable representation through interface design and context sensitivity. Artists and designers engaged in a practice of massive media must see as their primary objective the furnishing and harnessing of technologies and points of access which include public space, architecture, various fixed and mobile sensors, and networks, to facilitate and amplify collective conversations around issues of common concern. 


\section{CHAPTER 4: Curating Massive Media}

\section{Introduction}

A shift in the experience and definition of urban space occurs as a city's social and spatial context changes with the addition of more expressive and connected digital public displays in the form of low-resolution media façades, urban screens, and public projection. In such a media city (McQuire 2008), feedback associated with urban structures through screens and devices large and small serve to reconstruct contemporary life, instituting new ways of being social and civic. Orientation becomes more contingent and ambiguous, blurring lines between presence and absence, the near and the far, leading to what McQuire calls "relational space” (48), a space defined less by pre-existing relationships of familiarity and solidity and more by temporary, ephemeral connections and impressions. Similarly, theorist De Souza e Silva (2006) describes this entanglement and imbrication of media and space as "hybrid space" (271). For both authors, the key understanding is that space, in addition to being socially constructed (Lefebvre 1991), is also constructed through technological lenses, filters, and devices. Crucially, De Souza e Silva argues that in the hybrid spaces of the media city, "every shift in the meaning of an interface requires a reconceptualization of the type of social relationships and spaces it mediates" (262). When buildings become screens that people can interact with, new pitfalls and possibilities emerge that require critical reflection and curatorial nuance.

As mentioned in the preceding chapters, the proliferation of screens and moving images in public space has been met at times with derision: seen as potent distractors, screens can create "formless fields of attraction" that distort the legibility of the urban 
environment, diminish sociality, and dehistoricize a place (Crary 1999), particularly if they are used as they most often are: as advertisements. Take, for example, Times Square in New York City, a site that represents the epitome of capitalist spectacle and comprises one of the earliest and densest sites of urban screens. A heavily mediatized environment for the distraction and attraction of peripatetic mobile spectators and passersby, Times Square is a relational space in which spectators can easily become spellbound by the perpetual and frenetic rush of images urging them to identify and consume branded objects and content: it is the very definition of spectacle (Debord 1995). Times Square demonstrates the power of its confounding and dazzling heavily-screened public address through the dominant discourses (and dollars) of the corporations it attracts and broadcasts.

That is not to say that highly commercial screens cannot be allied with other functions, namely critical discourse and artistic exhibition. In fact, since 2012, Times Square has been the site of an ongoing Midnight Moment instituted by the Times Square Advertising Coalition (TSAC) and Times Square Arts. During a Midnight Moment program, 15 of the largest screens in Times Square are coordinated to display a single artwork for three minutes. In the past, the program has featured works by artists such as Tracey Emin (see Figure 38), Yoko Ono, and Alfredo Jaar (Times Square Arts 2014). 


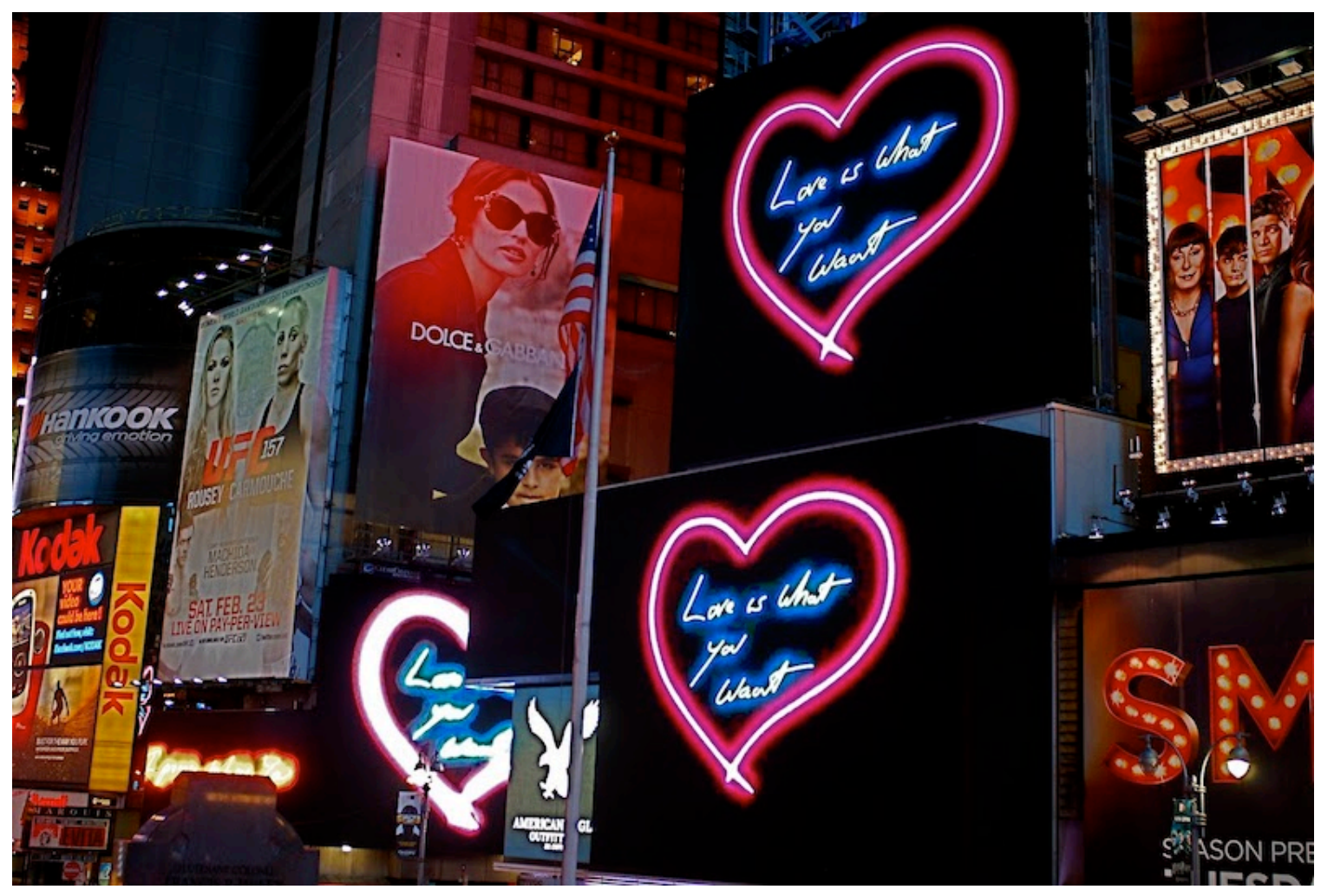

Figure 38. Tracey Emin, I Promise to Love You (2013), Times Square Arts Midnight Moment, New York. Photo: Ka-Man Tse @TSqArts, courtesy Times Square Arts.

In addition to the display of art on public advertising screens, there are a growing number of digital, outdoor, large-scale public displays that are dedicated to year-round noncommercial content, using the attractive power of scale and the screen (the logic of the monument and the logic of media) to capture audiences and compel them to view or even interact with artworks. Montreal's Quartier des spectacles is a network of permanent projection sites and lighting features in the city's core designed to showcase original content and integrated programming with various cultural festivals (McKim 2012). In the past, artists such as Rafael Lozano-Hemmer have animated the space with video- and lightbased installations. And while spaces like Quartier des spectacles do open seemingly greater possibilities for artistic innovation and audience development in the field of massive media 
than the sparse space and time afforded by commercialized sites like Times Square, Joel McKim (2012) argues that scenarios such as Quartier des spectacles do not guarantee artistic innovation and can just as easily contribute instead to the "general aestheticization" (135) and branding of the city for touristic and economic purposes. Quartier des spectacles, while presenting artworks designed for its many display sites, and never displaying ads, can still be seen as an advertisement for the "creative city" (Landry 2008) or an element of competitive differentiation. This, perhaps, is the central challenge for public art and its curation in general, particularly public art that requires high levels of capital investment and government permission to exist. How can the programing and curation of these sites deliver audiences to critical and creative encounters with art, the city, and each other within the challenges and constraints that come with producing complex and costly largescale public works? How can they avoid becoming mere advertisements for cities and for art in general?

\section{A Short History of Public Screen Practice}

Like well-trained judo wrestlers, artists and curators that have presented large-scale public displays have not necessarily confronted the power of monumental corporate adversaries directly so much as used their inertia to destabilize them, creating temporary publics for critique and contestation. Two interventions that established the genre of public screen practice include Jenny Holzer's Truisms (1981) in which the artist displayed phrases such as "PROTECT ME FROM WHAT I WANT" and "ABUSE OF POWER COMES AS NO SURPRISE” on Times Square’s Spectacolor screen, and Krzysztof 
Wodiczko's Astor Building (1984), a mammoth projection of a padlock on luxury condos in lower Manhattan. Public art critic Grant Kester notes that these works reclaim "the urban public sphere as a space in which differences of privilege and political power could be revealed and questioned rather than suppressed" (2006, 264). Works such as Holzer's and Wodiczko's use the large-scale projected image and architecture to create temporary publics that harness the power of visibility in the city and engage a "representative publicness" (Habermas 19819, 5) that is not always present in relatively stoic and unchanging architectural surfaces.

More coordinated and sustained efforts into the critique of the role that large urban displays play in the quality and construction of public life have come from the landmark curatorial organizations Artangel Trust and the Public Art Fund. Both organizations have targeted electronic billboards as particularly potent sites in the struggle for representation in the city. Like Negt and Kluge (Hansen 1993), they can be said to view "the media of industrial commercial publicity, in their most negative implications, as an inescapable horizon, and as the most advanced site of struggle over the organization of everyday experience which contextualizes all other sites" (211). Specifically, London-based Artangel Trust (1985-91) focused on the use of advertising media such as billboards and outdoor screens (Connoly 2013), while Public Art Fund, Inc., founded in New York City in 1972 , focused on the placement of public art in a variety of urban neighbourhoods and contexts, including Messages to the Public, initiated in 1982, which made use of the Spectacolor board at One Times Square (see Figure 39) to display artist shorts of about twenty seconds in length, twenty minutes apart, inserted between advertisements (Phillips 
1989). As Patricia Phillips argues, inserting art into the temporary contexts of large, luminous public displays can be subversive, introducing a productive ambiguity between the art moment and the advertisement. Phillips argues that situating art within the spectacle of advertisements can show how "public life has become emblematic not of what is shared by a constituency but of the restless, shifting differences that compose and enrich it" $(1989,331)$. Spectacle is part of life, so why avoid it? Why not confront it head on and allow difference to play out from within? The work of Artangel Trust, Public Art Fund, and artists such as Jenny Holzer inquire into the medium of large-scale urban screens scenarios as they are most commonly seen: as advertisements that are out of our reach, questioning their effects and exploring the limits of dissent and dissensus by disrupting the habitual flow of corporate address.

Of course, the exploration of such limits can be highly limited itself. In the case of Messages to the Public less than 2\% of the total screen time was given to art. As such, the work might be seen at best as urban acupuncture, contributing to the health of the contemporary public sphere by stimulating the otherwise stifled "openness, inclusiveness, multiplicity, heterogeneity, unpredictability, conflict, contradiction, and difference" (189) of the public sphere in a highly commercialized zone of address. 


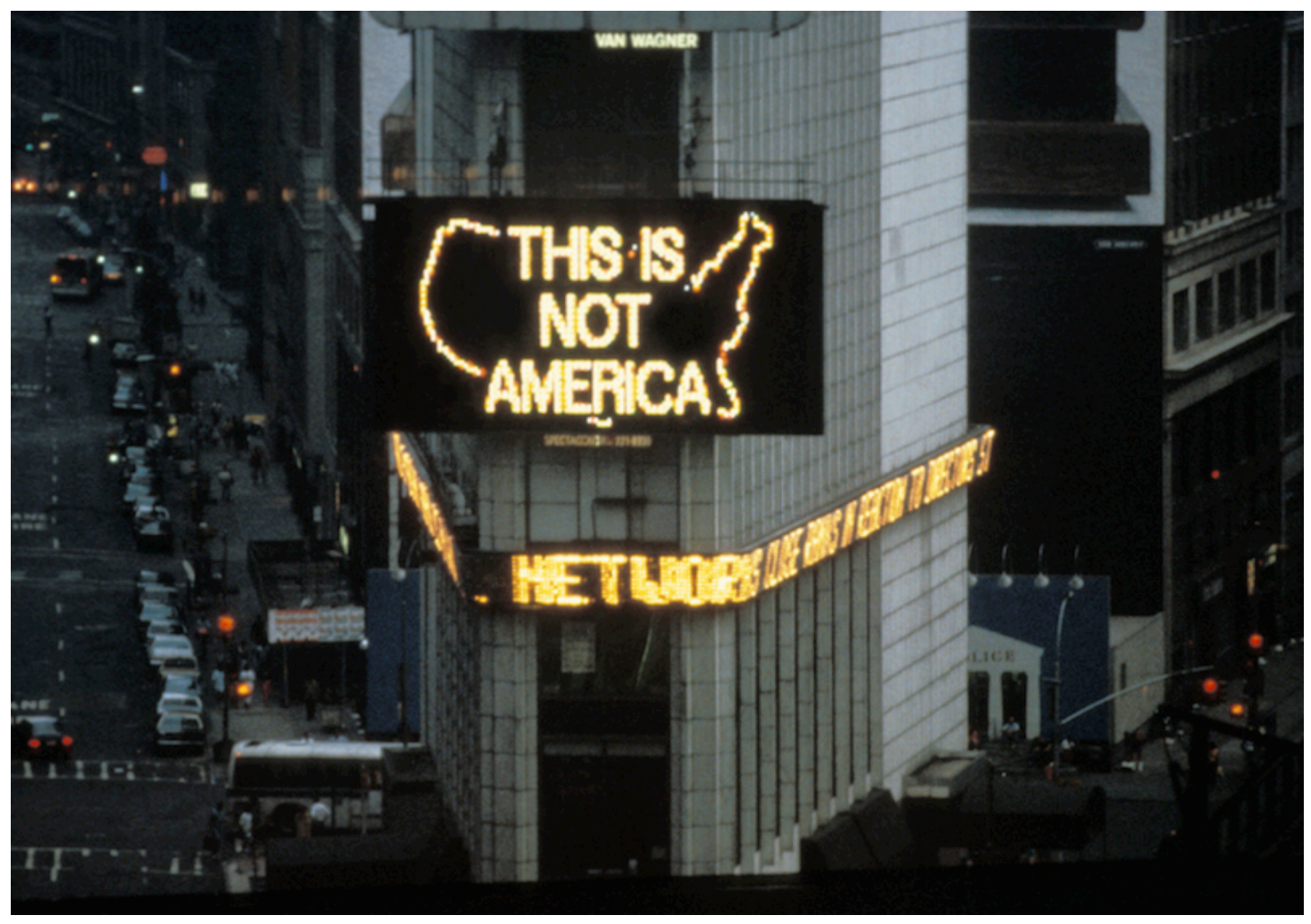

Figure 39. Alfredo Jaar, A Logo for America (1987), commissioned by the Public Art Fund for the Spectacolor Sign, Times Square, New York, April 1987. Photo courtesy of the artist and Galerie Lelong, New York (C) Alfredo Jaar.

Toronto's artistic and curatorial collective Public Access provides a similar example. Their project Some Uncertain Signs (1986), sought “a new aesthetics of interruption” (Sloan $2006,224)$ by negotiating access to an electronic signboard on the city's main commercial thoroughfare in order to present the work of 22 artists, a group that included Wodiczko and Holzer. Public Access hoped to reach an expanded audience and to scramble the semiotic identity of the city. Theirs was a tactic of disruption and confusion in order to shake people and the city out of its spectacular slumber. Whether or not they achieved this is difficult to gauge, and this is true of public artwork in general, due to the diversity of the generally non-art public that passed by the sign and the difficulty of identifying and 
following up with spectators. What is clear is that the work of Public Access did not go unnoticed: one of the works, a piece entitled "Lesbians Fly Air Canada" by Lynne Fernie, had to be altered to avoid litigation from the airline. Air Canada clearly believed that Public Access would confuse audiences, as intended.

The work of Public Access and of the Artangel Trust and Public Art Fund can be seen as part of an important "process of contradiction" (226), a polemical and proactive gesture, that seeks to be replicated in other cities that share similarities in the standardized technologies of public display. As Connolly (2013) argues in her article on the changing "mediascape" of public art, public spaces have been altered significantly by the imbrication of media such that they require artistic interventions to once again be "temporarily imagined as public" (215), something that Public Access was able to do for a limited time.

The practices of curatorial groups like Public Access also highlight the need for art to reach beyond the primacy of museums and commercial galleries. Similarly, as McQuire (2008) argues, "A crucial role for new media art in public space is the potential to avoid the filter of sites such as the art gallery, and thereby engage audiences who might never cross that threshold" (149). Public art must meet a wider audience in unexpected public places and do so in ways that best suit this public stage-to raise the stakes of art by making it more central to "the production of social relations and forms of life" (Hardt 2009, 26), or to expose the relational role the public plays in this. The work of Lozano-Hemmer is particularly instructive of how art in public space can construct and reveal relationships between participants, viewers, buildings, images, and architecture in the way it turns buildings into interfaces and public spaces into interaction fields. His work, Body Movies 
(2001), a computerized projection-based shadow play and image-matching installation that has been presented at various times on architectural façades in public squares around the world, fosters boisterous behaviour amongst participants and interactions between diverse groups ranging from polite cooperation to playful interference. Josephine Starrs and Leon Cmielewski's sms_origins (2009-10) (see Figure 40) provides another example of an artwork naturally suited for public space rather than the gallery. In Melbourne's Federation Square, public screens visualized real-time site-specific statistics of migration patterns sourced from the text messages of participants. The installation mapped these crowd-sourced trajectories live on the screen, reorganizing electronic telecommunication and elements of personal narrative through a tangibly collective and civic experience.

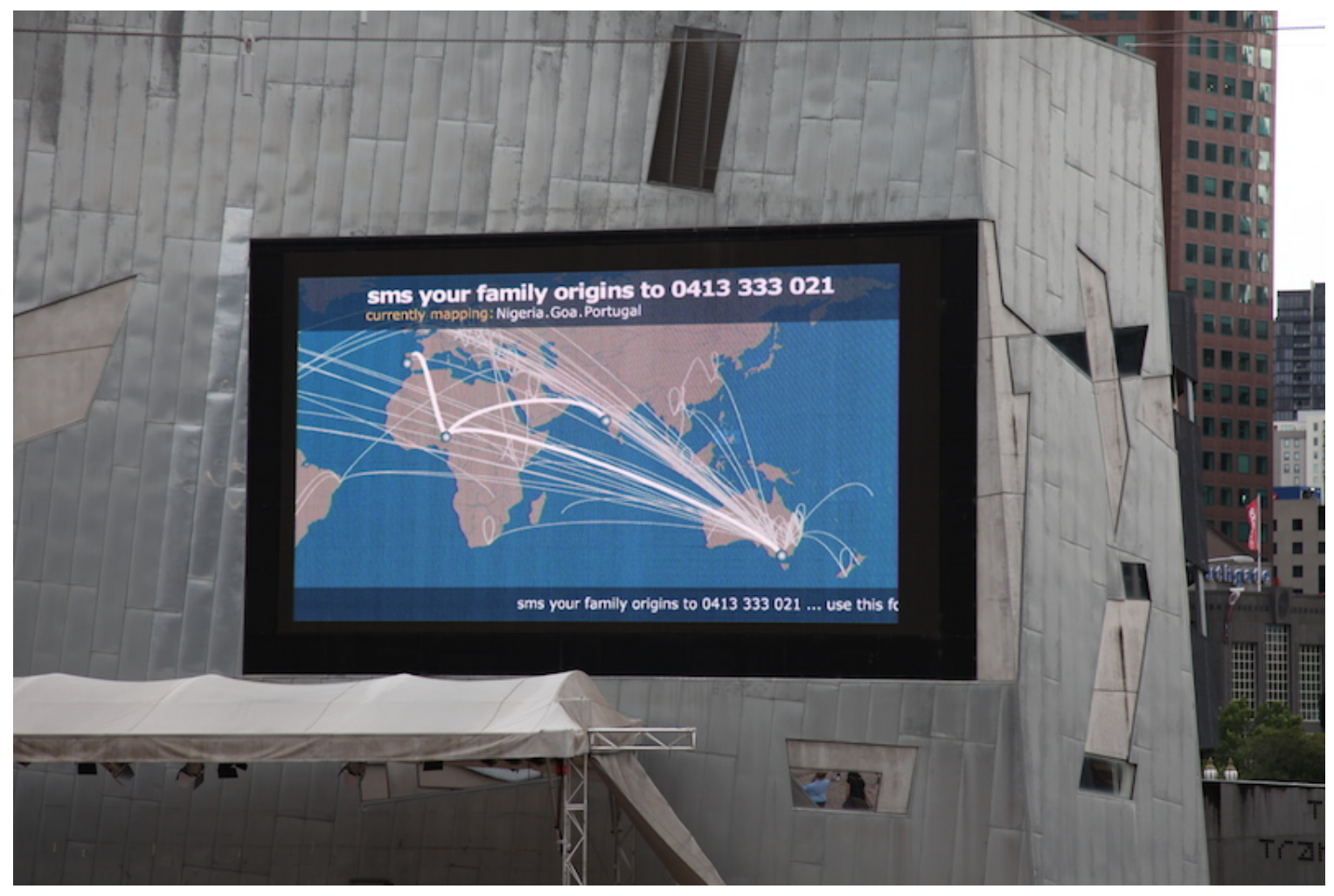

Figure 40. starrs \& cmielewski, sms_origins (2009-2010), Federation Square, Melbourne. Photo: Josephine Starrs and Leon Cmielewski, courtesy of the artists. 


\section{Massive Media}

While advancements in technology have opened up new sets of possibilities for artistic expression and experimentation in the public realm creating an expanded field that includes on- and offline forums for participation, feedback, and control, the public art of massive media-that is, emerging practices and places of exhibition that combine expressive architectural-scale elements (in the form of urban screens, public projections, or media façades) and telecommunication elements unique in their physical scale and geographical reach-requires new curatorial strategies and theoretical frameworks to understand and support their potential for creativity and criticality in the city.

Two concepts emerge that are particularly useful for understanding the relationship between the combination of media fragments and the observer in contemporary massive media scenarios. These are Murphie's (2004) concept of "transversal” subjectivity and Verhoeff's (2012) concept of the "composite dispositif." When interpreting the term dispositif, Verhoeff draws from both Foucault's notion of social control (1980), and Baudry's (1975) sense of subjectivity. Specifically, Foucault refers to the historically specific mixture of material and discursive practices that combine to contribute to some form of social control (i.e., a prison). Baudry, on the other hand, uses the term for the conceptual arrangement that, following from Althusser, interpellates the viewer into a certain subjectivity or point of view as a result of a coordinated technical apparatus (i.e., the cinema). In cinema, this apparatus is the equipment, such as cameras, film, the theatre space and other means that produce various effects, namely the capturing and maintenance of the viewer's attention on the diegesis. For massive media, this apparatus includes 
elements of cinema, architecture, urban space, mobile technologies and telecommunication.

As demonstrated in the preceding chapters, instead of a single dispositif, massive media create overlapping dispositives, given that their technical assemblage of urban media environments must be construed as a relational and contingent composite. Such a technosocial situation produces what Murphie (2004) designates as "transversal" subjectivities existing in many localities, or trans-locality. To understand identity as transversal within a composite dispositif is to understand it not as transcendent or fragmented, but as deeply enmeshed with other identities, media situations, and locations. This usage of the term "transversal" builds upon the term as defined in Guattari's The Three Ecologies (2000), in which he argues for the need to learn and think "transversally" in a globalized world enmeshed with technology, that is, in a way that considers the "interactions between ecosystems, the mechanosphere [technology], and the social and individual universes of reference" (43). The enacting of different profiles on multiple websites for various purposes is one phenomenon that demonstrates the way identity, for example, is expressed and performed transversally today: it is a social and individual universe of reference supported by a substantiation of a "mechanosphere." Trans-local, transversal identity is produced in a unique way through urban screens that are similarly networked or participatory yet more strongly related to physical space, and thus to real-world systems (social, ecological, and technological). Because data and communication now inscribe urban spaces and link disparate real-world locations, interactive screen-works embedded in space have the 
potential to utilize as well as enhance transversal thinking and pathways for creative and critical projects.

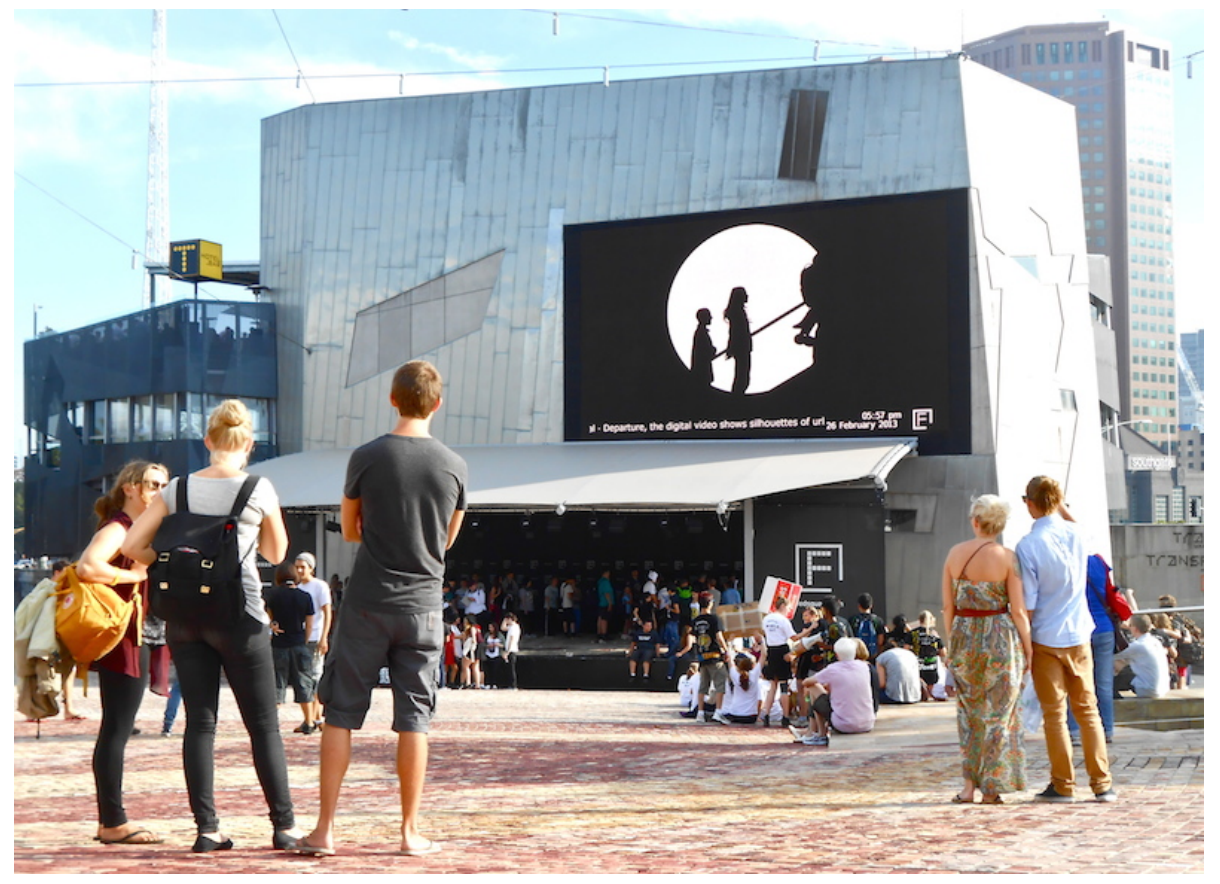

Figure 41. Tom Carr, Arrival - Departure (2013), Federation Square and Streaming Museum, Melbourne. Photo: Greta Jaeger, courtesy Art Plural Gallery.

\section{What People Have in (The) Common}

The question that must continue to be asked by artists and curators amidst the proliferation of massive media in cities is this: to what end? Will these sites simply deliver audiences more efficiently to the messages of advertisers, or can they endow city spaces with a greater potential for diversity in thought, aesthetics, and participation? Can they be used to generate important discussion about common issues or disrupt the status quo?

For philosopher Chantal Mouffe, public art should create spaces where shared concerns emerge, where dissent is promoted, and where the implementation of a radical, 
plural democracy is encouraged. In her view, instead of seeking horizons of intersubjective agreement, public art and monuments should establish spaces of common action rather than consensus. Her ideal public sphere, then, would be one embodied in artworks that further dissensus, that is, they refuse to simply commemorate or celebrate and instead foster common ground for debate $(2007,4)$. As Mouffe notes, "What is needed is widening the field of artistic intervention, by intervening directly in a multiplicity of social spaces in order to oppose the program of total social mobilization of capitalism" $(2007,1)$. Certainly, the early examples of public art interventions by groups like Public Access aimed and succeeded, to a degree, in doing just that-challenging the dominant consensus of urban spaces as places of uninterrupted commercials that leave little room for negotiation or heterogeneity.

What the transversal, composite dispostif of large-scale telecommunication networks ventilated through expressive architecture and public space can offer is both a potential site of contestation and a site where shared concerns might emerge from a new conception of the public. Can we communicate with and through public space? How? With what? What can and cannot be said? Who can speak? These questions evoke a new conception of what we might call "the common," or a material and social grounds for the necessity of politics. "The common," in this sense, as Michael Hardt (2009) argues, emerges as a dominant economic and social mode of public space. These questions also evoke what Rancière (2000) calls "the distribution of the sensible," or the way that our surroundings disclose themselves and our positions within them to us. As demonstrated in the examples of the preceding chapters, digital networked urban screens and public projections certainly 
change the parameters and the stakes for public space and public personhood in these ways.

A public art of massive media might be seen to harbour a responsibility to coordinate a relationship between contemporary technology, politics, and economics, constituting a commons "oriented towards the production of social relations and forms of life" (Hardt 2009, 26) that oppose those already given by corporate, commercial, technical, and civic agglomerations and that harness the specific advancements of coordinated technologies that we find in public space. The responsibility for "giving form and meaning to degrees of freedom opened by the progress of technology," as Paola Antonelli $(2008,9)$ notes, following closely from McLuhan, can be seen to fall to artists, designers, theorists, and curators of new media in the context of the public sphere. An important element of this technology for Antonelli is "elasticity"-seamless movements between scales and contexts of space and information $(2008,4)$. The digitally networked screen, linked to a mobile phone for example, as in the aforementioned sms_origins, mixes the public scale and context of the city and its messages with the private scale and context of the mobile phone as well as the private world of information that is ported through and stored within it. The attention switching that must occur to oscillate between these contexts is designed to be as seamless as possible, or coordinated such that an effective composite dispositif is created where multiple contexts of information (the city, the public screen, and the mobile screen) work together to recenter an audience, delivering them individually to a collective experience that identifies with no specific place or time yet, in a way, inhabits them all. As such, the dynamic characteristics of plurality, transversality, relationality, and networked 
connections engendered by the interfaces of massive media hold within them the potential for enacting new forms of the commons and being public.

An important function of supporting a practice of public art for massive media lies in curation, and particularly in an extension of the debates and methods developed in the curation of new media art. In Beryl Graham and Sarah Cook's analysis (2010), the curator of new media art must be a translator or interface between established structures and art forms and new, emerging forms and technologies in order to initiate important dialogues about space and materiality, ephemerality and permanence, and cybernetics and participation. One example that Graham and Cook provide regarding new media art curation is Learning to Love You More (2002-2009), a project that existed both as a website and a series of non-web presentations of work made by the general public in response to assignments posted by artists Miranda July and Harrell Fletcher on the project's website. Participants accepted assignments, such as “\#30 Take a Picture of Strangers Holding Hands" and “\#67 Repair Something," sending in documentation and reports as required (Fletcher and July 2002-2009). This work was then posted online under name(s) the participants chose for themselves. Here, the artists used digital networks to coordinate and encourage participation, sharing, amateur art making and temporary performances and interventions.

Along the same lines as Graham and Cook's work, Joasia Krysa (2006) adds the observation that the digital tools and techniques that are now incorporated into arts practices are also available and transformative to the curatorial process itself. Again, Learning to Love You More stands as an example as it taps into the Internet's ability to 
communicate to a transversal audience as well as its public archival function, essentially transforming the curatorial process through technology into the creation of digital interfaces and instructions that facilitate collection, contextualization, and presentation. As such, curatorial work after new media can also be understood to be composite and transversal, shaped by technological networks, elements of software, and their protocols. Together, what these theorists and curators point to is a changing field of curatorial and artistic practice by way of networked, digital media that affects all fields of artistic production and presentation.

Large-scale public networked urban experiences centred upon expressive architectural surfaces directly address and shape the urban experience in a cosmopolitan, globalized, post-digital world, opening up new ontological and epistemological possibilities for citizens, the city, and curatorial practice. The Berlin-based Connecting Cities Network and New York-based Streaming Museum are two recent examples of curatorial organizations primarily concerned with the challenges of massive media: curating within networks of media, urban sites, and trans-local audiences, and developing an emerging commons through massive media forms. In the following section I describe the history, mandate, technical tools, administrative structures, and recent projects of each organization, interspersed with perspectives gleaned from personal conversations and collected interviews of key figures, initiator and artistic director of Connecting Cities, Susa Pop, and founder and creative director of Streaming Museum, Nina Colosi. These organizations were chosen as they both aim to operate on a global scale, have an established track-record of work in the field that spans at least the last ten years, and have 
strong visions for what the future of large-scale, networked, public interactivity through urban media environments and public art should be.

\section{Connecting Cities}

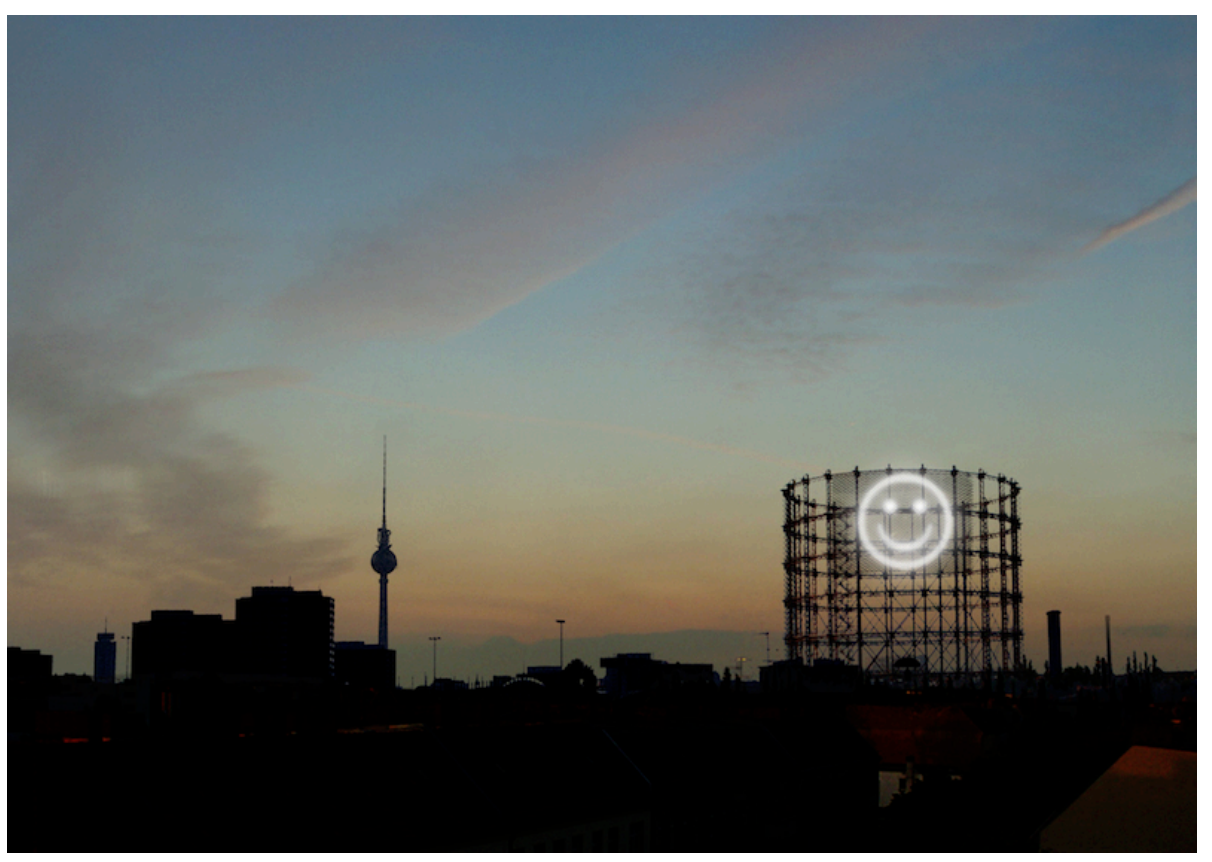

Figure 42. Richard Wilhelmer, Julius von Bismarck, Benjamin Maus, Mood Gasometer (2008), Media Façades Festival 2008, Berlin Gasometer, Berlin. Photo: courtesy of the artists. 


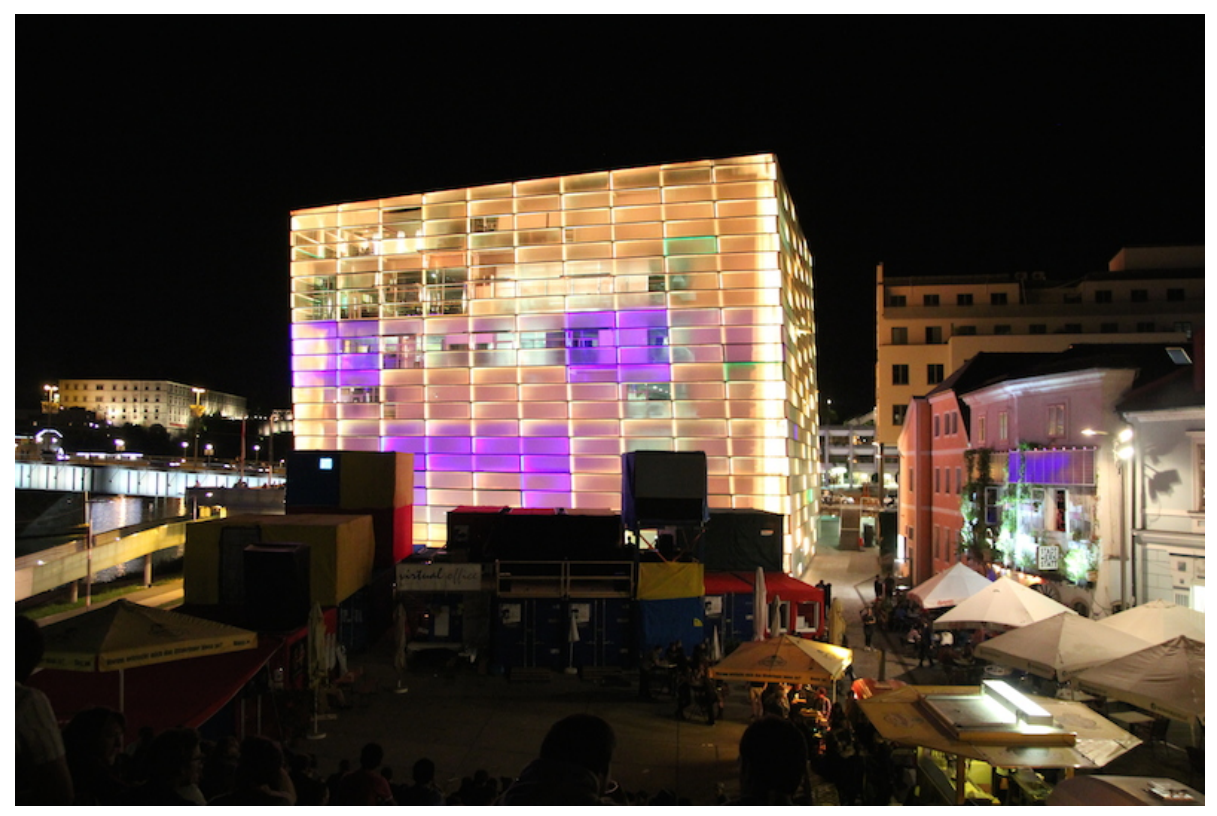

Figure 43. h.o, Connecting Monsters (2013), Connecting Cities, Ars Electronica Center, Linz, Austria. Photo: Pascal Maresch, courtesy Ars Electronica.

Berlin-based Connecting Cities seeks to coordinate the commissioning and circulation of artistic and social content across a primarily European (but increasingly worldwide) network of media façades, urban screens, and projection sites. They can be seen to be developing a networked, public art "commons" (Hardt 2009) that incorporates transversal publics and habituates audiences to new engagements with and across connected public spaces. They also help to develop infrastructure and expertise to foster experimentation and artistic freedom in these emerging techno-social scenarios (Connecting Cities 2014a) that increasingly depend on the creative use of the complex composite dispositif of public space, various sensors and screens, and networked participation.

Supported by the Culture Programme 2007-2013 of the European Union, Connecting Cities organizes and coordinates a network of curators that currently includes 
partners from various arts and cultural organizations on five continents. It has grown out of a number of curatorial initiatives that began with the formation of Public Art Lab (PAL) in 2003 by curators and cultural producers Susa Pop and Mirjam Struppek. Since 2007, PAL has focused on media-based art in the urban context that explores the potential for urban screens and media façades to act as communicative beacons and transfer points between citizens and cities (Connecting Cities 2014a).

Initial forays into this field by PAL were met with difficulties associated with working with existing corporately owned infrastructure. Two screens that PAL experimented with were the Nightscreen-Gasometer and O2 World arena façade in Berlin. With both screens, one located in an area of accelerating gentrification, the other functioning primarily as a sign for a new science and research centre, initiator and artistic director Susa Pop found that it was difficult to communicate with the corporate entities that controlled the screens and that artistic messages were often lost amidst regularly scheduled, non-artistic programming that was geared towards short impressions for passersby on foot or in vehicles (May 22, 2014, phone call with author). Instead of grappling with the productive tensions inherent in presenting art between commercials, Pop and Connecting Cities' focus was to "reclaim the screens" in order to explore their "socio-cultural potential" and "open them as community platforms and digital stages to connect cities and citizens with artistic scenarios" (Pop quoted in Toft 2013b). This was something that was not seen to be possible when only a mere sliver of the total screen time was available to engage audiences. Unlike earlier public art organizations interested in public screen infrastructure such as Toronto's Public Access, they were not seeking to 
create an "aesthetic of interruption" (Sloan 2006). Instead, their focus shifted to continual experimentation in creating a new public sphere supported by a more sustainable commons centred upon urban screens and multiple, focused encounters with a developing audience.

According to Pop, it was in 2010 that attention shifted to the idea of "connecting" cities through large public networked displays to explore new artistic scenarios that were inclusive of local culture but also engaged in a "trans-local" dialogue (Pop quoted in Toft 2013b). This was further explored through the Media Façades Festival Europe 2010, which connected seven European cities via urban screens and media façades and joint broadcasting events, essentially engaging a new transversal real-time common that was just then becoming a technological possibility. By 2010, it had become apparent that urban screens and media façades were operating through internet technologies and thus offering new possibilities for connections that were both networked and physical.

The current mandate of Connecting Cities covers several basic tenets that emerge from these early debates and technological shifts. For example, Connecting Cities opposes the commercial use of urban media. Instead of advertising and branding, Connecting Cities is concerned with linking citizens within and across cities. Their networked displays "provide space for intercultural encounters and exchange" (Connecting Cities 2013a) and ask questions pertaining to the nature and possibility of transversality in contemporary cosmopolitan culture:

Can urban media façades become a catalyst for shared encounters in an identity-creating temporary field of interaction across the border of time and distance? How can we use art projects to connect local public virtually with 
other places? and; What are the expectations and visions of our neighbours? (Connecting Cities 2013a)

Connecting Cities is currently focused on imagining and creating new democratic forms from the elements of technologized urban space, supporting temporary trans-local public spheres that seek to furnish conversations about potentially divergent "expectations," following somewhat from Mouffe's understanding of the public sphere as a site for contestation.

Furthermore, Pop has stated that the goals of Connecting Cities are to "create in people's mind not only awareness but an understanding of the current evolution of our urban environment" that sees public space as "a space for creativity, visibility, and exchange of culture where a connected public sphere is created" (quoted in Toft 2013b). This goal clearly follows from a contemporary understanding of a public sphere that must address a "distribution of the sensible" Rancière (2000) through the lens of hybridity and relationality, reminding and educating various groups about where and how they might be able to engage in public discussions related to common concerns. Such theories and goals find a place in their latest round of programming (2013-2015) in which Connecting Cities explored the themes of the Networked City, focusing on city-to-city interventions, the Participatory City, focusing on engaging the spaces around the media façade both locally and trans-locally, and the Visible City, focusing on creating real-time windows into environmental and contextually relevant data. The mandate of the Connecting Cities Network has grown out of a perceived disconnection and distraction caused by conventional uses of urban media and is now directed towards the fostering of local and 
trans-local connections and understandings, particularly through experimental networked scenarios.

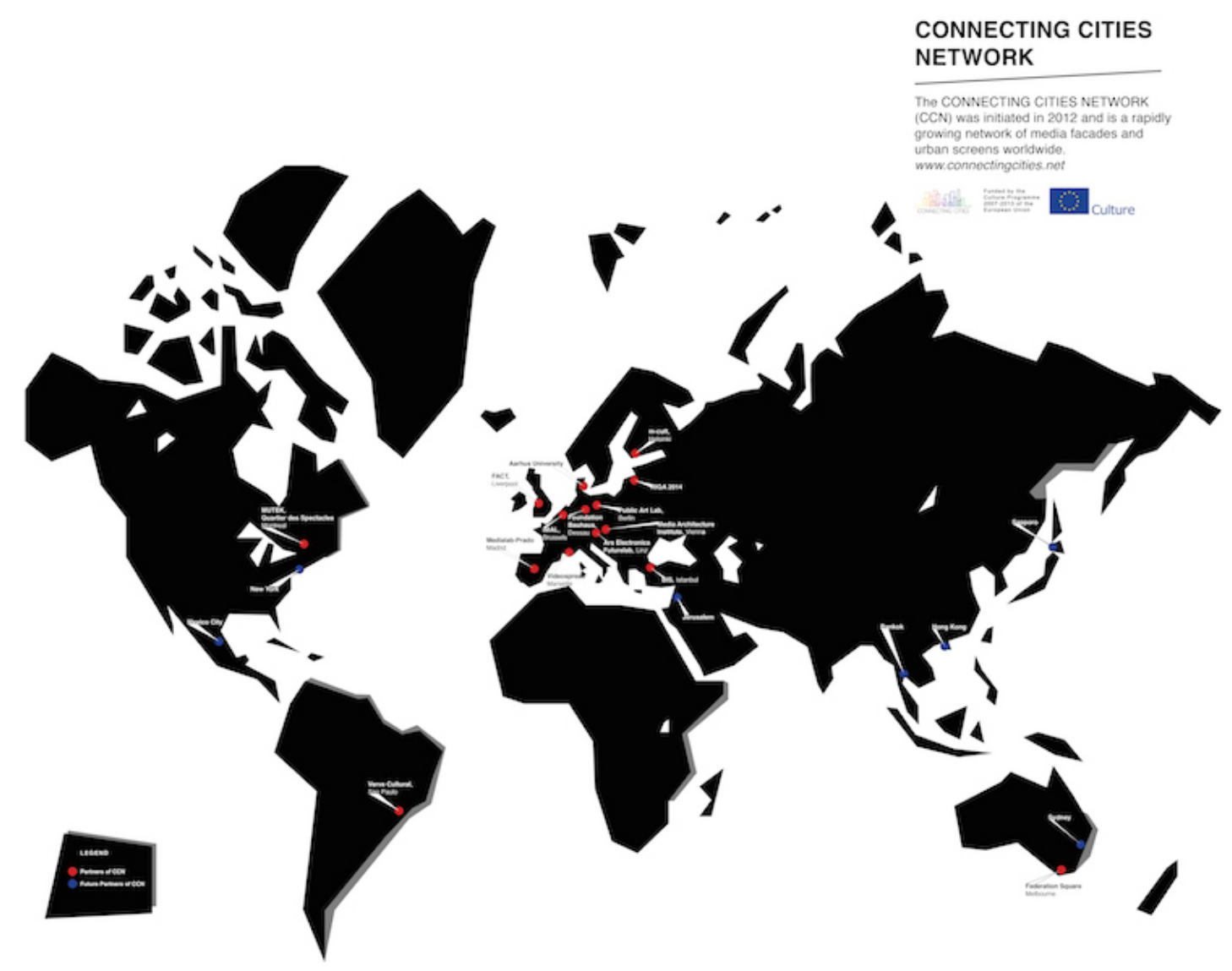

Figure 44. Connecting Cities Network. Courtesy Susa Pop.

Connecting Cities' focus on the trans-local and the network extends to the tactics of their curatorial approach that seeks to connect curatorial expertise and infrastructure, essentially creating a temporary curatorial commons and trans-local exhibition space for each project. This is vital for effectively addressing a newly envisioned hybrid and relational public sphere. Connecting Cities taps into local knowledge and avoids the constraints associated with commercial screen environments by building relationships with and 
between curators at various arts and cultural institutions that have their own media façades, such as Quartier des spectacles in Montreal, Medialab-Prado, Federation Square in Melbourne, and Bauhaus Dessau. Often these institutions have their own local mandates that must be coordinated with those of Connecting Cities. For example, Federation Square's "big screen" must share its screen time for commissioned artworks with live sporting events and promotional content for the site, whereas Medialab-Prado is concerned solely with the production, research, and dissemination of digital culture.

A recent project that reflects the coordinated efforts of the curatorial network of Connecting Cities and their mandate is Binoculars to... Binoculars from... (2013) by Varvara Guljajeva and Mar Canet Sola (see Figure 45). Part of the "Networked City 2013" program, Binoculars involved the installation of "binocular-like" kiosks in public squares around Europe that would provide a view of similarly equipped but otherwise unidentified remote locations. With coordination and support from Connecting Cities, the project was produced by FACT (Foundation for Art and Creative Technology) in Liverpool, and adapted by Ars Electronica in Linz, Medialab-Prado in Madrid; Public Art Lab and Foundation Bauhaus Dessau in Dessau, Public Art Lab and ikono in Berlin; iMAL (Centre for Digital Cultures and Technologies) in Brussels; m-cult in Helsinki; Federation Square in Melbourne; and Foundation Riga 2014 in Riga. After its completion, information about the project was included on the Connecting Cities "Technical Framework" website describing the hardware required for the façade, computers, lights, interfaces (a detailed technical diagram is available on the site), networks, and a list of technology required both at the production city and the presentation city. 


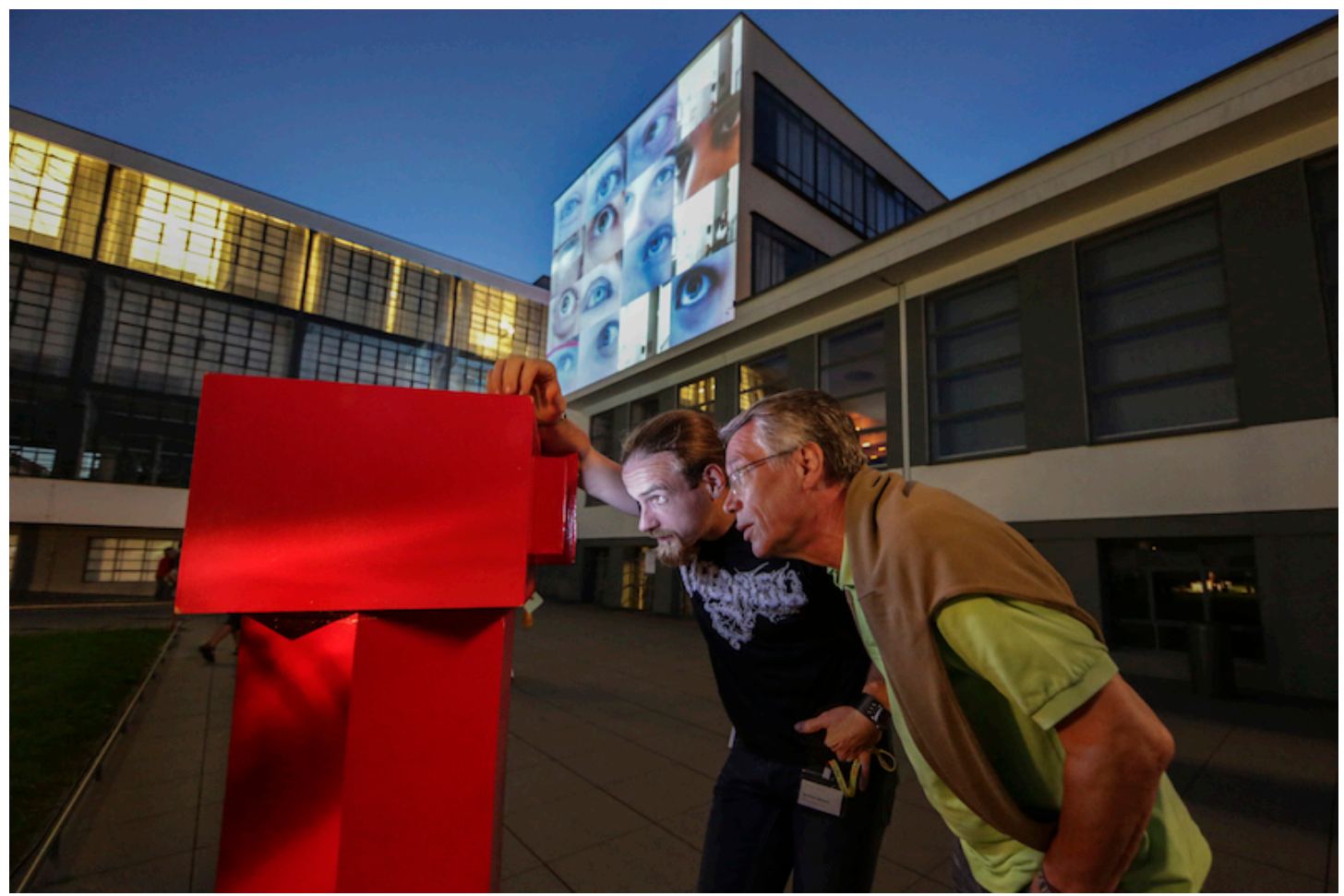

Figure 45. Varvara Guljajeva and Mar Canet Sola, Binoculars to... Binoculars from... (2013), Connecting Cities, Bauhaus Dessau. Photo: Ruthe Zuntz, courtesy Public Art Lab.

Binoculars was inspired by the panorama binoculars one might find at lookout points in various cities. While observing other locations from the binoculars, viewers eyes are captured and displayed on the urban screen of the observed place in real-time. The experimental proposition, facilitated by a combination of technologies centred upon large urban screens, is one of trans-cultural and trans-local connection that constitutes a new "gaze" oscillating between surveillance, tracking, play, and telecommunication: all elements that are increasingly part of a trans-local commons that constitutes economic and social transactions such as social networks and energy consumption. Binoculars shows how such a nexus of concerns can be made into a more public matter by engaging a composite dispostif of networks, screens, and public space. Via Binoculars, Connecting Cities 
demonstrates an emerging commons and the media and spaces we can use to engage and contest it, thus spawning questions and potential debates about the possibilities and perils of networked technologies, vision systems, global consciousness, and public displays.

As noted earlier, the coordination of display contexts necessary for this is achieved through a network model that harmonizes the curatorial goals of Connecting Cities with the local mandates of partner organizations. While Connecting Cities does observe and incorporate feedback from their ongoing presentations, missing from their curatorial process is the direct input of the public they seek to serve. Although this does enter into the curatorial process to varying degrees depending on the outreach efforts of partner organizations, it remains provisional and conspicuously absent from their mandate.

Officially, the curatorial process of Connecting Cities involves meetings, workshops and the provision of a centralized database of projects and protocols. Typically, a call for artists is sent out, outlining general guidelines and themes. Then, artists are pre-selected through a collaborative process involving the host organizations. A final list of artists is provided to curators in each participating city and they select at least one artist to be produced locally for the event as well as deciding on suitable sites for the work. Selected artists are invited to a workshop where, together with curators, they develop projects, concepts, and technological setup and circulation plans with the other participating cities, ensuring that they are in line with the different local circumstances and production conditions. The result of such a collaborative and networked process has been the establishment of an infrastructure of diverse urban screens and media façades in regards to resolution, size, daylight compatibility, content management systems, internet connectivity 
and site-specificity. This system also facilitates the leveraging of local and national governments for funding, creating the conditions for the sharing of curatorial expertise, and nurturing audiences for large-scale, networked public works. Such coordination and leveraging serves to highlight the difficulty of any kind of bottom-up, grassroots approach to controlling and directing the uses of large urban screens, something that has not changed significantly since early public screen experiments. This is a distinct weakness or limitation to constructing a commons from public screens.

While Connecting Cities remains somewhat deficient in finding ways to incorporate citizens into the curatorial process, they excel at using network tools to shape and support the curatorial process amongst fellow curators, infrastructure owners, and participating artists. A separate website entitled Connecting Cities "Technical Framework" (Connecting Cities 2013b) helps to provide continuity, ease, and cohesion between the disparate technologies and contexts of the curatorial network. The "Technical Framework" site is both a repository and archive of previous works, as well as a site that collects information about the specific technical capabilities and constraints of each façade in the curatorial network. Links to key technologies commonly required in media façade installations such as Processing, Raspberry PI, and OSC are complemented by a link to the Connecting Cities GitHub community that serves as a forum for troubleshooting problems specific to these sites and technologies.

In this way, the work of Connecting Cities certainly reflects an ongoing shift in curatorial attention from objects to processes and dynamic network systems. Media theorist Sean Cubitt suggests that a measure of success for any work in a digital milieu depends on 
a work's "breadth and depth and complexity of networks engaged and engendered" (2007, 313)-essentially its "elasticity" (Antonelli 2008, 4). Connecting Cities provides the means for breadth and depth of connection across networks of telecommunication and of global urbanity, and an elasticity of scales and contexts, something that is demonstrated in a project like Binoculars. Such a metric of success applies just as much to curating, particularly in scenarios as geographically dispersed and technically complex as Connecting Cities. Specifically, the Connecting Cities "Technical Framework" represents precisely what Krysa and Graham and Cook envision as part of the expanded field of curation for new media and networked systems; in short, it is a tangible instantiation of new possibilities for the organization of the curatorial process that includes software, protocols, and networks. At the same time, it refers back to very real, very material sites and social processes where the curatorial meets the concrete experience of exhibition. The Connecting Cities Network extends our idea of what curation is and reminds us that curating today cannot be separated from social and technological developments (Krysa 2006, 7).

Furthermore, Connecting Cities harnesses the potential of transverse publics and networked scenarios by situating themselves, and the works they select, as "interface actors" (Susa Pop, May 22, 2014, phone call with author)-following on from Graham and Cook's (2010) definition of curating after new media-orchestrating the performance and practice of public sociality across diverse contexts. This often involves finding ways to include the audience and habituate them to new rituals of public art practice and reception through the design of participatory protocols and cues. Pop cites Medialab-Prado's work \#ProgramaLaPlaza as a prime example of this (see Figure 46 and Figure 47). The project 
allows participants in any location (with an internet connection) to write code for Madrid's public media façade which can then be viewed on the façade immediately thereafter. Medialab-Prado then selects the best of the contributions to be shown as part of a one-week exhibition. The aforementioned Binoculars also works to propose a new mode of engagement, one that acknowledges a mutual desire to look and be looked upon between and amongst urban populations. Both projects seek to develop communities, audiences, and publics that leverage media façades through the local and trans-local interactions. The networked frameworks within which projects like Binoculars and \#ProgrammaLaPlaza emerge reflect an emphasis on greater circulation of these works and an open research component which helps the Connecting Cities Network develop as a form of DIT (do it together) activism (McKim 2014, 6) for public space and massive media, and solidifies their role as "interface actors" in community building. 


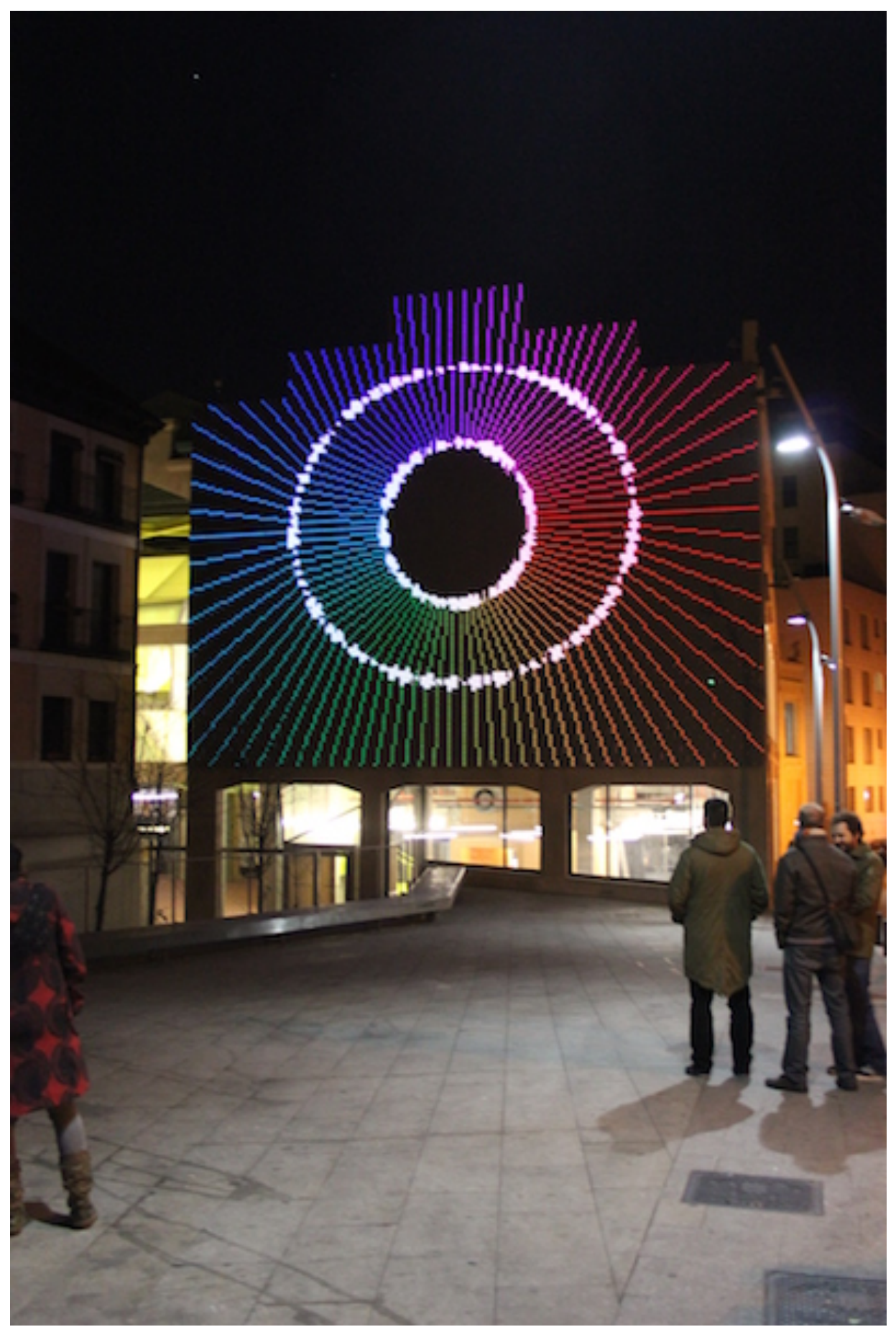

Figure 46. Frederic Granon, BigBro (2013), \#ProgramaLaPlaza, Medialab-Prado, Madrid. Photo: courtesy Medialab-Prado. 


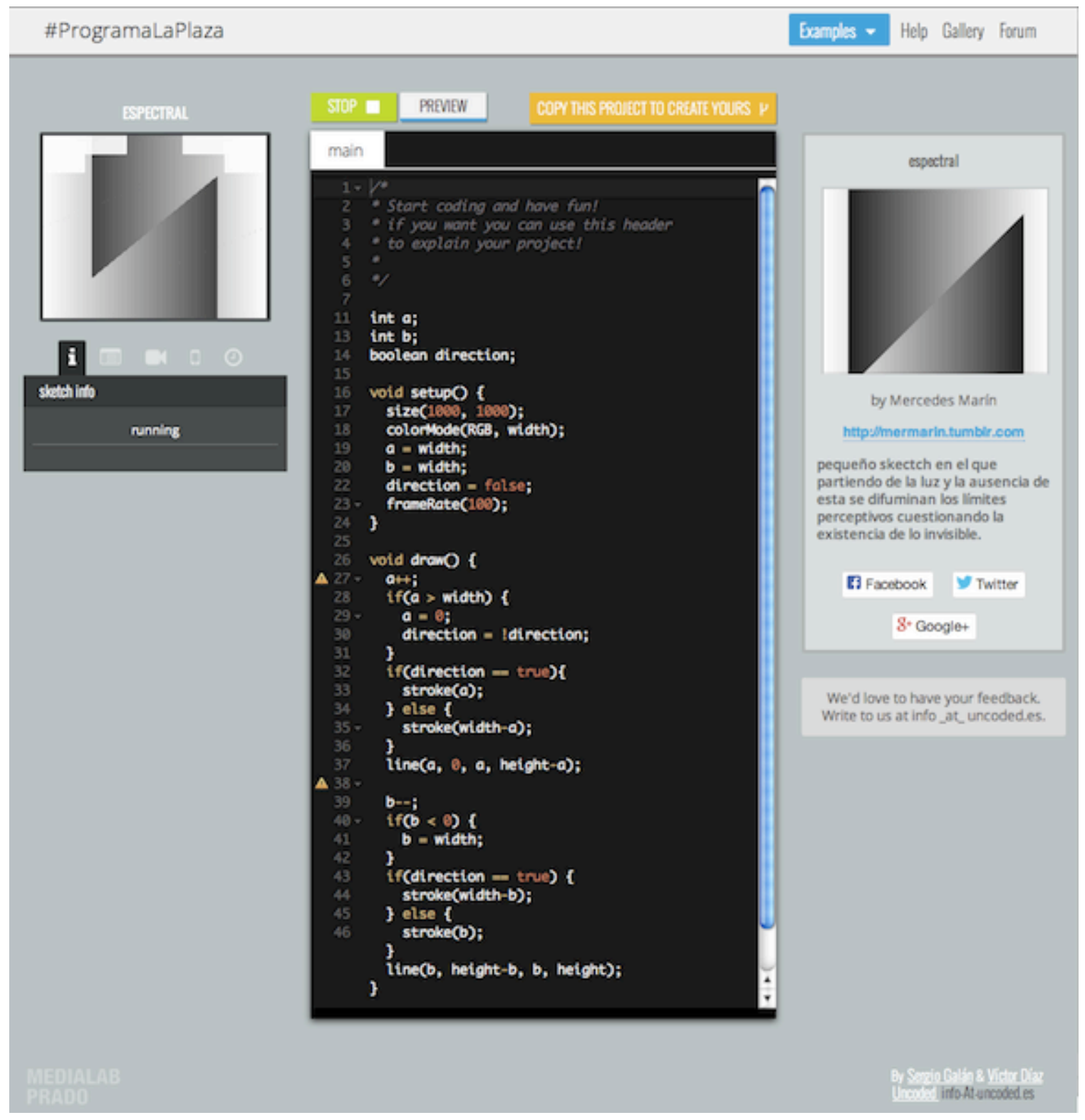

Figure 47. Medialab-Prado, \#ProgramaLaPlaza, screenshot.

Connecting Cities also demonstrates a refocusing of work for large urban screens that seeks to create and foster a commons or the foundations of a public sphere that address contemporary ontological and epistemological issues: what or where exactly is the space of contestation that we share as urban, networked beings? Under what terms do we encounter and address one another? And to what degree can we see ourselves reflected 
back to ourselves in our trans-local spaces? As noted earlier, we can often only see ourselves reflected back to ourselves and address one another in meaningful ways in public space through the affordances opened up by artists concerned with these questions and the curatorial network that supports them. In this sense, both the artist and curator harbour a responsibility to create rich spaces of interaction and contestation that blend networked and trans-local participation. They also harbour a responsibility for understanding that the commons exists in a hybrid, contingent space that combines code with bricks and mortar, the screen with the street. So, while the symbolic spaces of large public display are important focal points in these works, it is the amorphous spaces of interaction and social contexts and forums in the case of Connecting Cities, such as the "Technical Framework" enabled through various works, that support enriching, participatory hybrid environments.

That said, the spaces created and engaged by these projects cannot be seen as going as far as becoming sites of dissent or radical, plural democracy, as is hoped of critical public art by Mouffe. Particularly in Binoculars, there is a reduction of participation and expression to the eye that is captured, transmitted, and recombined across public spaces. In many ways, the suppression of speech or text is a necessity of trans-local dialogue, namely that it must sidestep the complexities and specificities afforded by language in order to engage audiences that do not necessarily share a common language. The connection, then, is an emotional and symbolic one. While this may lay the groundwork for future dialogue, and certainly was a catalyst for local dialogue amongst participants and viewers at the various locations of the installation, the result is a minimal quality of debate with respect to the formation of a democratic trans-local public sphere. Despite this, Binoculars is not 
merely a spectacular aestheticization of the city or a facile entertainment, as McKim warns (2014). Rather, it presents new possibilities for urban screens and an embodied transversality amongst diverse locations that can help to build temporary but powerful international connections amongst city dwellers, especially as audiences and their attendant interfaces for communication become more sophisticated. This addresses the ever-present challenge of developing an audience that is willing to be patient enough and skilled enough to participate with remote contexts across public spaces. For example, the elasticity in space and time created by the composite dispostif and transversal identities of Binoculars might be read in relation to the recent rise in popularity of Meerkat and Periscope (Tweedie 2015), two applications that allow individual users to broadcast live feeds from their smartphones. This phenomenon has even been taken up by news organizations such as The Guardian (Lewis, Swayne, and Jacobs 2015) that have used it to report on breaking news stories and events such as the civil unrest in Baltimore in the spring of 2015. This may be something that in conjunction with massive media could be directed to confront international issues such as environmental degradation and human rights through the ongoing habituation towards seeing through another's eyes.

At this point, it is important to consider the relationship that massive media works have to the concept of the spectacle. While large-scale works may be enticing and distracting due to their size and visibility, a condition of Guy Debord's (1995) definition of the spectacle, they are also activated by participation through which criticality and variation can be performed. Massive media spectacles thus contain a critical element through their openness to participation. That said, participation and the novelty of trans-local 
experiences are a double-edged sword: while they can disrupt the spectacle of commodified public spaces, they can also function more complicity to deliver audiences to corporate and commercial goals of promoting telecom companies, creative cities, and entertainment districts. This is something of which curatorial groups must be cognizant.

As noted earlier, due to the constraints associated with commercial screens, Connecting Cities moved away from occupying the gaps between advertisements. While previous art organizations using public advertising boards, such as the Public Art Fund and Artangel, targeted commercial spaces in order to critique them on their own ground, and to insert art into a larger cultural economy (Connolly 2013, 206), Pop observes that there are limitations to what can be done within the commercial frame. Typically, no segments longer than a few minutes are available, as was the case with the Times Square Spectacolor sign, thus the critique of spectacle in this form was particularly limited. As Pop (2014) notes, "All that can be shown in that timeframe are videos or a trailer; you cannot really experiment with them." By partnering and developing relationships with sites that consistently host art and experimental programming such as Medialab-Prado, Melbourne's Federation Square, or Montreal's Quartier des spectacles, funded exclusively through government grants, civic partnerships, and funds from commercial tenants (in the case of Federation Square), Connecting Cities can instead pursue an agenda of cocreation/curation and "urban prototyping" where new public interfaces can be tested through prolonged iteration and observation (Susa Pop, May 22, 2014, phone call with author). It is also important to note that most of these sites in the Connecting Cities Network are designed with substantial embedded media infrastructure, including sensors, 
lighting, wiring and other permanent elements such as touch-screen kiosks that allow these spaces to function as user interfaces-an expanded relational commons. Infrastructure control, then, becomes one of the pre-requisites for Connecting Cities and the curation of massive media in general, and one of the key distinctions between curatorial aims that critique spectacle directly versus those that envision deeper encounters through large-scale public displays.

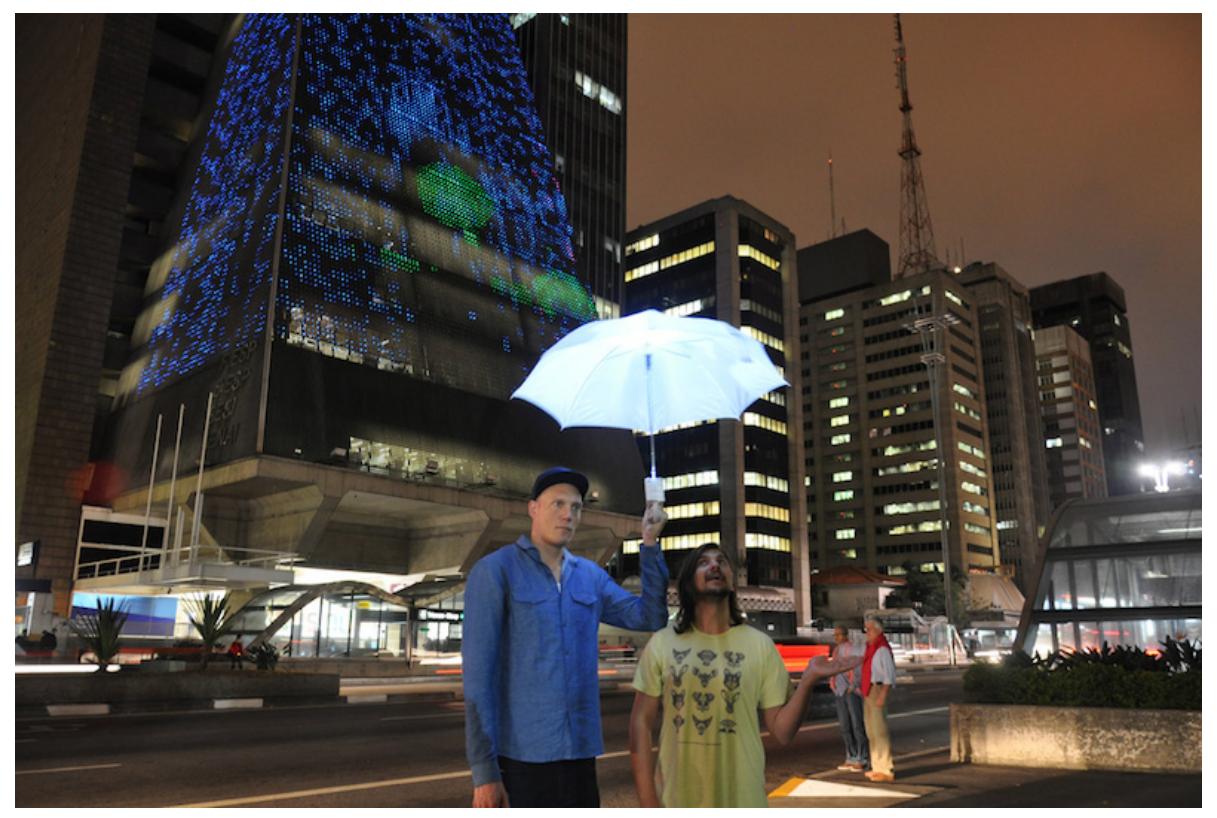

Figure 48. Pfadfinderei + The Constitute, Dancing in the Rain (2013), Connected Cultures Programme, Galeria de Arte Digital SESI-SP, Avenida Paulista, Sao Paulo, Brazil. Photo: verve cultural, courtesy Public Art Lab.

With an emphasis on experimentation, Connecting Cities has moved away from their original intent to "give these platforms back to people" (Susa Pop, May 22, 2014. Phone call with author), sensing that the highly provisional and restricted access to commercial screens and limited screen time did not lead to any lasting development of the 
medium and at worst furthered the detrimental impact of corporate interests in public space: interstitial art had become an ad for the ads and did little to foster new ways of being public. As mentioned earlier, Connolly (2013) argues that interventions are necessary when public space has become overrun by media messages. As the ecology of media changes, so too do the interventions that keep public space truly public, albeit temporarily (215). Instead of grappling with commercial sites that bury the art within the noise of a competing composite dispositif and programming, Connecting Cities has opted to intensify the already-built platforms that have existed explicitly for art audiences and community development from the start so that new publics can be imagined and tested with greater consistency and regularity.

\section{Streaming Museum}

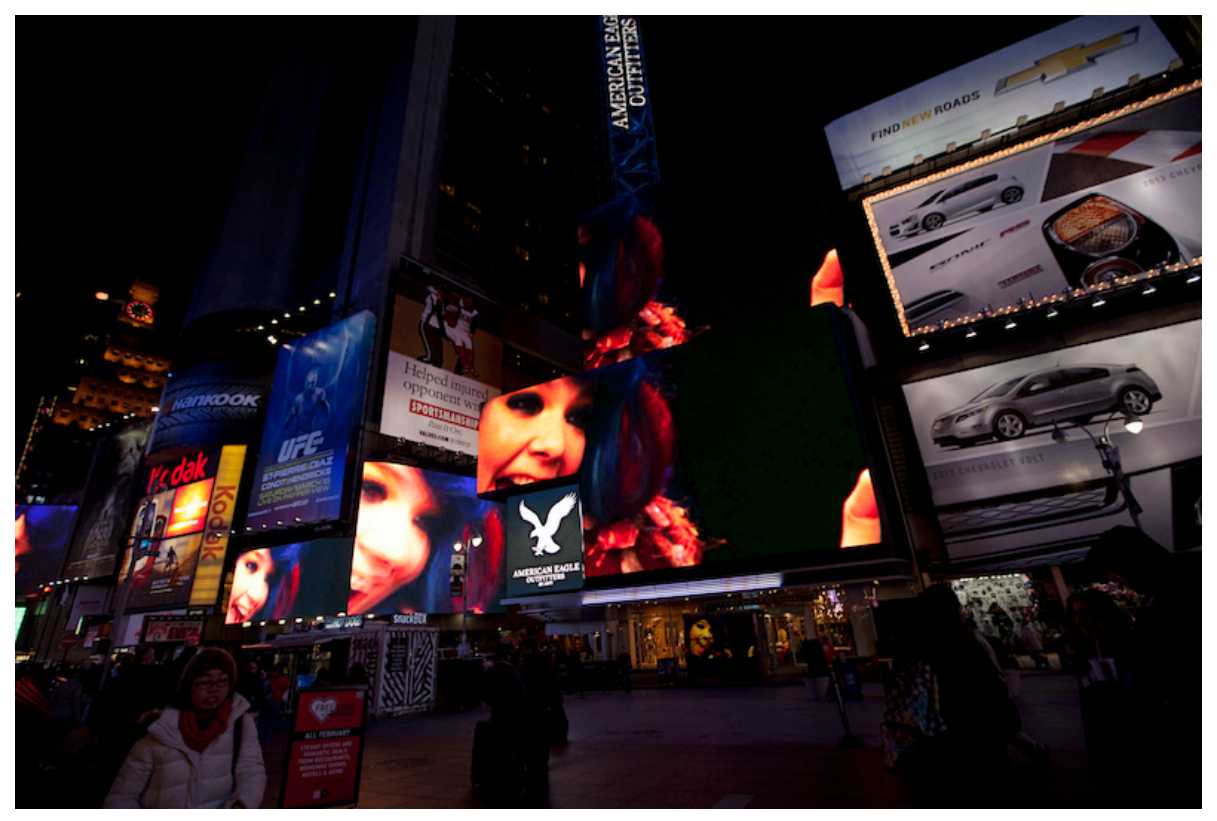

Figure 49. Björk, Mutual Core (2012), Times Square Midnight Moment, Streaming Museum \& MOCAtv, New York. Photo: Ka-Man Tse, courtesy Streaming Museum. 
Streaming Museum forms temporary partnerships with cultural and commercial centres to produce contemporary-themed art exhibitions on screens (including its website) and public spaces on seven continents (Streaming Museum 2013). Although based primarily in New York City (though they would contend that they are not necessarily located anywhere), the organization works with digital and physical infrastructure and curatorial networks around the world that suit a specific curatorial theme that governs a year-long cycle of programming. Some of their partners have included bitforms (NYC), Nam June Paik Art Center (Korea), and Zayed University (Dubai).

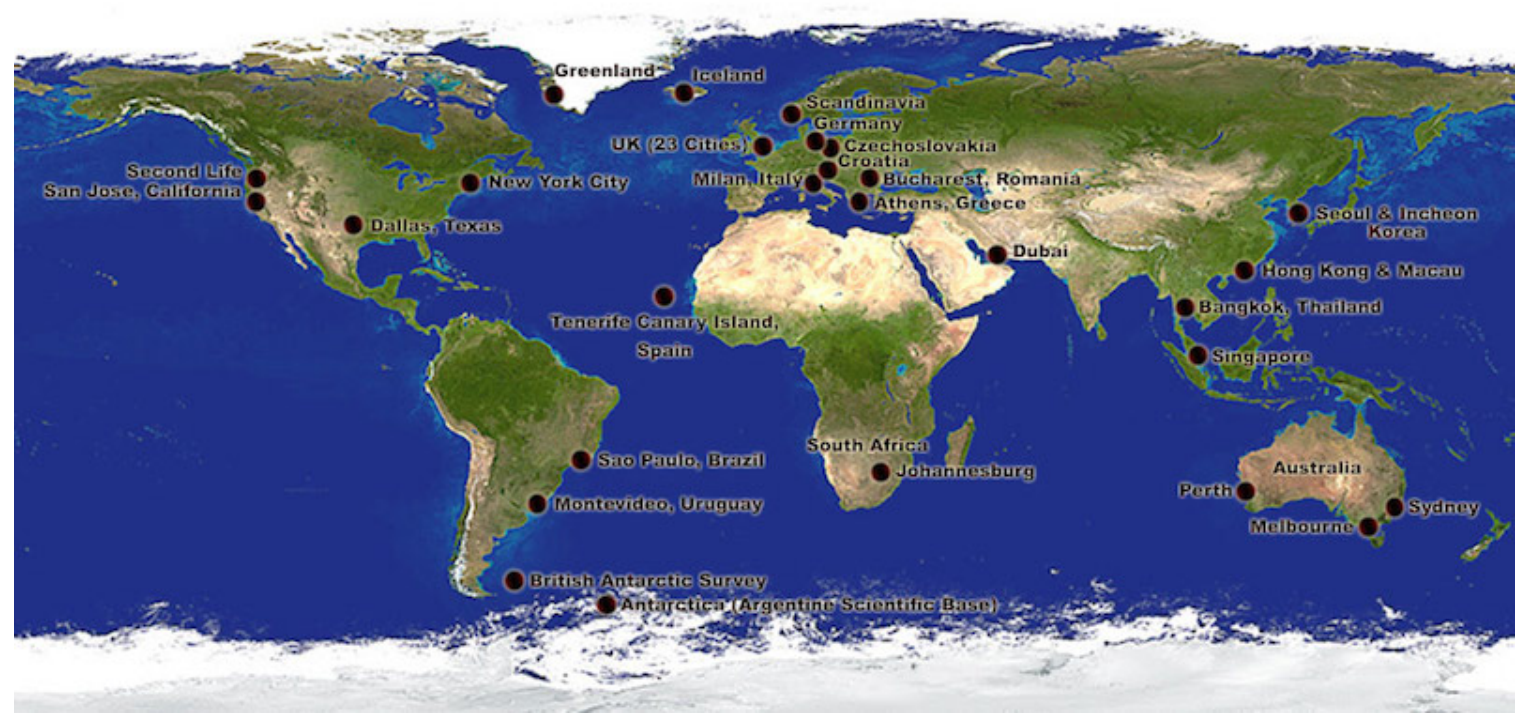

Figure 50. Streaming Museum Network, 2014. Courtesy Nina Colosi.

Streaming Museum officially opened on January 29, 2008, exhibiting Good Morning Mr. Orwell (1984), a work by Nam June Paik that was broadcast around the world in 1984. By coordinating public screens on every continent; Johannesburg, South Africa; Antarctica, Jubany Scientific Base of Argentina; Seoul, Korea; Melbourne, Australia; London, UK; 
Dallas, Texas; Montevideo, Uruguay; as well as Second Life, Streaming Museum was able to show Paik's work internationally once again. Just as Paik's work emerged at a time in which satellite television was seen as a viable site for the contestation of the tele-visual broadcast hierarchy that had been established in the twentieth century (Hansen 1993)-an exploration of the "positive and interactive uses of electronic media" (Paik 1984)-its appearance in the launch of Streaming Museum signified an equally optimistic view of the possibilities for art and cultural dialogue possible within the largely commodified confluence of large digital displays, digital mobile devices, and the Internet. Good Morning Mr. Orwell also represented a mixture of art and popular culture, appropriating a variety show structure but injecting it with performances by avant-garde artists such as Merce Cunningham, John Cage, and Joseph Beuys that probed the nature of the human condition in this new era of technology. As Nina Colosi (quoted in Toft 2013a), founder and creative director of Streaming Museum, noted, Streaming Museum "was launched at the cusp of the global expansion of the Internet and screen culture, including mobile, computer and urban screens." It sought to represent and explore the new ontological and epistemological possibilities of urban, digital networks established by the composite dispositif of interoperable media devices, transversal identities, and hybrid spaces.

Streaming Museum takes its name from the idea that the future of the museum is one that they believe should mimic the "streaming" forms of data that comprise cultural production today. A "streaming" museum eschews the solidity of built forms, opting instead for temporary instantiations on networked screens, while reaching its public either online or in hybrid public spaces supported by massive media. As Colosi (April 7, 2014, e- 
mail to the author) notes, it "goes where the people are." In this case, "the people" are assumed to be always in, on, or around screens. Exhibitions have traveled to over 65 locations on seven continents and StreamingMuseum.org since 2008. Streaming Museum adopts a touring exhibition model punctuated by coordinated launch events such as Good Morning Mr. Orwell. It thereby aims to cultivate a cosmopolitan pubic sphere that enables a global audience to address issues of media access, representation, and other pressing concerns such as sustainability and renewable energy that extend beyond national borders. Colosi says that the development of streaming media channels and practices on- and offline are the forerunners for an engagement in what she calls "a system of global governance," a kind of distributed United Nations supported by interconnected media and media spaces:

It is conceivable that increasing digital interconnection will accelerate the emergence of cosmopolitanism resulting in a league of "global digital cities" that could share in a system of global governance. This could enable the monitoring and distribution of renewable energies and education, health and other social needs to those areas of the world where it is needed. $(2012,52)$

Colosi crucially extends the optimism that informed Paik's early experiments with satellite broadcast to the level of the global digital city thus identifying the physical spaces (and challenges such as energy and health) as sites and subjects of concern that are enmeshed with technology and well suited to massive media scenarios that foreground transversality. Concurrent with Streaming Museum's ideals as stated by Colosi is a dedication to contributing to an "inspiration-and-information-with-social-value economy" (Streaming Museum 2013), that is, a sense that Streaming Museum should work with, not against or in explicit opposition to, the flow of capital and thus within the scenarios and networks of 
commercially owned urban screens. Thus, Streaming Museum's focus on "value" is reflected in their willingness to collaborate with corporate entities such as the Times Square Alliance in order to achieve maximum visibility, sustainability (by piggybacking on a profit-driven model where infrastructure is sustained and updated through advertising dollars), and exposure.

The technical and organizational structure of Streaming Museum reflects a shift to networked systems and experiences, as opposed to specified locations or institutions. As Colosi explains: "Streaming Museum is a new museum concept built with technological elements; it's not fixed in one place" (Colosi 2014). Thus, instead of approximating a curatorial network, Streaming Museum can be seen as curatorial technique that treats networks, both physical and digital (combined in the form of networked urban screens), as the "museum."

Streaming Museum consists of three permanent members, its founder and creative director, Nina Colosi, associate curator, Tanya Toft, and videographer, David Bates, Jr. with a number of curators and directors participating on an ad hoc basis. Essentially, Colosi begins with a curatorial theme, either one that she is interested in or relates to a particular project or festival they are working with. This is followed by a period of intense research and discussion with curators and artists (Colosi 2014). For example, Streaming Museum completed its Nordic Outbreak program in 2013, an exhibition of over 30 moving image artworks by Nordic artists curated for public space, which included partnerships with curators and organizations across the Nordic countries (such as a temporary screen at KIASMA in Helsinki), as well as with established partners around the world (such as Times 
Square Arts in New York City). In this way, Streaming Museum curated the project centrally but distributed it through a network of organizations, sites, and screens (both public and private, permanent and temporary). By cultivating an art audience in the interstices between commercial messages, Streaming Museum does not necessarily critique spectacle; instead it seeks ways in which spectacle can be redirected towards experimental aesthetic experiences in public space.

As part of the Nordic Outbreak program, Streaming Museum leveraged its global connections and partnerships to display Icelandic pop signer Björk's Mutual Core (2012) on seven continents. Created by MOCAtv for the Los Angeles Museum of Contemporary Art and directed by Andrew Thomas Huang, Björk's video explores the relationship between the human condition and nature on a planetary scale. In the video, after some energetic struggling and explosions, we see Björk emerging from rocks and sand, and then merging with these animated objects and magma flows. While the "mutual core" she speaks of also alludes to interpersonal connection, the metaphors of "tectonic plates" and eruptions that disrupt stagnation clearly evoke a sense of intraplanetary connection that is anchored to an ongoing negotiation of humans with geographic and geological space. This theme of the merging of disparate spaces, of "ridge drifts to counteract distance" (Björk, 2011), found a match in the curatorial program of Streaming Museum that placed Mutual Core on display in seven continents through projections and public screenings, including an event at the only cinema in Antarctica. Screenings were also held on the BBC Big Screens, a network of public screens across the UK built originally to present the Olympics and Paralympics but also designed to display some artistic and cultural content thereafter. The centerpiece of 
this coordinated event was the Midnight Moment launch at Times Square which spread the video across 15 of the largest screens at the site. Overall, the Mutual Core project exemplified Streaming Museum's opportunistic interstitial approach in order to maximize reach and visibility. Mutual Core speaks to the notion that we must continue to develop empathy to solve social and globally relevant problems through a communicative process.

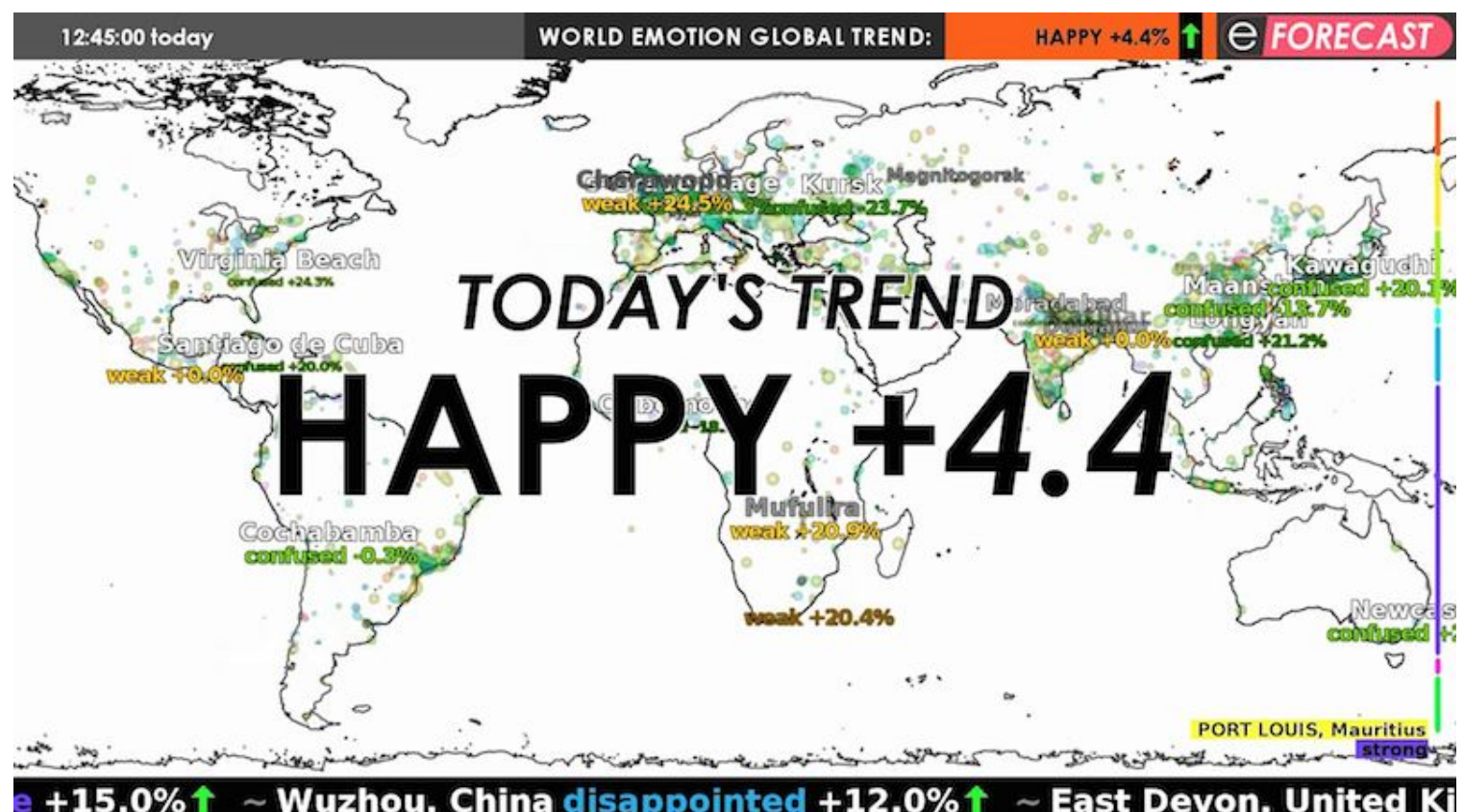

Figure 51. Maurice Benayoun, Emotion Forecast (2012), screenshot. Courtesy Streaming Museum. 


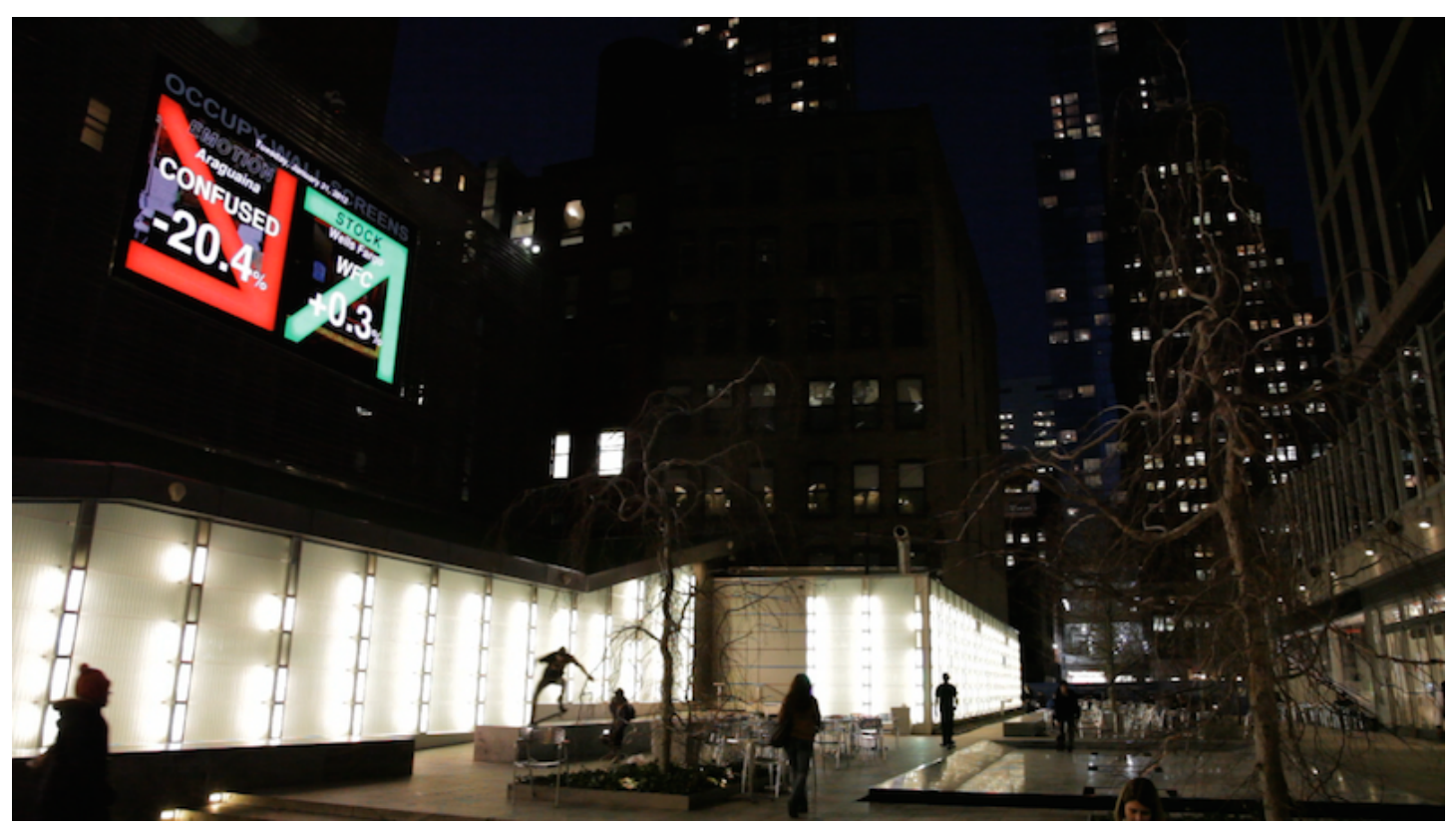

Figure 52. Maurice Benayoun, Occupy Wall Screens (2012), Big Screen Plaza, New York City. Photo: David Bates, Jr., courtesy Streaming Museum.

Streaming Museum engages the global "streaming" of data in relational spaces that merge the logic of space and media. This is further developed in their presentation of French media artist Maurice Benayoun, whose works expose the contrast between omnipresence and site-specificity. Emotion Forecast (2010), a data visualization using the graphics of stock market updates to express real-time changes in the "world emotion global trend," was derived from current events websites in more than 3,200 cities and was shown in New York City's Big Screen Plaza. A second piece, Occupy Wall Screens (2011), was also displayed in Big Screen Plaza, but also in a mix of indoor, outdoor, commercial, and gallery venues including the ZERO1 San Jose Biennial and Federation Square in Melbourne (see Figure 51 and Figure 52). This version of Benayoun's emotional forecasting focused on cities involved in the Occupy Wall Street movement and compared their civic mood with the market trends of financial stocks. As the artist notes, Emotional Forecast could exist 
anywhere, as it was created from and for the Internet. It could be widely sourced and distributed for screens on desks and walls alike, and thus fit seamlessly into most display contexts in a globalized world. On the other hand, Occupy Wall Screens reflected the importance for some media work to be context specific-in this case, placed in a public square in New York City, allowing the energy surrounding the Occupy movement to stream back into its core. Occupy Wall Screens engaged in the spectacle created by the globally recognizable images of New York City and the Occupy Wall Street movement by coopting a public screen typically employed to co-construct and amplify this spectacle.

At the same time, Benayoun and Streaming Museum have used the primacy of New York City to their advantage, noting, as the artist does, that New York City is "the place to be when you want something to be heard by everybody" (Toft 2012). By involving the mutually inclusive "mutual cores" of virtual space (embodied by the flows of data and connectivity of the Internet) and a heavily screened, globalized, urban environment (exemplified by New York City in general and Times Square specifically), Streaming Museum presents a model of curation that inserts itself within existing spectacular displays and commercial and artistic streams. Stitching together programs of cosmopolitan public address, they adapt commercialized sites as productive venues for global cultural development. Optimistically, as stated earlier, Colosi claims such platforms can support a "system of global governance" $(2012,52)$ akin to the United Nations where citizens are connected by streaming channels and interconnected media to collectively make decisions on issues of international import. Yet, Benayoun's work for Streaming Museum provides an example of what a component of such a "system of global governance" might look like 
by proposing a way that data and coordinated public visualization might be used to identify the causes and effects of trans-local phenomenon in a globalized world.

The takeover of Times Square for Streaming Museum's Nordic Outbreak and the coordinated global program that followed addressed the use of a composite dispositif by uniting the local with a global network of temporary partnerships, screens and spaces. Nordic Outbreak extends one of Anna McCarthy's ideas that aggregated screen environments (such as the multitude of monitors in the Virgin Megastore, once located in the middle of Times Square) physically and affectively mobilize the spectator into the space of address. The outcome of such coordination is a "public personhood" $(2001,117)$ in which screen placement and protocols shape subjectivity. By emphasizing the oscillation between the personal and communal, local and global, physical and virtual, and commercial and artistic, Streaming Museum expands the parameters of screen cultures to engage viewers in a hybrid mode of address that engages a transversal subjectivity through a composite dispositif.

Streaming Museum believes that a productive balance can be achieved between spectacle and art. In their projects, commercial sites became the places to implement new models for reconceiving the public sphere through art and technology. Colosi argues that corporations should see the inherent value in aligning their image with culture, and vice versa for curatorial organizations. As Colosi (2014) notes, "I don't think about [corporations and commercialism] as factors we're working against." Instead, "the focus is on the realization of mutual benefits." Colosi departs somewhat from the oppositional rhetoric of Connecting Cities, as well as Artangel and Public Art Fund before it, by 
revealing a more cooperative stance toward the sites of massive media that, due to scale and cost, can seldom avoid some form of commercial or touristic justification and may be best tethered and combined through a combination of art and commerce.

\section{Creation-as-Research: Curating the Ryerson Image Arts Building}

As a creation-as-research project focused not on artistic creation but on the curation and cultural programming of massive media, I have planned and proposed a curatorial initiative for the LED façade of the Ryerson Image Arts Building (see Appendix 1). The Ryerson Image Arts Building was the venue of the creation-as-research project mentioned in Chapter 2, In The Air, Tonight, which visualized Twitter activity related to homelessness and local wind speed and direction on the low-resolution LED façade of the building. After presenting this project for a second time in 2015, university officials warmed to the idea of instituting a year-round curatorial program that would build upon the philosophies and frameworks of Connecting Cities and Streaming Museum described in this chapter. In implementing a year-round schedule of lighting events for the LED façade of the Ryerson Image Arts Building, allowing students to propose lighting schemes and create and participate in interactive installations, the building can become a commons, open to local interactivity and to trans-local connections as evidenced by projects such as In The Air, Tonight and E-TOWER. It can become more open and available to the community around it and thus alter the distribution of the sensible in the built environment.

Like many of the venues that Connecting Cities has migrated towards, the Ryerson Image Arts Building, an institution with art and design programs, is an ideal site for 
experimentation and for the development of an audience that can understand and appreciate the work presented on it. This can occur through the long-term habituation and exposure that is possible with a permanent exhibition and interaction site. Over the course of a number of years, students and residents in the area will be exposed to various modes of expression and interactivity on the building, learning where and how to decode and interact with the building, and proposing their own uses.

In order to support such programming and the development of an informed and receptive public for the building, I have proposed a technical framework similar to the one created by Connecting Cities to allow for an ease of programming by artists and students. I have also proposed to design and install a permanent interactive public terminal and dedicated seating to further augment the possibilities for interaction with the building and entrench it within a composite dispositif of on- and offline spaces that position it as an exhibition space for massive media. Furthermore, promotion, integration, and documentation of the works presented on the building through social media and websites (such as YouTube) will expand the reach, influence, and creative possibilities of the works and reinforce the transversality and post-production affordances of the site. In proposing a permanent exhibition program for this building, I see myself first as an activist, working towards opening up this building to greater expressivity and engagement, and second, similarly to Nina Colosi and Susa Pop, as an interface actor, facilitating artistic experimentation through the provision of technical protocols, support, and institutional partnerships. 


\section{Conclusion: Connecting Sites and Streams}

Massive media can, and most likely will, persist as a highly commercialized

phenomenon, but it can also be pressed into service, through coordinated curatorial efforts, to critique or co-opt commercialization, or to re-envision the role of urban media environments in shaping collective identity, historical consciousness, and public display culture. Connecting Cities and Streaming Museum provide us with two hypotheses for how large-scale public projections and public data visualizations can operate critically, creatively, and cooperatively. Connecting Cities focuses on developing public and curatorial interfaces with existing art organizations that facilitate experimentation and urban prototyping and Streaming Museum provides a curatorial model that seeks to convince both art and non-art venues of the epistemological value of connected public displays and cultural programming on a global scale. These works (as well as my nascent efforts in creating a curatorial program for the Ryerson Image Arts Building) can be seen to embody Maria Lind's (2010) recent arguments about what curating is today. She imagines curating "as a way of thinking in terms of interconnections: linking objects, images, processes, people, locations, histories, and discourses in physical space like an active catalyst, generating twists, turns, and tensions" (63). Connecting Cities aligns itself with existing arts organizations that have access to display sites, emphasizes the importance of the interface between the public and the work in their curatorial selection and direction, and curates as a network which includes providing open-source software repositories for each of their sites. They are primarily concerned with the provision of shared technical information, strengthening partnerships with government funders, networking and sharing 
information amongst presentation sites, and developing audiences, networks of circulation, and cataloguing works. Streaming Museum prefers a mixed approach, connecting with museums, galleries, and art organizations when possible while also developing economic arguments to convince corporate entities, such as Times Square, of the value of global public programming for social justice and the artistic use of big screens for civic and commercial development. Both are concerned with introducing new aesthetic and conceptual ideas into public spaces, commercially owned or otherwise, for the purposes of developing cosmopolitan trans-local and transversal thinking, and developing audiences for these new combinations of space and media.

For both Connecting Cities and Streaming Museum, sustainability, audience development, and the idea of trans-local and transversal activity, connection, and action lies at the heart of addressing the perceived need to connect places that share culture (or are asked to question the integrity of this claim through the embodied telepresence enabled by shared cultural experiences). As such, they begin to renovate ideas of what the commons is and what it means to be public today. It is an idea that is very much rooted in the sense that local contexts must be researched, respected, and developed, while "local" also has a networked meaning in that familiarity and tradition can exist also online, and thus anywhere that it can be accessed and engaged. Colosi argues that trans-locality is communicated by computers, mobile phones, and big screens alike (April 7, 2014, e-mail to the author). Similarly, theorist Tobias Ebsen points out that "Instead of regarding the immaterial content as detached from the material medium, it becomes possible to conceptualize them as interdependent elements" $(2013,166)$. Perhaps the greatest 
contribution Connecting Cities and Streaming Museum is to interrogate the challenges and possibilities of an increasing interdependency and elasticity of contexts, people, technologies, and places: that is, to imagine and begin to construct a hybrid commons with massive media.

Support for massive media-large urban screens, reactive architecture and public projection sites-is becoming more prominent in major cities around the world. The projects of Connecting Cities and Streaming Museum show how curators have redefined the public sphere in the form of networks of public display, distribution, and institutional coordination. Practices at these two organizations demonstrate a broader shift in curatorial attention from autonomous artworks to curatorial networks, hybrid infrastructure, transfer protocols, technical specifications, and software packages. They also demonstrate that largescale urban digital arts require particular tactics to either work with or in opposition to preexisting, commercialized sites of display. Crucial to the curatorial process of massive media are negotiations with corporate and civic entities, each with their own goals that impact what can be presented. Beyond selection and production, curating such sites involves a responsibility for harnessing complex dispositives and the complexities of trans-local space for aesthetic critique and empathetic social connection. 


\section{CHAPTER 5: Conclusion}

This dissertation has focused on emerging combinations of public space, architecture, media, and people centred upon low-resolution media façades such as the tip of the Empire State Building, public projection sites such as the Quartier des spectacles in Montreal, and the many sites of illuminated, urban, public displays, commercial and otherwise, engaged by curatorial groups such as Connecting Cities and Streaming Museum. I have termed these phenomena massive media: they are massive in their size and subsequent visibility, but also an agglomeration of media in their expressive screen and cinema-like qualities and their associated audio, interactive, and network capabilities. I have demonstrated how these assemblages challenge and enable new expressive and narrative capabilities for buildings, cities, and citizens and transform public space, the public sphere, monumentality, and architecture in ways that better represent visual, digital, and public culture today. The impetus for this work has been the proliferation of these heavily screened and often networked architectural environments and the growing impact they have on the development of public culture and architecture. This dissertation has provided a greater creative, historical, and theoretical understanding of massive media, which is vital to the ongoing development of public art and the public sphere. In shaping our understandings of who we are, individually and collectively, and changing how we read and interpret the world around us, massive media have necessitated the development of analytical and practical tools related to their roles in art, politics, and culture.

More specifically, this dissertation explored and elucidated new capabilities and spatial transformations of massive media, situating them historically, exploring them 
further through case studies and, finally, investigating them through creation-as-research in order to both suggest and test fruitful practical and theoretical strategies for their creation, theorization, curation, and critique. The larger aim of this has been to strengthen the theoretical and methodological foundation for this field of study and practice, situating it both within a history of media, architecture, information studies, and urban studies, as well as within a history and practice of public art and a trajectory of curation. In particular, the creation-as-research projects in this dissertation have aimed to operationalize and test some of the theories about space, monumentality, and architecture that I have applied to this emerging field while also operating as a more active and popular form of exposition and exegesis-helping to develop actual audiences, structures, and protocols for public displays of large-scale digital and moving-image based art. Overall, this dissertation proposed tactics for practitioners and researchers in architecture, new media art, and the humanities, establishing critical perspectives, theories, and methods for the practice and critique of public visual culture with a form of media emerging within rapid technological, social, epistemological, ontological, and economic change.

Chapter 1 outlined three theoretical pillars that underpin the stakes and study of massive media. In outlining the first pillar, the public sphere, I discussed the way the concept of the public sphere has changed over time and with media. While the health of a public sphere has been generally predicated upon creating ecologies of communication that are as accessible, autonomous, non-hierarchical, and participatory as possible and lead to consensus over contentious issues (Habermas 1974), the ways in which this has been operationalized has changed from spaces of public assembly, to café culture and 
newspapers, to satellite television, and now to a myriad of fragmented yet interconnected media forms. These changes, over time, have also broadened what we see as the purpose of the public sphere which must now also reflect the apolitical and agnostic (Warner 2005), as well as characteristics of openness, playfulness, emotion, inclusivity, heterogeneity, unpredictability, conflict, contradiction, contestation (Mouffe 2007), and difference (Negt and Kluge in Hansen 1993). These changes in media have also radically changed its spatial, territorial and geopolitical parameters (Negt and Kluge in Hansen 1993). It is within this milieu of the public sphere that I argue massive media emerges as a powerful element within it, an element that acts as a dense transfer point for the fragmented on-and offline publics that we participate in and is thus generative of a new conception of a hybrid “commons" (Hardt 2009).

I also showed massive media to be situated within a history of monumentality. A history of the theory of monuments shows how the predecessors of massive media have been powerful and contested points of reference in the city, but more so, elements that, through displacement and condensation (Lefebvre 1997), swap the many for the one and accrue layers of shared meaning in their singularity and centrality. They have combined the material, symbolic, and functional through their presence and the practices associated with them to become sites of memory (Nora 1989) and connection. Following from this, Giedion and his colleagues at the CIAM identified a deficiency in the relatively staid and static monuments that populated their modern world and sought to shape the future of monuments by arguing that they should be more dynamic and inclusive of participation, better reflecting the presence of people and not just the spectacle of the monument itself. 
They even went as far as suggesting that moving images be incorporated in some way in future monuments (Mumford 2000). In these ways, monumentality, and the debates about it in history, were shown to be central to the theory and practice of massive media.

Finally, and more abstractly, the relationship between space, architecture, and media was demonstrated as an important theoretical pillar for the study of massive media. Building upon Lefebvre's (1991) theories of space not as static but as produced through social and technical processes, I outlined McQuire's (2008) theories of relational space in what he calls the "media city." The imbrication of media and space, through mobile ubiquitous media, urban screens, radio and television frequencies, and so on, have produced spaces that are characterized by their ambivalence and contingency. As such, space and the exchanges within that establish identity and place are seldom unidirectional, homogenous, and fixed as they were once assumed, but instead assume significance through heterogeneous, variable, and impermanent interconnections (22). It is within this hybrid space (De Souza e Silva 2006, 261) that massive media emerge as architectural elements that produce and harness this ambivalence and contingency and produce accelerated rituals that redefine monumentality and the public sphere, both dehistoricizing and potentially rehistoricizing a space through the engagement of an affective register identified in early studies of the moving image (Kracauer 1965). Following also from film studies, massive media can also be seen to engage a composite dispostif (Verhoeff 2012), that is a state of mediation that combines standardized environments of spectatorship (i.e., mobile phones, moving images, radio, architecture) to recenter an audience to a specific public address. Such theories and practices are also reflected in theories of 
supermodernism (Ibelings 2002) in architecture, a term that describes the growing influence of digital culture in the design, creation, and reception of architecture. Massive media can be seen as both an expression and a growing development of a potentially new architectural and cultural epoch.

Building upon these theoretical pillars, Chapter 2 investigated the narrative and associative potential of massive media with respect to moving images, focusing primarily on Robert Lepage's The Image Mill (2008-12), McLarena (2014) at the Quartier des spectacles, and two creation-as-research projects, 30 moons many hands (2013) and The Line (2013). Techniques specific to the moving image such as superimposition, montage, dispositif, and participation were analyzed for their transformations in massive media and their role in creating relational spaces, new forms of public spheres, and enacting a "new monumentality" (Mumford 2000, 151). These techniques of cinema proved to be useful analytical tools for understanding how massive media compares to cinema, but also how it functions beyond the affective registers and limits of cinema. For example, the spatial, extra-diegetic, and mediatic superimpositions in the composite dispositif and montages of massive media represent an ontological blurring between diegesis and the space surrounding it, enacting what amounts to a kind of "creative geography" (Kracauer 1965, 48) that effectively blurs time, space, and context. Spatial montage and superimposition contribute to a framelessness and spatial ambivalence characteristic of this new media form, enabling new possibilities for narrative and association between the city, people, and the moving image. Furthermore, the ability to view a work from radically different perspectives and in varied states of attention and distraction by virtue of an incomplete, 
always-composite montage, as well as the ability to participate in public visual culture at the scale of architecture, position massive media as generative of a politics of aesthetics (Rancière 2000), creating productive artistic equivalents to real political positions similar to the way that Bayer's Family of Man created a "democratic surround” (Turner 2013). In particular, McLarena, in its openness to participation and remediation, can be seen as constructing a new democratic surround, a public sphere for relational space enacted through public art, making good on Giedion's call for a "new monumentality" by allowing people to play a central role in reanimating history (in this case, the works and legacy of filmmaker Norman McLaren) in a joyful event of remembrance and collectivity. Specifically, at a time when public culture is seen to have either retreated to the disembodied, heavily surveilled and standardized confines of the web, or to have been fragmented by media saturation and mystification in material public spaces, the examples covered in this chapter posited promising habit and identity formations in new venues for a public sphere in a digital age. The analysis of McLarena and The Image Mill demonstrate that media in general, and media in public space specifically, need not necessarily estrange us from the terrestrial plane as Virilio (1986) and Crary (1999) warn, dehistoricizing space and alienating those within it. Instead it can show space to be processual, relational, malleable, and personal by harnessing and enhancing its ambivalence and contingency through the clever combination of media and architecture. Critical and creative uses of public projection at the scale of architecture militate against the closure of memory in space and open up a future in which we can use the expanding mutable, oneiric, networked, and expressive surfaces of the city for accelerated rituals of collective 
embodiment and shared memory. That said, as discovered through creating works for this medium myself, the success of a large-scale public address that is both critical and creative depends on a number of factors which include the artist's engagement with a site and the media associated with it, and the willingness of governments to support autonomous creative works and develop long-term commitments to experimentation with massive media. In these ideal instances, engaged audiences can be developed and the spaces around the screens-a crucial element in merging architecture, monumentality, audience, and media-can be properly designed to help entrench the ephemeral enunciations of massive media within a space in memory and culture.

Chapter 3 shifted the focus to a particular set of massive media: large-scale media façades, otherwise known as media architecture, and the public data visualizations they enable. I explained that programmable low-resolution LED displays, such as the tip of the Empire State Building or the lights on Toronto's CN Tower, while exemplifying many of the characteristics of contingency and ambivalence seen in public projection, are often more visible because of the scales and heights they can reach while subsequently less detailed in their display capabilities. They are thus better suited to the display of chart-like data and dynamic visualizations of data from various sources as well as large-scale interactivity through composite media forms such as social media. They are also more directly integrated into architecture and as such are better representations of supermodernism, that is, of architecture characterized by the irruption and imbrication of the "infoscape" and the cityscape (Ibelings 2002). As public data visualizations (Colangelo and Davila 2011), these buildings represent more appropriate outlets for the massive public 
spheres that persist and mutate online, an opportunity to make coherent and collective an expression of the growing importance of data in public culture. Buildings like the Empire State Building, through the addition of expressive architectural lighting, become communicative beacons that capture attention through contingency and context-specific relevance as well as through social media integration, introducing and enhancing elements of ambiguity, expressivity, and participation into practices and places of monumentality. At the same time, the urban spectacle created and harnessed in these forms is often anchored to promotional campaigns (such as \#WhosGonnaWin, Verison's participatory promotional event for the Super Bowl) that use the syncretic power of monumentality, along with the attraction of scale, ambivalence, and contingency (often enacted through participation), making these sites advanced venues for advertising and thus also for the struggle for public culture and aesthetic justice in cities. Tactics for the effective use of such media façades as public data visualizations were outlined in this chapter and evaluated against the criteria set out by Vande Moere and Wouters (2012) and Vande Moere and Hill (2012), namely a sensitivity to the environment, content, and carrier of massive media, and their ability to be situated, informative, and functional. These characteristics were shown to be effective analytical tools for public data visualizations. Extending the analysis, I showed that all of these characteristics were highly dependent on the imbrication of media such as social networks and "post-production" (Steyerl quoted in Shental 2013), that is, the remediation of traces related to public data visualization through elements such as personal photography and social networks. These trace elements require greater scrutiny and consideration when analyzing and creating large-scale public data visualizations. Public data 
visualizations on large-scale low-resolution façades were further explored in creation-asresearch projects with the $\mathrm{CN}$ Tower and the Ryerson Image Arts Building. Both of these projects built upon the idea of a hybrid public as a site of discursive contestation that merged the originary public of concentrated visibility and assembly with the fragmented, asynchronous feedback mechanisms of a transversal (Murphie 2004) public made apparent through networked media and the platform of massive media. That said, in creating public data visualizations and comparing this to the analysis of existing visualizations and scenarios of massive media, it became clear that key concerns for the development of this medium as a venue to engage citizens in important civic issues hinged upon issues of access and moderation. Due to their degree of complexity and the sheer cost of installing LED façades, centralized censorship and control remain a critical factor. Often, the ownership of a building greatly influences what can and cannot be said with and on the building, with buildings like the Empire State Building straddling a fine line between operating as an independent commercial entity, essentially selling ad space on its façade, and as a public, iconic symbol of the city and its people. Similarly, difficulties arose in the negotiation with the management of the federally owned $\mathrm{CN}$ Tower and a compromise had to be reached with them that diverted us from exploring visualizations that could have been interpreted as detrimental to their reputation. Like public projection-albeit perhaps more difficult in the cost and relative permanence of the infrastructure behind the façades-one of the most important roles for artists working with massive media is in gaining access to these sites and the installation of long-term, sustainable programming that can help to develop an audience and culture that is willing and able to explore issues of common concern through 
a composite dispositif centred upon low-resolution expressive architectural displays. Like the massive media of large-scale public projection, low-resolution media façades are semiotic resources for socially engaged practices and urban activism provided proper permissions are negotiated, audiences are sufficiently fostered and developed, and consideration is given to equitable representation through interface design and context sensitivity. Overall, buildings with media façades, more than large-scale public projection sites, function as durable transfer points for digital culture, emotion, and the identification of diverse issues and groups. Thus, they represent an approach to a new monumentality that can reinvigorate mass cultural, communal, and collective experience.

The theories and concerns raised in Chapters 2 and 3 culminated in Chapter 4 which investigated the role that curation has played historically and can play today in new media art in general and massive media specifically to develop the critical and creative use of large-scale public displays. I focused on two case studies, Connecting Cities and Streaming Museum, two organizations with different approaches to engaging urban screens and flows of media with public art. The coordinated efforts of these organizations serve two key functions: 1) they are able to leverage local and national governments for funding, and 2) they can use this funding to create forms, on- and offline, for the sharing and combining of curatorial expertise across dispersed sites of massive media. Finally, through sustained programming, they are able to nurture audiences and develop their tastes and capacities for engaging with large-scale and sometimes interactive and interconnected public works. Through the experimentation and development these sustained scenarios afford them, Connecting Cities and Streaming Museum are able to ask and answer questions with 
massive media, namely: what or where exactly is the space of contestation that we share as urban, networked beings? And to what degree can we see ourselves reflected back to ourselves in our trans-local spaces? Essentially, they ask questions of monumentality and the public sphere in an age of networked communication and the proliferation of mutable screens and surfaces. Installations such as Binoculars to... Binoculars from..., part of the Connecting Cities program, Streaming Museum's coordinated global presentation of Björk's Mutual Core, and massive media in general, begin to provide answers: the space of contestation that we share is a hybrid one that requires a sensitive approach to the combination, presentation, and accessibility of material and information. The degree to which we can see ourselves and our concerns reflected back to us is modulated by these spaces that allow us to participate in a sense of communal importance through contemporary rituals of on- and offline performance. In this sense, both the artist and curator, in their understanding of their role as translator or interface between established structures and art forms (Graham and Cook 2010), harbour a responsibility to create rich spaces of contestation and interaction that blend networked and trans-local participation, once again approximating new political positions for an increasingly cosmopolitan world that requires models of state-less, urban-to-urban representation and communication to solve global problems such as poverty and environmental degradation. To achieve this, Connecting Cities was shown to build upon existing art and new media art organizations that are concerned with fostering artists and audiences instead of grappling with commercial sites that, while often having greater visibility and thus being more "public," can bury art deep within overpowering commercial messages, providing only a sliver of 
space and time every day. Streaming Museum, following from the work of Toronto's Public Access and New York's Public Art Fund, instead seeks to insert their work into spaces of commercial discourse in order to scramble these spaces semiotically, creating a disruption in the regularly scheduled consumerist spectacle in order to deliver artistic messages as well as to cause a disruption which is meant to encourage audiences to reflect on the lack of heterogeneity, conflict, and contestation engaged through large public displays. Both of these tactics are useful and necessary in re-envisioning the role of urban media environments in shaping collective identity and public display culture amidst the persistence of commercialization that dominates-and most likely will continue to dominate-massive media. I concluded this chapter with a creation-as-research project that I continue to pursue with Ryerson University in which I have proposed that the LED façade of the Ryerson Image Arts Building be treated as an exhibition space for art and community engagement. I outlined a number of principles gleaned from the research conducted in this chapter, specifically, the development of technical support systems that allow the community to propose and implement various uses for the media façade, the attention to the development of the space around the façade in order to increase the expressive possibilities for the composite dispositif centred upon the building, and the subsequent development of audiences through sustained, experimental programming on the façade. 


\section{Contributions: Tactics and Responsibilities}

This dissertation presented a number of useful tactics for practitioners and researchers in new media art and the humanities interested in the study of the proliferation of media in public space. One such tactic is the application of analytical tools from cinema studies, namely superimposition, montage, and apparatus/dispositif, to the study of massive media. These techniques and analytical lenses serve to demonstrate the ways in which massive media both follows from, incorporates, and departs from cinema and other standardized forms of technical expression and spectatorship. It also helped to situate massive media within media studies.

This project also explored the application of principles of new media art curation to massive media. I have identified sites of massive media as dense transfer points for memory, identity, commerce, and culture, and thus important sites for sustained attention and critique by artists and designers. Due to their hybrid nature, connected as they are to various sensors, data streams, and remediated through the post-production of personal photography and associated social media networks, massive media require high-level coordination and the provision of technical support from curatorial groups that must see their role as public space activists, negotiating for sustained access to these highly charged spaces, and as educators, habituating audiences to these new forms of contestation and connection and developing more nuanced forms of communication with and through them.

This dissertation also contributed to the ongoing interdisciplinary study of digital culture in its tests of the theoretical claims made about the transformative properties of 
digital technology in cities and for architecture and monuments. The combination of programmable LED façades or digital projection mapping, coordinated sound, and other digitally coordinated media elements such as radio signals and social media, were shown to contribute to theories of relational and hybrid space made by McQuire and De Souza e Silva, while also proving productive for the engagement of a new approach to architecture and monumentality that we might describe as "supermodern" (Ibelings 2002) which sees structures as imbricated and expressive of the participatory, contingent, and ambivalent nature of expressions and connections that we experience in a data society. The connections made between people, images, information, and space do not displace tradition, connection, and meaning so much as give it a different velocity and topography. Armed with this knowledge, we might reliably predict and guide future outcomes, directions, and critical practice in the growing field of massive media and the ever-evolving fields of architecture, social media, public space, and monumentality. One such practice that this dissertation exposes and outlines is that of public data visualization which is particularly prevalent in large-scale low-resolution media façades. Public data visualizations, in their massive embodiment of data of shared consequence, and the subsequent intensity of embodiment experienced by spectators and participants against the scale and significance of urban space, are poised to grow in number and importance, and thus must continue to be analyzed and developed critically. This can be done, as seen here, through social media analysis which exposes the uses of public data visualizations at a micro and macro level, dissecting individual social media posts and discourses that demonstrate levels of contestation and conversation centred upon massive media, but also by observing their 
overall representative usage over time. It can also be done by carefully analyzing and considering the context (carrier, content, and environment) (Vande Moere and Wouters 2012) of massive public displays, and evaluating how they are situated, informative and functional (Vande Moere and Hill 2012).

Finally, in several creation-as-research (Chapman and Sawchuk 2012) projects, this dissertation demonstrated the value of an engaged practice with massive media to better reflect on the ever-changing qualities and characteristics of agglomerations of space and media, and to disseminate theories and shape public culture more directly and publicly. By working directly with the materials and theories of space, media, and monumentality, I was able to better understand the importance of developing a composite dispositif through various on- and offline means to capture and deliver an audience to experiences with space, history, and one another that were playful, participatory, heterogeneous, inclusive, unpredictable, and contradictory-in short, I was able to operationalize a public sphere centred upon massive media and reflect upon its related theories and outcomes. These experimentations, particularly in the effort involved in producing them and the struggle to extend their presentation to have a lasting, meaningful impact on an audience, also demonstrated the importance of the development of curatorial programs for these spaces as well as purpose-built, well-supported, dedicated sites for experimentation with trans-local encounters through massive media that serve to develop the complexities of a new public sphere that will be necessary for solving the complex trans-local issues of identity, environment, and economy that we will continue to face. 
Overall, this dissertation has developed specific practical, theoretical, and methodological lenses that serve to develop a new field of study in massive media and situate it within interdisciplinary fields of digital humanities, architecture, urban studies, art history, and media studies.

\section{Recommendations and Future Directions: More Massive, More Media}

More study and action is needed by artists, curators, and academics in the field of massive media. For one, technologies, tools, and practices in the field are changing every day, with bigger, brighter, and lighter lighting elements and projectors being produced, mounted, and directed at wider swathes of the urban environment. In addition to this, improvements and developments in the composite media forms of massive media such as social media and embedded, networked sensors are undergoing similarly rapid changes and evolutions as evidenced by the growing importance of the Internet of Things (IoT) which seeks to create networks of sensitive, communicative objects through embedded electronics. As massive media grows in its applications and complexities, we must continue to probe it for its critical potential and its ability to build empathy, connection, and context across on- and offline spaces, developing publics to serve trans-local identity formation and cultural expression. Arts organizations, universities, curatorial agencies, and governments at all levels must appreciate and understand the growing importance of these sites for this purpose.

With this in mind, and having recently returned from a Media Architecture Summit in China, there is a great deal of potential for future research and practice in the 
growing and multiplying cities of Asia. Specifically, I was struck by the level of coordination and scale of massive media installations in China, with entire cities featuring coordinated low-resolution lighting displays that from a distance resolve into choreographed animations serving to entertain citizens and brand the city because of (and in spite of) their relative anonymity caused by the speed and standardization of construction. For example, cities that may have once been important ports would feature stylized sails or depictions of a certain style of architecture (once) characteristic of the region across these urban-scale media façades spread over roughly twenty buildings. Of particular importance for further research in this area is the role that documentation plays in producing an image of the city through massive media. While the Empire State Building populates its social media feeds nightly with high-quality images taken from an ideal viewpoint, these extra-large-scale displays are captured in their entirety from vantage points that are shared by few in the city, but widely disseminated and potentially internalized by larger, networked populations to produce a collective image of the city primarily through remediation. Of equal importance here is the need to extend research to other countries and regions, comparing the role that regional politics and culture play in shaping the kinds of massive media and practices associated with them. McQuire and his colleagues (Barikin et al., 2014) have already pioneered some of this research by studying the translation of gestures between large public displays in Seoul and Melbourne, but much more research is needed as these scenarios and practices continue to proliferate.

Another area in which this analysis might be extended using the tools and methods presented in this dissertation is in taking a closer look at the specific differences between 
the use of live and pre-recorded images in large-scale public artworks. Although I have covered a number of massive media scenarios in this dissertation, I have not specifically focussed on the qualities of live-ness in the creation of powerful emotional and collective experiences. Yet another area to explore is the prevalence of the use of the face in massive media to communicate, connect, and develop empathy. More study into the history of the face in monuments and in architecture may produce interesting insights into the contemporary use of portraiture and selfies in massive media. More research could also be done into the ways public art has addressed specific societal issues and how it can now be addressed differently or more effectively through massive media. To focus this analysis, a single issue could be selected, such as climate change or poverty, and case studies could be presented and analysed along with a number of creation-as-research projects. A particularly promising case study for such an investigation might be the ArtCOP21 event planned for December of 2015. Part of COP21-the $21^{\text {st }}$ United Nations conference on climate change-ArtCOP21 has been planned as a parallel event to stage city-wide cultural events that "address climate as a people challenge" (ArtCOP21), not just a scientific conundrum. The program promises to include "monumental art installation in significant public spaces" (ArtCop21).

Finally, more study is needed on existing organizations, their protocols, practices, tactics, and results with respect to massive media. The innovative work of organizations such as Medialab Prado and Galeria de Arte Digital SESI-SP in Sao Paulo have yet to be studied in greater detail and would make excellent case studies to answer ongoing questions about the provision and programming of massive media: How are publics 
continuing to engage with massive media? How can their feedback be solicited and integrated into the planning and production of massive media? Do citizens have the opportunity or right to decide if their cities will be the sites of such agglomerations of media and architecture? And if so, what level of control do they have over their programming? I have started to explore these questions in this dissertation, but more work is needed as massive media become more massive in their size, reach, and consequence for the future of public culture. 


\section{APPENDIX 1}

Ryerson Image Arts Building Proposal

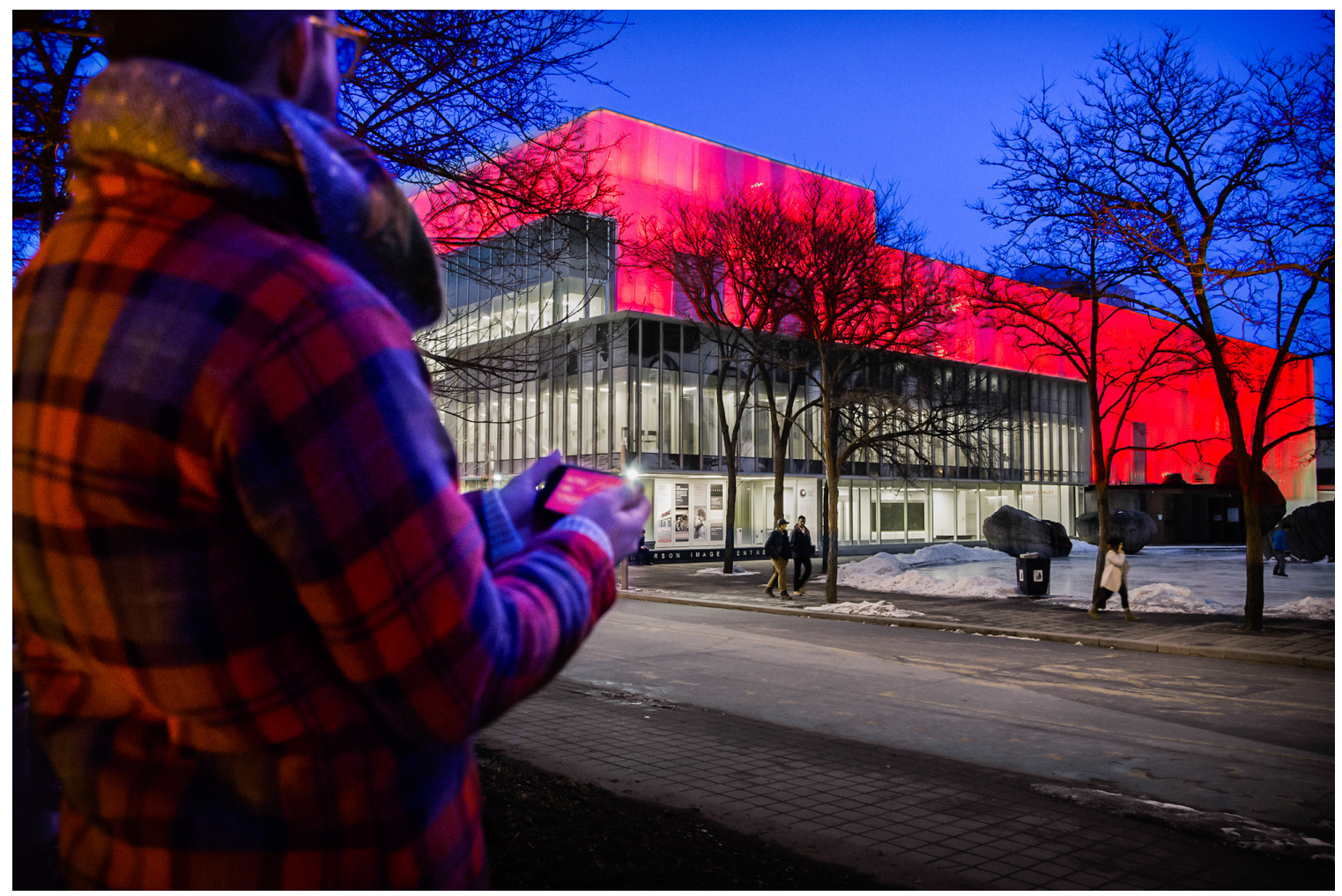

In The Air Tonight (2014), Dave Colangelo and Patricio Davila

\section{Proposal}

\section{Engaging the Ryerson Image Arts Building LED Façade January 2015}

\section{Dave Colangelo}

Media Artist and Consultant

PhD (ABD), Communication and Culture, Ryerson University

david.colangelo@ryerson.ca

http://davecolangelo.com 
To implement a year-round schedule of lighting events for the LED façade of the Ryerson Image Arts Building.

Like the CN Tower, the Empire State Building, or the ARS Electronica Center, the award winning LED façade of the Ryerson Image Arts Building can be used as a dynamic focal point for community building and artistic experimentation. It can be programmed to reflect city-wide events, holidays, and special lighting requests, and used as an exhibition space for digital art by students, alumni, and world-class artists. Promoted, 'liked', and shared on social media, a year-round,

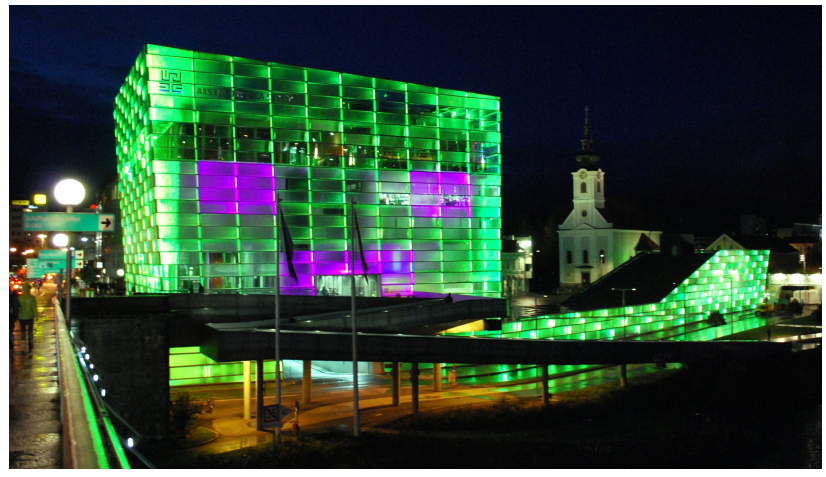

Ars Electronica Center, Linz, Austria.

coordinated lighting program for the Ryerson Image Arts Building can generate powerful connections between students, the school, and the city.

To create exceptional experiences with the LED façade of the Ryerson Image Arts Building for students and citizens, and to promote Ryerson University as a dynamic, innovative, diverse, and inclusive city-building institution at the forefront of media arts and culture.

In summary, Ryerson University should:

- Engage in long-term infrastructure maintenance and improvements to make the most out of this valuable and unique asset.

- Implement a year-round program of lighting events to reflect holidays, special events, and causes, promoting these events through social and traditional media;

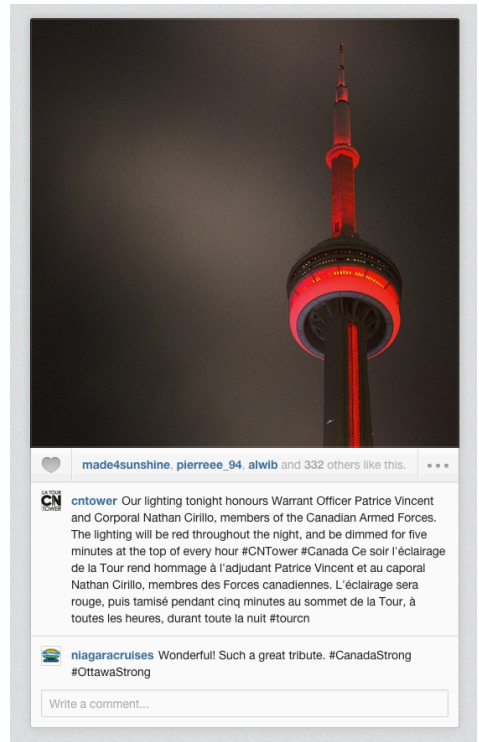

CN Tower on Instagram.

- Facilitate the curation and coordination of a program of media art installations on the façade, inviting students, staff, alumni, and world-class media artists to work with the façade;

\section{Dynamic Uses and Functions of the LED façade}

The LED façade of the Ryerson Image Arts Building can produce dynamic graphic and ambient effects in millions of colours. In addition to this, the fully-programmable system can be linked to data streams and sensors to enable interactive uses of the façade and dynamic data visualizations. 
Holidays and Special Occasions (year-round)

Lighting effects and colours can be programmed to reflect special occasions throughout the year including Christmas, Hanukah, Eid, Diwali, Valentine's Day, Pride, Thanksgiving, etc.

On and Off Campus Events (year-round)

School colours and special animations can be featured to promote key sporting events. Lighting schemes can be used to reflect colours of visiting delegations. Moreover, the lights may be coordinated to coincide with opening events at the Ryerson Image Centre (RIC) or city-wide cultural events such as Luminato, Tiff, or Nuit Blanche.

Teaching and Research (3-4 weeks / year)

Interactive and dynamic uses of the façade can be explored across disciplines by students, faculty, and alumni in the Faculty of Engineering \& Architecture, Communication \& Design, and the Digital Media Zone.

World-Class Media Art Installations (3-4 weeks / year)

Similar to the ARS Electronic Center, the LED façade of the Ryerson Image Arts Building can be used as an exhibition space for groundbreaking media art installations. Potential high-profile artists include Rafael Lozano-Hemmer, Olafur Eliasson, and Alfredo Jaar.

Social Media (year-round)

Social media profiles (Twitter, Facebook, Instagram), similar to those employed by the Empire State Building or the CN Tower, can create a conduit for students and citizens to share information and images related to Ryerson University and the building's lighting program.

(Optional) Permanent Interactive Public Terminal and Public Furniture (year-round)

The installation of a dedicated terminal and interaction space (with public furniture such as benches, lighting, and speakers) on the east side of Lake Devo can help to make the most of the building. This will allow people to learn about the lighting program, congregate more comfortably, and interact with the building in new and exciting ways.

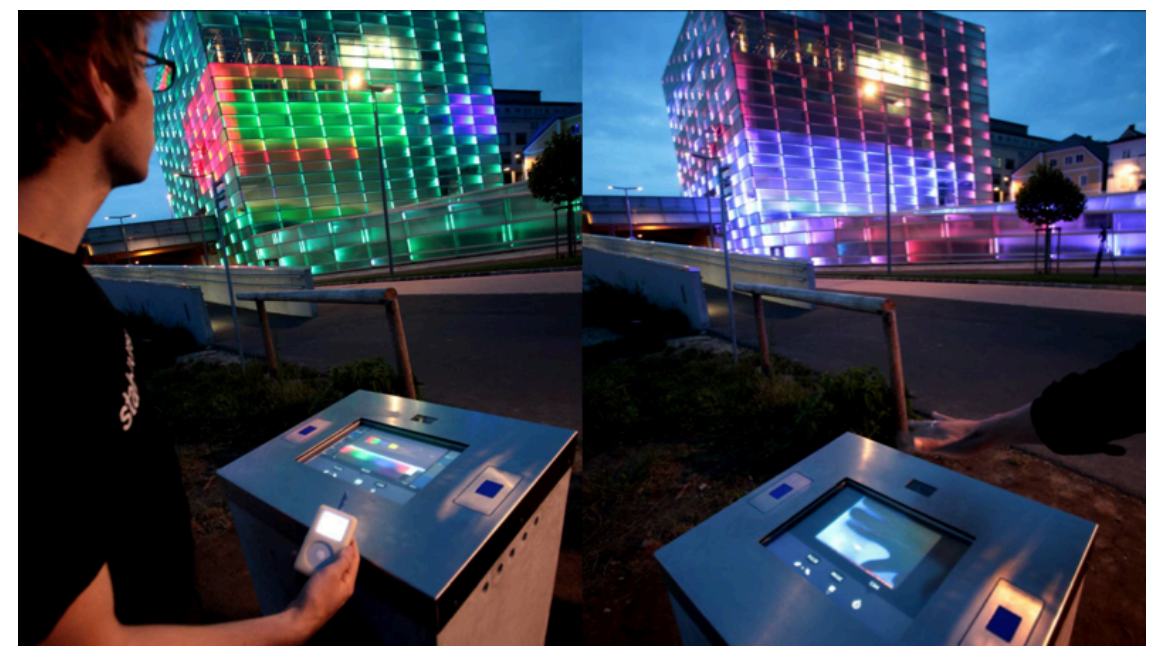

Façade terminal at the Ars Electronica Center. 
- Lighting Program Management

- Create process for special lighting requests for on and off campus groups.

- Engage student groups and faculties to create opportunities for learning and engagement.

- Communicate and coordinate lighting schedule with University Advancement, President's Office, Ryerson Image Centre.

- Manage and monitor nightly

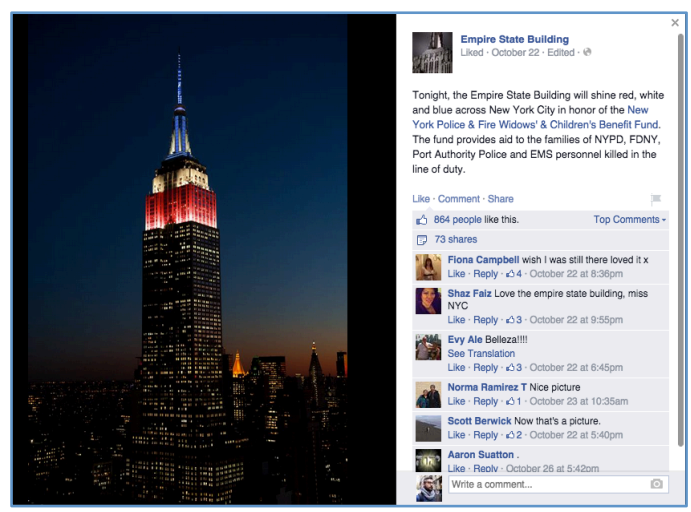

Empire State Building on Facebook. lighting program.

- Marketing and Promotion

- Create and maintain social media presence to promote community engagement with lighting programs and events.

- Interface Maintenance and Support

- Manage and track maintenance of software and hardware components.

- Provide up-to-date technical information and support for artists, students, etc.

- Curation

- Formation of jury to extend call for proposals and select media artists.

- Manage artists and launch events.

- Apply for arts council funding for special projects and artist residencies.

- Infrastructure Maintenance, Coordination, and Planning

- Establish "baseline" for lighting system physical infrastructure (Campus Facilities \& Sustainability). (Critical)

- Install web-cam to remotely monitor lighting program. (Optional)

- Install interactive terminal and public furniture on west side of Lake Devo.

(Optional)

Projected Yearly Expenses and Funding Sources

\section{Expenses}

$\$ 40,000 /$ year

$\$ 20,000 /$ year

$\$ 10,000 /$ year

$\$ 5,000 /$ year
Coordination, promotion, and curation of LED façade lighting program

Artist Residency for high-profile media artist

Infrastructure and Maintenance Costs

Design, printing, signage, event costs, promotional materials

\section{Funding}

$\$ 20,000 /$ year

$\$ 40,000 /$ year

Canada Council for the Arts - Funds for New Media Artists

$\$ 15,000 /$ year

Sponsorship and Naming Rights (ie. "Sponsor's Name New Media Façade")

Ryerson University

\section{Optional Expenses}

$\$ 24,000$

Design and installation of public computer terminal 
$\$ 15,000 \quad$ Design and installation of public furniture

$\$ 1,000 \quad$ Webcam installation to monitor LED façade

History of the Ryerson Image Arts Building LED Façade

The Ryerson Image Centre and School of Image Arts was completed in September, 2012.

Designed by Diamond Schmitt Architects, the building includes a fully programmable wraparound low-resolution LED façade of variable dimensions consisting of $7273^{\prime} \times 8^{\prime}$ backlit translucent glass panels. The cost of the lighting system was $\$ 3$ million of the total $\$ 61$ million construction cost.

2012 Control of LED façade shared by Ryerson Image Centre and University Advancement. Artist-in-Residence David Rokeby and New Media Faculty David Bouchard develop System Overview, LED Map, and User Manual, and Real-Time Interface for LED façade. Architectural Lighting Magazine, Light in Architecture Awards - Best Use of Color

2013 Control of LED façade transferred to University Advancement. Design Excellence Award from OAA, SCUP/AIA-CAZE Excellence in Architecture for Building Additions or Adaptive Reuse Honor Award.

2014 In The Air, Tonight installation by Dave Colangelo and Patricio Davila, first large-scale interactive use of LED façade. Project included in 2014 Media Architecture Biennale.

\section{About - Dave Colangelo}

Media Artist - http://davecolangelo.com

I create public installations involving architectural LED façades and projection. E-TOWER (2010) engaged the CN Tower's LED façade by programming it to change colours based on the frequency of text messages during Nuit Blanche. In The Air, Tonight (2014) used the LED façade of the Ryerson Image Arts Building to raise awareness about homelessness by having the building glow with each instance of the hashtag \#homelessness on Twitter. The project was picked up by numerous media outlets and was featured on an episode of CBC Radio's 'Spark'.

Independent Consultant and Researcher

I advise organizations (OCAD University, Bortolotto Architects, Connecting Cities, Public Art Lab) on best practices related to the selection, installation, and use of expressive architectural systems such a LED façades and permanent public projection sites.

\section{Media Scholar and Educator}

I teach and write about media, digital culture, and public space. My PhD (Ryerson, 2015, draft submitted) thesis, entitled "Massive Media: Expressive Architectural Surfaces and the City," investigates urban screens, LED façades, and public projection as spaces for creative engagement between the city, public space, and information. I also hold an MA in Cultural Studies and Interactive Media from Goldsmiths College, University of London. 


\section{APPENDIX 2}

\section{Letter Regarding Originality of Dissertation}

\section{Dave Colangelo}

davecolangelo.com, david.colangelo@ryerson.ca

1257 Dundas Street West, Toronto, ON, M6J 1X6, 416.836.2075

June 15, 2015

This letter describes the nature of my collaborations in the production of four creation-as-research works described in my dissertation, "Massive Media: Theories and Practices of Large-Scale Projections and Public Data Visualizations," presented to Ryerson University and York University in partial fulfillment of the requirements for the degree of Doctor of Philosophy in the Program of Communication and Culture.

E-TOWER (2010) was a collaboration between myself and Patricio Davila. For this project, I was responsible for the initial concept and proposal which was accepted by the Nuit Blanche, Toronto 2010 committee. The concept was further developed between myself and Davila and we jointly secured permission from the CN Tower and sponsorship from Rogers Communications to pay for production costs. Davila and I were both involved in developing the final concept as well as in production meetings with Rogers, Nuit Blanche, and the CN Tower. Davila and I worked iteratively throughout, with Davila taking on most of the programming duties while I assumed most of the production duties including web design. We received in-kind assistance from graphic designers Berkeley Poole (flyers) and Jamie Webster (tower animations), as well as in-kind documentation support from Udo Dengler (time-lapse video) and Sarah Lasch (promotional video).

30 moons many hands (2013) was a collaboration between myself Patricio Davila. We were first introduced to the Archives of Ontario by Dr. Janine Marchessault at York University. Davila and I carried out research and documentation at the Archives of Ontario with the support of archivist Sean Smith. I was involved in storyboarding, video production, audio recording, web design, and initial video editing. Davila was responsible for video production, editing, and projection mapping. Both Davila and I were involved in the final production of the installation. We received in-kind assistance from graphic designer Patricia Davila (poster), photography assistance from Jamie Webster (documentation photos), and the in-kind donation of the use of two 10k projectors from York University's Sensorium: Centre for Digital Arts \& Technology.

The Line (2013) was a collaboration between myself and Patricio Davila. It was part of a group exhibition entitled Land/Slide: Possible Futures at the Markham Museum in Markham, Ontario, curated by Dr. Janine Marchessault. Davila and I were involved in early conceptual meetings with Marchessault and other artists and curators. Davila and I were equally involved in the concept, design, and planning of the installation as well as in the construction of installation materials and the production of the sound and video elements of the piece. Davila's time was mostly spent creating and editing the final video while my time was directed towards logistical and production concerns at the site. We received in-kind assistance from photographer Will Pemulis (photographic documentation), construction assistance from Massimo Di Ciano, Adam Colangelo, and Lucas 
Cesare Brancalion, and an in-kind donation of the use of two 10k projectors from York University's Sensorium: Centre for Digital Arts \& Technology.

Finally, In The Air, Tonight (2014-) was a collaboration between myself Patricio Davila. Initial conceptualization and negotiations with the administration of the Ryerson Image Arts Building LED façade were made by myself, Jon Friis, and Takin Aghdashloo in 2011. After Friis and Aghdashloo left the project, Davila joined and we jointly refined the concept for the project, raised funds, and put together a team to complete the project. I was involved in initial programming, building animation design, and computer modeling. Davila hired and directed Robert Tu in completing the programming for the back-end of the installation (php and Processing) while Maggie Chan was hired to complete the building animations and produce documentation of the month-long installation. The team shared various production duties including refining the concept, installing a remote weather station on the roof of the building, installing a webcam, producing promotional materials, moderating the Twitter feed, and public relations planning and execution. In-kind consultation and support was provided by street nurse Cathy Crowe, public relations analysts Claire LaRocca and Alen Sadeh, professor and media artist David Bouchard, and the staff at the Ryerson Image Arts Building. For the second iteration of the project, David Schnitmann replaced Robert Tu to provide additional technical support.

Sincerely,

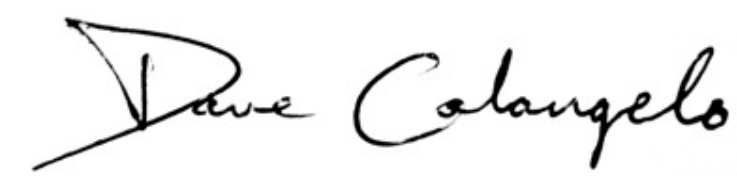

Dave Colangelo 
APPENDIX 3

Re: Letter Regarding Originality of Dissertation

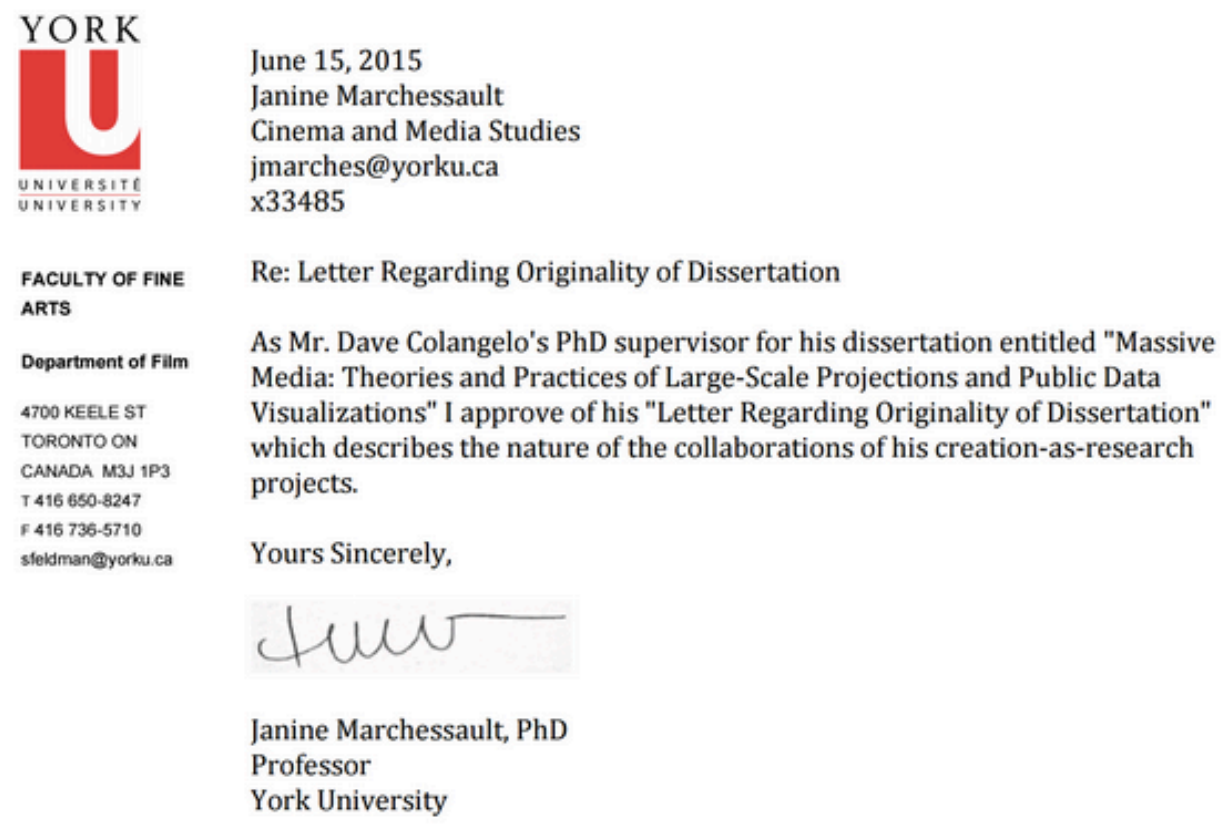




\section{BIBLIOGRAPHY}

Albera, Francois, and Maria Tortjada, eds. 2010. Cinema Beyond Film: Media Epistemology in the Modern Era. Amsterdam: Amsterdam University Press.

Antonelli, Paola. 2008. Design and the Elastic Mind. New York: Museum of Modern Art.

Ars Electronica. 2015. “About.” Ars Electronica. Accessed May 14, 2015. http://www.aec.at/about/en/.

ArtCOP21. 2015. "ArtCOP21." ArtCOP21 | An Exceptional City-wide Climate Festival in Paris. Accessed June 15, 2015. http://www.artcop21.com/.

Augé, Marc. 1995. Non-places: Introduction to An Anthropology of Supermodernity. London: Verso.

Bateman, Chris. 2013. "A Brief History of the Canada Life Weather Beacon.” blogTO, August 24. Accessed May 1, 2014. http://www.blogto.com/city/2013/08/a_brief_history_of_the_canada_life_weathe r_beacon/.

Baudry, Jean-Louis. 1975. "The Apparatus: Metapsychological Approaches to the Impression of Reality in the Cinema." In Narrative, Apparatus, Ideology: A Film Theory Reader, edited by Philip Rosen, 299-318. New York: Columbia University Press.

Barikin, Amelia, Nikos Papastergiadis, Audrey Yue, Scott McQuire, Ross Gibson, and Xin Gu. 2014. "Translating Gesture in a Transnational Public Sphere." Journal of Intercultural Studies. 35 (4): 349-365.

Benjamin, Walter. 1999. Illuminations. Trans. Harry Zorn. London: Pimlico.

Bennett, Susan. 2009. "The Peripatetic Audience." Canadian Theatre Review. 140 (Fall): 8 13.

Berman, Marshall. 2001. "Metamorphoses of Times Square." In Impossible Presence: Surface and Screen in the Photogenic Era, edited by Terry Smith, 39-69. Chicago: University of Chicago Press.

Berman, Marshall. 1982. All that is Solid Melts into Air: The Experience of Modernity. New York: Simon and Schuster.

Bishop, Claire. 2012. Artificial Hells: Participatory Art and the Politics of Spectatorship. London: Verso Books. 
Bishop, Claire. (ed.). 2006. Participation. London: Whitechapel.

Bishop, Claire. 2004. "Antagonism and Relational Aesthetics." October. 110: 51-79.

Björk. 2011. Mutual Core, London: Polydor.

Blatchford, Andy. 2008. "Massive Robert Lepage projection show tells 400 year story of Quebec City.” The Canadian Press, July 9, 2008.

Bradshaw, James. 2010. "Is Nuit Blanche a victim of its own success?" The Globe and Mail, October 1, 2010. Accessed May 1, 2015.

http://www.theglobeandmail.com/news/toronto/is-nuit-blanche-a-victim-of-its-ownsuccess/article4327778/.

Brougher, Kerry. 2008. "The Cinema Effect." In The Cinema Effect: Illusion, Reality, and the Moving Image. London: Giles.

Castells, Manuel. 2001, 2002. "Space of Flows, Space of Places: Materials for a Theory of Urbanism in the Information Age." In The City Reader, $3^{\text {rd }}$ edition, edited by Richard T. LeGates and Frederic Stout, 572-582. London: New York. 2003.

CBC. 2014. "In the Air, Tonight | Spark with Nora Young | CBC Radio." CBCnews, March, 2. Accessed May 16, 2014. http://www.cbc.ca/spark/blog/2014/03/02/responsive-art/.

Chapman, Owen, and Kim Sawchuk. 2012. "Research-Creation: Intervention, Analysis, and 'Family Resemblances'." Canadian Journal of Communication. 37: 5-26.

CNN. 2012. "CNN to Display Electoral Results Atop Empire State Building." CNN Press Room, November 6. Accessed May 1, 2014. http://cnnpressroom.blogs.cnn.com/2012/11/06/cnn-to-exclusively-displayelectoral-results-atop-the-empire-state-building/.

Colangelo, Dave, and Davila, Patricio. 2011. "Public Data Visualization: Dramatizing Architecture and Making Data Visible," conference proceedings, International Symposium on Electronic Art (ISEA), Istanbul, September 2011. Accessed May 1, 2015. http://isea2011.sabanciuniv.edu/paper/public-data-visualization-dramatizingarchitecture-and-making-data-visible

Collins, Glenn. 2007. "Lighting New York’s Tallest." New York Times, April 20, 2007. Accessed May 1, 2014. http://www.nytimes.com/video/nyregion/1194817113448/lighting-new-yorkstallest.html. 
Colomina, Beatriz. 2009. "Enclosed by Images: The Eames's Multiscreen Architecture." In Art of Projection, edited by Stan Douglas and Christopher Eamon, 36-56. Ostfildern, Germany: Harje Cantz.

Colosi, Nina. 2012. "Imagining Digital Cities." In Urban Media Cultures: (Re)Shaping the Public Space through Urban Screens and Media Architectures, edited by Susa Pop, Gernot Tscherteu, Ursula Stalder, and Mirjam Struppek, 48-53. Berlin: avedition.

Comolli, Jean-Louis. 1980. "Machines of the Visible.” In The Cinematic Apparatus, eds. Teresa De Lauretis and Stephen Heath, 121-142. New York: St. Martin's Press.

Connecting Cities. 2013a. "Networked City 2013." Connecting Cities. Accessed April 24, 2014. http://www.connectingcities.net/city-vision/networked-city-2013.

Connecting Cities. 2013b. "Technical Framework." Connecting Cities. Accessed April 24, 2014. http://www.aec.at/connectingcities/.

Connecting Cities. 2014a. "About.” Connecting Cities. Accessed April 24, 2014. http://www.connectingcities.net/about-connecting-cities.

Connecting Cities. 2014b. “TV.” Connecting Cities. Accessed April 24, 2014. http://www.connectingcities.net/tv.

Connolly, Maeve. 2013. "Artangel and the Changing Mediascape of Public Art." Journal of Curatorial Studies. 2 (2): 196-217. doi: 10.1386/jcs.2.2.196_1.

Crary, Jonathan. 1999. Suspensions of Perception: Attention, Spectacle and Modern Culture. Cambridge, MA: MIT Press.

Cubitt, Sean. 2007. "Precepts for Digital Artwork.” In Fluid Screens, Expanded Cinema, edited by Janine Marchessault and Susan Lord, 304-320. Toronto: University of Toronto Press.

Debord, Guy. 2009. Society of the Spectacle. Translated by D.N. Smith. New York: Zone Books.

Deleuze, Gilles. 1992. "Postscript on the Societies of Control." October. 59: 3-7.

Demers, Amélie. 2010. "L’Offre Nocturne de la ville de Québec: Vers un Tourisme de la Nuit." Rabaska. 8: 43-49.

De Souza e Silva, Adriana. 2006. "From Cyber to Hybrid." Space and Culture. 9 (3): 261 278. 
De Souza e Silva, Adriana. and Jordan Frith. 2012. Mobile Interfaces in Public Spaces. New York, NY: Routledge.

Doane, Mary Ann. 2009. "The Location of the Image: Cinematic Projection and Scale in Modernity." In The Art of Projection, edited by Stan Douglas and Christopher Eamon, 151-166. Stuttgart: Hatje Cantz.

Dubé, Philippe. 2012. “The Image Mill: A Sense of Place for a Museum of Images.” In Making Sense of Place, edited by Ian Covery, Gerard Corsane, and Peter Davis. Woodbridge, UK: The Boydell Press.

Ebsen, Tobias. 2013. "Material Screen: Intersections of Media, Art, and Architecture." PhD thesis. Faculty of Arts, Aarhus University, Denmark. Accessed April 24, 2014. http://tobiasebsen.dk/research/phd-dissertation/.

Empire State Building. 2014. "Historical Timeline." The Official Site of the Empire State Building. Accessed May 1, 2014.

http://www.esbnyc.com/esb_story_historical_timeline.asp.

EX MACHINA. 2014. "The Image Mill ${ }^{\mathrm{TM}}$.” Accessed July 3, 2014. http://lacaserne.net/index2.php/other_projects/the_image_mill/.

Fletcher, Harrell, and July, Miranda. 2002-09. “Learning to love you more.” Accessed December 8, 2015. http://www.learningtoloveyoumore.com/index.php.

Foster, Hal. 2010. "New Monumentality: Architecture and Public Space.” In The Real Perspecta 42, edited by M. Roman and T. Schori. Cambridge, MA: MIT Press.

Foucault, Michel. 1980. Power/Knowledge: Selected Interviews and Other Writings, 1972-1977. New York: Pantheon.

Frampton, Kenneth. 1983. Towards a Critical Regionalism: Six Points for an Architecture of Resistance. In Postmodern Culture, edited by Hal Foster, 16-30. London: Pluto Press.

Fuller, Matthew. 2005. "How This Becomes That." In Media Ecologies: Materialist Energies in Art and Technoculture, 85-107. Cambridge, MA: MIT Press.

Galloway, Alexander. 2012. The Interface Effect. Cambridge: Polity.

Garber, Megan. 2012. "The Empire State Building Becomes an Art Installation." The Atlantic, November 27, 2012. Accessed May 09, 2014. http://www.theatlantic.com/technology/archive/2012/11/the-empire-statebuilding-becomes-an-art-installation/265652/. 
Giedion, Sigfried. 1944. "The Need for a New Monumentality” In New Architecture and City Planning, edited by Paul Zucker, 549-568. New York: F. Hubner \& Co.

Giedion, Sigfried, Leger, Ferand, and Sert, Jose Luis. 1958. "Nine Points on Monumentality." In Architecture, You and Me, the Diary of a Development, edited by Sigfried Giedion, 48-51. Cambridge: Harvard UP.

Graham, Beryl and Sarah Cook. 2010. Rethinking Curating: Art after New Media. Cambridge, MA: The MIT Press.

Guattari, Felix. 2000. The Three Ecologies. Translated by Ian Pindar and Paul Sutton. London: The Anthalone Press.

Gunning, Tom. 2007. "To Scan a Ghost: The Ontology of Mediated Vision." Grey Room 26. (Winter): 94-127.

Gunning, Tom. 1990. "The Cinema of Attractions: Early Film, Its Spectator and the Avant-Garde." In Early Cinema: Space, Frame, Narrative, edited by Thomas Elsaesser with Adam Barker. London: British Film Institute.

Habermas, Jurgen. 1989. The Structural Transformation of the Public Sphere: An Inquiry Into a Category of Bourgeois Society. Cambridge, MA: MIT Press.

Habermas, Jurgen. 1974. "The Public Sphere: An Encyclopaedia Article (1964).” New German Critique. 3: 49-55.

Hansen, Mark B.N. 2006. Bodies in Code: Interfaces with Digital Media. New York: Routledge.

Hansen, Miriam Bratu. 1993. "Unstable Mixtures Dilated Spheres: Negt and Kluge's the Pubic Sphere and Experience, Twenty Years Later." Public Culture. 5: 179-212.

Hansen, Miriam Bratu. 1995. "America, Paris, the Alps: Kracauer (and Benjamin) on cinema and modernity." In Cinema and the invention of modern life, edited by L. Charney and V.R. Schwartz, 362-402. Berkeley, CA: University of California Press.

Harbord, Janet. 2007. The Evolution of Film: Rethinking Film Studies. Cambridge: Polity.

Hardt, Michael. 2009. "Production and Distribution of the Common: A Few Questions for the Artist." Open 16. Accessed April 24, 2014. http://www.skor.nl/_files/Files/OPEN16_P20-28.pdf.

Ibelings, Hans. 2002. Supermodernism: Architecture in the Age of Globalization. NAi Publishers: London. 
Kenner, Hugh. 1975. A Homemade World: The American Modernist Writers. New York: Morrow.

Keromnes, Chloe. 2015. "L'Arc de Triomphe s'illumine des mots 'Paris est Charlie' RTL.” RTL, January 9. http://www.rtl.fr/actu/societe-faits-divers/l-arc-de-triomphes-illumine-des-mots-paris-est-charlie-7776159636.

Kester, Grant. 2006. "Crowds and Connoisseurs: Art and the Public Sphere in America." In A Companion to Contemporary Art Since 1945, edited by Amelia Jones, 262-67. Oxford: Wiley-Blackwell.

Kracauer, Sigfried. 1965. Theory of Film; the Redemption of Physical Reality. London: Oxford University Press.

Krajewski, Piotr. 2005. From Monument to Market: Video Art and Public Space. Edited by Violetta Kutlubasis-Krajewska. Wroclaw, Poland: WRO Center for Media Art.

Krysa, Joasia. 2006. Curating Immateriality: The Work of the Curator in the Age of Network Systems. Brooklyn, NY: Autonomedia.

"La Ville De Quebec Confirme Que Le Moulin à Images en Sera à Sa Dernière Annee." 2013. La Presse Canadienne, April 1. http://ezproxy.lib.ryerson.ca/login?url=http://search.proquest.com/docview/1323 328011 ? accountid=13631.

Landry, Charles. 2008. The Creative City: A Toolkit for Urban Innovators. London: Earthscan.

Lefebvre, Henri. 1997. “The Monument.” In Rethinking Architecture: A Reader in Cultural Theory, 133-137. London: Routledge.

Lefebvre, Henri. 1991. The Production of Space. Oxford, UK: Blackwell.

Lepage, Robert. 2011. Le Moulin à images: L'événement phare du $400^{\circledR}$ anniversaire de la ville de Québec. DVD. Quebec: National Film Board, 2011.

Lessard, Bruno. 2009. "Site-Specific Screening and the Projection of Archives: Robert Lepage’s Le Moulin à images.” PUBLIC. 40: 70-82.

Lewis, Paul, Jon Swaine and Ben Jacobs. 2015. “This is not the justice we seek': sorrow in Baltimore as grief turns into riots.” The Guardian, April 28, 2015. Accessed May 8, 2015. http://www.theguardian.com/us-news/2015/apr/28/baltimore-riots-firesreaction-looting-protesters-hurt-community-unrest-just-beginning 
Lind, Maria. 2010. Selected Maria Lind Writing. Edited by B. K. Wood. Berlin: Sternberg Press.

Lynch, Kevin. 1960. The Image of the City. Cambridge, MA: The MIT Press.

Manovich, Lev. 2008. "Introduction to Info-Aesthetics.” Retrieved Dec 20, 2014, from http://manovich.net/content/04-projects/058-introduction-to-info-aesthetics/57article-2008.pdf

McCarthy, Anna. 2001. Ambient Television. Durham, NC: Duke University Press.

McKim, Joel. 2014. "Distributed Design: Media Technologies and the Architecture of Participation.” In DIY Citizenship: Critical Making and Social Media, edited by Megan Boler and Matt Ratto, 283-294. Cambridge, MA: MIT Press.

McKim, Joel. 2012. "Spectacular Infrastructure: The Mediatic Space of Montreal's 'Quartier des spectacles'.” PUBLIC. 45: 128-138.

McQuire, Scott. 2009. "Mobility, Cosmopolitanism and Public Space in the Media City." In Urban Screens Reader, edited by Scott McQuire, Meredith Martin, and Sabine Niederer, 45-64. Amsterdam: Institute of Network Cultures.

McQuire, Scott. 2008. The Media City: Media, Architecture, and Urban Space. London: Sage.

Media Architecture Institute. 2015. "About.” Media Architecture Institute. Accessed May 27, 2015. http://www.mediaarchitecture.org/about/

Moholy-Nagy, Laszlo. 1969. Painting, Photography, Film. London: Lund Humphries.

Mouffe, Chantal. 2007. "Artistic Agonism and Agonistic Spaces.” Art EF Research: A Journal of Ideas, Contexts and Method. 1(2): 1-5.

Mumford, Eric Paul. 2000. The CIAM Discourse on Urbanism, 1928-1960. Cambridge, MA: MIT Press.

Murphie, Andrew. 2004. "The World as Clock: The Network Society and Experimental Ecologies." Topia: Canadian Journal of Cultural Studies. 11: 117-138.

Nolen, Stephanie. 2014. "In Canada Day first, Rio's Christ the Redeemer statue lit up 'like your flag." The Globe and Mail, July 1, 2014.

Nora, Pierre. 1989. "Between Memory and History: Les Lieux de Mémoire." Representations. 26 (Spring): 7-24. 
Paik, Nam June. 1984. "Nam June Paik - Good Morning Mr Orwell (1984).” YouTube. Accessed April 24, 2014. https://www.youtube.com/watch?v=0oUdI-KFCyU.

Philips. 2012. "Press Information." The Official Site of the Empire State Building, May 9. Accessed May 1, 2014. http://www.esbnyc.com/documents/press_releases/2012_05_09_Philips_Press_Re lease.pdf.

Philips, Patricia C. 2003. "Creating Democracy: A Dialogue with Krzysztof Wodiczko." Art Journal. 62(4): 32-47.

Phillips, Patricia C. 1989. "Temporality and Public Art.” Art Journal. 48 (4): 331-35.

Quartier des spectacles. 2014. "McLarena.” McLaren Wall-to-Wall. Accessed July 5, 2014. http://www.mclarenmuramur.com/en/works/mclarena.

Rancière, Jacques. 2000. The Politics of Aesthetics. Translated by Gabriel Rockhill. London: Continuum.

Rodowick, David Norman. 2007. The Virtual Life of Film. Cambridge, MA: Harvard University Press.

Rokeby, David. 1995. "Transforming Mirrors: Subjectivity and control in interactive media," In Critical issues in electronic media, edited by Simon Penny, 133-158. Albany, NY: SUNY Press.

Rowlands, Michael, and Christopher Tilley. 2006. "Monuments and Memorials." In Handbook of Material Culture, edited by Christopher Tilley, Webb Keane, Susanne Küchler, Michael Rowlands and Patricia Spyer, 500-516. London: SAGE Publications Ltd.

Saidi, Habib. 2012. "Capital Cities as Open-air Museums: A Look at Québec City and Tunis" Current Issues in Tourism. 15: 1-2, 75-88.

Scott, Felicity D. 2003. "Involuntary Prisoners of Architecture.” October. 106 (Fall): 75-101.

Sellmer, Robert. 1946. "Douglas Leigh: The Man Whose Gadgets Brightened Up Broadway Now Turns to Main Street." Time, April 1, 47-51.

Shental, Andrey. 2013. "In the Junkyard of Wrecked Fictions." Mute. 14 May. Accessed July 4, 2014. http://www.metamute.org/editorial/articles/junkyard-wreckedfictions. 
Simmel, Georg. 1950. "The Metropolis and Mental Life." In The Sociology of Georg Simmel, translated by Kurt Wolff, 409-424. New York: Free Press.

Sloan, Johanne. 2006. "At Home on the Street: Public Art in Montreal and Toronto." In Urban Enigmas, edited by Johanne Sloan. Montreal and Kingston: McGill-Queen's University Press.

Smith, Hazel and R. T. Dean. 2009. Practice-led Research, Research-led Practice in the Creative Arts. Edinburgh: Edinburgh University Press.

Sokol, Zach. 2014. "Tweeting About \#Homelessness Tonight Will Change The Color Of This Building | The Creators Project." The Creators Project, February, 4. Accessed May 16, 2014. http://thecreatorsproject.vice.com/blog/tweeting-abouthomelessness-will-change-the-color-of-this-building.

Streaming Museum. 2013. "About." Streaming Museum. Accessed April 24, 2014. http://streamingmuseum.org/.

Tauranac, John. 1995. The Empire State Building: The Making of a Landmark. New York: Scribner.

Taylor, Diana. 2007. The Archive and the Repertoire: Performing Cultural Memory in the Americas. Durham, NC: Duke University Press.

Thain, Alanna. 2008. "Affective Commotion: Minding the Gap in Research-Creation." INFLeXions. 1: 1-12.

The Canadian Press. 2008. "Massive Robert Lepage Show Reflects Quebec City's Story." CBCnews. July 10, 2008. Accessed July 3, 2014. http://www.cbc.ca/news/canada/montreal/massive-robert-lepage-show-reflectsquebec-city-s-story-1.706088.

"The NEW Empire State Building Lights - Live Show with Alicia Keys." YouTube. 2012. November 26, 2012. Accessed May 12, 2014. https://www.youtube.com/watch?v=PKVzdqYSkks.

Thrift, Nigel. 2008. Non-representational Theory: Space/Politics/Affect. New York: Routledge.

Times Square Arts. 2014. "Midnight Moment Archive.” Times Square. Accessed August 5, 2014. http://www.timessquarenyc.org/times-squarearts/moment/archive/index.aspx\#.U-ZHMbsQA_E. 
Toft, Tanya. 2012. "Interview: Maurice Benayoun.” Streaming Museum. Accessed April 24, 2014. http://streamingmuseum.org/maurice-benayoun-occupies-the-financialdistrict-nyc/.

Toft, Tanya. 2013a. "Interview: Nina Colosi." Urban Media Aesthetics. Accessed April 24, 2014. http://urbanmediaaesthetics.org/\#?cat=11_interviews?post=1185_ninacolosi-founder-and-creative-director-of-streaming-museum.

Toft, Tanya. 2013b. "Interview: Susa Pop." Urban Media Aesthetics. Accessed April 24, 2014. http://urbanmediaaesthetics.org/\#?cat=11_interviews?post=1159_susa-popfounder-of-public-art-lab-and-director-of-connecting-cities.

Tuer, Dot. 1997. "Mirrors and Mimesis: An Examination of the Strategies of Image Appropriation and Repetition in the Work of Dara Birnbaum." n.paradoxa. 3: 4-16. Accessed January 10, 2014. http://www.ktpress.co.uk/pdf/nparadoxaissue3_DotTuer_4-16.pdf.

Turner, Fred. 2012. "The Family of Man and the Politics of Attention in Cold War America." Public Culture. 24(1): 55-84.

Turner, Fred. 2013. The Democratic Surround: Multimedia $\mathcal{E}$ America Liberalism from World War II to the Psychedelic Sixties. Chicago: The University of Chicago Press.

Tweedie, Steven. 2015. "Meerkat's CEO Describes What it was Like the Day Twitter Decided to Crush his App: 'The team went back to the office to get ready to dive in'.” Business Insider, May 6, 2015. Accessed May 8, 2015. http://www.businessinsider.com/meerkat-has-nearly-2-million-users-2015-5.

UNESCO. 2014. "Historic District of Old Québec." - UNESCO World Heritage Centre. Accessed July 03, 2014. http://whc.unesco.org/en/list/300.

Vande Moere, Andrew, and Hill, Dan. 2012. "Designing for the Situated and Public Visualization of Urban Data." Journal of Urban Technology. 19 (2): 25-46.

Vande Moere, Andrew, and Wouters, Niels. 2012. "The Role of Context in Media Architecture.” ACM Symposium on Pervasive Displays (PerDis'12), ACM. Article 12.

Verhoeff, Nanna. 2012. Mobile Screens: The Visual Regime of Navigation. Amsterdam: Amsterdam University Press.

Virilio, Paul. 1986. “The Overexposed City." L'espace critique (Paris: Christian Bourgeois, 1984); translated in Zone 1-2 (New York: Urzone, 1986), translated by Astrid Hustvedt: $14-31$. 
Wagner, Geraldine B. 2003. Thirteen Months to Go: The Creation of the Empire State Building. San Diego, CA: Thunder Bay Press.

Warner, Michael. 2005. Publics and Counterpublics. New York: Zone Books.

Wodiczko, Krzysztof. 2015. "The Inner Public” FIELD, 1. Accessed June 1, 2015. http://field-journal.com/issue-1/wodiczko.

Yin, Robert K. 2012. Applications of Case Study Research. Thousand Oaks, CA: SAGE.

Youngblood, Gene. 1970. Expanded Cinema. New York: Dutton. 